\title{
Spatial and Temporal
}

Dimensions for

Legal History

Research Experiences and Itineraries

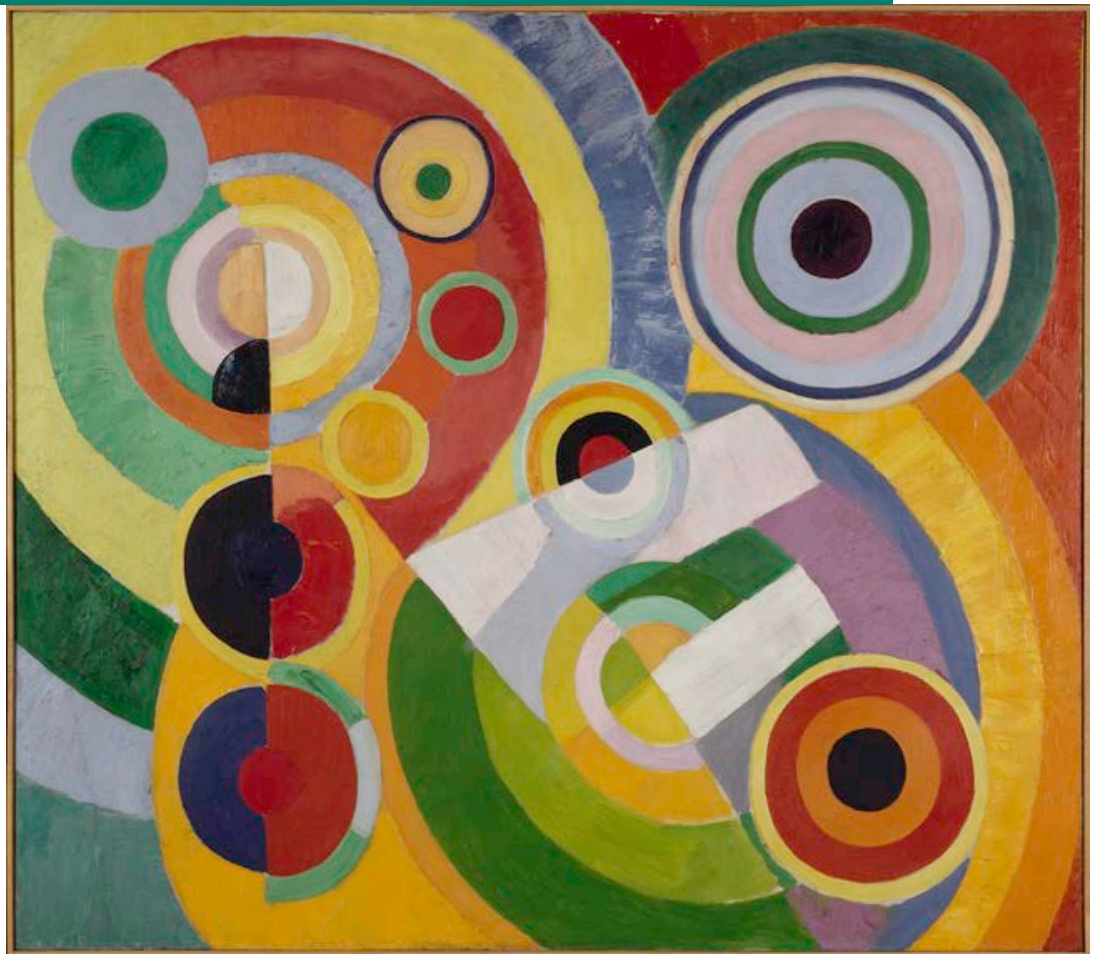



GLOBAL PERSPECTIVES
ON LEGAL HISTORY 


\title{
Global Perspectives on Legal History
}

\author{
A Max Planck Institute for European Legal History \\ Open Access Publication
}

http://global.rg.mpg.de

Series Editors: Thomas Duve, Stefan Vogenauer

Volume 6

Global Perspectives on Legal History is a book series edited and published by the Max Planck Institute for European Legal History, Frankfurt am Main, Germany.

As its title suggests, the series is designed to advance the scholarly research of legal historians worldwide who seek to transcend the established boundaries of national legal scholarship that typically sets the focus on a single, dominant modus of normativity and law. The series aims to privilege studies dedicated to reconstructing the historical evolution of normativity from a global perspective.

It includes monographs, editions of sources, and collaborative works. All titles in the series are available both as premium print-on-demand and in the open-access format. 
MASSIMO MECCARELLI

MARÍA JULIA SOLLA SASTRE (EDS.)

\section{Spatial and Temporal Dimensions for Legal History}

Research Experiences and Itineraries

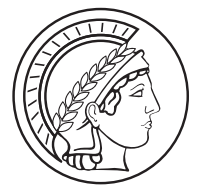

MAX PLANCK INSTITUTE

FOR EUROPEAN LEGAL HISTORY

2016 
ISBN 978-3-944773-05-6

eISBN 978-3-944773-15-5

ISSN 2196-9752

First published in 2016

Published by Max Planck Institute for European Legal History, Frankfurt am Main

Printed in Germany by epubli, Prinzessinnenstraße 20, 10969 Berlin

http://www.epubli.de

Max Planck Institute for European Legal History Open Access Publication

http://global.rg.mpg.de

Published under Creative Commons CC BY-NC-ND 3.0 DE

http://creativecommons.org/licenses/by-nc-nd/3.0/de

The Deutsche Nationalbibliothek lists this publication in the Deutsche Nationalbibliographie; detailed bibliographic data are available on the Internet at http://dnb.d-nb.de

Cover illustration:

Robert Delaunay, Rythme, Joie de vivre, 1930

Centre Pompidou - Musée national d'art moderne, Paris

(C) bpk Bildagentur für Kunst, Kultur und Geschichte, Berlin

Cover design by Elmar Lixenfeld, Frankfurt am Main

Recommended citation:

Meccarelli, Massimo, Solla Sastre, María Julia (eds.) (2016), Spatial and Temporal Dimensions for Legal History. Research Experiences and Itineraries, Global Perspectives on Legal History, Max Planck Institute for European Legal History Open Access Publication, Frankfurt am Main, http://dx.doi.org/10.12946/gplh6 


\section{Contents}

\section{Introduction}

3 | Massimo Meccarelli, María Julia Solla Sastre

Spatial and Temporal Dimensions for Legal History:

An Introduction

\section{Experiences}

\section{7 | Pietro Costa}

A 'Spatial Turn' for Legal History? A Tentative Assessment

63 | Javier Barrientos Grandon

Sobre el "Espacio" y el "Tiempo" y el "Estado de las Personas".

Una mirada desde la Historia del Derecho

101 | Alejandro Agüero

Local Law and Localization of Law. Hispanic Legal Tradition and Colonial Culture $\left(16^{\text {th }}-18^{\text {th }}\right.$ Centuries)

131 | Marta Lorente Sariñena

Uti possidetis, ita domini eritis. International Law and the Historiography of the Territory

\section{Itineraries}

175 | Paolo Cappellini

Carl Schmitt revisited. Ripensare il Concetto di 'Grande Spazio' (Großraum) in un Contesto Globale 


\section{5 | Laura Beck Varela}

The Diffusion of Law Books in Early Modern Europe:

A Methodological Approach

\section{Floriana Colao}

Per una Storia del Processo Penale «all'Italiana». «Astratte

Modellistiche» e «Abitudini Profondamente Radicate»

279 | Giacomo Pace Gravina

Beyond the Lighthouse. Sicily and the 'Sicilies':

Institutional Readings of a Borderland

\section{9 | Contributors}




\section{Introduction}





\author{
Massimo Meccarelli \\ María Julia Solla Sastre
}

\title{
Spatial and Temporal Dimensions for Legal History: An Introduction
}

\author{
“Zum Raum wird hier die Zeit” (Parsifal, erster Aufzug)
}

\section{Time and Space: A Historiographic Approach}

Where is legal history heading today? This is a concern that is not exclusive to those of us who encouraged the dialogue that gave rise to this volume. ${ }^{1}$ Indeed, in recent times broad sectors within the academic community have experienced a sense of having gone adrift with regard to the history that, in general terms, has ceased to meet a very concrete - and prolific - aim in the construction of national entities, and is in need of new challenges to endow it with meaning as a discipline.

Assuredly, legal history's bearings are an issue directly related to the purpose we want it to achieve, and therefore, the specific aim toward which we wish to guide it. The perception over the last few years, however, is that we are bereft of a firm course or major themes, aims or perspectives.

Despite this uncertainty, however, two facts are clearly discernible. The first is that we must continue to reflect deeply and persistently on the problems and challenges posed in a global world to the social and legal sciences

1 The essays that have shaped this book are the result of the proposals and reflections shared at the international seminar Ambiti geografici e dimensioni temporali nella storia del diritto: esperienze e percorsi di ricerca, held at the Scuola Superiore Giacomo Leopardi, Universita di Macerata, on $20^{\text {th }}$ and $21^{\text {th }}$ June 2013. This event took place within the framework of the international agreement between Universidad Autónoma de Madrid and Università degli Studi di Macerata. The contributions of the members of the former institution (A. Agüero, J. Barrientos, L. Beck, M. Lorente and J. Solla) have been carried out within the research project of the Spanish Ministry of Science and Innovation with reference DER2014-56291-C3-1-P. We extend our thanks to Thomas Duve for his proposal to publish this volume as part of this collection, and to Ludovica Bosica for her diligent editing. 
in the West. ${ }^{2}$ In fact, serious reflection is being fomented by researchers and scientific groups of considerable renown that is of direct interest to legal historians.

To set an example, the celebration of the fortieth anniversary of the Quaderni forentini per la storia del pensiero giuridico moderno was dedicated to examining the state and the course on which legal historiography is set. ${ }^{3}$ Moreover, the constant initiatives taken by the Max-Planck-Institut für europäische Rechtsgeschichte have been opening up for some time now new horizons for a European legal history from a global perspective. ${ }^{4}$ Even the American Journal of Legal History has inaugurated its new stage with a re-launching issue devoted to "The Future of Legal History". 5 From a more general viewpoint, the debates in the Anglo-American world are likewise crystallizing in suggestive proposals and mechanisms for the recovery of the role of history to illustrate contemporary problems and to envision balanced democratic governance and public policies for the long haul. ${ }^{6}$

All these contributions within the framework of a large-scale mobilization serve to exemplify the second aspect we consider to be beyond question: there can be no doubt that this reflection must be collective. The idea of collectiveness surpasses that of the sum of individual efforts, to become inserted in the sphere of major lines of cooperative research. Consequently, the third aspect that emerges clearly is that we need to reconsider that which links us through our specificity, as two indispensable elements in the historian's trade: time and space.

Indeed, within this difficult debate over the common destination of this discipline, we have considered it necessary to turn our regard to the spatialtemporal conjunction that, aside from various ideological rapprochements and methodological differences, is indefectibly shared in the legal reflection on the historicity of law and the need to historify its use through other disciplines.

These, in a word, are the elements underpinning the reflection offered herein on this state of affairs.

2 Duve (2014b); RenN (2014); McCarthy (2014).

3 Sordi (2013). Regarding the MPIeR, a good example is found in Duve (2014a), and in the series said volume opens: Global Perspectives on Legal History.

4 Duve (2012); Duve (2014b).

5 http://ajlh.oxfordjournals.org/content/56/1.

6 Guldi/Armitage (2014); Armitage/Guldi (2015). 
Nowadays, no-one could question the contextual character of space and time through history. Or that the manner in which legal spaces and times and space and time in law - have been conceived has been inseparably linked to the historical contexts in which those times and spaces were imagined or perceived should be beyond all discussion for historians. Similarly, historians' understanding of time and space in their context has conditioned, limited or opened up new possibilities for the comprehension of law, and may even be found in the groundings of the definition of law itself.

Against this background, our proposal consists of rethinking key confluences that lie at the base of all iushistorical constructs, in order to provide coordinates for this collective reflection. We do not, however, aim to draw up abstract considerations on methodology, but to rely on concrete researches from which stems a reflection on the conjunction of space and time, as well as on the reconstruction of certain lines of research that hold a spatiotemporal component. The volume, therefore, offers this reflection articulated in two major blocs: "Experiences" and "Itineraries", comprising essays by Pietro Costa, Javier Barrientos, Alejandro Agüero, Marta Lorente, Paolo Cappellini, Laura Beck, Floriana Colao and Giacomo Pace.

From these different and enriching contributions we draw a number of conclusions that we shall endeavour to present below, given that in effect, they all reflect on the - declared or undeclared - use made throughout history of space and time as the essential tools of legal historians and legal histories.

\subsection{Uses of spatiotemporal coordinates}

For Michel Foucault "space itself has a history in Western experience and it is not possible to disregard the fatal intersection of time with space". ${ }^{7}$ Despite the fatality of this encounter, space-time dynamics have not received particular consideration as a theme in itself either in legal historiography or in the social sciences in general, which, as pointed out by Pietro Costa, have not only remained over an extended period of time "blind" to this conjunction that constitutes legal phenomena, but have also, even when addressing the historical study of international litigations on limits and borders, turned 
their backs on the spatiotemporal historicity of the very origins of conflicts (Lorente).

Pietro Costa invites us, therefore, to take awareness of spatiotemporality as a dimensional conjunction that is not only a presupposition in historical research, but that also generates a use that is susceptible of being studied in its own right. But Marta Lorente also reminds us that its reuse from new disciplinary viewpoints must follow certain guidelines and be conducted with caution.

One of these is to abandon certain uses that fulfilled a historical function as the foundational constituent of certain long-term units of power, such as the construction of the national unitary State, ${ }^{\mathbf{8}}$ but that today must be dismantled: it is necessary to surpass, if not already surpassed by the imperative reality of transnational law, ${ }^{9}$ the idea of State as an institutional player that is the protagonist taking full stage in legal and constitutional production and application (Agüero, Lorente). At the same time, however, it is equally necessary to detect, in the first place, and then to dismantle, the Eurocentric positioning that has served, in a manner similar to national histories, to define, explain and to justify Europe self-referentially, but that has not served to understand it unless inscribed within a global perspective of episodes, flows, players and forces of the widest diversity. ${ }^{\mathbf{1 0}}$

Thus, generally speaking, it appears to be absolutely necessary to abandon monistic conceptions of space and time which, on the one hand, restrict political, social and normative pluralism that is difficult to reconcile with rigid, unitary structures and, on the other hand, consider unity to be unavoidably paired with uniformity (Agüero, Costa). However, the ease with which the limitations to certain past instrumentalizations of spatiotemporality can be detected contrasts with the difficulty of projecting new uses for the future and progressing toward new levels of analysis in line with an aim that is still being defined.

\subsection{Paths of approach to spatiotemporality}

With these premises, the authors in this book offer several proposals for addressing the spatiotemporal from the perspective of legal history. The point

8 Raffestin (2013).

9 Vid. Duve (2014b).

10 Duve (2014b); ModéEr (2014). 
of departure is identified by Pietro Costa from among a confusion of spatiotemporal elements: it is necessary to study the interaction between space and time and, consequently, it is necessary to discern which are the instruments that connect not only diverse spaces and times, but also spaces and times to each other within diverse spaces and times. It is, in fact, a matter of detecting and studying spatiotemporality as a legal phenomenon (Agüero, Barrientos, Beck, Lorente).

A direct consequence of the foregoing is the need to address this phenomenon from the perspective of interdisciplinarity. In effect, given that time and space are two concepts that cut across any social discipline, grasping the density of spatiotemporality is inconceivable without exercising a joint vision of those dimensions. Thus, inescapable dependencies arise, such as that anticipated by Pietro Costa between geography and legal history; likewise, notable uses are also found, namely sociology or anthropology in concepts such as localization (Agüero); or the interdependence of one history, that of the book, with another, the history of the sources of law (Beck). We also find clear interdependences within the legal disciplines themselves, such as between history and international law (Lorente).

This interdisciplinary approach, especially within the framework of legal disciplines, precisely reveals the artificial nature of a classification by subjects that generates added complexity on the historical front and which also needs to be dismantled in order to comprehend the structure of the origins of space and time in legal phenomena. Interdisciplinarity cannot imply unawareness of the autonomy of 'juridicality' and its potential for social and cultural creation, but nevertheless entails historicity. In this sense, perhaps, the exercise of seeking the origins may cause the fragmentary modern notion of multidisciplinarity in the object of this study to come apart. Effectively, returning to the roots may uncover how, at certain foundational moments, juridicality gradually absorbed - in the absence of contradictions or barriers of any kind - concepts that, today, we would place in other fields of knowledge, to build the very essence of juridicity from given phenomena. This process can be appreciated fully, for instance, with regard to a person's status, which is progressively being incorporated in legal discourse (Barrientos). ${ }^{11}$

11 From this perspective, see also Cappeldini (2010) 49-109. 
Indeed, another approach to spatiotemporality is a return to its origins, which in turn implies two inherent and unavoidable complications. The first of these difficulties is, precisely - as commonly occurs in legal history - pinpointing that original moment and thereby unmasking the limits that exist on the threshold to the determination of law, ${ }^{\mathbf{1 2}}$ to which we shall return later. All the above implies having understood models and paradigms, having unravelled elements of change and, especially, having scrutinised the spatiotemporal elements separating continuity from discontinuity (Lorente). Hence, a quest for the origins involves a particular and attentive examination of times and spaces that in fact cause a break, not always easy to detect in an apparent continuum.

The uti possidetis in the paper by Marta Lorente is a prime example of the need to dismantle a myth with regard to origins, having created, with the instruments of international law, the belief in a path of continuity from the categories of comprehension in a jurisdictional world and those of a world of States, heedless of their radical discontinuity. Another major difficulty in detecting the origins is found in the dynamics of permanence and continuity: this is the case, as set forth by Floriana Colao, of models that have survived through time. What is time in the case of these models? Do their readings of continuity change with each different space and time? Do they really remain just as they were conceived and understood, establishing a time of their own that surpasses other measures of time, thus becoming a space of time within others?

Yet a further difficulty, brought to our attention repeatedly by Alejandro Agüero, is that of singling out the appropriate analytical instruments to address said origins, once they have been located: we lack the elasticity of past concepts because we stem from a scenario - that of State law - that refused to imagine the world in a composite, heterogeneous manner, which it explained exclusively from a monolithic view comprising a number of subjects, namely the States, capable of generating units for the measurement of time and space that were highly homogeneous and comprehensible among said subjects.

However, we are currently immersed in a legal world in which statehood has become diluted in an ocean of non-State norms, and legal history is 
called upon to contribute to building new legal categories to explain this reality. Precisely this exercise in envisioning new scenarios and new chronologies obliges us to renew our awareness of the malleability of the realities contained in past concepts, however foreign they may seem to our present world, and consequently, to seek new concepts that help us to explain and accommodate with the greatest degree of detail as possible processes that, as in the case of the formation, interpretation or enforcement of laws, cannot be resolved in a linear or unidirectional manner in every legal situation (Agüero). It also invites us to find new viewpoints to rethink abandoned concepts, such as geopolitics (Cappellini), which despite having been important in their time and being brought to a close with the space and time to which they belonged, can be turned to afresh in the quest for new channels for naming complex realities that have exceeded the reality that brought them to a close.

\subsection{Dynamic perspective vs. static perspective}

If there is any clear notion to be drawn from the contributions making up this volume, this is that there is no true correspondence between space and staticity. Furthermore, it would be extremely reductive to state that space corresponds to a place, thus highlighting a static component that is not essential to space. A space charged with chronic dynamism, to take the case in point, is that which exists between those reading from the sources and the sources themselves (and of which the essay by Javier Barrientos ensures we retain awareness); a space that, in turn, as illustrated by Floriana Colao, is impregnated with a dynamic temporality, insofar as productions from times in the past are interwoven with readings from present times that either create history by consolidating this past-present décalage, or update history by smoothing over the potential breaks in continuity.

The dynamism of times and spaces is manifested in two ways: on the one hand, in the multiplicity of their confines. The players, according to their own interests, contemplate different dimensions in the spaces and diverse uses of time, which by no means necessarily correspond to political measures and confines, such as the commercial vision of the world held by publishers or censors when disseminating (or preventing the dissemination of) works (Beck); on the other hand, in the dynamism of those instruments that serve to create spaces and certain comprehensions of time and space. A useful 
example is given precisely in the essay by Laura Beck, on the subject of the "book" as a support, as an instrument connecting past, present and future spaces and times, making it possible to accomplish the unthinkable interrelationship between seemingly unconnected places and times. Further, it possesses the potential to transmit approaches that, in turn, lead to the construction of legal spaces: such is the case of ius patrium which began to populate the legal literature of the eighteenth century.

In this sense, so are the devices giving rise to spatiotemporalities dynamic in themselves, contributing to establish the logic of dynamism in the heart of cultural communities. ${ }^{\mathbf{1 3}}$ We refer to the various processes of interpretation and adaptation, transferences, etc. suffered by regulatory systems in relation with new spaces or different epochs (Agüero, Colao, Lorente). Likewise, the different instruments, in their movement and circulation, demonstrate the porosity, permeability and relativity of the borders established according to other parameters; or that this is true insofar as a concrete dimension is taken into account, such as politics, but ceases to be the case when different perspectives are taken, such as those of a cultural nature (Barrientos, Beck). ${ }^{\mathbf{1 4}}$ Similarly, this process shows up the supporting framework in the construction of actual spatial and temporal borders, the result of their reformulation through that same time and space.

Another dimension of dynamism are the processes of adaptation and modulation of the law into different spaces and times. There is a need to study law-modelling processes, from the awareness that the issue is not only that the law, which may be deemed pre-existent, should adapt to the conditions within a given location, but that the law should be justly formulated and materialised at the moment of being imported from a different space or another time and be properly channelled toward its specific formulation and materialisation for a given community (Agüero). Thus, not only does the time and space of a norm become updated with each new reformulation and embodied in a community but, at the same time, in the same exercise, the act of spatialisation becomes an intrinsic quality of the norm, ${ }^{15}$ as we shall see in the following section.

13 Turner (2008); Elias (1989).

14 Also Solla (2015b).

15 Meccarelli / Palchetti (2015b) 13-15. 
Envisioning new spaces or new conceptualisations of space, therefore, leads to thinking of a new chronology, while thinking of times and spaces differently allows us to alter the scale of representation and envisage new objects of study. ${ }^{16}$ Indeed, having removed the imperative nature of our spatiotemporal categories and become immersed in spatiotemporal dynamism, not only does the historian's regard detect new perspectives and dimensions in foregone themes, but new readings emerging from legal history: the historical component of legal history, although a privileged example (Lorente), is also an invitation to discover new areas for study in which the iushistorical perspective will shed much light on the artificiality of the origins of many subjects whose time and space has been determined (Cappellini). In short, an awareness of the dynamism of space and time (and the awareness the every space and time builds its own understanding of spaces and times, as exemplified by Giacomo Pace) makes it possible for us to see not only new perspectives on seemingly consolidated matters (Cappellini, Costa, Lorente), but also new fields of study in relation to legal history.

\section{Space and Law: Some Interpretative Categories}

To study the conjunction of the spatiotemporal we need to pay attention to these two elements with a certain degree of independence.

Let us begin with the spatial variable. The works published here apply a range of different treatments. In this section, we shall relate these to a number of interpretative devices of a more general nature. Mainly we aim to differentiate between two levels: space in a reconstructive sense, and space in a constitutive sense. ${ }^{\mathbf{1 7}}$

From the first of these points of view, perhaps the more recurrent in historiography, the problem posed by space in relation to the legal dimension operates instrumentally as an external factor that must be taken into account in order to refine the analytical focus. In line with this, it is the legal problem that determines the space of reference; hence, space has a reconstructive function.

16 Guldi / Armitage (2014); Armitage/Guldi (2015).

17 See also Meccarelli (2015). 
However, as confirmed in the research collected in this volume, it is also possible to consider the significance of the spatial dimension from within the legal dimension: that is to say, space as an implicit problem from the moment a legal question is formed. Here, space becomes a factor to the problem to be analysed, rather than being proposed as an analytical instrument. Thus, space assumes a value that is constitutive of the legal problem being addressed.

We shall now focus on this second interpretation, keeping an illustration of the first in mind throughout the following pages.

\subsection{Space as a determining factor of law}

Space, therefore, is an element that determines the legal object under observation; it is an internal element that constitutes our problem.

We could state, in agreement with Pietro Costa, that this valence becomes apparent on a bi-dimensional level: that of lived space and imagined space. This means that space, on the one hand, constitutes an «intrinsic component of social dynamics» and, on the other hand, represents a vision made explicit by society, as a moment's self-representation. Both of these horizons of spatiality contribute to the constitutional act of experience.

In the paper by Marta Lorente we also detect a use of space in constitutive key. Understanding the legal meaning of the principle of uti possidetis is possible as soon as we consider the perception of space inherited from the extended experience of the Monarquía católica. Such a space, necessarily understood in conjunction with the «jurisdictional and corporative nature of the political order» ${ }^{\mathbf{1 8}}$ - i. e., as an 'open' space that aggregates political powers and social bodies in a network of reciprocal relations of dependence and autonomy - constitutes a factor that justifies and explains the emergence of uti possidetis. Its meaning, therefore, is conditioned by the original premise, including when it is employed in the construction of intentionally closed spaces such as national or State spaces.

From our viewpoint, we can add that precisely by assuming this spatial variable in a constitutive sense we are able to appreciate this profile, and to 
restore to a principle such as uti possidetis its due performativity with regard to political options and programs.

The essay by Javier Barrientos, in turn, illustrates the theme of subjective status, in which space models the legal problem. Over the centuries from the modern age up to codification, space took on an increasingly prominent role in the definition of status: this was a process of spatialisation that 'degraded' the status in qualitas, thus allowing, even in an egalitarian context with a single and indeterminate subject, different spheres of individual freedom within a society to be reproduced.

The spatial factor, another example of which can be found in the contribution by Giacomo Pace dedicated to the territorial complexity of the Kingdom of the Two Sicilies, likewise has a central value in the definition of legalconstitutional configurations «beyond the lighthouse». Further examples could easily be found in this volume, but those mentioned thus far should suffice to highlight at least two heuristic paths found when considering space in a constitutive sense.

These planes can be inverted: then, (pre-)comprehension of space operates as an assumption enabling the legal problem to be individualised (as opposed to the situation in the case of reconstructive space, where the legal problem determines the space of reference).

A further, equally relevant analytical capability is likewise gained for the critical appraisal of the performativity of legal figures produced by experience. In effect, it is less a question of drafting the history of their emergence, but rather of their limits. The distinct perspective we believe can be discerned here consists in appreciating the sustainability of juridical concepts and legal figures produced through time. History, therefore, should be written attentive to the pre-comprehension profiles underpinning the conceptualization of ideas and to the pre-conditions determining concrete legal configurations. ${ }^{19}$

Not wishing, however, to establish bonds between these approximations to a necessarily shared programme we can state that, generally speaking, the use of time in constitutive key allows a degree of subsequent analysis that is enriching to the field of action of legal history and the range of questions (historical and theoretical) to which legal history can provide answers. 
This is how, to return to the point mentioned earlier, our discipline can be located in thematic fields with an effective interdisciplinary potential. All this - without lessening their identifying profiles - raises legal history's capacity for dialogue with other legal sciences (which, in view of current problems, pose questions regarding the appropriateness of the instruments used to address them) and social sciences (interested in incorporating the legal dimension to their own analytical grounding).

\subsection{Space as a field of action for law}

In this volume we also detect a further use of space in constitutive key, that we feel merits brief individual attention. This use emerges when we consider space as a field for the occurrence of law. Legal historiography has recently confirmed the significance of this perspective. ${ }^{20}$ Our volume contains, in particular, the pages by Alejandro Agüero that suggest this line of development. This author, in fact, considers the phenomenon from the standpoint of the dynamics of localization of law, viewed as a «constant process of interpretation aimed to adjust the rules to the precise conditions of time and place».

The scenario presented under this interpretative premise allows us to analyse an articulate legal space in which the spatiotemporal flow of law is characterised by a "complex interaction of factors that frame the local determination of law». This explains a process that refers to how an interpretation that 'locates' an intrinsically flexible law closes a cycle of perfection and materialisation of law, forming part of this self-same 'formulation' of law.

Here, the dynamism of law does not describe the movement of an object that is predefined and unchanging in time and becomes dislocated in space. To the contrary, it is seen as a prime environment in which to observe law as a dynamic object; an object that, despite having an identifiable provenance, adopts different characteristics as it negotiates spatial - or more aptly, spatiotemporal - movement.

Taking this approach to space in endeavouring to understand the flow of law is undoubtedly of specific importance to the study of legal structure in

20 See, for example, Clavero (2012); Hespanha (2013).

$14 \quad$ Massimo Meccarelli, María Julia Solla Sastre 
the Iberoamerican space (before and after the Monarquía católica); ${ }^{\mathbf{2 1}}$ nevertheless, we believe that this analytic approach can be applied with a wider scope, for example for the European perspective. In addition, it is applicable to different aspects of this complex phenomenon, covering rules and systems as well as doctrines or legal cultures.

It is, therefore, possible to make a general reflection on the opportunity of examining the dynamics of the flow of law in the light, shall we say, of spatialisation. As described above, by using this term we are underscoring the permanent tendency of law (understood in a broad sense) to take up a position in space, and to adhere to space. We wish thereby to highlight a process that has a bearing on the contents and configurations assumed in the legal dimension, a process that coexists with the moment of its manifestation. $^{22}$

It may be useful to mention some methodological implications that we believe derive from this constructive approach to the object under study.

We have recalled earlier that it was precisely Alejandro Agüero who drew our attention to the need to acquire the appropriate conceptual framework to interpret the relationship between space and law. In effect, there seems to be an imperative to surpass certain traditional modes of analysis (such as 'importation', 'reception', 'transplantation', 'acculturation'...) for referring to the spatiotemporal flow of law. ${ }^{23}$

For our perspective, it is inefficient and in certain aspects misleading to use a vocabulary that, used to describe the process of transmitting law from one place to another, emphasises the pre-existence of law and overlooks the aspect of its displacement in space. Instead, we must acquire an epistemic constellation of reference with which to capture the original meaning of the interconnection between space and law, making use for instance of the semantic potential of such terms as 'translation', 'hybridisation', 'appropriation', 'acculturation', etcetera.

To address spatialisation experiences and phenomena it is also necessary to concentrate on the cultural patterns acting as reactants in this process.

21 Lorente/ Portillo (2012). For later confirmations in this sense, please refer to Solla (2015b).

22 To this purpose, the idea of global entanglements proposed by ZIMMERManN (2014) is convincing. On spatialisation, see Meccarelli / Palchetti (2015b).

23 Duve (2014b); Meccarelli / Palchetti (2015b). 
These elements assume the role of instruments that facilitate the historification of singular legal experiences. The analysis at this point can be taken to considerable detail. Agüero, for example, regarding the foundations of the Hispanic Monarchy's dynamics of localization, individualises factors such as «particularism», «casuism», «normative factualism», or «hermeneutic frame linked to convenientia rerum transcendental principles of justice».

Finally, it seems appropriate to consider the possible feedback effects of the movement of law. Effectively, within its space of occurrence, the flow of law is no longer represented as a mono-directional phenomenon, as claimed in some recent works. ${ }^{24}$

This is not the occasion to develop such a reflection in depth. We have considered it of use, however, to at least mention these methodological implications in order to highlight the opening offered by an understanding of space as a theatre for the movement of law.

\subsection{Scales of spatiality}

The volume, despite opening a window to opportunities for analysis through the understanding of space in constitutive key, nevertheless also offers clues to re-considering the value of space in a reconstructive sense.

Of particular importance is the contribution relating to the criteria for determining the spatial scale of reference for the object of historical-legal analysis. In effect, this poses a crucial problem from the moment we attempt to use space as an instrument of iushistorical discourse. It is not our intention to draw up herein a closed list, but we can try to extract some of the spatial scale types used in the volume.

Space as place: this indicates considering the place in which to determine the space that is relevant to studying the legal problem. Space, in this case, far from being a Newtonian aprioristic conception of 'homogeneous space', is viewed as part of 'a multiplicity of different places', each with its own 'idiomatic and irreplaceable features' (Costa). This facilitates the development of spacing history in which the 'development of social phenomena is possible insofar as they "have place", are "located" somewhere' (Costa). This is not, therefore, a localist redeployment having lost all interest in (and any capacity to consider) major historical phenomena. To the contrary, such a

$24 \operatorname{Costa~(2004/2005);~Solla~(2015a)~and~(2015b);~Vitucci~(2015).~}$

16 Massimo Meccarelli, María Julia Solla Sastre 
'spacing legal history' enables the simultaneous consideration of several dimensions: it employs place as the key to interpreting the interconnections between the particular and the general dimension of law (Agüero, Costa). ${ }^{25}$ This also leads to greater awareness of legal history in relation to the dynamics of legal pluralism and multi-normativity. ${ }^{26}$

Internal spaces: the spatial dimension is also measurable in a diverse sense, that we can refer to as internal spaces. This spatial scale is distinct from local space, which operates in a context of complex and, to an extent, open legal spatialities. In the case of internal spaces, however, configurations involving closed legal spaces are considered, such as that represented by the State form (a form of legal spatiality characterized also by the formal determination of the territory on which it stands). This is the case of the Kingdom of the Two Sicilies discussed by Giacomo Pace.

An aspect that we find noteworthy is that no single internal spatiality exists within the territorial scope of the State. In fact, the essay by Pace shows, for example, the presence of multiple internal confines, and gives evidence of how multi-confinity is a relevant factor to defining the perspective on State space that has characterized those regions. The use of a scale of internal spaces has certain repercussions, owing to the fact that it places in the foreground players, institutional realities, or legal dimensions that would not stand out were they to remain within the formal pattern of articulating the State. For this reason we consider that careful analysis of internal spaces may be useful to offer a deeper perspective of the legal valence of the form of the State as historical experience.

The idea of internal space also appears to be useful for interpreting closed legal spaces. In these cases, the issue is to explain the genesis of certain closed spatial realities or, if you will, the process of transforming legal realities founded on the dialectic between "local" and "universal" in closed and self-sufficient legal realities. The problem of uti possidetis, as presented within the appropriate historical-legal coordinates by Marta Lorente, indeed entails special significance, as mentioned, in understanding the peculiarities accompanying Latin American State formations. ${ }^{27}$

25 This approach also in Clavero (2012); Hespanha (2013).

26 Respecting pluralism and multi-normativity as a field in legal history, see recent works by Duve (2014b).

27 Vid. for this purpose Lorente (2010); Lorente/ Portillo (2012). 
Extensive spaces: another spatial dimension relevant to historical-legal research is extensive spaces. ${ }^{28}$ We are referring to a comprehensive scale of spatiality that allows us to observe the interactions and interconnections among diverse legal phenomena. The legal historian's attention may turn to extensive spaces in different ways.

A parallel conception of spatiality can be addressed, such as that of great spaces which emerges «in tune with a 'Newtonian' view of space», discussed by Pietro Costa. From an analytical standpoint, this means considering, together with the closed spatiality of the Nation-State, an additional dimension, intertwined with the former, giving rise to a perspective of the experience of legal spatiality. In this manner it is possible to comprehend how the construction of international law in the nineteenth century has allowed the field of action of the Nation-State to be guided toward a «differentiated and heterogeneous spatiality» and has enabled «different combinations between space and politics»; this also holds for other phenomena pointed to by Costa that are supported by the premise of extensive spaces, such as colonial occupation, the emergence of the category of human rights or, in like manner, the reunion of State doctrines with geopolitics.

The latter is again subjected to analysis in the dense essay by Paolo Cappellini; in this case, recourse to an extensive spatial scale highlights its added analytical value. Recovering the problem of great spaces (considered from the Schmittian viewpoint, but also according to the proposal implicit in the Monroe doctrine) becomes, in fact, an opportunity not only for narrating a parabola of the modern State, but also to explicitly examine the perspectives of meaning in the international legal order (between the possibility of a unipolar global order and the pluriversum of great spaces). The idea of extensive spaces may be applicable beyond the closed spaces of the Nation-State, as suggested in the researches by Marta Lorente and Alejandro Agüero who employ this notion in their reasoning on the determination of legal spaces in a condition we could define as pre-State (Lorente) or local (Agüero).

28 For an updated reflection on the implications for legal history see Duve (2012) and (2014b). The problem is discussed in general also from the point of view of the historical sciences; for a historiographical treatment, see, recently, Armitage / Guldi (2015), Guldi / Armitage (2014). 


\section{Adscriptive Temporal Dimensions and Legal History}

The problem of space, as confirmed in these pages, is assuredly a major focus of attention from the moment we contemplate the issue of spatiotemporality. It is space, going back to Michel Foucault, that causes «the anxiety of our era», while time is merely «one of the various distributive operations that are possible for the elements that are spread out in space». ${ }^{29}$

We find this statement persuasive but, at the same time, find it necessary to remark that in considering time, we are obliged to appreciate the complexity of the temporal dimension of historical phenomena, most especially those of a legal-historical nature. ${ }^{30}$ Reviewing the articles composing this volume, the trend mentioned earlier regarding the relationship between space and law can be seen applied to the relationship between legal problem and time.

In this case the time factor appears to operate as the adscriptive rather than descriptive moment of a legal problem. On this point, we wish to underscore that our authors, despite dealing with very different subject matter, consider the time element as an inner feature of the legal problem in hand, that has a bearing on its nature, scope and development dynamics.

To give an example, this approach may be applicable to the principle of uti possidetis (Lorente), in which past time (as well as its rhetorical value in legitimating new legal forms, in relation to new political structures) determines the appropriate perspective to comprehend the construction of the State. Similarly, the time factor is seen as one of the aspects upon which doctrine acts to offer new meanings for the legal status (Barrientos) in a context that favours spaces for individual liberties and a unitary type of legal subject. The «creation and diffusion of legal knowledge», in turn, implies a temporal dimension (as well as spatial) as its determining moment (Beck).

Many more examples can be found, as it is precisely this persistence in time of the ritual forms in criminal justice («without the political time» in which it is applied) that explains, beyond abstract models of legal theory, the nature of Italian criminal procedures in the nineteenth and twentieth centuries (Colao). The time factor is an integrating element in the localization process of law (Agüero), as law is often articulated through legal instruments

29 Foucault (1986) 23.

30 See, in this respect, Paixão (2013); for a more general treatment, see also Hartog (2015); Armitage/Guldi (2015); Guldi / Armitage (2014); Marramao (2008); Elias (1989). 
created in a different temporal (as well as spatial) context. Finally, to round off this brief list of examples, an adscriptive character is also discernible in the temporal qualities of colonial law discussed by Pietro Costa.

From a methodological point of view, we find no predominance of conceptual dyads counterposing measurable profiles of the time factor, such as: continuity-change; tradition-modernity; crisis-stability; transition-revolution. Instead, it seems that the conceptual dyads, assuming an analytical value, are capable of exerting stress on the bonds between heterogeneous (but often simultaneous) characteristics of the time factor, such as for instance continuity-modernity or tradition-change; at the same time, conceptual combinations that highlight time States, such as transition-construction or transitioncirculation, take on importance in their own right.

Whereas the first approach, in which time has a descriptive role, orients the analysis toward a diachronic profile, the second, attentive to the adscriptive function of time, reveals the synchronic profiles of juridical experience.

Working with objects containing different historical phases (the circulation of legal knowledge through books, legal grafts of European origin in America, institutions founded on the past to legitimise new constitutional configurations such as uti possidetis, etc.) an analytical value is attributed to the transtemporality of law in the interpretation of the historical period under study.

At the same time, a further element of temporality - seldom considered in historiography - gains visibility: the coexistence of diverse temporal conditions in the historical-legal experience, namely the "acceleration of history» (i.e., the redefinition of the relationships between the past, present and future), on which Pietro Costa writes in describing the effect of colonisation in relation to temporality in the colonised territories.

The above example invokes, however, the integrated perspective of the factors space and time. Let us return, therefore, to the reflection on spatiotemporal interactions with some conclusive considerations.

\section{$4 \quad$ Spatiotemporality as a Challenge to Legal History}

Space and time are not only closely interrelated categories, but also practically indissoluble, which has led us to speak directly of spatiotemporality. This spatiotemporality, as the moment and place at which the two elements intersect, can be recognised by certain essential aspects. From the interaction 
between space and time arise conceptual categories that not only provide the basis for communities, collectives and societies, but also for modelling key components in forming the legal subjects who will examine the space and time in which they are inserted.

Such is the case of the status so masterfully expressed by Javier Barrientos, constructed from the notions of temporality and spatiality. The spatiotemporal conjunction is verified through the definition of societies (Costa), which are not only formed within spaces and times deriving from their own narratives, but, as in the case of nations, are inscribed within imaginary spaces and are the consequence of imaginary chronologies that are embodied in political representations (Costa).

In addition, entities such as States, whose existence stems from a marked redefinition of their place in time and of their time in space, need to invent other spaces and other times against which to object. Colonial experience comes into play in this dynamics, as the paradigm of a perfect conjunction of spatiotemporality, given that space becomes temporalized whereas space is spatialised: spatiality is materialised through temporality, insofar as the colonies' place lies in the past (Costa). Thus, the chronology imposed by the State as a body is so imperative that it creates a new, equally state-based chronology for other completely unrelated realities, which no longer respond to the challenges of their own space and time but form part of external spatiotemporal dimensions: those of Western States.

Historians will not cease to feel the inspiration and the challenge of the union of space and time. One of these challenges will be to uncover the terms of this conjunction through diverse historical experiences that, in the event, can be drawn toward present realities and projected into the future. This is the case of recovering complex spatiotemporal constellations that provide a plural environment for a reality that cannot aspire to be understood in monistic terms, such as the imagination of the ius commune which allowed attention to be paid to local specificity whereas the constellation of special features stood within a global space giving them unity as a whole within the universal (Agüero). In the same manner, certain patterns of thought are recoverable, such as the Schmittian «great spaces», which can be moulded into a global future of movement and constant self-definition (Cappellini).

But it is not merely a question of recovering, reformulating and re-using different appreciations of legal experiences in order to unravel and formulate 
the historical dimension of today's juridicities; there is also the possibility of envisioning new spatiotemporal categories, such as projected spaces (Costa).

All in all, striving to find the value of the spatiotemporal coordinates for legal history is equivalent to reconsidering both the thematic and methodological boundaries of the discipline. Legal history will have the capacity to produce useful knowledge insofar as it remains open to critical discussion of its own methodological rules, to reconsider its place within the scope of knowledge and to redefine its role in a global, perpetually changing world.

The new course set by this discipline, demanded by the social sciences, needed by future legal operators and which legal historians are destined to provide, will depend on our capacity to question ourselves, on the quality of our answers and on the courage with which we address the ensuing implications.

\section{Bibliography}

Armitage, David, Jo Guldi (2015), Le retour de la longue durée: une perspective anglo-américaine, in: Annales. Histoire, Sciences Sociales (Dossier: "La longue durée en débat") 70, 2, 289-318 (available at: http://dx.doi.org/10.1353/ ahs.2015.0033)

Cappellini, Paolo (2010), Storie di concetti giuridici, Torino: Giappichelli

Clavero, Bartolomé (2012), Gracia y derecho entre localización, recepción y globalización (lectura coral de Las Vísperas Constitucionales de António Hespanha), in: Quaderni fiorentini per la storia del pensiero giuridico moderno 41, 675-773 (available at: http://www.centropgm.unifi.it/cache/quaderni/41/ 0677.pdf)

Costa, Pietro (2004/2005), Il fardello della civilizzazione. Metamorfosi della sovranità nella giuscolonialistica italiana, in: Quaderni Fiorentini per la storia del pensiero giuridico moderno, 33/34, 169-257 (available at: http://www.centro pgm.unifi.it/cache/quaderni/33/0170.pdf)

Duve, Thomas (2012), Von der Europäischen Rechtsgeschichte zu einer Rechtsgeschichte Europas in globalhistorischer Perspektive, in: Rechtsgeschichte 20, 18-71 (available at: http://dx.doi.org/10.12946/gplh1)

Duve, Thomas (Ed.) (2014a), Entanglements in Legal History: Conceptual Approaches, Frankfurt am Main, MPIeR (available at: http://dx.doi.org/ 10.12946/gplh1)

Duve, Thомаs (2014b), European legal History - Concepts, Methods, Challenges, in: Duve (2014a)

Elias, Norbert (1989), Sobre el tiempo, México D. F.: Fondo de Cultura Económica 
Fonseca, Ricardo (2013), As Formas do Direito. Orden, Razão e Decisão, Curitiba: Juruá

Foucault, Michel (1986), Of other spaces, in: Diacritics, 22-27

Garriga Acosta, Carlos, Marta Lorente Sariñena (2007), La Constitución jurisdiccional, Madrid: Centro de Estudios Políticos y Constitucionales

Guldi, Jo, David Armitage (2014), The History Manifesto, Cambridge: Cambridge University Press (available at: http://historymanifesto.cambridge.org)

Hartog, François (2015), Régimes d'historicité: présentisme et expériences du temps, Paris: Seuil (available at: http://dx.doi.org/10.7312/columbia/ 9780231163767.001.0001)

Hespanha, Antonio Manuel (2013), Uncommon laws. Laws in the Extreme Peripheries of an Early Modern Empire, in: Zeitschrift der Savigny-Stiftung für Rechtsgeschichte, Germanistische Abteilung, 130, 180-194

Lorente Sariñena, Marta (2010), La Nación y las Españas. Representación y territorio en el constitucionalismo gaditano, Madrid: Ediciones UAM

Lorente Sariñena, Marta, José Maria Portillo (2012) (Dirs.), El momento gaditano. La Constitución en el orbe hispánico. (1808-1826), Madrid: Congreso de los Diputados

Marramao, Giacomo (2008), La passione del presente: breve lessico della modernitàmondo, Torino: Bollati Boringhieri

Meccarelli, Massimo (2015), The Assumed Space: Pre-reflective Spatiality and Doctrinal Configurations in Juridical Experience, in: Rechtsgeschichte 23, 241-252 (available at: http://dx.doi.org/10.12946/rg23/241-252)

Meccarelli, Massimo, Paolo Palchetti (2015a) (Eds.), Derecho en Movimiento. Personas, derechos y derecho en la dinámica global, Madrid, Universidad Carlos III de Madrid (available at: http://e-archivo.uc3m.es/bitstream/handle/ 10016/20251/derecho_HD33_2015.pdf?sequence=3)

Meccarelli, Massimo, Paolo Palchetti (2015b), Derecho en movimiento: una cuestión teórica nada convencional, in: Meccarelli / Palchetti (2015a), 9-19

ModÉER, KJELL Å. (2014), The deep structures of European normativity in a global context, in: Rechtsgeschichte 22, 275-281 (available at: http://dx.doi.org/ $10.12946 /$ rg22/275-281)

Paixão, Cristiano (2013), Tempo presente e Regimes de Historicidade. Perspectiva de Investigação para a História do direito, in: Fonseca (2013), 77-87

Raffestin, Claude (2013), Por una geografía del poder, México: El Colegio de Michoacán

RENN, Jürgen (2014), The globalization of knowledge in history and its normative challenges, in: Rechtsgeschichte 22, 52-60 (available at: http://dx.doi.org/ 10.12946/rg22/052-060)

Solla Sastre, María Julia (2015a), El gobierno de la especialidad. Personas y cuerpos en movimiento entre España y Ultramar (1850-1885), in: MecCarelli/ Palchetti (2015a), 21-71 
Solla Sastre, María Julia (2015b), Ultramar excepcional. La construcción de un espacio jurídico para España y sus colonias (1837-1898), in: Rechtsgeschichte 23, 222-238 (available at: http://dx.doi.org/10.12946/rg23/222-238)

Sordi, Bernardo (Ed.) (2013). Storia e diritto. Esperienze a confronto, Milano: Giuffrè

Turner, Frederick Jackson (2008), The significance of the frontier in American history, London: Penguin books

Vitucci, Chiara (2015), Some considerations on the two-way circulation of legal concepts and experiences between colonies and motherland, in: MecCARELLI / Palchetti (2015a), 97-105

Zimmermann, Eduardo (2014), Translations of the "American Model" in Nineteenth Century Argentina: Constitutional Culture as a Global Legal Entanglement, in: Duve (2014a), 385-425

$24 \quad$ Massimo Meccarelli, María Julia Solla Sastre 


\section{Experiences}





\section{A 'Spatial Turn' for Legal History? A Tentative Assessment}

\section{Introductory Remarks}

Our issue is to discuss the impact that the thematization of the space-temporal dimension has had (or could have), in general, on historical research and particularly on legal historiography.

Such a request risks seeming like an appeal to reinvent the wheel: it is selfevident that historians deal with time and space, inasmuch as they study phenomena which take place in temporally and spatially delimited contexts. We can admit that time and space are among the most complex and debated notions of modern philosophy, epistemology and physics, but we cannot take for granted that the philosophical definitions of time and space are a necessary prerequisite of the historian's daily job. We could assert that the historian, like the man in the street in his daily life, assumes the space-andtime categories in an immediate and unintentional way, without needing to provide solid definitions for them.

I think that the historian can do his job excellently without being compelled to explain the theoretical background of the tools of his trade, as a good craftsman handles his plane or his axe without thinking of their molecular structure. Nonetheless, a sharper awareness of our research strategies can contribute to their improvement and renewal and, in the worst case, it can prevent us from declaring, like Monsieur Jordain, that «for more than forty years we [spoke] in prose and [did not know]».

\section{The 'Spatial Turn' between Historiography and Geography}

The historian always deals with time and space. He/she, however, tends to assume time, rather than space, as the distinguishing feature of his/her disciplinary identity. It is time as the unit of measurement of changes that historians have taken into consideration in their empirical researches, as in 
their methodological enquiries. In comparison with the continuing attention to time, the analysis of space seems to be somewhat neglected by historians, and the question about the relationship between space and time appears even more disregarded. Two different, but contiguous disciplines, historiography and geography, seem to have implicitly adopted a convention about their specific fields: while time is the main concern of historians, space will be the preferred domain of geographers.

Of course, things are not exactly in these terms. In any case, a clue that such a simplification is not utterly misleading is that the intercourse between the two disciplines is usually not taken for granted, but marked as an interesting and innovative trial. From this standpoint, let us consider 'historical geography', which bears witness to the relevance assigned to diachronic analysis in geographic research and, respectively, to the role Lucien Febvre and Fernand Braudel conferred on geography within their proposal of a thorough renewal of historical methodology and of the very idea of history. And it is no coincidence that Febvre and Braudel pay unprecedented attention to the spatial dimension and, at the same time, are able to outline an original vision of the multiple 'temporalities' involved in the historiographical research.

Interdisciplinary intercourses between historiography and geography have also not been absent. Nevertheless, both disciplines lack in an adequate thematization of the spatial and, respectively, temporal dimension, and mainly of their interrelation. Indeed, not only historiography has been «space-blind»: ${ }^{\mathbf{1}}$ sociology itself has often undervalued the seminal role of the spatial-temporal coordinates (as Anthony Giddens remarks), even though some of its founding fathers have afforded a deep analysis of them. ${ }^{2}$

Geography perhaps, more than historiography, has showed a remarkable methodological anxiety and has sketched some interesting proposals, which flourished during the 1970s and 80s. Relevant suggestions came from the interpretation of Marx's thought put forward by the French sociologist Henri Lefebvre. His 1974 book, La production de l'espace, became a necessary step for a critical reassessment of geographical discipline. ${ }^{3}$ Not unlike Lefeb-

1 Castree (2009) 32.

2 Giddens (1987) 142: According to Giddens, the distinction between space and time «has helped among other things to reinforce disciplinary divisions».

3 Lefebvre (1976). 
vre, the English geographer David Harvey ${ }^{4}$ found in Marx's works the stimulus to discard his original positivistic approach and challenge a vision of space excessively influenced by Newton's physics.

In the modern (Newtonian and Cartesian) perspective, space was conceived as an objective phenomenon, existing in itself, independently from its contents. «In this sense, space was seen as a container that had effects on the objects existing within it, but was not itself affected by them». ${ }^{5}$ Indeed, Kant's Copernican revolution had reduced time and space to pure 'a priori' forms, to transcendental preconditions of human experience, but in any case geography's main frame of reference was still the Newtonian view of space (space as an hollow and inertial surface). ${ }^{\mathbf{6}}$

It is the implicit Newtonian bent of geography that David Harvey questions, stressing the opportunity of resorting to other, alternative views of space: Einstein's theory of relativity and, earlier, the relational theory, which David Harvey traces back to Leibniz. In an exchange of letters with the Newtonian Samuel Clarke, Leibniz had challenged Newton's idea of an absolute space, holding «Space to be something merely relative, as Time is», «an Order of Coexistences, as Time is an Order of Successions». ${ }^{7}$ Harvey's conclusion is that space is not an inertial surface on which social phenomena take place, but must be defined in strict connection with them: "processes do not occur in space but define their own spatial frame. The concept of space is embedded in or internal to process». ${ }^{\mathbf{8}}$

Space and social processes are linked by a mutual implication. According to Lefebvre, this assertion can be easily drawn from Marx's works devoted to the critique of political economy. Apparently, Marx had not deviated from the historicist tradition which gave preference to the temporal rather than to the spatial dimension of social phenomena. In fact, he had instead shown

4 Harvey (1973).

5 Cosgrove (2004) 58.

6 Di MÉo/BulÉon (2005) 22 ff.

7 Leibniz (1717): «As for my Own Opinion, I have said more than once, that I hold Space to be something merely relative, as Time is; that I hold it to be an Order of Coexistences, as Time is an Order of Successions. For Space denotes, in Terms of Possibility, an Order of Things which exist at the same time, considered as existing together; without enquiring into their Manner of Existing. And when many Things are seen together, one perceives That Order of Things among themselves».

8 Harvey (2006) 123. 
remarkable insight into the role of space in his analysis of the genesis and functioning of capitalistic society.

This is the lesson the new geography tries to learn from the historical analysis of capitalism: the opportunity of moving away from an idea of space as a hollow surface, as a simple container, unconnected with the social interaction that takes place 'in' it or 'over' it. The methodological cue that a renewed geography can draw from Marxian texts is the attempt to see space as an inner dimension of social phenomena. In turn, this implies the necessity to rethink Marx, avoiding leaving space to the mercy of the diachronic dimension. ${ }^{\text {? }}$

A critical rereading of Marx's works has also incited 'post-modern' geography to rethink space and its relationship with social dynamics and, at the same time, has prompted the other social sciences to make space a necessary step of their theoretical and empirical research.

Admittedly, both historiography (I have already mentioned Febvre and Braudel) and sociology included important insights into the spatial dimension (and its relationship with time). We can even maintain that sociology, between the nineteenth and twentieth century, truly broke with the Cartesian and Newtonian view of space, no less than with Kant's transcendental theory of space-time, which shared, despite all their contrasts, the same indifference to the impact of social dynamics and historical changes.

It is precisely this universalistic and meta-historic stance to which Durkheim opposes a socially influenced and historically differentiated sense of time and space. As a conclusion of a long ethno-sociological work, co-written with Marcel Mauss in 1903, he maintains that «des idées aussi abstraites que celles de temps et d'espace sont, à chaque moment de leur histoire, en rapport étroit avec l'organisation sociale correspondante» ${ }^{\mathbf{1 0}}$ and upholds the same thesis in Les formes élémentaires de la vie religieuse, published in 1912. ${ }^{\mathbf{1 1}}$ According to Durkheim, space and time are not abstract categories, but socially forged institutions, capable in turn of impinging upon individuals' actions. Space is not «ce milieu vague et indéterminé qu'avait imaginé Kant: purement et absolument homogène ...»; it is instead, like time, moulded

9 Soja (1989) $57 \mathrm{ff}$.

10 Durkheim / Mauss (1903) 72.

11 Durkheim (1912). 
differently according to the society of which it is a function. Space is not a homogeneous, constant and universal category, but assumes the contents determined by the culture and forms of life of a specific society. ${ }^{12}$ "Il existe des sociétés en Australie et dans l'Amérique du Nord où l'espace est conçu sous la forme d'un cercle immense, parce que le camp a lui-même une forme circulaire [...]. Ainsi, l'organisation sociale a été le modèle de l'organisation spatiale qui est comme un décalque de la première». ${ }^{\mathbf{1 3}}$ Indeed, every culture resorts to the same categories (time, space, causality and so on), but assigns specific and idiomatic contents to them. ${ }^{\mathbf{1 4}}$ Space and time must also be considered in the plural, as relative and multiple dimensions and not as universal and uniform categories.

Assuming space and time as social institutions is a methodological stance whose importance could hardly be overstated, considering its impact (direct and indirect) on twentieth-century sociology and historiography. Durkheim and the 'Durkheimians' (from Maurice Halbwachs to Georges Gurvitch) are correctly mentioned as the forerunners of a veritable «sociology of social times». ${ }^{15}$ Even looking outside the circle of the orthodox 'Durkheimians', such an important work as Norbert Elias', Essay on time ${ }^{\mathbf{1 6}}$ is hardly conceivable without referring to the Durkheimian 'turn'. According to Elias too, the vision of time changes depending on social contexts; it influences the civilization process and acts as a powerful instrument of social 'Disziplinierung', insofar as it is a social institution endowed with a relevant normative capability. $^{17}$

To tell the truth, Durkheim is not the only social scientist engaged in outlining a sociology of time and space. In the same years, Georg Simmel he too, a leading figure in nineteenth-twentieth-century culture - confronted the same issue in some essays, ${ }^{\mathbf{1 8}}$ which became his Soziologie, published in 1908. The importance of Simmel's reflection on space has long been undervalued and only the (relatively) recent reconsideration of space as a key

12 Durkheim (1912) 22.

13 Durkheim (1912) 23.

14 Schmaus (2004) 120.

15 Sue (1994). Cf. Lallement (2008).

16 Elias (1992).

17 Taвboni (2001).

18 Among them Simmel (1903) 27-71. 
concept in social sciences has stimulated a reassessment of his thought on relevant topics: ${ }^{19}$ from the critique of the 'traditional' idea of space to the social function of borders, from the connection between space and social groups to the importance of the migrations of peoples.

Important enquiries on time and space have been undoubtedly carried through by social and historical sciences between the nineteenth and twentieth centuries. It is also true, however, that over the long run, space and time have been taken for granted, more than investigated in order to determine their role and function in a specific research field. It is also not an exaggeration to say - as the custom is - that starting from the 1980s, a spatial turn has taken place: i.e. a renewed attention numerous disciplines (from sociology to geography and historiography) devote to the spatial dimension.

The leading figures of the 'spatial turn' come from several disciplinary traditions but share the conviction that the Newtonian view of a homogeneous space must be substituted by the monitoring of a multiplicity of different places. A place is not an indifferent point of the space, equal to every other point, but has idiomatic and irreplaceable features inasmuch as it is, at the same time, a product and a leverage of a social process. ${ }^{20}$

Michel de Certeau speaks of space as a "practiced place». ${ }^{21}$ Adopting a different terminology, but pursuing the same approach, many geographers refer to the 'territory' as a social and spatial phenomenon and explain 'territoriality' as the relationship between one or many social groups and the localities where they are settled. ${ }^{22}$ In an ethological perspective, the social dimension of territoriality is brought back to the animal instinct to defend their living space. ${ }^{23}$ Whatever the opinion about the ethological roots of 'territoriality', we are undoubtedly far from the Newtonian tradition, to which the 'new geographers' attribute two shortcomings: starting from a Newtonian view of space, on one side, social processes are described independently from their 'localization' and, on the other side, territory is conceived as a simple 'natural' entity, understandable even if we leave aside its

19 Glauser (2006); Ethington (1997); Marramao (2005); Marramao (2013).

20 Withers (2009) 641.

21 De Certeau (1984) 117.

22 Di MÉo/BulÉon (2005) 77 ff.

23 Ardrey (1966). 
involvement in social processes. ${ }^{24}$ "While simplistic, it is not misleading to say that, in the current discourse, 'place' is good and 'space' is bad». ${ }^{25}$

The 'spatial turn' also induces several disciplines to rethink some vital aspects of their frame of reference, such as the notion of space and the relationship between the temporal and the spatial dimension. Though both historiography and geography can share this trend, its effects are different according to their respective traditions. As for historiography, usually concentrated on diachrony, the 'discovery' of space implies the attempt to understand «what happens when historical processes are conceived and described as spatial and local processes». In short, the question is: «what happens if we 'think jointly' history and its places?». ${ }^{26}$ As for geography, respectively, it is the temporal dimension which is acquiring an increasing relevance in the analysis of socio-spatial phenomena. ${ }^{27}$

Different disciplines also try to avoid the misleading shortcut consisting of the contrast between time and space, as though time were involved with movement and changes, and space with an immobile and frozen reality. Time and space are intertwined: the diachronic development of social phenomena is possible insofar as they 'have place', are 'located' somewhere and, vice versa, places are moulded by social processes understandable only in the frame of time. A clue that we must take the space-and-time connection seriously is that language usually resorts to spatial metaphors in order to represent time (time as a line; future as something 'ahead of us' and past as something 'behind us'). A 'spacing history', a historiography capable of focusing on the spatial dimension of change, is only the other side of an analysis of space as a 'timed space', a galaxy of places marked by the long sedimentation of history.

\section{A 'spacing history': specimens and hypotheses}

What are the improvements a 'spacing history' affords to the frame and the instruments of the historical research? How can a better awareness of spatial and temporal coordinates sharpen the cognitive instruments of the histor-

24 Soja (1989) 76-77.

25 Ethington (2007) 481.

26 SCHLÖGEL (2003) 9-10.

27 Pacelli (2007) 15. We can refer, on one side, to the historical geography and, on another side, to the time-geography cultivated by Torsten Hägerstrand and the Lund School. 
ian? Of course, I cannot offer a complete survey of the issues the 'spatial turn' involves. I can simply provide some cursory references to the changing attitude of historiography towards the spatial dimension of its research field.

It may be convenient to resort to a probably fragile, but not unfounded distinction: the distinction between what I should like to call the 'lived' space and the 'imagined' space. Before explaining the meaning of this distinction, I must refer to a logically previous distinction: the distinction between the metalanguage and the object-language.

The historian's metalanguage is the set of the conceptual instruments he / she employs in order to understand and report some aspects of the past. It is in the moment in which the historian arranges his/her metalanguage that he/she can choose to adopt one or another definition of space. The Newtonian, Leibnizian, Einsteinian or post-modern vision of space provides the linguistic-conceptual instruments the historian uses to understand the past. The definitions of time and space, whatever they be, belong to the tool-kit of the historian: they are something by means of which, and not something about which, he / she speaks.

What the historian speaks about, the object of his/ her research, is one or another society developing in the horizon of space-and-time. It is to the object of the historian's research that we must refer the abovementioned distinction between a 'lived' space and an 'imagined' space.

On one side, a society exists insofar as it is 'localized', capable of conjointly transforming itself and its space. Space is also an intrinsic component of social dynamics: it is a 'lived' space. On the other side, the cultural representation that the same society offers of itself most probably includes its peculiar vision of space and time. In this sense, space is not only 'lived', but also 'represented', 'imagined'. We must however underline the provisory and expository scope of this distinction, inasmuch as 'lived' and 'imagined' spaces are in fact so intertwined that they get mixed up.

\subsection{The 'lived' space}

If we glance at political, social and legal phenomena using the temporal-andspatial coordinates as a lens, as an optical device, in order to focus on the investigated reality, we notice that it can be directed towards the extremely small or the extremely large: in other words, it can be indifferently used as a microscope or as a telescope. 
When Febvre and Braudel underwrote the cooperation between historiography and geography, relying on the interconnection between space and time, and achieved the distinction among different historical times, diverse rhythms of social and cultural change, they put the spatial-temporal lens at the service of that histoire totale, which was their ultimate goal (a decisive, though probably unattainable goal). ${ }^{28}$ In any case, the aim of an integral, as far as possible 'total', knowledge of the past can be pursued by using not the telescope, but the microscope and focusing on the 'micro' level of historical processes, as shown by a recent, and methodologically shrewd, social history. Indeed, the question of what 'local' means in the «local history» remains open. ${ }^{29}$ Doreen Massey (a key spokesperson of the geographical 'nouvelle vague') notes that places are "constructed out of articulations of social relations $[\ldots]$ which are not only internal to that locale but which link them to elsewhere. Their 'local uniqueness' is always already a product of wider contacts; the local is always already a product in part of 'global' forces, where global in this context refers not necessarily to the planetary scale, but to the geographical beyond, the world beyond the place itself». ${ }^{30}$ In any case, what a 'spacing history' demands is that its followers, whether they use the microscope or the telescope, focus their efforts on the "production of locality» (as Appadurai puts it). ${ }^{31}$

\section{a) Space in the Middle Ages}

Medieval society is an extraordinary stage in order to attend to a peculiar process of 'production of locality'. A great portrayal of it is provided by the fascinating and enlightening work by Paul Zumthor ${ }^{32}$ (curiously neglected by legal historiography, if I am not mistaken). I refer the reader to it overall, but I cannot escape from quoting some passages.

«Man in those times - as the Swiss philologist writes - does not believe, as we do, in the existence of a 'material' reality, not human and separated from

28 An interesting assessment is offered by Сомва (1981). Recent and important contributions are provided by Blanco (2008).

29 Driver/Samuel (1995) 5.

30 Massey (1995) 183.

31 Appadurai (1996) 182: «Locality as a phenomenological property of social life, a structure of feeling that is produced by particular forms of intentional activity and that yields particular sorts of material effects».

ZuMTHOR (1993). 
us [...]. Everybody cultivated, in his relationship with the land, a warm complicity, which we have lost and is for us now almost inconceivable. For a medieval farmer, as for a medieval citizen, lord or clergyman, space had nothing to do with our notion of it: a three-dimensional and uniform entity, divisible in equal parts and endowed with features independent from its material content. Medieval space is neither abstract nor homogeneous [...]; it is less perceived than lived». ${ }^{33}$

The most different social and political relationships «are spatialised, and every space tends to become the signifier of a social meaning». ${ }^{34}$ Space coincides with a galaxy of places; and a place is «laden with a positive, firm and rich sense: $[\ldots]$ it is the portion of land on which human beings live [...]. A place cannot be divided in parts, because it unifies all its constitutive elements and relationships». ${ }^{35}$ «Personal identity cannot be separated from the appropriation of a place and the adaptation to the immediate environment». ${ }^{36}$ It is the radical 'localization' of every individual and collective experience which "creates the 'territory', the civilized space of everyone, who has occupied it by his work and has imposed his law to it». ${ }^{37}$ It is territory which «contains the history of the human beings who have created it and live by the means of it [...]: it embeds the symbolic intention of a struggle against death». ${ }^{\mathbf{3 8}}$

I rely on the reader's tolerance for these long quotations. I hope they will contribute to show the relevance of space for medieval culture and the urgency of a 'micro-historical' approach capable of affording specimens and tests of such a peculiar vision of space. It is in this perspective that Angelo Torre suggests studying places «as continually rebuilt social and cultural structures»: ${ }^{39}$ places of a discontinuous and not homogeneous space (a «chifonné» - a crumpled - space, to borrow the term from the cosmologist Luminet); places as microcosms in which the social macrocosm is reflected and mirrored.

33 Zumthor (1993) 33.

34 Zumthor (1993) 40.

35 Zumthor (1993) 49.

36 Zumthor (1993) 50-51.

37 ZuMThor (1993) 75.

38 ZumTHOR (1993) 76-77.

39 Torre (2011). Cf. Costa (2012). 
b) Iurisdictio and territorium

It is in this peculiar (and, for us, exotic) galaxy of places that we must situate legal institutions and doctrines (to which our usual conceptual distinctions first of all, the dichotomy between public and private law - cannot be mechanically referred).

Medieval 'lived' space is the social and cultural environment in which the theory and the practice of dominium, such as outlined by Paolo Grossi, take place. According to Grossi's penetrating insight, the medieval sense of proximity and mutual relationship between human beings and material things «in the frame of a superior order which encompasses human beings and things in an unitary vital organization» ${ }^{\mathbf{4 0}}$ can be explained resorting to the socio-anthropological concept of 'participation', outlined and applied by Lucien Lévy-Bruhl and Marcel Mauss.

The theory and practice of dominium are only an eloquent specimen of the intimate relationship between a legal institute and a thick texture of places which cannot be understood in the frame of a Newtonian or Cartesian view of space. From a more general standpoint, it is the whole government of society which must be rethought highlighting the marks impressed on it by the medieval sense of space. This is the approach adopted by António Manuel Hespanha, who in an essay published in $1982^{41}$ examines the relationship between political powers and territory during the 'Ancien Régime'. Its basic idea is to go beyond a naturalistic, objectivistic and universalistic idea of space and stress its culturally influenced and relativistic content. In pre-modern societies, space is a multiple, fragmented and uneven entity, which influences and moulds political theories and practices.

Hespanha's stance was patently ground-breaking: it is difficult to find, in the legal-historical literature of that period, a comparable attempt at focusing on the relevance of the spatial dimension and consequently building a bridge between geography and the history of political institutions.

According to Hespanha, the pre-modern political space was a «miniaturized» space: a fragmented and uneven space, where face-to-face relationships and oral traditions were prevalent; a space which coincided with the life of the group and moulded all its political and legal forms. ${ }^{42}$ Luca Mannori, in a

40 Grossi (1968) 93.

41 Hespanha (1982).

42 Hespanha (1982) 478-482. 
recent essay, refers to a «legal saturation of space», which «immediately reacts on the vision of power and intensively moulds its morphology». ${ }^{43}$ Spatial and political-legal dimensions are complementary and determine the representation of the iurisdictio (i.e. the vision of power and government in medieval culture).

A widely quoted formula in medieval jurisprudence is the following: «iurisdictio cohaeret territorio». Jesús Vallejo (in his book devoted to the normative power in medieval legal doctrine) had also conveniently focused on the link between iurisdictio and dominium and iurisdictio and territorium. ${ }^{\mathbf{4 4}}$ The basis of such a connection relies precisely on the medieval vision of space that Hespanha had assumed as the keystone of his historical analysis. How deeply spatial coordinates affect our understanding of the connection between iurisdictio and territorium has been recently confirmed by an original and important research by Paolo Marchetti, whose attention to geographers is still an unusual strategy within legal historiography. ${ }^{45}$

Even on the strength of only these few references, we can realize that the spatial dimension has not been ignored by legal historiography. On the contrary, we must bear in mind that medieval studies have been working for a long time on the relationship between political institutions and territory. Suffice it to refer to Pietro Vaccari, Emilio Sereni, Cinzio Violante and Giovanni De Vergottini, to mention only some Italian scholars. ${ }^{46}$ The 'spatial turn' is not at all the unpredicted discovery of an unknown land. It is however true that in the past, numerous and valuable historiographical outcomes ran the risk of missing their target because unsupported by an adequate general vision and methodological awareness. On the contrary, a sharper understanding of spatial and temporal coordinates, promoted by the cooperation of different disciplinary strategies, can be the right frame of reference for a proper placement of historical data.

43 MANnORI (2008) 44-45.

44 Vallejo (1992) 128-152.

45 Marchetti (2001).

46 Cf. by way of example Vaccari (1920); Sereni (1961); De Vergottini (1977); Spicciani / Violante (1997-1998). It would be interesting, but demanding, to make an assessment of the references (implicit or explicit) to spatial issues in medieval and modern historiography. Cf. Salvemini (2006). 


\section{c) The Newtonian Space and the Modern State}

The medieval past and its political and legal theories and practices must be rethought, moving from an idea of space finally exempted from the charges of the Newtonian tradition. The historical analysis of a major phenomenon such as the medieval city can benefit from a clear understanding of its peculiar spatial dimension. The medieval and proto-modern city is an emblematical 'production of locality': it withdraws within its circle of walls and, at the same time, aims for a further extension of its power and influence; it lives inside the polymorphic and miniaturized space of medieval society, but contributes to the making of a different political landscape. ${ }^{\mathbf{4 7}}$ The city creates its own space and assumes this as a symbol of its identity. In this regard, the distinction (which I have proposed faut de mieux) between the 'lived' and the 'imagined' space appears more than ever fragile: the medieval city is conjointly urbs and civitas, a city of stones and a city of men (as suggested by Isidore's famous definition), a centre of power and a cohesive community. According to medieval jurists, from Cynus to Bartolus, the city as universitas is the holder of the iurisdictio. It is the city (as Luca Mannori suggests in the wake of Hintze), the city as populus or communitas, which can be assumed as the core of a socio-political model, the 'corporatist' model, according to which the organization of the territory proceeds «from what is 'small', and not from what is 'big', from the 'part' and not from the 'whole'». ${ }^{48}$

If we focus on the spatial dimension, we are able to rethink medieval phenomena in an original perspective and gain new insights into the genesis of modern sovereignty. In this regard, the hypothesis of a correspondence, or even of a mutual implication, between the ('Newtonian') vision of space and the development of new political institutions could be proposed. Indeed, only a great deal of accurate and targeted researches could afford the indispensable evidences. If anything, it is easier to find some intuitive confirmation of the hypothesis if we glance at the arrival point of the modernization process (more than at its intermediate passages).

It is a matter of fact that, first, a new sovereign power arises (at different times, depending on the several geographical areas, but with shared lines of 
development everywhere): it is a power which aims to have efficient armies at its disposal, requires an increasing amount of money for this purpose and tries to obtain an extensive control over society. Secondly, a new economic pattern takes shape: industrial capitalism. Industrialism transforms (as underlined by Lefebvre's reinterpretation of Marx) the living experience of temporality inasmuch as it assumes the working time as an arithmetically divisible measure on which the value of the performance depends. Thirdly, the new sovereign power - the modern State - aims to render its territory as uniform and homogeneous as possible, in the name of a 'rational' organization and control of it.

For the new State, all the elements of the territory (all the points of the surface) are perfectly equivalent. State and territory are closely connected, according to a legal doctrine that continuously underlines the following dogmas: every political organization must be defined as a State (as an actual or as an emerging State); and every portion of space must be considered as a homogeneous and divisible entity actually or potentially assigned to one State or another. ${ }^{49}$

Jurists, scientists (from Galilei to Newton) and geographers have afforded seminal contributions to a revolution which involves, at the same time, the vision of space, the form of political power and the organization of territory. The development of a 'scientific' cartography is an indispensable requisite for an efficacious control over territory: «a map is as a device which reduces the dimension of the world and perfectly matches the needs of a modern territorial State, which proves itself as the spatial model for the organization of politics and economy, even before the bourgeois revolutions». ${ }^{50}$ Not coincidentally, a central issue of present geographical research is the establishment of the State as the exclusive controlling unit in the territory. ${ }^{51}$

At the dawn of modernity, the 'lived' space also corresponds to a 'Newtonian' stance, as much as the ongoing political process takes the shape of

49 Mannori (2008) 58: «Quelle leggi dell'89, di cui il Sieyès citato all'inizio fu uno dei massimi ispiratori, producono per la prima volta un territorio davvero 'tutto uguale', dal quale è stata sradicata perfino ogni minima differenza tra città e campagna, e le cui articolazioni sono costituite ed operano in base ad uno statuto di fonte esclusivamente statuale».

50 Minca/Bialasiewicz (2004) 85; Harley (1988) 57-76. Cf. Ruschi (2012) 206-212.

51 A renown examples is Taylor (1994). Cf. Brenner (2003); Belina/ Michel (2011). 
the State. 'Newtonian' space and the State's organization mutually imply themselves as outcomes of the same historical process. Interestingly enough, this connection has been maintained for a long time. It was a 'received view' of the scientific community assuming both State and space as 'natural', ahistoric phenomena: politics were made coincident with State and space was conceived as a hollow and homogenous surface. These statements have been assumed for a long time by historians as the conceptual tools (the 'metalanguage') of their researches. On the contrary, the 'spatial turn' stresses the historical connection between the modern State and the 'Newtonian' space and, accordingly, offers the opportunity to rethink both politics and space without assuming their 'modern' representation as the unchangeable and binding conceptual background of historiography.

d) The problem of borders and 'diffusion geography'

The 'spatial turn' enables us to focus on two peculiar 'idola' of modernity - a State-centric vision of politics and a Newtonian view of space - and is therefore well equipped to enlighten a strictly connected issue in a new way: frontiers and borders.

At a first glance, we could take the gap between the medieval and modern ages for granted and assign to the latter a rigid setting of borders and a sharp contrast between the 'inside' and the 'outside' of a political community. Indeed, it is reasonable to expect that in the fragmented and uneven medieval space, the differences between what is near and what is distant and alien are less absolute and incontrovertible than in a 'Westphalian' scenario, marked by the sharp divisions among legally equal sovereign States. It is however true that a good crop of historical, geographical and anthropological researches invite us to question the received view of the impermeability of borders even at an advanced stage of modernity. ${ }^{52}$

An efficacious metaphor refers to borders as 'porous' walls: borders, even if conceived and organized in different ways in different contexts, actually acted not as impenetrable bulwarks, but as sponges, which rejected something, but absorbed something else and poured it inside. Borders can also be

52 Marchetti (2001) 40. Cf. Van Houtum (2005). An interesting, inter-disciplinary approach in PAstore (2007). 
considered as devices which separate contiguous spaces and, at the same time, as places where goods, human beings, languages, doctrines, norms and institutions pass through. Borders are, at the same time, a spatial division and an intersection of an intense social dynamics.

Geographers have focused on this phenomenon resorting to the concept of 'spatial diffusion': the movement of human beings, goods, ideas or even of viruses and diseases, which takes places in space and time. Some 'diffusion geographers' have tried to outline some abstract models of this 'double' movement. ${ }^{53}$

To be sure, the movement of viruses in space and time seems to be an utterly exotic issue for a legal historian. In any case, it is undeniable that the historian of political institutions and doctrines is deeply conversant with the movements and transfers of elements much closer to his/her domain: we can take our pick from an extensive list of legal (normative and doctrinal) texts continuously moving through time and space (the most famous specimen, ça va sans dire, is Corpus Iuris). The legal historian resorts in this case not to the concept of 'diffusion', but to a different, and undoubtedly seminal, hermeneutical category: the idea of reception. It would be therefore important for legal historiography to rethink the concept of reception in the light of recent literary theories (starting from the contributions of Robert Jauss). An essential aspect of legal culture and practice is the inexhaustible web of texts and interpretations, which is the core of a reception theory, while a methodological adventure in the realm of diffusion geography could seem to be adventurous and risky. Nevertheless, it is difficult to understand a text that moves from its original context and takes new roots in a different interpretative community without focusing on space and time. We could also expect interesting achievements from the synergy of methodologies which, despite their different background, share the same attention to the spatial and temporal dimension.

\section{e) The heterotopies}

Borders are, at the same time, places of separation and passage. At first glance, they seem to coincide with the divide between different political communities, but this claimed coincidence depends on a vision of politics

53 A critique of the link between eurocentrism and 'diffusionist' theories in BLaUt (1993). 
and space which identifies political power with the State and assumes space as a 'natural' and objective entity. In fact, borders (and the consequent existence of differentiated spaces and the connected dialectics of 'inside' and 'outside) affect not only the periphery of a society, but even its intrinsic arrangement.

The sovereign power and the nation-State do not exhaust the space of politics and society. Other powers and other spaces do exist. Not a geographer, but a philosopher - Michel Foucault - has drawn our attention to them. Foucault explicitly devoted only a few (but enlightening) essays to space, but was always mindful (in his 'major' works) of the spatial and temporal dimension, indeed so much that the 'new geography' has gleaned important suggestions from his books and from the lively dialogue with him. $^{54}$

In a short essay of 1984, Foucault speaks of «espaces autres», of 'other' space, of «heterotopies». ${ }^{55} \mathrm{He}$ is clearheadedly aware of the different visions of space and time and considers «a fatal interlacement between time and space» as a peculiar aspect of Western history. Galilei's and Newton's scientific revolution suggested the idea of «an infinite and infinitely open space», in contrast to the pre-modern space which was «the space of localization». ${ }^{\mathbf{5 6}}$ Instead, we are becoming aware that our experience of space is not uniform, but essentially heterogeneous.

In this perspective, Foucault invites us to consider space in the plural: i.e. to go beyond the nineteenth-century vision of sovereignty and its unitary political space, and to outline an uneven landscape of powers, a multiplicity of places, which must be conceived not as hubs of a de-spatialised social interaction, but as socially forged places. Utopias too are «espaces autres». They are however, unreal places, while we can find different but real spaces inside society, different places, «espaces autres», which are «a somewhat mythical and realistic contestation of the space where we live». ${ }^{57}$

Society is represented as a multiplicity of variously separated and connected places. Among them, the «espaces autres» are autonomous micro-

54 Let us refer to the dialogue with the geographers of «Hérodote». About the intercourse between Foucault and the geographers cf. Crampton/ELden (2007).

55 Foucault (1994).

56 Foucault (1994) 20-21.

57 Foucault (1994) 25. 
cosms, at the same time strictly linked with the social macrocosm. An extended research field opens up, starting from those 'different' places, whose geography Foucault has brilliantly outlined: the places of punishment, of detention, of industrial production, Bentham's panoptical imagery and the numerous variations on the theme it suggests.

The panoptical prison is however, only one of the existing heterotopies. The category of the "espaces autres» is a key that can open many doors, employable wherever the social production of a place comes into play. A long list of examples from the most disparate historical contexts could be mentioned. Let us think about spaces that separate and segregate social groups from the community to which they belong: the Jewish ghetto (throughout the course of its history until its tragic conclusion) is an emblematic, though not the only possible example. And even the medieval immunitas can be described (according to Barbara Rosenwein ${ }^{58}$ and Angelo Torre ${ }^{59}$ ) as a practice which results in the establishment of a space exempted from the intrusion of powers: a space 'autre', capable, at the same time, of confirming and contradicting the existing order.

f) The space of colonization

The scenario where powers and places are situated is also more complex than the nineteenth-century theory of State supposed, taking for granted that societies were homogeneous realities, neatly delimited by rigid boundary lines. A further complication arises if we consider what happens beyond the area of the State's sovereignty. For every State, the space controlled by a different sovereign State is an exterior space. The world however does not coincide, in the modern era, with a network of States. A second boundary emerges, which separates the ius publicum europaeum from the 'outer' world, Europe from its 'others', the West from the colonized peoples.

In the colonization process (which is not an event among others, but the very horizon of modern history), power and space, geographic knowledge and political and legal theories are strictly connected: the enlargement of space for Europe (the so-called geographical discoveries) keeps pace with the subjugation of the new world; geography develops in tune with the needs of

58 Rosenwein (1999). Cf. Latini (2002).

59 TORre (2011).

$44 \quad$ Pietro Costa 
colonization, and the theory and the practice of sovereignty are rethought in order to control incredibly large, differentiated and complicated areas.

A huge research field opens up, where the link between power and space can be assumed as the guideline to reassess the colonization process. Such a process is something like an epochal short-circuit among different political spaces and is, at the same time, a destructive and transformative agent of 'production of places'.

In this perspective, the concept of territoriality can be helpful. Geographers have devoted increasing attention to it, starting from the 1970s-80s. ${ }^{\mathbf{6 0}}$ Territoriality involves - as Robert Sack writes - the will to influence or control a geographic area and can be defined as «the attempt by an individual or group $(\mathrm{x})$ to influence, affect, or control objects, people, and relationships (y) by delimiting and asserting control over a geographic area». ${ }^{\mathbf{6 1}}$ Moreover, this notion is relevant, according to Sack, for the analysis of sovereignty. ${ }^{62}$ The approach of the Swiss geographer Claude Raffestin ${ }^{63}$ is different, more concerned with the 'relational' dimension of the principle of territoriality, but not less influential, mainly in the francophone and European-continental area. In our perspective, it is interesting to go beyond the differences between these two approaches and endorse their convergence: ${ }^{\mathbf{6 4}}$ the proposal to assume territory not as a natural, merely physical object, but as the material and symbolic outcome of a social interaction imbued with the dialectics of power and resistance.

Colonization can be considered in the light of the principle of territoriality. We are faced with a process which coincides with innumerable (collective and individual) acts of a symbolic and material appropriation of space. Territorialisation is a process which erases or deeply changes the pre-existent cultural and economic reality and replaces it with new powers, values and ways of life. It is a process which de-territorialises the space and, at the same time, re-territorialises it and is never predictable, linear and mechanical, but the result of continuous conflicts and 'negotiations'.

60 Turco (2010). Interesting considerations on medieval history in Somaini (2012).

61 SACK (1983) 56.

62 SACK (1983) 55.

63 Raffestin (1980); Raffestin (2007).

64 Murphy (2012). 
Colonial theories and practices could also be examined taking into consideration the interlacement between space, power and discourses referred to by the principle of territoriality. In the colonization process, however, not only space comes into play: space and time are strictly connected.

First of all, colonized societies experience time according to cultural standards, which are quite different from those adopted by modern Europe: the abstract, linear and divisible time familiar to European modernity is at variance with differently conceived 'temporalities'. In the de-territorialisation and re-territorialisation process, time can perform two different, but complementary tasks: on one side, it is a symbolic resource and an identity mark of the colonizers' culture and, on the other side, it serves the purpose of controlling and disciplining the life and work of subjugated peoples.

Secondly, Western culture resorts to a peculiar interlacement between space and time in order to represent the colonized world. According to the colonizers' culture, the colonized space is not a 'contemporary' reality, but belongs to a different temporality, to a distant and archaic age. «Different 'places' - as Doreen Massey writes - were interpreted as different stages in a single temporal development. All the stories of unilinear progress, modernization, development, the sequence of modes of production [...] perform this operation. Western Europe is 'advanced', other parts of the world 'some way behind', yet others are 'backward'. 'Africa' is not different from Western Europe, it is (just) behind». ${ }^{65}$ What is distant in space is thrown back in time, in a primitive stage of history, whose climax and accomplishment are supposed to coincide with Western modernity.

Western philosophy of history - the idea of history as progress, as a transit from savagery to civilization, from the archaic darkness to the light of modernity - is not only an idea of time, but implies and presupposes a precise and rigid differentiation and hierarchisation of space.

g) The shrinkage of space-time and the acceleration of history

Both spatial and temporal coordinates are employed by nineteenth-century philosophy of history in order to keep the metropolis at a safe distance from colonies and stress the radical, qualitative differences which separate the former from the latter. 
A different use of the space-time connection is however, available. The space-and-time dimension can be assumed as an indicator of the direction of development of Western history. In this perspective (adopted by prominent historians and sociologists in a relatively recent period), the space-time connection becomes one of the most relevant marks of the modernization process. The transition to modernity comes up beside a different experience of time and condenses in a key-word: 'acceleration'. The development from the ancient and medieval world to our present can be represented under the banner of an increasing speed.

The uneven and uncertain space of the Middle Ages cannot be detached from the slow rhythm and scansion of time. The turning point must be situated in the age Reinhart Koselleck defined as a Sattelzeit: a period (from the second half of eighteenth century to the first half of nineteenth century) intermediate between the pre-modern age and accomplished modernity, when a new vision of time arises and the past loses ground to the benefit of future. The ancient (Aristotelian and pre-modern) idea of a static, 'natural' and unchangeable time is replaced by the view of an unavoidable and compelling rush toward the future. ${ }^{\mathbf{6 6}}$

Modernity looks to the future and discovers speed: the rhythm of life accelerates, distances shorten and space and time contract. Modernity moves closer to post-modernity within the guidelines of an increasing acceleration. According to the sociologist Harmut Rosa, acceleration is already perceivable in the early development of the modern State; ${ }^{67}$ it takes hold during the second industrial revolution (when the means of transport and communication dramatically change) ${ }^{68}$ and becomes the dominant mark of twentieth century and the third millennium. Paul Virilio has introduced a neologism 'dromologie' - to label the scientific analysis of the increasing speed of processes and decisions in contemporary society. ${ }^{69}$ In the global village exalted by present 'globalization philosophies', space contracts and time is inclined to coincide with the instant. Distance in space and distance in time (and also past and future) become meaningless and all seems to become simultaneous and ubiquitous.

66 Koselleck (1979).

67 Rosa (2005) $311 \mathrm{ff}$.

68 KERN (1983).

69 Virilio (1977). Cf. LeCCARdi (2009). 
Whatever the reliability of such statements, the widespread awareness of a spatial-temporal 'revolution' provoked by globalization is likely to break in the secluded laboratory of the historian (and of the legal historian), suggesting the advisability to enrich his/her metalanguage with new questions, if not with new tools.

\subsection{The 'imagined' space}

Space and time are the socially and historically predetermined condition, which renders our experience possible. What I have termed the 'lived' space is the spatial component of social interaction: places are not the sections of a merely physical space, but are determined and forged by social practices. In this process of social appropriation of space, symbols and discourses have a major role and, on this ground, the proposed distinction between 'lived' and 'imagined' space has the purpose of mere orientation. What renders this distinction somewhat reasonable is that a society can hardly experience space and time without developing some (more or less sophisticated) discourses about them (in Western culture, time has become - from Augustine to Heidegger - one of the key-issues of philosophical investigation). This is evident and undisputable. It is perhaps less trivial to remark that a specific vision of space has possibly supported the development of a legal theory or of a political ideology and that, vice versa, a political and legal doctrine has melded with some vision of space.

a) Building identities: from the city to the nation

The 'lived' space is an endless production of places and a material and symbolic appropriation of territory. The embedment of a social group in a specific place usually increases its sense of cohesion, but not necessarily and does it immediately translate into the symbol of a political identity. Let us consider the meaning of 'natio' in medieval society. Natio refers to the geographical origin of a group and connects it with numerous identity traits (language, usages and so on), but it does not include a precise political meaning: for instance, the students in the University of Bologna were gathered with reference to the nationes (or subnationes) to which they belonged. ${ }^{70}$ 
The importance of a territorial embedment is undeniable, but its role in the development of a political identity is not immediate and obvious. ${ }^{71} \mathrm{~A}$ medieval example of a new political identity has to do with the changes which affect the cities, starting from the twelfth century. It is in the medieval city that 'lived' and 'imagined' spaces are so strictly interlaced that their distinction appears uncertain and blurred. The medieval city (a cohesive and hierarchical community) implants its image in the space, cluttering it with meaningful places, which are symbols of its power and of its attractive force.

Experience and symbols are strictly connected. The Laudes urbis, the writings that exalt the beauties of one city or another, praise the richness and greatness of the city and of its palaces, the firmness of the walls, the fertile and pleasant land which surrounds it (and often implicitly or explicitly recall the urban place par excellence, the archetypical city: Jerusalem). The power and greatness of the city are celebrated by discourses and, at the same time, are written on its stones, on the territory, walls and boundaries, and all these material and symbolic marks cooperate in creating an uncompromising collective identity.

Space is a symbol of identity and, at the same time, the evidence of the supremacy of the city. This latter aspect is considered by medieval authors, but is still more underlined by Machiavelli, who emphasizes the effects produced by the territorial expansion of the city on the preservation of its political order. Human nature and its libido dominandi prohibit from supposing that a political regime can go on indefinitely without increasing its power and enlarging its territory. The spatial parameter becomes an essential point of reference in order not only to understand the pattern of a political community, but also to value its chances to survive. According to Machiavelli, the stability of a respublica cannot be separated from its territorial expansion. The time of the city is measured by its spatial enlargement.

The territorial dimension of a political regime impacts on its structure (for instance, according to Montesquieu, only a little State can take the shape of a republic ${ }^{\mathbf{7 2}}$ ). Little political communities are however a relic of the past. The prevailing political organization in the eighteenth and nineteenth centuries is a big State which aims to control every single portion of its territory,

72 Gabba/Schiavone (1999). 
assuming space as a homogeneous and divisible entity. The 'lived' space of the State seems to perfectly match with the Newtonian or Cartesian view of space. It is exactly this vision of space that jurists presuppose when they assume territory as one of the three essential components of the State. In this case, there is no tension at all between the 'imagined' or 'represented' and the 'lived' space: the theoretical (Newtonian) view of space perfectly coincides with the government strategies of the State.

The development of the big States seems to overshadow the symbolic dimension of space: the link between the localization of the city, its territorial embedment, and the sense of a collective identity seems to belong to the remote era of medieval communes. A new (and increasingly employed) term however, reshuffles the cards: 'nation'. Nation was already a current expression in the age of absolute monarchies, but it runs into a dramatic semantic change during the nineteenth century. ${ }^{73}$

In the frame of the big nineteenth-century States, nation performs a task analogous to what the city accomplished in the Middle Ages: nation is symbol of unity, inclusion and belonging. Whenever it appears as coextensive with the State, it is able to pour the warm stream formed by shared identity and community into the cold process of government, in the rigid hierarchical relationship between State and citizen.

Nation continues the game started by the city in a different field. In the city, the connection between communitarian identity and space was immediately perceivable: the city was composed of its citizen and its stones, it was at the same time a physical and an ideal entity, which exhibited its political identity and its spatial roots with its simple being. Nation is different: unlike the city, it cannot point at the stones and the places in which it materialises. Nation (even more than the city) needs however, to gain its peculiar spatial dimension: it is by territory that it can become concrete and 'visible'. In nineteenth-century literature, the nation's tokens increase in number: language, history, ethos and a common destiny are repeatedly mentioned as essential components of a collective identity. A further and ultimate element must however, be launched: territory. It is the territory which identifies a political community and distinguishes it from another. Territory, as a component of nation, is not however an indifferent and fortuitous part of the 
world, but the space history and destiny have intimately connected with a community as an inseparable part of its identity. Nation is anchored to the soil: its territory is labelled as 'natural' in the nineteenth century. Territory renders the nation visible, connects it to the State and coincides with the area on which the State wields its power. ${ }^{74}$

The establishment of a national political identity presupposes a symbolic usage of space and at the same time implies a precise vision of time. The nation space is linked with a long-lasting time, which recalls the remote origins and the firm continuity of this celebrated form of collective identity. Expanded time and national space are interlaced and both materialize in that peculiar 'production of places' (mausoleums, monuments, signs of events or heroes), which present themselves as institutions of a collective memory, as identity traits which again repudiate the claimed uniformity of the territory.

\section{b) Beyond the nation-State}

On one side, the Newtonian idea of space is the notion nineteenth-century jurists presuppose when they outline their theory of the State; on the other side, the spatial dimension is a decisive component of the nation and a vehicle of its identity pathos. The point of arrival these different, but converging nineteenth-century paths is the building of the legal theory and of the political ideology of the nation-State.

The relationship between the political imagination and the spatial dimension, during the modern era, does not end with the development of the theory of the nation-State. Further considerations arise, sharing the need to go beyond the frame of the simple and necessary connection between State and territory.

Let us consider a relevant legal discipline: international law. It presupposes the peculiarly modern views of State and space, but at the same time, it is obliged to confront problems and tensions from which students of the 'internal' side of sovereignty are exempted. As relevant and recent works

74 I have made use of some passages of a previous essay: Costa (2003). La politica e gli spazi is the title of the first of four seminars, organized by Bruna Consarelli, which have been devoted to the relationship between space and politics: Consarelli (2003a), (2003b) e (2004). A fifth seminar was organized by Lea Campos Boralevi e Sara Lagi: Campos Boralevi / Lagi (2006). 
have highlighted, ${ }^{75}$ international law (in its historical genesis and in its very conceptual frame) is hardly separable from the colonization process and is therefore compelled to allow for a differentiated and heterogeneous spatiality, different from the political geography implied by the ius publicum europaeum.

Then in a later and different phase of international law (mainly in the second half of Twentieth century), 'universalistic' attitudes (stimulated by the increasing emphasis on human rights) combine with 'particularistic' stances (connected with the permanence of national sovereignties), so that the relationship with the spatial dimension becomes more complicated and rougher than ever supposed by nineteenth-century State theories. Such a tension had already smouldered on in eighteenth-century natural law theories and burst forth during the French Revolution: it was the tension between the universalistic dimension of rights and the spatial roots of sovereignty, on which nevertheless the implementation of rights depends.

In the same context in which the State theory develops in tune with a 'Newtonian' view of space, attempts at different combinations between space and politics are not lacking. Indeed, international law was not the only one to feel uncomfortable with the received view of the relationship between politics and space. It was a geographer - Friedrich Ratzel ${ }^{76}$ - who, at the end of nineteenth century, laid the premises of a different approach.

According to Ratzel, territory is not a neutral physical area controlled by a bureaucratic apparatus. His main concern is understanding the vital needs of the human being, in light of the Darwinian theory of evolution. The core of politics is the relationship between a people and their environment, which is not a fixed and inert space, but a dynamic reality on which the life of the people depends. Engaged in the struggle to increase fitness, every people survives if it is able to spill over space and enlarge its vital sphere: "geographic reality forces the historical movement into an uninterrupted transfer to ever new spaces, a continuous migration from a territory to another». ${ }^{77}$ «Space strengthens developing peoples», while "peoples decay when the space at their disposal decreases». It is also the struggle for the vital space,

75 Mannoni (1999); Anghie (2005); Koskenniemi (2002); Nuzzo (2012).

76 Bio-bibliographical data in Köster (2002) $59 \mathrm{ff}$.

77 Ratzel (1899) 158. 
for the «Lebensraum» ${ }^{78}$ (for the «Wohnraum» and for the «Ernährungsraum» ${ }^{79}$ ), the impetus which moves peoples to advance, to challenge the existent boundaries and expand their dominion.

Ratzel moves from an organicistic vision of State, shared by a follower of the same approach (and inventor of the very term of 'geopolitics'): the Swedish geographer and State theorist ${ }^{\mathbf{8 0}}$ Rudolf Kjellén. ${ }^{\mathbf{8 1}}$ The same approach would be followed by the National-Socialist geopolitics of the Thirties and by its prominent spokesperson, Karl Haushofer. ${ }^{82}$ A new discipline geopolitics ${ }^{83}$ - takes shape, which crosses through the Fascist and NationalSocialist ideologies ${ }^{\mathbf{8 4}}$ and arrives, deeply changed, to the present.

A survey of this intellectual path is impossible here. Let me only note that the core of it is, again, a peculiar connection between politics and space. Space and State interlace but their link is rethought on the horizon of the social-Darwinist and imperialistic attitudes increasingly successful in late nineteenth-century Europe. In this context, space was losing its Newtonian evenness and the State was more and more celebrated for its calling to war.

The trailblazer, in this case, had been a geographer, Ratzel, but his idea of State was largely indebted to the legal and political culture of the late nineteenth century. The geographer receives suggestions from the jurist and the jurist treasures the insights of the 'space expert'. The intersection between some peculiar trends of twentieth-century legal theory and the geopolitical discipline would be an issue which could merit further historical examinations.

In this perspective, an emblematic example is Carl Schmitt. His Nomos der Erde and its famous considerations on the relationship between «Ordnung» and «Ortung» are now very popular rhetorical topoi. In our perspective, it is however, worthwhile to recall that the point of origin of Schmitt's theory of

78 RATZel (1901) 5.

79 Ratzel (1901) 56.

80 KJELLÉN (1924) 45: «Die Geopolitik ist die Lehre vom Staat als geographischem Organismus im Raume: also der Staat als Land, Territorium, Gebiet oder, am bezeichnendsten, als Reich».

81 Cf. Holdar (1992).

82 Cf. Ebeling (1994).

83 Among the essays devoted to the history of geopolitics cf. Portinaro (1982); Lorot (1997); Diekmann (2000); Lizza (2001); Losano (2011).

84 On fascist geopolitics cf. Costa (2005) and the relevant contribution of Rodogno (2003). 
the 'big spaces' coincides with the ideal core of geopolitics between the two World Wars: the idea of a space which inflates or shrinks depending on the expansionistic impetus of organic and vital communities. ${ }^{85}$

As the nineteenth-century theory of the State corresponded to the view of a static and homogenous space, so a new vision of politics involves a new idea of space as a dynamic and mobile dimension: a State-centred theory is replaced by the image of a Großraumordnung, of a Reich, which, deeply embedded in a vital and delimited space, has nothing in common with the Anglo-Saxon commercial empire and its universalistic (de-spatialised) stance. $^{86}$

Still again, spatial dimension and political theory strictly interlace. The high esteem in which Schmitt's theory have recently been held, notwithstanding the manifest relationship of his Nomos der Erde with the idea of Großraum, developed by Schmitt during his National-Socialist involvement, depends on the present need to rethink politics on a post-modern (i. e. postState) horizon. The idea of State seems now to be anachronistic and inadequate to understand politics in the new 'global' space, while new (striking but elusive) categories - as the concept of 'empire ${ }^{\mathbf{8 7}}$ - come into play.

c) The 'Orientalist' Space

Political theory in its historical development (from the city to the nation, to the State and to the empire) interlaces with the vision of space. Building political theories is nothing like a simple 'description': the representations of both politics and space are the outcome of 'imagination'. Imagining does not mean, of course, inventing ex nibilo. It instead requires employing the experience data, freely selecting them and, on this basis, outlining conceptual schemes which, on one side, grasp some relevant features of reality but, on the other side, neither exhaust it nor are its simple reflection.

Schmitt's idea of the imperial Großraum is good evidence of the unavoidable one-sidedness of theories. His doctrine aims to offer an extensive interpretation of the global world, but its historical embedment (its 'localization') is more than sufficiently evident: Schmitt's space is the world, but

85 KÖSTER (2002) 203 ff.

86 Schmitt (1939). Cf. Blindow (1999); Pietropaoli (2012) $121 \mathrm{ff}$.

87 Hardt / Negri (2000). On the concept of 'empire' Romanelli (2010). 
inasmuch as it is observed from a 'place' which coincides with the ius publicum europaeum and its last offshoots.

Are different geographical and political visions possible? They are, provided that we are able to move in a new spatial (and temporal) direction: to move not vertically, from the bottom to the top, from the small to the big (from the city to the State and the empire), but horizontally, from one side to another, asking what relationship has been developed between different, but contiguous spaces, between north and south, west and east. If the perspective and the localization of the observer change, different spaces and boundaries can arise.

This is the proposal put forward by Edward Said (a Palestinian author of literary and musicological essays) in his famous work, Orientalism, published in $1978 .^{88}$ Said exhorts us to rethink the relationship between West and East and, in general, between Europe and its 'otherness', the numerous societies with which Europe came in contact along the tragic parabola of colonization.

Space comes again into play: an 'imagined' space, which enables Europe to oppose the West to the East, its own civilization to the 'outer' worlds. The West, when it draws the line which separates it from the East, does not take cognizance of different and autonomous realities, but reduces them to its own standards and converts them into its own shadow. The discourse (which Said calls 'orientalist') does not describe an autonomous reality (the East as an extremely complicated and differentiated world). Europe does not describe, but imagines its 'otherness' as a projection of itself. An 'orientalist' West arises, which spends itself within the discourse that created it. Furthermore, imagining the 'orientalist' East is not a politically innocuous attitude. Instead, the imagined 'orientalist' space is a cultural pattern which, neglecting the otherness and specificity of extra-European civilizations, makes their subjugation easier.

Still again, imagining spaces and drawing boundaries interlace with politics and power. It is in this perspective that Postcolonial studies ${ }^{\mathbf{9 9}}$ try to rethink European history, assuming that Europe's relationship with its 'other-

88 SAÏD (1978). Cf. IsKandar / Rustom (2010).

89 Cf. by way of example Williams / Chrisman (1994); Ashcroft (2007); Young (2001); Lazarus (2004); Mezzadra (2008). 
ness' is not just any event of its history, but an essential component of its identity.

The 'imagined' spaces are also as 'real' as the 'lived' spaces, because both are concretely involved in the dialectics of power and resistance. Indeed, we could refer to further and different ways of conceiving the relationship between politics and space: while the mentioned strategies take already existent political orders into consideration, the link between space and politics can be employed to imagine alternative and future arrangements. Let us think, on one side, about that intricate network of discourses and theories referable to the category of 'cosmopolitism'90 and, on the other side, about the 'literary genre' according to which places are not situated in our spatial and temporal reality, but are imagined as 'exterior' to it, as 'u-topias': as places belonging to radically alternative worlds.

In both cases, we witness an original combination between space and politics, capable of provoking utterly 'real' effects in the social and political dynamics: outlining and proposing 'alternative places' can be a serious and demanding game. It is however, another game, whose rules are different from those brought back to mind by the 'spatial turn'.

\section{Bibliography}

Anghie, Antony (2005), Imperialism, Sovereignty and the Making of International Law, Cambridge: Cambridge University Press (available at: http://dx.doi.org/ 10.1017/CBO9780511614262)

Appadurai, Arjun (1996), Modernity at Large: Cultural Dimensions of Globalization, Minneapolis: University of Minnesota Press

Ardrey, Robert (1966), The Territorial Imperative. A Personal Inquiry into the Animal Origins of Property and Nations, New York: Atheneum

Ashcroft, Bill et al. (2007) (eds.), Post-colonial Studies: The Key Concepts, London: Routledge

Belina, Bernd, Michel Boris (2007) (Hrsg.), Raumproduktionen. Beiträge der Radical Geography. Eine Zwischenbilanz, Münster: Westfälisches Dampfboot

Blanco, Luigi (a cura di) (2008), Organizzazione del potere e territorio. Contributi per una lettura storica della spazialità, Milano: Angeli

90 An excellent history of cosmopolitism in ScuccimarRa (2006). 
Blaut, James Morris (1993), The Colonizer's Model of the World. Geographical Diffusionism and Eurocentric History, New York: Guilford Press

Blindow, Felix (1999), Carl Schmitts Reichsordnung. Strategie für einen europäischen Großraum, Berlin: Akademie Verlag

Brenner, Neil et al. (2003) (eds.), State/Space. A Reader, Malden: Blackwell

Campos Boralevi, Lea, Sara Lagi (a cura di) (2009), Viaggio E Politica (V Giornata Di Studio "Figure Dello Spazio, Politica E Società"), Firenze: Firenze University Press

Carle, Lucia (2013), Dinamiche identitarie. Antropologia storica e territori, Firenze: Firenze University Press

Castree, Noel (2009), The Spatio-temporality of Capitalism, in: Time \& Society 18,1, 26-61 (available at: http://dx.doi.org/10.1177/0961463X08099942)

Chittolini, Giongio (1994), Organizzazione territoriale e distretti urbani nell'Italia del tardo Medioevo, in: Chittolini, Giorgio, Dieter Willoweit (a cura di), L'organizzazione del territorio in Italia e Germania: secoli XIII-XIV, Bologna: Il Mulino, 7-26

Cомba, Rinaldo (1981), Il territorio come spazio vissuto. Ricerche geografiche e storiche sulla genesi di un tema di storia sociale, in: Società e storia IV, 11, $1-27$

Consarelli, Bruna (a cura di) (2003a), Figure dello spazio, politica e società, 2. Metafore dello spazio, Firenze: Firenze University Press

Consarelli, Bruna (a cura di) (2003b), Figure dello spazio, politica e società, 3. Gli spazi immaginati, Firenze: Firenze University Press

Consarelli, Bruna (a cura di) (2004), Figure dello spazio, politica e società, 4. Spazi e politica nella modernità tecnologica, Firenze: Firenze University Press

Cosgrove, Denis (2004), Landscape and Landschaft, in: German Historical Institute Bulletin 35, 57-71

Costa, Pietro (2003), La civitas e il suo spazio: la costruzione simbolica del territorio fra medio evo ed età moderna, in: Consarelli, Bruna (a cura di), Figure dello spazio, politica e società, 1. La politica e gli spazi, Firenze: Firenze University Press, 43-57

Costa, Pietro (2005), Il fardello della civilizzazione. Metamorfosi della sovranità nella giuscolonialistica italiana, in: Quaderni Fiorentini 33-34, 169-257

Costa, Pietro (2012), Nazione, diritti, Stato, in: Aláez Corral, Benito (ed.), El pueblo del estado. Nacionalidad y ciudadanía en el estado constitucionaldemocrático, Oviedo: Junta General del Principado de Asturias, 31-84

Costa, Pietro (2012), Review of Angelo Torre, Luoghi, in: Quaderni Fiorentini 41, $808-818$

Crampton, Jeremy, Stuart Elden (2007) (eds.), Space, Knowledge and Power: Foucault and Geography, Aldershot: Ashgate

De Certeau, Michel, (1984), The Practice of Everyday Life, Berkeley: University of California Press 
De Vergottini, Giovanni (1977), Origini e sviluppo storico della comitatinanza, in: De Vergottini, Giovanni, Scritti di Storia del Diritto Italiano, Milano: Giuffrè, 3-122

Di Méo, Guy, Pascal Buléon (2005), L’espace social. Une lecture géographique des sociétés, Paris: Armand Colin

Diekmann, Irene et al. (2000) (Hrsg.), Geopolitik. Grenzgänge im Zeitgeist, Band 1.1, Potsdam: Verlag für Berlin-Brandenburg

Driver, Felix, Raphael Samuel (1995), Rethinking the Idea of Place, in: History Workshop Journal 39, 5-7 (available at: http://dx.doi.org/10.1093/hwj/39.1.1-t)

Durкheim, Émile (1912), Les formes élémentaires de la vie religieuse. Le système totémique en Australie, Paris: Librairie F. Alcan

Durkheim, Émile, Marcel Mauss (1903), De quelques forme primitives de classification. Contribution à l'étude des représentations collective, in: L'Année sociologique $6,1-72$

Ebeling, Frank (1994), Geopolitik: Karl Haushofer und seine Raumwissenschaft 1919-1945, Berlin: Akademie Verlag

Elias, Norbert (1992), An Essay on Time (The Collected Works of Norbert Elias, vol. 9), Dublin: University College Dublin Press

Ethington, Philip J. (1997), The Intellectual Construction of "Social Distance": Toward a Recovery of Georg Simmel's Social Geometry, in: Cybergeo: European Journal of Geography (available at: http://cybergeo.revues.org/227)

Ethington, Philip J. (2007), Placing the Past: 'Groundwork' for a Spatial Theory of History, in: Rethinking History 11, 4, 465-493

Foucault, Michel (1994), Des espaces autres, in: Foucault, Michel, Dits et écrits, vol. IV, Paris: Gallimard, 752-762

Gabba, Emilio, Aldo Schiavone (1999) (a cura di), Polis e piccolo Stato tra riflessione antica e pensiero moderno, Como: New press

Giddens, Anthony (1987), Social Theory and Modern Sociology, Cambridge: Polity Press

Glauser, Andrea (2006), Pionierarbeit mit paradoxen Folgen? Zur neueren Rezeption der Raumsoziologie von Georg Simmel, in: Zeitschrift für Soziologie 35, 4, 250-268

Grossi, Paolo (1968), Le situazioni reali nell'esperienza giuridica medievale. Corso di Storia del diritto, Padova: CEDAM

Hardt, Michael, Antonio Negri (2000), Empire, Cambridge (Mass.): Harvard University Press

Harley, John Brian (1988), Silences and Secrecy: The Hidden Agenda of Cartography in Early Modern Europe, in: Imago mundi 40, 57-76

Harvey, David (1973), Social Justice and the City, London: E. Arnold

Harvey, David (2006), Spaces of Global Capitalism, London: Verso

Hespanha, António Manuel (1982), L'espace politique dans l'Ancien régime, in: Boletim da Faculdade de Direito, Universidade de Coimbra (Estudos em homenagem aos Profs. Manuel Paulo Merêa e Guilherme Braga da Cruz) LVIII, 455-510 
Holdar, Sven (1992), The ideal State and the power of geography. The life-work of Rudolph Kjellén, in: Political Geography Quarterly XI, 3, 307-324

Iskandar, Adel, Hakem Rustom (2010) (eds.), Edward Saïd: A Legacy of Emancipation and Representation, Berkeley: University of California Press

Kern, Stephen (1983), The Culture of Time and Space 1880-1918, Cambridge (Mass.)

KJellén, Rudolf (1924), Der Staat als Lebensform, Berlin: Harvard University Press

Koselleck, Reinhart (1979), Vergangene Zukunft. Zur Semantik geschichtlicher Zeiten, Frankfurt am Main: Suhrkamp

Koskenniemi, Martti (2002), The Gentle Civilizer of Nations: The Rise and Fall of International Law, 1870-1960, Cambridge: Cambridge University Press

Köster, Werner (2002), Die Rede über den 'Raum'. Zur semantischen Karriere eines deutschen Konzepts, Heidelberg: Synchron

Lallement, Michel (2008), Une antinomie durkheimienne [...] et au-delà, in: Temporalités $8,1-14$ (available at: http://temporalites.revues.org/72)

Latini, Carlotta (2002), Il privilegio dell'immunità. Diritto d'asilo e giurisdizione nell'ordine giuridico dell'età moderna, Milano: Giuffrè

Lazarus, Neil (ed.) (2004), The Cambridge Companion to Postcolonial Literary Studies, Cambridge: Cambridge University Press (available at: http:// dx.doi.org/10.1017/CCOL0521826942)

Leccardi, Carmen (2009), Sociologie del tempo. Soggetti e tempo nella società dell'accelerazione, Roma-Bari: Laterza

Lefebvre, Henri (1974), La production de l'espace, Paris: Anthropos

Leibniz, Gottfried Wilhelm (1717), Mr. Leibnitz's Third Paper, being An Answer to Dr. Clarke's Second Reply, in: Clarke, Samuel, A Collection of Papers, Which passed between the late Learned Mr. Leibnitz, and Dr. Clarke, In the Years 1715 and 1716, $\$ 4$ London: Knapton (available at:

http://www.newtonproject.sussex.ac.uk/prism.php?id=1)

Lizza, Gianfranco (2001), Geopolitica. Itinerari del potere, Torino: UTET

Lorot, Pascal (1997), Storia della geopolitica, Trieste: Asterios

Losano, Mario G. (2011), La geopolitica del Novecento. Dai grandi spazi delle dittature alla decolonizzazione, Milano: Mondadori

Mannoni, Stefano (1999), Potenza e ragione: la scienza del diritto internazionale nella crisi dell'equilibrio europeo, 1870-1914, Milano: Giuffrè

Mannori, Luca (2008), La nozione di territorio fra antico e nuovo regime. Qualche appunto per uno studio sui modelli tipologici, in: Cammelli, Marco (a cura di), Territorialità e delocalizzazione nel governo locale, Bologna: Il Mulino, 43-63

Marchetti, Paolo (2001), De iure finium. Diritto e confini tra tardo medioevo ed età moderna, Milano: Giuffrè

Marramao, Giacomo (2005), Minima temporalia: tempo, spazio esperienza, Roma: L. Sossella

Marramao, Giacomo (2013), Spatial turn: spazio vissuto e segni dei tempi, in: Quadranti. Rivista Internazionale di Filosofia Contemporanea 1, 31-36 
Massey, Doreen (1995), Places and Their Pasts, in: History Workshop Journal 39, 182-92

Massey, Doreen (2005), For Space, Los Angeles: SAGE

Mezzadra, Sandro (2008), La condizione postcoloniale. Storia e politica nel presente globale, Verona: Ombre corte

Minca, Claudio, Luiza Bialasiewicz (2004), Spazio e politica. Riflessioni di geografia critica, Padova: CEDAM

Murphy, Alexander B. (2012), Entente territorial: Sack and Raffestin on territoriality, in: Environment and Planning D: Society and Space 30, 159-172

Nuzzo, Luigr (2012), Origini di una scienza: diritto internazionale e colonialismo nel 19. secolo, Frankfurt am Main: V. Klostermann

Pacelli, Donatella (2007), Introduzione. La cornice spazio-tempo: verso una riconcettualizzazione del contesto sociale, in: Pacelli, Donatella, Maria Cristina Marchetti (a cura di), Tempo, spazio e società. La ridefinizione dell'esperienza collettiva, Milano: F. Angeli, 9-23

Pastore, Alessandro (a cura di) (2007), Confini e frontiere nell'età moderna. Un confronto fra discipline, Milano: F. Angeli

Petti Balbi, Giovanna (a cura di) (2001), Comunità forestiere e “nationes” nell'Europa dei secoli XIII-XVI, Napoli: Liguori

Pietropaoli, Stefano (2012), Schmitt, Roma: Carocci

Portinaro, Pier Paolo (1982), Nel tramonto dell'Occidente: la geopolitica, in: Comunità XXXVI, 184, 1-42

Raffestin, Claude (1980), Pour une géographie du pouvoir, Paris: Librairies Techniques

Raffestin, Claude (2007), Could Foucault have revolutionized geography?, in: Crampton, Jeremy, Stuart Elden (2007) 129-137

Ratzel, Friedrich (1899), Anthropogeographie. Grundzüge der Anwendung der Erdkunde auf die Geschichte, Stuttgart: J. Engelhorn

Ratzel, Friedrich (1901), Der Lebensraum. Eine biogeographische Studie, Tübingen: Lauppschen Buchhandlung

Rodogno, Davide (2003), Il nuovo ordine mediterraneo, Torino: Bollati Boringhieri

Romanelli Raffaele (a cura di) (2010), Impero, imperi, Napoli: L'ancora del Mediterraneo

Rosa, Hartmut (2005), Beschleunigung. Die Veränderung der Zeitstrukturen in der Moderne, Frankfurt am Main: Suhrkamp

Rosenwein, Barbara (1999), Negotiating Space: Power, Restraint, and Privileges of Immunity in Early Medieval Europe, Ithaca NY: Cornell University Press

Ruschi, Filippo (2012), Questioni di spazio. La terra, il mare, il diritto secondo Carl Schmitt, Torino: Giappichelli

SAck, Robert D. (1983), Human Territoriality: A Theory, in: Annals of the Association of American Geographers 73, 1, 55-74

SaÏD, Edward W. (1978), Orientalism, New York: Pantheon Books

Salvemini, Biagio (2006), Il territorio sghembo. Forme e dinamiche degli spazi umani in età moderna. Sondaggi e letture, Bari: Edipuglia 
Schlögel, Karl (2003), Im Raume lesen wir die Zeit. Über Zivilisationsgeschichte und Geopolitik, München: Carl Hanser Verlag

Schmaus, Warren (2004), Rethinking Durkheim and His Tradition, Cambridge: Cambridge University Press (available at: http://dx.doi.org/10.1017/ CBO9780511498329)

Schmitt, CARL (1939), Völkerrechtliche Großraumordnung mit Interventionsverbot für raumfremde Mächte. Ein Beitrag zum Reichsbegriff im Völkerrecht, Berlin: Deutscher Rechtsverlag

Scuccimarra, Luca (2006), I confini del mondo: storia del cosmopolitismo dall'antichità al Settecento, Bologna: Il Mulino

Sereni, Emilio (1961), Storia del paesaggio agrario italiano, Bari: Laterza

Simmel, Georg (1903), Soziologie des Raumes, in: Jahrbuch für Gesetzgebung, Verwaltung und Volkswirtschaft im Deutschen Reich 27, 1, 27-71

Soja, Edward W. (1989), Postmodern Geographies. The Reassertion of Space in Critical Social Theory, London: Verso

Somaini, Francesco (2012), Territory, territorialisation, territoriality: Problems of definition and historical interpretation, in: Plurimondi V, 10, 19-47

Spicciani, Amleto, Cinzio Violante (1997-1998) (a cura di), La signoria rurale nel medioevo italiano, Pisa: Edizioni ETS

Sturani, Maria Luisa (2008), Le rappresentazioni cartografiche nella costruzione di identità territoriali: materiali e spunti di riflessione dalla prospettiva della storia della cartografia, in: Blanco, Luigi (a cura di) (2008) 189-213

Sue, Roger (1994), Temps et ordre social. Sociologie des temps sociaux, Paris: Presses universitaires de France

Tabboni, Simonetta (2001), The Idea of Social Time in Norbert Elias, in: Time \& Society 10, 1, 5-37 (available at: http://dx.doi.org/10.1177/0961463X01010001001)

TAylor, Peter (1994), The state as container: territoriality in the modern worldsystem, in: Progress in Human geography XVII, 151-62 (available at: http:// dx.doi.org/10.1177/030913259401800202)

Torre, Angelo (2011), Luoghi. La produzione di località in età moderna e contemporanea, Roma: Donzelli

Turco, Angelo (2010), Configurazioni della territorialità, Milano: Angeli

Vaccari, Pietro (1920), La territorialità come base dell'ordinamento giuridico del contado (Italia Superiore e media), in: Bollettino della Società Pavese di Storia Patria XX, 195-233

Vallejo, Jesús (1992), Ruda equidad, ley consumada. Concepción de la potestad normativa (1250-1350), Madrid: Centro de estudios constitucionales

Van Houtum, Henk et al. (2005) (eds.), Bordering Space, Aldershot: Ashgate

Virilio, Paul (1977), Vitesse et politique. Essai de dromologie, Paris: Galilée

Wanklyn, Harriet (1961), Friedrich Ratzel: A Biographical Memoir and Bibliography, Cambridge: Cambridge University Press

Williams, Patrick, Laura Chrisman (1994) (eds.), Colonial Discourse and Postcolonial Theory. A Reader, New York: Columbia University Press 
Withers, Charles W. J. (2009), Place and the "Spatial Turn" in Geography and in History, in: Journal of the History of Ideas 70, 4, 636-658

Young, Robert J.C. (2001), Postcolonialism. An Historical Introduction, Oxford: Blackwell

Zumthor, Paul (1993), La Mesure du monde, Paris: Editions du Seuil 


\section{Sobre el "Espacio" y el "Tiempo" y el "Estado de las Personas”. Una mirada desde la Historia del Derecho}

\section{Introducción}

Para el jurista, dogmático o histórico, los textos con que trabaja, aquellos que lee y relee, son fuente inexhausta de dudas y sorpresas. Que bien sabemos cuánto dicen, pero también, cuánto ocultan las palabras, y las relaciones que se establecen entre ellas y aquello que designan o pretenden designar. Sabemos, igualmente, aunque muchas veces no lo tengamos tan presente, cuán complejas son las relaciones que como lectores instauramos entre aquellas palabras de los textos y la herencia cultural de que somos portadores. En fin, sabemos, cómo concurre nuestro uso de las palabras a clasificar el mundo, y a crearlo para nosotros mismos, si bien sólo podemos intuir el modo en que estas operaciones pueden proyectarse sobre aquel "otro" que nos lee o leerá algún día.

En las líneas que siguen me limitaré a sugerir algunas de las dudas y sorpresas que pueden alzarse cuando se realiza el intento de aproximarse a unos textos que han desempeñado un singular papel en la historia del lugar que han podido ocupar el "espacio" y el "tiempo" en el concreto campo del "estado de las personas". Se trata, de unos textos que, como en la mayor parte de la historia de nuestra cultura jurídica, los juristas hallaban en el Corpus Iuris Civilis.

En los textos del Corpus Iuris Civilis los juristas del derecho común se encontraban con las voces status y persona u homo en múltiples pasajes, pero singularmente en un título del Digesto $(1,5)$ y en dos de las Instituciones (I, 3 y I, 16).

En el citado título del Digesto $(1,5)$ y en el primero de los mencionados de las Instituciones (I, 3), se recibían, con mayor o menor extensión, algunos pasajes del libro primero de las Instituciones de Gayo. De ellos resultaba una "triple" división del derecho de las personas (de iure personarum): la summa divisio: liberi aut servi; a la que seguía otra división: sui iuris o alieni iuris; y 
una última: quae in tutela, quae in curatela o ceteras personas, quae neutro iure tenentur. En términos generales, y en sólo uno de los puntos que aquí interesan, estos textos en la cultura del derecho común tendieron a leerse con el matiz de destacarse que en ellos se consagraban unas "divisiones de las personas" o "de los hombres", más que una división del "derecho de las personas". ${ }^{1}$

Por otra parte, los textos del otro título de las Instituciones (I, 16) se ocupaban de la capitis deminutio, concebida como prioris status commutatio, lo que podía acontecer por tres modos diversos (tribus modis accidit). La lectura de esos pasajes condujo a que tempranamente en la cultura del derecho común se consolidara como una idea básica la de la existencia de tres status de las personas: civitatis, libertatis, familiae. Una trilogía que, aunque no propia de la jurisprudencia romana sí construida sobre la lectura de algunos de sus textos, gozó de singular fortuna y dio origen a más de una exposición sigular sobre ella. ${ }^{2}$

En aquellos dos campos operativos, el de la división de derecho de las personas y el de la capitis deminutio, el spatium tenía un lugar, aunque no de un modo propio y directo. La tradición ordenadora de las Institutiones de Gayo había situado el tratamiento de los cives, latini, peregrini y hostes a propósito de la summa divisio de las personas, es decir, la de libres o esclavos, aunque en la compilación justinianea muchas de las cuestiones a que ellas habían dado lugar o habían desaparecido o se mostraban desdibujadas, como consecuencia de la pérdida de la centralidad que experimentó la noción de

1 Esta lectura ya aparecía como consolidada en la glossa ordinaria, como resultaba de la misma divisio del texto inicial del título III de las Institutiones: Accursius (1559) gl. 'Sum$m a$, fol. 30: «Totus iste tit.[ulum] dividitur in sex partes. Nam primo ponit divisiones personarum [...]». En muchos casos tal divisio legis fue recibida literalmente en obras posteriores, $v$. gr. Gambiglioni de Aretio (1574) fol. 20v: «Totus iste tit.[ulum] dividitur in sex partes. Nam primo ponit divisiones personarum [...]». En otros, el comentario o explicación del dicho título partía con frases que destacaban que en él se trataba de la “división de las personas", v. gr. Ubaldi (1586) fol. 8: «Prima divisio personarum est, quod quidam liberi, quidam, servi sunt». Lo mismo puede decirse de la explicación del título correspondiente del Digesto $(1,5,3), v$. gr. Saxoferrato (1538) fol. 41v: «Summa. Primo ponit divisio personarum $[\ldots]$ ».

2 En la glossa ordinaria ya podía hallarse una lectura que asentaba la idea de la existencia de tres “estados de las personas”, Accursius (1559) gl. 'Status', fol. 101: «Hic status consistit in tribus: in libertate, civitate, \& familia [...]»; vide BicQuilley (1737). 
cives a partir del siglo III d. C. Con todo el spatium no había sido el factor determinante de la categoría de cives, de ahí que su papel en la determinación del estado de los hombres o de las personas no hubiera operado directamente, sino de un modo accidental, en cuanto que a propósito de él se habían planteado ciertas cuestiones "espaciales", como la del lugar donde se había nacido o el origen.

Durante el siglo XVI y buena parte del XVII no se apreciaban mayores innovaciones en el tratamiento que los juristas hacían de aquellos pasajes del Corpus Iuris Civilis, aunque ellos sí generaron una amplia y sostenida discusión acerca de las nociones de status, caput y capitis deminutio. En el curso de esa discusión hubo una especial preocupación por determinar qué había de entenderse por status y, sin perjuicio de los naturales matices que podían observarse entre unos juristas y otros, se asentó la idea de que, en esta sede, aquella voz significaba la "condición" de las personas en orden a la libertad, la ciudadanía, o la familia. ${ }^{3}$

Hubo, sin embargo, desde el siglo XVI y en el genérico ambiente cultural que solemos llamar humanismo y en el ámbito del usus modernus Pandectarum, algunas lecturas del status que introdujeron unos elementos nuevos, que abrían la reflexión sobre él a campos operativos mucho más amplios. Aquí interesa destacar sólo dos de ellas:

i) la que se realizaba con ciertas categorías y nociones que procedían del universo de la filosofía; y

3 Hotman (1569) fol. 66, ad Inst. 1, 16, 1: «Hoc igitur primum intelligi oportet, status verbo in hoc tratatu significari a Iurisconsultis, conditionem personae in eorum ordine, qui vel libertatem, vel cum libertate civitatem, vel cum utraque, familiam obtinent»; Harprecht (1562) ad Inst. 1, 16, 1, fol. 107: «Per statum autem non quamvis hominis Conditionem (ut alias communi loquendi usu hoc verbum status accipi solet) sed eam, quae in Libertate, Civitate \& familia consistit [...]»; Pichardo de Vinuesa (1630) ad Inst. 1, 16, n. 2, fol. 83: «Unde cum omnia haec capita perimuntur, libertatis, civitatis, au familiae, vel alterum ex his, capite minus dicimus. Igitur, status, in definitione conditionem significat, \& ius quod aliquis ob eam causam habet, quia caput sive locum in ordine aliquo illorum trium obtinet, ut probat hic Hotom. in pr.»; PÉrez (1639), ad Inst. 1, 3, fol. 11: «Status sive conditio hominum, secundum quam in hac civili societate regitur, quaeque in tribus principaliter consistere dicitur, Libertate, Civitate \& Familia: nam de personis traditur, cuius status ac conditionis sit unusquisque; liber, ac servus; ingenuus, ac libertinus; sui ac alieni iuris». 
ii) aquellas otras que introducían distinciones novedosas, derivadas normalmente de una singular preocupación por la relación que existía entre las categorías de homo y persona, y por el modo en que ellas se presentaban en su tiempo.

\section{$\left.1^{\circ}\right)$ El status y el recurso al lenguaje y categorías de la filosofía}

Entre las primeras lecturas que pueden situarse en esta perspectiva novedosa se cuenta la de Ulric Zasius (1461-1536) que, por una parte, calificaba al status como una "cualidad habitual" (babitualis qualitas) y, por otra, no la defínía por la textual trilogía libertas, civitas, familia, sino por un cierto ser: "para ser" (ut esse) libre, esclavo, noble, legítimo, etc. ${ }^{4}$ Muestra también de su lectura desde categorías propias de la filosofía era su explicación de quiénes eran statuliberi: aquellos que estaban in habitu de libertad, aunque in actu fueran esclavos. ${ }^{5}$ Esta línea se mantuvo en los discípulos de Zasius, y, así, por ejemplo, Joachim Mysinger von Frundeck (1514-1588) escribía que el status no era más que una cualidad y condición (qualitas et conditio) de la persona para ser (ut esse) libre o ingenuo, o noble, o esclavo. ${ }^{6}$

En una perspectiva similar, Hugo Donellus (1527-1591) se preocupaba de esclarecer la relación que existía entre status y ius. La explicaba desde las categorías de causa y effectus. Para Donellus, status era la condición de cada persona, y ius la facultad de vivir y de hacer lo que placiera, atribuida por aquella condición. De ello resultaba que el estado y condición fuera la causa, y el derecho de la persona el efecto de aquel estado y condición. ${ }^{7}$ Esta misma línea fue continuada por Arnoldo Vinnius (1588-1657), para quien el derecho de la persona era aquel que seguía al estado y condición de ésta, pues el mismo status no era más que la condición o cualidad de la persona que hacía

4 Zasius (1539) ad Dig. 5, 1, 5, fol. 10: «Status nihil aliud est quam conditio \& (ut ita dixerim) habitualis qualitas, ut, esse liberum, esse nobilem, esse legitimum \&c.».

5 Zasius (1539) ad Dig. 5, 1, 5, fol. 10: «A statu veniunt statuliberi, qui sunt in habitu libertatis, licet actu servi sunt [...]».

6 Mynsiger a Frundeck (1595) ad Inst. 1, 3, 1, n. 7, fol. 24: «Est enim status, nihil aliud quam personae qualitas \& conditio: ut esse liberum vel ingenuum, sive nobilem, esse servum».

7 Donnellus (1840) lib II, c. IX, II, col. 241: «Status est conditio personae cujusque. Jus, facultas vivendi, et faciendi, quae velis, quae ei conditioni tribuitur. Itaque, ut dixi, status, et conditio causa est: jus personae, illius status et conditionis effectus [...]». 
que usara de este o aquel derecho, como ser libre, siervo, ingenuo, liberto, alieni iuris, sui iuris, de modo que el status operaba como causa y el derecho como efecto. $^{8}$

\section{$\left.2^{\circ}\right) \quad$ Unas novedosas distinciones del status}

En el curso del siglo XVII ciertos juristas se movieron con una mayor libertad en el momento de enfrentar la lectura de los textos que tocaban al status de las personas. Esa mayor libertad, en principio, pareciera que estuvo movida por el propósito de ampliar el campo operativo de la categoría de status a las diversas realidades de su tiempo. Ello condujo a que, por una parte, aparecieran unos nuevos criterios de distinción aplicados al status y, por otra, a que resultaran atraídas a esta sede algunas cuestiones que en los textos justinianeos se situaban en otros lugares, en especial, las que tocaban a las personas que estaban por nacer (nasciturus) y a aquellas que gozaban de imperium.

i) Hacia el status publicus y status privatus de las personas Una de tales lecturas novedosas, interesante entre otros aspectos por la proyección de la categoría de status al campo de la iurisdictio y el imperium, era la que hacía Hermann Vulteius (1555-1634) en su Commentarius a las Instituciones justinianeas.

Su punto de partida se situaba en la relación que existía entre bomo y persona: pues la primera era un vocablo de la naturaleza, y la segunda lo era del derecho civil, pues persona no era más que el hombre que tenía caput civile. ${ }^{9}$ Entre los aspectos originales de esta lectura se hallaba el que se mostrara como especialmente atenta a la realidad de su tiempo, y ello hacía que se moviera con una mayor libertad de cara a los textos romanos y a sus categorías. De ahí que afirmara que en el derecho germano la consideración de los hombres era triple: una por razón de sexo, otra por razón de dignidad,

8 Vinnius (1665) ad Inst. 1, 3, fol. 23: «Item jus personae hic esse, quod statum \&conditionem personae sequitur: nam status ipse est personae conditio, aut qualitas, quae efficit, ut hoc vel illo jure utatur; ut esse liberum, esse servum, esse ingenuum, esse libertinum, esse alieni, esse sui juris. Itaque status rationem causae, jus effecti habet».

9 Vulteius (1613) ad Inst. 1, 3, fol. 40: «Persona est homo habens caput civile [...] Homo vocabulum est naturae; persona, juris civilis». 
y otra por razón de status. ${ }^{10}$ Superaba, así, la asentada trilogía libertas, civitas, familia, y afincaba otra nueva: ex sexu, ex dignitate, ex statu. De ella se derivarían, entre otras, dos importantes consecuencias: desplazaba la distinción entre cives y peregrinus, desde el status a la dignitas, y estrechaba el campo operativo de la categoría de status. En cuanto a la primera, ex dignitate, los hombres podían ser "públicos" o "privados". Públicos eran los que tenían imperium, como el príncipe y magistrados, o cargas u oficios, como los ministros de los príncipes y magistrados. De los "privados", unos lo eran por razon de orden (ratione ordinis), y otros por su modo de vida (ex vitae instituto). Así, por razón de orden, unos eran peregrinos y otros ciudadanos, y estos o nobles o plebeyos; y por razón de su modo de vida cada uno elegía su condición de vida. ${ }^{\mathbf{1 1}}$ En cuanto a la segunda, la noción de status quedaba circunscrita a las distinciones entre libres y esclavos, sui iuris y alieni iuris, y sujetos a tutela o curatela, o no sujetos a ninguna de ella. ${ }^{\mathbf{1 2}}$

\section{ii) El status naturalis y el status civilis de las personas}

Otra lectura novedosa, que redefinía las distinciones del status y los criterios a que ellas atendían, fue la que instauró la diferenciación básica entre status naturalis y status civilis. En términos generales esta distinción del status se consolidó en dos espacios metodológicos diversos, a saber: en el del usus modernus Pandectarum, como ya podía leerse clara y ampliamente desarrollada en las obras de Georg Adam Struve (1619-1692), en particular en sus Syntagma jurisprudentiae (1658), y en el más vinculado a la tradición del

10 Vulteius (1613), fol. 41: «Hominum in jure nostro consideratio tripertita est, una ex sexu, altera dignitate, \& tertia ex statu».

11 Vulteius (1613), fol. 41: «Ex dignitate hominum alii publici sunt, alii privati. Publici habent vel imperium, ut princeps \& magistratus, vel munus sive officium, ut principis $\&$ magistratuum ministri, de quibus multa in duodecimo libro Codicis. Privati alii sunt ratione ordinis, alii vitae suae instituto. Ratione ordinis alii peregrini sunt, alii cives: $\&$ rursus civium alii nobiles sunt, alii plebeji. Ex vitae instituto, prout sibi quisque vivendi conditionem elegit».

12 Vulteius (1613), fol. 41: «Tertia, quae ex statu \& conditione hominis sumitur, secundum Justinianeam methodum huius loci est propria. Ex hac hominum alii sunt servi, alii liberi iterum ingenui vel libertini: \& hic utique rursus juris sunt sui vel alieni: \& qui juris sunt alieni, sunt in potestate sive jure Dominico, vel ut potestate sive jure patrio: qui autem juris sui sunt, sunt vel in tutela, vel in cura, vel in neutra». 
humanismo, cuya expresión más representativa se hallaba en los trabajos de Jean Domat (1625-1696), en especial en su Les lois civiles (1689).

\section{a) En el espacio del usus modernus Pandectarum}

'Persona', era un voz que para Struve significaba al hombre que vivía en sociedad civil, pues todo hombre tenía atribuido algún estado o condición, o por la misma naturaleza, o por el derecho. ${ }^{13} \mathrm{Al}$ primero de ellos lo llamaba Status naturalis, y según él los hombres se distinguían primero por razón de sexo (ratione sexus), ${ }^{\mathbf{1 4}}$ y luego por razón de nacimiento (ratione nativitatis), de modo que uno era el que estaba por nacer (nasciturus) y otro el ya nacido (natus). ${ }^{15}$ Al segundo lo llamaba Status civilis, a propósito del que aclaraba que el derecho, en virtud del cual el hombre estaba constituido en una cierta condición, se decía jus Personarum, y en especie Satus hominum. ${ }^{\mathbf{1 6}}$ Status aquí era, pues, la condición de los hombres, política o civil, que hacía que usara en la sociedad civil de un derecho u otro. ${ }^{17}$ Tal condición, o status, era o absoluta, según la cual los hombres se diferenciaban en libres y esclavos, o relativa, y esta podía serlo en relación con la familia, según la cual se distinguían en sui iuris y alieni iuris, o en relación con una ciudad determinada, conforme a la cual las personas se diferenciaban en peregrinos y ciudadanos. ${ }^{18}$ Los orígenes de la distinción entre status naturalis y status civilis se

13 Struve (1692): «Exercitatio tertia [...] De Statu Hominum», n. II, fol. 51: «Persona in genere significat hominem in societate civili viventem [...] Habet vero homo aliquem statum sive conditionem attributam, vel ab ipsa natura [...] vel a jure».

14 StRUve (1692) n. II, fol. 51: «Juxta statum naturalem distinguuntur homines primo ratione sexus $[\ldots] »$.

15 Struve (1692) n. III, fol. 52: «Deinde ratione nativitatis alius nasciturus f. in utero est, alius natus».

16 Struve (1692) n. V, fol. 53: «Jus, quo in certa conditione homo esta constitutus; dicitur jus Personarum $\&$ in specie Status hominum».

17 Struve (1692) n. VI, fol. 53: «Status hic est, conditio hominum politica seu civilis, quae facit, ut hoc vel illo Jure quis in societate civile utatur».

18 Struve (1692) n. VII, fol. 53-54: "Conditio ejusmodi vel est absoluta vel relata: absoluta est ea, cum distinguuntur homines in liberos \&servos, quae propterea summa dicitur divisio 1. 3. h. r. pr. de I. p. Relata respicit aut familiam, aut civitatem cxertam. Juxta priorem relationem alia divisione distinguuntur homines, quod vel sui juris sint, vel alieno juri subjecti: Alieno juri subjecti sunt porro aut in potestate dominorum, aut parentum $[\ldots]$ Juxta posteriorem relationem personae distinguuntur in peregrinos $\&$ cives $[\ldots]$.. 
remontaban a la lectura de Matthaeus Wesenbeck (1531-1586). Su punto de partida se hallaba, precisamente, en que Wesenbeck leía el título III de las Instituciones justinianeas (De iure personarum) desde la siguiente premisa: la summa divisio personarum con la que se abría este título (liberi aut servi) era una división de derecho (iuris divisio), asumida del derecho de gentes, y no una división según la naturaleza (ex natura), porque, de acuerdo con esta, la prima y summa divisio era la que diferenciaba entre varones y hembras. ${ }^{19}$ Desde esta misma premisa leía el título De statu hominum del Digesto (1, 5), y ello permitía establecer una triple división de las personas: una natural, otra del derecho de gentes, y otra del derecho civil. ${ }^{20}$ Esta lectura de Wesenbeck fue asumida y desarrollada por Heinrich Hahn (1605-1668), de quien fue discípulo el ya citado Struve. En efecto, Hahn en sus observaciones a los Comentarios al Digesto de Wesenbeck escribía que una de las dos acepciones en la que los juristas admitían la palabra status era la de alguna condición o cualidad, y que ella podía aplicarse: a) lata y generalmente, como cuando se hablaba del estado de la república o el estado público y privado o; b) referida al hombre. En este último sentido, ella admitía un triple uso: 1) generalmente denotaba cualquier condición natural del hombre, es decir, aquellas que tenía por naturaleza, como la que había por razón de sexo entre varones y hembras, o entre ya nacidos y aún en el vientre materno; 2o) más estricta y especialmente se refería "al estado civil de los hombres" (ad statum hominum civilem) o político; y 3o) estrictísimamente se tomaba pro parte status, como cuando se lo tomaba por "libertad" o por "ciudadanía"

19 Wesenbeck (1585b) ad Inst. 1, 3, 1, n. 1, fol. 12: «Prima] Prima \& ultima, qua reliquae omnes continetur, quod quidem ad ius $\&$ statum attinet, qua de re hic quaeritur: nam si naturam spectes, summa divisio \&prima erit, qua natura omnes homines in mares \& foeminae discrevit».

20 Wesenbeck (1585a) ad Dig. 1, 5, fol. 19: «1. [...] In quaestione prorro status, summa \& prima divisio est, qua homines in Liberos \& Servos tribuuntur, hoc enim primum \& praecipuum iuris status caput es, quod vitae mortique comparatur. Sed quia de discrimine sexuum aliqua hoc in titulo traduntur, nos nocendi gratia divisionem personarum primam a natura mutuabimur. 2. Personarum ergo triplex esto divisio: una Naturalis, altera Iuris gentium, tertia Civilis».

21 Hahn (1668) ad Dig. 1, 5, n. 1, fol. 83: «Vox Status JCtis accipitur duplice. Primo pro firma \& perfecta aetate ... Secundo pro conditione seu qualitate aliqua. Hoc modo sumitur. I. late \& generaliter. Sic dicitur status Reip. [...] status publicus \&privatus. II. Refertur ad hominem, \& accipit iterum tripliciter. I. generaliter denotat hominis conditionem quamcumque, v.c. naturalem, quam quis a natura habet $t$. polit. 10. utpote quae ratione 
Por lo que aquí toca, una de las importantes consecuencias de la consolidación de esta distinción entre status naturalis y status civilis en el usus modernus Pandectarum, fue que ella, a propósito del status naturalis, atraía a la sede del estado de las personas una cuestión que en los textos romanos se discutía especialmente en sede de sucesiones, a saber, la del nacimiento de las personas, y la consiguiente distinción entre ya nacidos (nati) y los que estaban por nacer (nasciturus). Con ello, a su vez, el "tiempo" ganaba un lugar en el campo de statu personarum.

\section{b) En el espacio racionalista de Domat}

El punto de partida de la lectura de Domat se situaba en la siguiente advertencia: aunque las leyes civiles reconocían la igualdad que el derecho natural había constituido entre todos los hombres, sin embargo, se distinguían ciertas cualidades, que se referían peculiarmente a materias del dererecho natural y que constituían los que se llamaba status personarum. ${ }^{22}$ Las distinciones que constituían cualidades que se dirigían al estado de los hombres eran de dos géneros, pues unas eran naturales, y otras habían sido establecidas por los hombres, y de ahí que hubiera un statum secundum naturae y otro secundum legum ordinem. ${ }^{23}$

Dedicaba Domat la primera sección del título De personis a tratar De statu personarum circa naturam, y eran tres las distinciones que admitía este status:

sexus est, maris \& foeminae, l. 9. l. 10. h. nati vel in utero existentis [...] II. Strictius \& specialiter refertur ad statum hominum civilem, seu politicum, qui in societate civili viventibus perspicuam juris diversitatem inducit, s. secundum quam iis diversimode jus redditur. Hic autem a jure est seu lege. Sic status \& dignitas opponuntur [...] III. Strictissime status accipitur pro parte status. Ut pro libertate [...] Ita \& aliquando accipitur pro civitate, nonumquam familia [...]».

22 Dомат (1785) tit. II, pr., fol. 15: «Etsi civiles leges quamdam aequalitatis speciem recognoscant, quae ius naturale constituit inter homines omnes personas tamen distinguunt quibusdam qualitatibus, quae peculiariter ad juris naturae materias referuntur, quaeque id constituunt, quod status personarum appellatur».

23 Domat (1785), fol. 16: «Duplicis generis sunt distinctiones, quas inter homines constituunt qualitates illae, quibus eorum status dirigitur. Aliae enim naturales sunt, ac iis distinctae qualitatibus, quas natura ipsa in quocumque denotat. Natura quippe sexus distinguit, eosque qui hermaphroditi appellantur. Aliae vero distinctionibus humanis fuerunt constitutae. Hinc servitus naturae status non est, eumque homines effinxerunt. Juxta autem varias hujusmodi distinctiones unusquisque proprium habet statum secundum naturae \& legum ordinem». 
Distinctio, personarum ex sexu, ${ }^{24}$ Distinctio ex nativitate \& patria potestate, ${ }^{25} \mathrm{y}$ Distinctio ex aetate. ${ }^{26}$ La sección II la destinaba al examen De statu personarum juxta leges civiles, cuyas distinciones eran establecidas al arbitrio de las leyes, de modo que no tenían ningún fundamento en la naturaleza. ${ }^{27}$ Después de recordar que en el derecho romano eran las consideraciones de la libertas, civitas y familia las que fundaban sus distinciones, advertía que in Galliarum regno la distinción de las personas era diversa, pues lo eran las siguientes: entre nobiles e ignobiles; entre incolae y homines ruris; a las que habían de añadirse aquellas introducidas ex consuetudine in Galliarum regno, que distinguían a los hombres libres de aquellos otros que eran siervos o estaban en condición servil. A propósito de esto no dejaba de interesar la advertencia que hacía para destacar que la distinción de los siervos no tenía ningún fundamento en las cualidades personales, sino sólo en el domicilio de las personas y en la calidad de sus bienes, de modo que las calidades de vasallos y enfiteutas no eran, propiamente, cualidades personales, sino que emanaban o del domicilio o de la naturaleza de los bienes que poseían. ${ }^{28}$

Tal era, en términos generales, el estado de las lecturas que sobre el status se podía observar en la cultura del derecho común durante la segunda mitad del siglo XVII. En ellas, como consecuencia de la mayor libertad con la que los juristas enfrentaron los textos justinianeos, sobre todo de cara a incluir en sus explicaciones la situación de las personas en sus respectivas épocas y sociedades, el tiempo (tempus) y el espacio (spatium) comenzaban a reclamar un lugar entre los criterios que fundaban las distinciones del status de las personas. En efecto, el tempus se abría paso en aquellas distinciones fundadas en el nacimiento (nasciturus/natus) y en la edad (minores/maiores); y, más débilmente, el spatium en aquellas fundadas en la civitas, entendida a la manera de los textos romanos (cives / peregrini), o ex consuetudine de las regiones y lugares (incolae / homines ruris).

24 Domat (1785) tit. II, sect. I, n. 1, fol. 17.

25 Doмat (1785) tit. II, sect. I, n. 2-15, fol. 17-19.

26 Dомат (1785) tit. II, sect. I, n. 16, fol. 19.

27 Dомат (1785) tit. II, sect. II, pr., fol. 19: «Distinctiones status personarum juxta civiles leges, eae sunt, quae arbitrariis legibus constituuntur, seu hujusmodi distinctiones nullum in natura habeant fundamentum, quemadmodum distinctio est personarum in liberos $\&$ servos; seu ex aliqua naturali qualitate nascantur, quemadmodum sunt majoritas \& minoritas».

28 Dомат (1785) tit. II, sect. II, pr. fol. 19-20.

$72 \quad$ Javier Barrientos Grandon 
En esa misma segunda mitad del siglo XVII aparecerá una nueva lectura, que sin estar completamente desligada de las anteriores, se mostrará como singularmente novedosa e influyente. En ella el spatium, desde dos perspectivas diversas, asumía un especial papel en la configuración de la categoría del status de las personas, y de la misma noción de estas. De ello se siguió una importante consecuencia en lo que tocaba a la ordenación del derecho de las personas, cuya influencia puede seguirse, al menos, hasta las primeras codificaciones decimonónicas.

A tal lectura, que fue la de Pufendorf, y a sugerir algunas cuestiones de interés que de ella se derivan por lo que toca al espacio, y en menor medida al tiempo, en relación con el estado de las personas, dedicaré este breve artículo.

\section{Pufendorf y una lectura de la persona y el status desde la "analogía" con las substancias y el spatium}

Samuel Pufendorf (1632-1694), en su De iure naturae et gentium (1672) desde una manifiesta perspectiva filosófica, realizó una lectura de las tradicionales categorías de persona y status y de sus relaciones, en la que el spatium desempeñaba, por una parte, un especialísimo papel "metodológico" y, por otra, jugaba un determinado rol como criterio diferenciador de las personas.

Asumida por Pufendorf la distinción entre los entia physica y los entia moralia, a estos últimos los presentaba como ciertos "modos" (modi quidem), lo que había de entenderse en relación con la substantia, pues los entes morales no subsistían por sí mismos, sino en las substancias. ${ }^{29}$ Tales entia moralia no procedían de los principios intrínsecos de las substancias de las cosas, porque en cuanto tales eran realidades ya existentes que se "sobreañadían”, y de ahí que fuera la palabra 'imposición’ (impositionis) la que mejor

29 Pufendorf (1684) lib. I, cap. I, 3, fol. 3-4: «Exinde comodissime videtur entia moralia posse deffinire, quod sint modi quidem; rebus aut motibus physicis superadditi ab entibus intelligentibus, ad dirigendam potissimum et temperandam libertatem actuum hominis voluntariorum, et ad ordinem aliquem ac decorem vitae humanae conciliandum. Modos dicimus. Nam concinnius nobis videtur ens latissime dividere in substantuam \& modum, quam in substantiam \& accidens. Modus porro uti substantiae contradistinguitur; ita eo ipsa satis pater, entia moralia non per se subsistere, sed in susbtantiis, earumque motibus fundari, ipsasque certa duntaxat ratione efficere». 
podía expresar la producción de los entes morales. Una "imposición" que se daba al arbitrio de los entes inteligentes y gracias a una voluntad superior, para dirigir y temperar la libertad de los actos voluntarios de los hombres y para procurar el orden y decoro de la vida humana. ${ }^{\mathbf{3 0}}$

Esos entia moralia, aunque no subsistían por sí mismos y aunque debían ser tenidos en el universo, no en la clase de las substancias, sino en la de los modos, podían ser concebidos "al modo de las substancias", porque los morales se sujetaban inmediatamente en estas casi por igual razón que la cantidad y cualidades inherían en las substancias corpóreas. ${ }^{31}$ Los entia moralia, que se concebían por analogía de las substancias, se decían "personae morales”, y estas no eran más que hombres singulares, unidos por un vínculo moral en un sistema, de forma que se les consideraba en su status o munus. ${ }^{32}$

De la originaria concepción de las personas, en cuanto que entes morales, ad analogiam de las substancias, se seguía otra concepción analógica, que era la que daba origen a la particular noción de status asumida por Puffendorf. En la analogía esta vez ocupaba un lugar central el spatium. ${ }^{33}$

En efecto, de la misma manera que las substancias físicas presuponían el spatium, en que ponían su existencia natural y ejercían sus movimientos físicos, así, por esta analogía, también las personas principalmente morales, se decían y entendían "esse in Statu". Este status, asimismo, se suponía o

30 Pufendorf (1684) lib. I, cap. I, 4, fol. 4: «Porro uti modus originarius producendi entia physica est creatio; ita modum, quo entia moralia producuntur, vix melius possis exprimere, quam per vocabulum impositionis. Sicilicet quia illa no ex principiis intrinsecis substantiae rerum proveniunt, sed rebus iam existentibus et physice perfectis, eorundem effectibus naturalibus sunt superaddita ex arbitrio entium intelligentium, absque unice per eorundem determinationum existentiam naciscuntur».

31 Pufendorf (1684) lib. I, cap. I, 6, fol. 5: «Etsi autem entia moralia per se subsistant, adeoque in universum non in classe substantiarum, sed modorum sint censenda: deprehendimus tamen quaedam concipi ad modum substantiarum, quia in iis alia moralia videntur inmediate fundari pari fere ratione, qua substantiis corporeis quantitas \& qualitates inhaerent».

32 Pufendorf (1684) lib. I, cap. I, 12, fol. 10: «Entia moralia, quae ad analogiam substantiarum concipiuntur, dicuntur personae morales, quae sunt homines singuli, aut per vinculum morale in unum systema connexi, considerati cum statu suo aut munere, in quo in vita communi versantur».

33 Para la concepción de Pufendorf acerca de la persona moralis y del status, aunque con preocupaciones diversas a las de este estudio, vide Lipp (1982/1983) 218-262, en particular pp. 222-233. 
extendía "bajo ellas", para que en él ejercieran sus acciones y efectos. Así se instituían ciertos estados, no por sí mismos, sino para que en ellos se entendieran existir las personas morales. ${ }^{34}$

El status se mostraba así, ad analogiam, como el spatium en que obraban las personas, y que había de entenderse como una facultad de hacer a la manera de una "cualidad activa", como cuando se concebía a la libertad como status, por analogía con el espacio, si que se cayera en el error de confundirlo con algunos atributos del estado, pues éstos se concebían al modo de cualidades pasivas. ${ }^{35}$ La caracterización del status como una qualitas tenía como consecuencia el que Puffendorf abandonara por completo el recurso a la voz 'conditio', que había sido la usual entre los juristas que habían definido al status, y que aún se conservaba en el usus modernus Pandectarum.

Sobre tales bases, Puffendorf realizaba un lectura del status, en cuanto que cualidad activa, a la luz de su ya explicada analogía con el spatium, y ello le permitía generar una articulada distinción de estados. En efecto, así como el espacio era doble: uno según se dijera que la cosa estaba en un lugar $(u b i)$, como aquí, allí, y otro según se dijera cuándo lo estaba (quando), como hoy, ayer, mañana, debía advertirse que el género del status era, del mismo modo, doble: el uno que denotaba el " $u b i$ morale", y que tenía alguna analogía con el lugar (loco), y el otro que lo denotaba respecto del tiempo (ad tempus), en la medida en que alguno de los efectos morales redundara en aquellos que se decían existir en este tiempo. ${ }^{36}$

34 Pufendorf (1684) fol. 5, lib. I, cap. I, 6: «Verum quemadmodum substantiae physicae velut supponunt spatium, in quo suam quam habent naturalem existentiam ponunt, \&motus suos physicos exercent: ita ad harum analogiam etiam personae potissimum morales dicuntur, \& intelliguntur esse in Statu; qui itidem iis velut supponitur aut substernitur, ut in eo actiones atque effectus suos exserant».

35 Pufendorf (1684) lib. I, cap. I, 11, fol. 9: «Antequam ad alia discedamus, illud quoque momendum videtur, quod ob inopiam vocabulorum uno eodemque nomine requenter cogamur exprimere \& statutm, \& attributum statui proprium. Quae tamen re vera distincta sunt, \& diverso modo concipiuntur [...] Sic v.g. libertas pro statu concipitur ad analogiam spatii; pro facultate agendi ad modum qualitatis activae. Sic nobilitas alias notat statum, alias atributum personae, quod ad modum patibilis qualitatis concipitur».

36 Pufendorf (1684) lib. I, cap. I, 7, fol. 6: «Porro uti spatium duplex est; unum secundum quod res $u b i$ esse dicuntur, aut in loco, v. g. hic, illi, illic; alterum secundum quod dicuntur esse quando, aut in tempore, v. g. hodie, heri, cras: ad eundem modum duplicis esse generis statum animadvertimus, unum qui notat $u b i$ morale, seu qui cum loco aliquam habet analogiam; alterum qui notat respectum ad tempus, quatenus ex eo moralis aliquis effectus redundat in illos, qui in isto tempore existere dicuntur». 
El primer status, análogo al lugar, podía considerarse, o indeterminadamente (indeterminate), en la medida en que sólo resultaba de cualidades morales, o determinadamente (determinate), en la medida en que respecto de la cantidad envolvía alguna comparación moral. ${ }^{37}$

El estado del hombre (status hominis) indeterminadamente considerado era también doble: o natural (naturalis) o adventicio (adventitius). Era el status naturalis entendido, no como el que fluía más allá de toda imposición de los principios físicos de la esencia humana, sino como el que acompañaba al hombre desde el mismo instante de su nacimiento por imposición de la providencia divina y no por arbitrio de los hombres. A este status naturalis se le solía considerar o absolutamente (absolute), en cuanto que opuesto a las bestias, o en orden a otros hombres (in ordine ad alios homines). El status adventitius, de su lado, era el que sobrevenía por algún hecho humano. ${ }^{38}$

En fin, determinadamente (determinate) podían considerarse algunos estados, en cuanto que tuvieran unida una tendencia o remisión de estimación, o en cuanto que se juzgaran por muy o poco honoríficos, pues como a cualquier estado le acompañaban derechos u obligaciones, se estimaba generalmente más espléndido el status que tenía unidos mayores y más fuertes derechos. $^{39}$

37 Pufendorf (1684) lib. I, cap. I, 7, fol. 6: «Prior status loco analogus considerari potest vel indeterminate, quatenus duntaxat ex qualitatibus moralibus resultat: vel determinate, quatenus etiam respectum ad quantitatem aliquam moralem \& comparationem involvit».

38 Pufendorf (1684) fol. 6-7: «Status hominis indeterminate consideratus est vel naturalis, vel adventicius. Naturalem hominis statum vocamus, non quod is citra omnem impositionem ex physicis principiis essentiae humanae fluat; sed quod ex impositione Numinis, non ex arbitrio hominum, hominem statim ab ipsa nativitate comitetur. Solemus autem statum hominis naturalem considerare vel absolute, vel in ordine ad alios homines [...] Huic statu vita \& conditio bestiarum opponitur [...] In ordinem autem ad alios homines consideratus naturalis status ille dicitur, prout intelliguntur homines se invicem habere ex nuda illa \& universali cognatione, quae ex similitudine naturae resultat, ante factum aliquod aut pactum humanum, quo peculiariter unus alteri redditus fuerit obnoxius [...] Adventitius autem status est, qui nascentibus, aut jam natis facto aliquo humano supervenit».

39 Pufendorf (1684) lib. I, cap. I, 9, fol. 9-10: «Determinate considerari possunt aliqui status prout adjunctam habent intensionem aut remissionem existimationis, sed prout multum aut parum honorifici censetur. Cum enim quemlibet statum aliqua jura aut obligationes comitentur: eo splendidior plerumque censetur status, quo plura \& validiora jura is adjuncta habuerit $[\ldots] »$. 
El segundo género de status hominis, considerado respecto al tiempo (ad tempus), admitía dos divisiones, a saber:

a) en junioratum y senioratum, y tanto el uno como el otro se ligaban a la duración de la vida human, que era llamada aetas, y que podía ser: infantia, pueritia, adolescentia, juventus, aetas virilis, stata, vergens, senilis, decrepita; y

b) en majorennitatem, cuando se estimaba que alguno era idóneo para administrar sus propios bienes, y minorennitatem, cuando alguien precisaba de tutor o curador. ${ }^{40}$

Como habrá podido advertirse, el recurso metodológico a la analogía con el spatium había conducido a Pufendorf a ofrecer una lectura en la que, a fuer de su interés para la filosofía, el status personae se mostraba ligado a dos "factores": el lugar (ubi/loco), y el tiempo (quando/tempore).

Las personas podían existir en muchos estados, y en cuanto que concebidas como hombres singulares unidos por un vínculo moral en un sistema, de forma que se les consideraba en su status o munus, admitían una serie de distinciones. Pufendorf, al tratar de tales distinciones, continuaba la preocupación que Vulteius había mostrado por la situación de su tiempo y por hacerlas operativas para explicar la composición de su sociedad.

Me limitaré aquí a enunciar las distinciones que Pufendorf hacía de las personas morales, y expuestas ellas me detendré en los aspectos que interesan a la posición que ocupaban el "espacio" y el "tiempo".

Las personas morales, o eran simples (simplices) o compuestas (compositae). ${ }^{41}$ Estas últimas eran las que se constituían cuando muchos individuos humanos se unían entre sí para que, por la fuerza de esta unión quisieran o actuaran como una sola voluntad y una sola acción, y no se las estimara

40 Pufendorf (1684) lib. I, cap. I, 10, fol. 10: «Posterius genus status, quod respectum ad tempus habet cum effectu morali consideratum, divi potest 1 . in junioratum \& senioratum. Quorum uterque dicitur vel in respectu ad durationem existentiae in vita humana, vocatur aetas; cujus gradus infantia, pueritia, adolescentia, juventus, aetas virilis, stata, vergens, senilis, decrepita: vel in respectu ad statum aliquem adventitium, prout quis diu in eo fuit versatus. 2. In majorennitatem, quando quis per aetatem censetur idoneus ad bona sua proprio Marte administranda; \& minorennitatem, cum quis tutore adhuc aut curatore opus habet, eo quod ob judicii imbecillitatem, aut affectum in vana impetum rebus suis non satis dextre prae esse posse praesumatur».

41 Pufendorf (1684) lib. I, cap. I, 12, fol. 10: «Sunt autem personae morales vel simplices vel compositae». 
como muchos. Como estas personas compuestas podían dividirse del mismo modo que las personas simples, véase lo que se dirá en el párrafo siguiente. ${ }^{42}$

Las personas simples, por razón de su status o munus, podían ser públicas (publicae) o privadas (privatae). En los pueblos cristianos las personas públicas se dividían en politicas y eclesiasticas. Las políticas, a su vez, podían ser principales o minus principales, a las que podía agregarse una "peculiar especie de personas políticas", que era posible llamar repraesentativas, entre las que tradicionalmente había de contarse a los legados, vicarios, síndicos y similares y, modernamente, era preciso distinguir entre aquellos ministros que tenían characterem repraesentantem, como eran los legados, y los ministros de segundo orden, como los enviados o residentes. ${ }^{43}$

En cuanto a las personas privadas, no podía decirse sino que había una gran variedad de ellas, y que sus principales diferencias se derivaban de los siguientes criterios:

$\left.1^{\circ}\right)$ Ex negotio, quaestu aut artificio, es decir, por el negocio, tráfico o arte en que se ocupaban las personas;

$\left.2^{\circ}\right)$ Ex conditione seu situ quasi morali, quo quis utitur in civitate, esto es, por la posición que se tenía en la ciudad, de donde, unas eran cives, de pleno o menos pleno derecho, otras inquilinus, y otras peregrinus;

$\left.3^{\circ}\right)$ Ex conditione in familia, respecto de lo cual algunas eran paterfamilias, otras uxor, otras filius, y otras servus, y que, por así, decirlo, eran los miembros de la familia ordinaria, y a las que alguna vez extraordinariamente se añadía el hospes;

$\left.4^{\circ}\right)$ Ex stirpe, de donde unas eran nobiles, y que eran distintas en las diversas ciudades, al igual que sus grados, y otras plebeji; y

$5^{\circ}$ ) Ex sexu \& aetate, de lo que se seguía que unas eran hombres y otras mujeres, y que fueran: puer, juvenis, vir o senex. ${ }^{\mathbf{4}}$

42 Pufendorf (1684) fol. 12-13, lib. I, cap. I, 13.

43 Pufendorf (1684) fol. 10-11, lib. I, cap. I, 12.

44 Pufendorf (1684) lib. I, cap. I, 12, fol. 1-112: «Privatarum personarum magna esta varietas. Praecipuae illarum differentiae desumuntur 1. Ex negotio, quaestu aut artificio, circa quod quid est occupatus, \& unde rem facit, quod est vel ingenuo dignum, vel cum sordibus conjunctum. 2. Ex conditione seu situ quasi morali, quo quis utitur in civitate. Quo intuitu alius est civis, pleno aut minus pleno jure; alius inquilinus, alius peregrinus. 3. Ex conditione in familia; quo respectu aliquis est paterfamilias, qui mariti, patris ac heri personam simul complecti potest, alia uxor, alius filius, \& servus; quae sunt membra velut 
Así, pues, las personas, en cuanto que privadas, eran consideradas en su status, y aunque este podía ser de muchas especies, había unos criterios principales que podían instituirlos. De ellos, uno se relacionaba con el spatium y otro con el tempus. El primero era el que atendía a la posición y situación (situ) de las personas que operaban en la civitas, y que fundaba la distinción entre ciudadanos, inquilinos y peregrinos; y el segundo era el que miraba a la edad (aetas), pues las personas podían ser niñas, jóvenes, adultas o ancianas.

\section{A la sombra de Pufendorf: Heineccius y su lectura del status}

La lectura que Pufendorf había hecho del status pareciera que no influyó inmediatamente en los juristas germanos de fines del siglo XVII y primeras décadas del XVIII. En general, en las obras de los juristas de aquel tiempo se consolidó la distinción del status, en naturalis y civilis, que era la característica del usus modernus Pandectarum, y que queda referida en el número anterior a propósito de la obra de Struve. ${ }^{45}$ Con todo, ha de advertirse que algún autor se refería a las opiniones de Pufendorf, pero para aclarar que no seguía su entendimiento de ciertas especies de status. ${ }^{\mathbf{4 6}}$

ordinaria familiae, quibus extra ordinem accedit interdum hospes. 4. Ex stirpe; unde sunt nobiles, quorum diversis in civitatibus diversi gradus: \& plebeji. 5. Ex sexu \& aetate. Hinc est puer, juvenis, vir, senex: illinc mas, foemina».

45 Herold (1737) cap. III, sect. I, n. 1, fol. 56: «Circa personas primum occurrit considerandus ipsarum Status qui vel Naturalis est, vel Civilis. Status Naturalis praecipue respicit Sexum \& Nativitatem», y cap. III, sect. I, n. 16, fol. 59: «Status Civilis omnes personae vel sui Juris sunt, qui vel alienae potestati subiectae [...] Qui in aliena potestate sunt, sunt vel in potestate Dominica, vel paterna [...]»; con alguna novedad en GundLING (1723) ad Inst. 1, 5, fol. 58: «III. [...] Multiplex hominum status est; isque naturalis, gentium, civilis familiae. IV. Ratione Status Naturalis homines dividuntur primo in natos \& nascituros [...]», fol. 64: «XXII. Ratione status ex Iure Gentium homines vel sunt liberi, vel servi [...]», fol. 67: "XXXIV. Ratione Status Civilis homines dividuntur in cives \& peregrinos", y ad Inst. 1, 6, fol. 71: «Pertinet haec rubrica adhuc ad statum hominum, \& quidem familiae, cuius intuitu illi sunt vel patrefamilias, vel filiifamilias [...]».

46 Wolzogen (1688) cap. I, n. 35-36, fol. 8: «Intelligimus autem hic significatione status naturalis non illam conditionem, in qua a creatore homo est constitutus, dum eximium prae ceteris animal eundem esse voluit, intellectu sc. \& ratione praeditum, qua ratione ei opponitur vita \& conditio Brutorum vid. Dn. Pufend. De offic. hom. \& civ. l. 2. c. 1. Sed statum naturalem consideramus in contradictione ad statum civilem [...]». 
Fue en Johann Gottlieb Heineccius (1681-1741) en quien la lectura de Pufendorf sobre el status ejercería, como toda su obra, una singular influencia. Heinnecius, en efecto, se ocupó particularmente de aquellas cuestiones en sus Elementa juris naturae et gentium (1737), aunque sus ideas claves ya se hallaban en su explicación de las Instituciones contenidas en sus Elementa Iuris Civilis secundum ordinem Institutionum (1725), y se recibían, igualmente, en sus Recitationes in Elementa Iuris Civilis secundum ordinem Institutionum (1765).

En sus Elementa Heineccius asumía que, en general, el status era una cualidad, por la que cualquier cosa era limitada, y que aquellas cualidades por las que el hombre mismo resultaba limitado, con propiedad, se llamaban status hominis. Aquellas que, por el mismo Dios en cuanto que creador de los hombres, limitaban su alma o su cuerpo, constituían el statum physicum, y aquellas otras que, por la ley, limitaban sus acciones libres, constituían el llamado statum moralem, ${ }^{47}$ que, a su vez, podía ser naturalis o adventitius. ${ }^{48}$ Este status moralis, se correspondía en la lectura de Pufendorf, con aquel primer género de status, que era el que denotaba el ubi morale, es decir, aquel que tenía cierta analogía con el lugar y, en concreto, cuando era considerado indeterminate, esto es, sólo en la medida en que resultaba de cualidades morales. Status, que considerado indeterminate, precisamente Pufendorf había diferenciado entre un status naturalis y otro adventitius.

En sus Elementa Heineccius se había preocupado de advertir en una nota que los jurisconsultos llamaban status naturalis al que él denominaba status physicum, y que denominaban status civilis al que él trataba como status moralis. ${ }^{49}$ Esta era la terminología que él mismo había asumido en sus

47 Heineccius (1746) lib. II, cap. I, 2, fol. 367: «STatus generatim est qualitas, per quam res unaquaeque limitatur: adeoque qualitates, per quas ipse homo limitatur, recte vocantur STATUS HOMINIs. Quemadmodum vero jam vires animi \& corporis ab ipso Deo, hominum conditore, jam actiones eorum liberae per leges limitantur: ita priorem hominum statum PHYSICUM; posteriorem MORALEM adpellamus».

48 Heineccius (1746) lib. II, cap. I, 3, fol. 368: «STAtus porro ille moralis, secundum quem hominem maxime distinguuntur, vel illis connatus est, vel ab aliquo ejus facto pendet. Prior vocatur Naturalis; posterior ADVENTITIUS».

49 Heineccius (1746) lib. II, cap. I, 2, nota *, fol. 368: «Quae quidem omnia ad statum hominis Physicum, quem Jureconsulti naturalem vocant, [...] Quare haec omnia ad statum hominis MORALEM, qui jureconsultis civilis est, [...]». 
Elementa Iuris Civilis secundum ordinem Institutionum y la que se leía en sus Recitationes. Con esta advertencia, Heineccius no hacía más que afirmar que leía aquella distinción de status, característica del usus modernus Pandectarum, desde su propia concepción de status (physicum y moralis), que era la que dependía de aquella lectura de Pufendorf, en cuya base se hallaba la analogía con las substancias materiales y el spatium.

Como en los Elementa iuris naturae et gentium era el status moralis el que le tocaba directamente, ${ }^{50}$ no había en él una explicación propia del status physicus, ni menos era su perspectiva una que estuviera determinada especialmente por el derecho. De ahí que fuera en los Elementa Iuris Civilis secundum ordinem Institutionum y en las Recitationes en las que Heineccius explicaba sistemáticamente ambos estados, y al hacerlo asumía la terminología que habían difundido los juristas del usus modernus Pandectarum, es decir, la de status naturalis y status civilis.

Desde la usual distinción que en el derecho había entre homo y persona, ${ }^{51}$ Heineccius destacaba que homo era cualquier mente dotada de razón y que se correspondía con el cuerpo humano, y que persona era "el hombre cuando era considerado en cierto status" o "considerado en su status", ${ }^{52}$ de lo que se seguía en sus Recitationes la conclusión según la cual quien no tenía status no era persona. ${ }^{53}$

En la senda de Pufendorf, caracterizaba al status como una "cualidad", y con ello se afirmaba en esta tradición el abandono de la descripción del status como una cierta "condición". Cualidad era esta, por cuya razón los hombres usaban de un derecho diverso, porque, por ejemplo, uno era el derecho que

50 Heineccius (1746) lib. II, cap. I, 2, nota *, fol. 368: «[...] statum hominis non physicum, sed moralem, directe ad nos pertinere».

51 Heineccius (1730) n. 75, fol. 43: «Номо \& Persona in jure maxime diferunt»; Heineccius (1773) n. 75, fol. 51: «Homo \& persona grammatice sunt synonima, at iuridice differunt. Omnis quidem persona homo est, sed non omnis homo est persona».

52 Heineccius (1730) n. 75, fol. 43-44: «Номо est, cui cumcumque mens ratione praedita in corpore humano contingit. Persona est homo, cum statu quodam consideratus»; Heineccius (1773) n. 75, fol. 51: «Homo est, quicumque habet mentem ratione praeditam in corpore humano: ast persona est homo cum statu suo consideratus». Insitía en esta caracterización en sus notas al Commentarius de Vinnius, Heineccius (1747), fol. 6, ad Inst. 1, 3: "Ast persona est homo, statu quodam veluti indutus».

53 Heineccius (1747) n. 75, fol. 51: «Qui itaque statum non habet, is nec est persona». 
usaba el hombre libre, otro el esclavo, otro el ciudadano, otro el peregrino, y de ahí se seguía que la libertas y la civitas fueran llamadas status. ${ }^{54}$

El status o era naturalis o civilis. ${ }^{55}$ Status naturalis era el que procedía de la misma naturaleza que, por ejemplo, hacía que unos fueran machos y otros hembras, o que unos ya fueran ya nacidos y que otros estuvieran por nacer. ${ }^{56}$ Status civilis era, en cambio, el que se derivaba del derecho civil, que diferenciaba entre libres y esclavos, ciudadanos y peregrinos, padres de familia e hijos de familia, de manera que este status civilis era triple: libertatis, civitatis, familiae. ${ }^{57}$

Así, en una lectura que sólo se quedara en la mirada del jurista, Heinnecius reafirmaba el papel que el tempus y spatium desempeñaban en el status personae. El tempus se afincaba como uno de los criterios que instituían diferencias en el status naturalis: nacidos y por nacer (nati/nasciturus) y, aunque no lo mencionaba entre sus ejemplos, mayores y menores (maiores/minores). El spatium, por su parte, arraigaba como criterio de distinción en el status civilis: ciudadanos y extranjeros (cives/peregrini).

La simpleza de estas distinciones del status, que recibían manifiestamente la tradición del usus modernus Pandectarum, descansaba en una concepción de las personas que había instaladado Pufendorf y que implicaba una clara ruptura con la tradición anterior. La analogía del status con el spatium, que permitía caracterizarlo como una cualidad le volvía en un cierto "espacio de libertad", pues a no otra cosa conducía entender el status, ad analogiam, como el espacio en el que desenvolvían sus operaciones las personas, que

54 Heineccius (1730) n. 76, fol. 44: «STatus est qualitas, cuius ratione homines diverso iure utuntur [...]»; HeIneccius, (1730) n. 76, fol. 5-152: «Quaeritur itaque: quid sit Status? Resp. Esse qualitatem: cuius ratione homines diverso iure utuntur, e.g. quia alio iure utitur liber homo, alio servus, alio civis, alio peregrinus, hinc libertas $\&$ civitas dicuntur status».

55 Heineccius (1730) n. 76, fol. 44: «[...] isque est vel naturalis, vel civilis»; Heineccius (1730) n. 76, fol. 52: «Status ICtis duplex est, naturalis \& civilis»; Heineccius (1747) ad Inst. 1, 3, fol. 6: «Status vel naturalis est, vel civilis».

56 Heineccius (1730) n. 76, fol. 52: «Naturalis est, qui ab ipsa natura proficiscitur, e. g. quod alii sint masculi, alii feminae, alii nati, alii nascituri vel ventres».

57 Heineccius (1730) n. 76, fol. 52: «Civilis est, qui ex iure civilis descendit, uti differentia inter liberos \& servos, cives \& peregrinos, patres \& filios familias. Hinc status civilis triplex est: libertatis, secundum quam alii sunt liberi, alii sunt servi; civitatis, secundum quam alii cives, alii peregrini; \& denique familiae, secundum quam alii patresfamilias, alii filiifamilias». 
ante todo eran libres, en el giro de Pufendorf, o desde la cara de los términos o límites que señalaban para la actuación libre de las personas, en el giro de Heineccius.

Esa misma simpleza fue uno de los factores decisivos que explica la difusión que la lectura de Heineccius tuvo en diversos espacios europeos y no europeos y, en especial, en el de los reinos de España y de las Indias.

\section{La fortuna de la lectura de Heineccius: el caso de los reinos de España e Indias}

En los reinos de España e Indias desde la segunda década del siglo XVIII hubo una especial preocupación de la corona por que en las universidades se estudiara el derecho real, y así se encargó por auto acordado del Consejo de Castilla de 4 de diciembre de 1713 y se reiteró por auto acordado de 29 de mayo de 1741. Aunque esta medida fue resistida por algunas universidades, una de sus consecuencias fue que, por regla general, se adoptaran los In quatuor libros Institutionum Imperialium commentarius academicus \& forensis de Vinnius para el estudio del derecho romano concordado con el derecho real. $^{58}$

Otra consecuencia de lo anterior fue el que, o se editara la dicha obra de Vinnius "castigada" según las notas del Index hispano, que la había incluido en 1707, o que sirviera de príncipalísima fuente para la redacción de ciertos libros de Instituciones acomodados a la enseñanza del derecho hispano. Entre estos últimos han de contarse las Institutiones Hispanae practico-theorico commentatae (1735) del profesor salmantino Antonio Torres y Velasco, particularmente apegadas al texto de Vinnius; la mucho más alejada Instituta Civil y Real donde se explican los SS de Justiniano y en su seguida los casos prácticos, según las Leyes reales de España (1745) de José Berní Catalá (1712-1787); las algo menos apartadas Elucidationes ad quatuor libros Institutionum Imperatoris Justiniani opportune locupletatae legibus decisionibusque juris Hispani (Méjico, 1787) de Santiago Magro y Zurita (1693-1732) y Eusebio Ventura Beleña (1736-1794); y las nuevamente más cercanas a Vinnius Institutiones Romano Hispanae ad usum tironum Hispanorum ordinatae (Valencia, 1788) de Juan Sala Bañuls (1731-1806). Entre las ediciones hispanas o "castigadas" de Vinnius,

58 Vide, para el caso de las universidades hispanas, Peset (1975); y para el de las indianas, Barrientos Grandon (1993) 38-51, 123-139; Vargas Valencia (2011) 108-113. 
fue la primera la que dio a la imprenta Bernardo Joaquín Dánvila, que era una Editio nova in usum Hispanae iuventutis adornata (Valencia, 1778), y a ella siguió el Vinnius castigatus atque ad usum tironum hispanorum accomodatus (Valencia, 1779-1780) del ya citado Juan Sala Bañuls (1731-1806).

El amplio y dilatado recurso en la enseñanza al Commentarius de Vinnius contribuyó a que durante buena parte del siglo XVIII se difundiera en los reinos de España e Indias su lectura del status, en cuanto que una "condición" o "cualidad" de las personas, que obraba como causa del derecho concreto que le competía, y que su tratamiento y distinciones se mantuviera apegado a la habitual trilogía libertas, civitas, familiae. Así, por ejemplo, en la obra de Torres y Velasco prácticamente se reproducía el pasaje correspondiente de Vinnius, aunque se le añadía algún ejemplo, ${ }^{59}$ y en la de Sala Bañuls la correspondencia con el texto de Vinnius era aún mayor, ${ }^{60}$ y sin que en ninguna de ellas se asumiera la distinción entre status naturalis y status civilis que, como queda dicho, no se hallaba en la lectura de Vinnius.

En la segunda mitad del siglo XVIII, estrechamente ligada a las reformas de los planes de estudios de las facultades de leyes, se abrieron paso oficialmente en los reinos de España e Indias las obras de Heineccius. Sus Elementa iuris naturae et gentium se adoptaron como texto en la mayoría de las facultades en que se estableció la nueva cátedra de Derecho natural y de gentes, y sus Elementa Juris Civilis secundum ordinem Institutionum y, en algunos casos, las Recitationes, o sus "notas" al Commentarius de Vinnius, se adoptaron para la cátedra de Instituta. ${ }^{\mathbf{6 1}}$

$\mathrm{Al}$ igual que el efecto que había generado la adopción para la enseñanza de la obra de Vinnius, la de Heineccius dio lugar a que, en las últimas décadas del siglo XVIII y primeras del XIX, se redactaran nuevas exposiciones

59 Torres y Velasco (1735) ad Inst. 1, 3, fol. 17: «[N]am status ipse est personae conditio, aut qualitas, quae efficit, ut hoc vel illo jure utatur: ut esse liberum, esse servum, esse Ecclesiasticum, esse Saecularem, esse Nobilem, esse plebeium, esse ingenuum, esse libertinum, esse alieni juris, vel esse sui. Itaque status rationem causae jus effecti habet».

60 SAlA (1788) ad Inst. 1, 3, pr., fol. 35: «[N]am status ipse est personae conditio aut qualitas, quae efficit, ut hoc vel illo jure utatur; ut esse liberum, esse servum, esse ingenuum, esse libertinum, esse alieni, esse sui juris. Itaque status rationem causae, jus effecti habet».

61 Vide, para las universidades de los reinos de España, Peset/ Peset (1969) 60-61; Olavide (1989) 132-133; Torremocha Hernández (1993) 147-160, 173-177, 185; Arias dE SaAvedra (1996) 63-69; y para las de los reinos de Indias, Valcárcel (1960) 54-55, 83; Razo Zaragoza (1963) 105-106; Arenal Fenochio (1986). 
del derecho real hispano e hispano-indiano en las que se seguían muy directamente sus Elementa Juris Civilis secundum ordinem Institutionum o sus Recitationes. Tales obras fueron las Instituciones del Derecho Real de Castilla (Madrid, 1771) del aragonés Ignacio Jordán de Asso y del Río (1742-1814) y de Miguel de Manuel Rodríguez (17 ?-1797); la Ilustración del Derecho Real de España (Valencia, 1803) del ya mencionado Juan Sala Bañuls (1731-1806); y las Instituciones de Derecho Real de Castilla e Indias (Guatemala, 1818) de José María Álvarez (1777-1820), que es la más apegada a las Recitationes de Heineccius. ${ }^{62}$

En las citadas tres obras se seguía con particular fidelidad la lectura de Heineccius sobre la persona y su status, y muchas veces pareciera que sobre la base de las Recitationes más que de los Elementa. Si en algo se apartaban o ampliaban la explicación de Heineccius ello se debía a su propósito principal de explicar el derecho real de Castilla o Indias y no el romano, y cuya principal consecuencia fue la de sentar unas distinciones del status que extendían el esquema de Heineccius y en las que destacaba con mayor claridad el papel que en ellas jugaba el tempus y el spatium como criterios de diferenciación.

\section{$1^{\mathrm{O}}$ La consolidación de la noción "heinecciana" de persona}

La caracterización que Heineccius había hecho de la persona, en cuanto que hombre considerado en su status, se asentó definitivamentes en estas obras. Así, Asso y Manuel iniciaban su explicación con una definición de persona que recibía literalmente la lectura de Heineccius: «La Persona es: el hombre considerado en su estado; por lo que se dice que no puede haver persona sin que se considere en uno, u otro estado»; ${ }^{63}$ y Álvarez, prácticamente se limi-

62 Álvarez (1818) "Prologo", s/p [3]: «Por lo que a mi hace, desde que me encargué de la de Instituciones de Justiniano fui formando algunos apuntamientos que me facilitasen la enseñanza, y he aquí como corriendo el tiempo llegué a formar los quatro libros. Seguí el orden de los títulos de la Instituta de los Romanos, no obstante que pudiera adoptar otro mejor, y he procurado acomodarme a las definiciones principios y comentarios de las Recitaciones de Heinecio; por que a mas de encerrar los fundamentos generales de nuestra legislacion, la experiencia de catorce años me ha enseñado, que su método es el mas aproposito para el aprovechamiento de la juventud».

63 Asso y del Río/Manuel Rodríguez (1771) lib. I, tít. I, I. 
taba a traducir el correspondiente pasaje de las Recitationes: «Estas palabras hombre y persona, gramaticalmente son sinónimos; pero juridicamente se diferencian mucho. La palabra hombre, es de mayor extensión que la palabra persona: por que toda persona es hombre: pero no todo hombre es persona. Hombre es todo aquel que tiene alma racional unida al cuerpo humano; $y$ persona es el hombre considerado en algún estado. En este supuesto: el que no tiene estado alguno, no es persona». ${ }^{64}$

\section{$2^{\circ}$ ) La noción de status: entre Heineccius y la fidelidad al derecho real}

En una ley de las Siete Partidas $(4,23,1)$ se podía leer una genérica definición de "estado de los hombres", y a ella fueron fieles Asso y Manuel y Sala, condicionados por su propósito de explicar el derecho real, en cambio Álvarez, aunque remitía a la citada ley de Partidas, asumía la noción de Heineccius. $^{65}$

\section{$3^{\mathrm{O})}$ La asunción de la distinción entre "estado natural" y "estado civil"}

A diferencia del modelo de Vinnius, que había sido el habitual en la tradición hispana e indiana, las tres obras de que aquí se trata asumieron la distinción del status que había transmitido Heineccius, en algunos casos, con una dependencia textual del pasaje pertinente de las Recitationes. Asso y Manuel escribían que: «La variedad de condiciones proviene o de la naturaleza, o de la voluntad de los mismos hombres; y por esto el estado de los hombres es natural, y civil»; ${ }^{66}$ Sala que: «Esta condicion viene, o de la misma naturaleza, o de la voluntad de los hombres, y por eso el estado de los hombres se divide en natural y civil»; ${ }^{67}$ y Álvarez que: «El estado es de dos

64 Álvarez (1818) tit. III, 87.

65 Asso y del Río/Manuel Rodríguez (1771) lib. I, tít. I, I: «Estado es: la condicion, o la manera en que los omes viven, o están, l. 1, tit. 23. Part. 4»; SALA (1803) tit. II, n. 1, 8: «Estado de los hombres no es otra cosa, que: Condicion o manera, en que los omes vives o están, l. 1. tit. 23. P. 4»; Álvarez (1818) tit. III, 88: «Por estado entendemos una calidad o circunstancia por razon de la qual los hombres usan de distinto derecho (Princ. y 1. 1, tit. 23. P. 4): porque de un derecho usa el hombre libre, de otro el siervo, de uno el ciudadano, y de otro el peregrino, de ahí nace que la libertad y la ciudad se llaman estados».

66 Asso y del Río/Manuel Rodríguez (1771) lib. I, tít. I, I.

67 SALA (1803) tit. II, n. $1,8$.

86 Javier Barrientos Grandon 
maneras: o natural o civil. Estado natural es aquel que dimana de la misma naturaleza: v. g. que unos sean nacidos otros por nacer unos varones: y otros mugeres: unos mayores de veinte y cinco años y otros menores. Civil es el que trahe su origen del derecho civil: v. g. la diferencia entre hombres libres y siervos: entre ciudadanos y peregrinos: entre padres e hijos de familia». ${ }^{\mathbf{8}}$

\section{$4^{\mathrm{O}}$ ) El "estado natural" y sus tres distinciones: el papel del "tiempo"}

$\mathrm{Al}$ igual que en el caso anterior, en las tres explicaciones del derecho real se admitía la triple distinción del "estado natural" de las personas, que aparecía esbozada en las Recitationes de Heineccius, si bien en las Instituciones de Álvarez, era simplemente enunciada, ${ }^{69} \mathrm{y}$ desarrollada en las exposiciones de Asso y Manuel y de Sala. Dos de ellas tenían como criterio clave el "tiempo", a saber, la que diferenciaba entre las personas que estaban por nacer y las ya nacidas, y la que distinguía entre mayores y menores.

$1^{\mathrm{a})}$ El tiempo y la distinción entre quien está por nacer y el ya nacido: era esta la primera distinción que tocaba al "estado natural". Así en Asso y Manuel se leía que: «Según el estado natural, los hombres en primer lugar, o están por nacer, o ya actualmente nacidos. De aquellos, por razón de humanidad, está establecido: Que mientras es en favor de ellos lo que se hace, les aproveche como si fuesen ya nacidos, 1. 3. tit. 23. part. $4 » ;^{70}$ y en Sala: «Según el natural estado de los hombres, unos son nacidos, otros por nacer o concebidos en el vientre de sus madres. Estos, quando se trata de su bien o comodidad, se consideran nacidos, 1. 3. d. tit. 23. P. 4». ${ }^{71}$ En ambas obras se explicaban con cierto detenimiento las cuestiones más relevantes acerca de esta distinción, en particular, de cuándo se entendía que se nacía vivo.

68 Állvarez (1818) tit. III, 89.

69 SALA (1803) tit. II, n. 1, 8: «Estado natural es aquel que dimana de la misma naturaleza: v. g. que unos sean nacidos otros por nacer unos varones: y otros mugeres: unos mayores de veinte y cinco años y otros menores».

70 Asso y del Río/Manuel Rodríguez (1771) lib. I, tít. I, I.

71 Sala (1803) tit. II, n. 2, 8. 
$2^{\mathrm{a}}$ El tiempo y la distinción entre mayores y menores de edad:

esta distinción, como consecuencia de una lógica temporal en la exposición, se explicaba como tercera distinción, porque primero se estaba en el estado de nasciturus, luego en el de ya nacido, y en este estado se nacía, o varón o mujer, y luego se pasaba del estado de menor al de mayor.

A propósito de esta distinción que atendía a la edad, Asso y Manuel apuntaban que: «Son los hombres en tercero lugar mayores de 25 años, o menores de edad. Estos se consideran antes, o despues de la pubertad, que en los varones empieza a los catorce años, y en las hembras a los doce [...] Considerados antes de la pubertad, se llaman pupilos [...] y en esta edad se ha de distinguir la infancia, que dura hasta los siete años [...] Desde esta edad hasta los diez años y medio, tanto varones, como hembras, se hallan, y llaman proximos a la infancia; y entonces no se sugetan a las penas [...] Desde este tiempo hasta el de la pubertad se llaman proximos a la pubertad, y ya se consideran capaces de dolo, y malicia, y por consiguiente se sugetan a las penas»; ${ }^{72}$ y, por su parte, en la Ilustración de Sala se decía que: «También se diferencian los hombres según este estado por razon de la edad, en que unos son mayores de 25 años, y otros menores». ${ }^{73}$

\section{$3^{\mathrm{a})}$ El sexo y la distinción entre varones y mujeres}

De ella decían Asso y Manuel que: «Los hombres, en segundo lugar, nacen varones, o hembras; y aunque en caso de duda sus derechos sean iguales, sin embargo, como nuestras Leyes se acomodan a lo que regularmente sucede, estando en mayor grado la prudencia en los hombres, y siendo las mugeres de naturaleza mas fragil, nace de aquí: que sean aquellos de mejor condicion que esta en muchas cosas»; ${ }^{74}$ y Sala: «En segundo lugar hay tambien diferencia, según el estado natural de los hombres entre varones y hembras: las quales tambien se entienden baxo la palabra hombre, a excepcion de aquellos asuntos o negocios en que las leyes las excluyen, l. 6. d. tit. P. 7. Aunque por lo comun y en caso de duda tienen el mismo derecho las hembras que los varones, con todo, por quanto las leyes se acomodan a lo que regularmente sucede, y por lo regular los varones exceden en prudencia y constancia

72 Asso y del Río/Manuel Rodríguez (1771) lib. I, tít. I, III.

73 Sala (1803) tit. II, n. 4, 10.

74 Asso y del Río/Manuel Rodríguez (1771) lib. I, tít. I, III. 
de ánimo a las hembras, y estas tienen la naturaleza mas flaca, hay un axioma que dice: Los varones por razon de la dignidad, y las hembras en quanto aquellas cosas en que excusa la fragilidad del sexo, son de mejor condicion». ${ }^{75}$

\section{$5^{\mathrm{O}}$ ) El "estado civil" y sus distinciones: el papel del "espacio"}

Aunque con diferencias de ordenación, en estas tres exposiciones del derecho real se realizaban un serie de distinciones del estado civil que, de manera general, procuraban incluir a todas las especies de personas que había en los reinos de España y de las Indias y, en particular, el "espacio" se constituía en criterio de diferencia respecto de dos de ellas: "naturales" y "extranjeros", y "vecinos" y "transeúntes".

\section{$1^{\mathrm{a})}$ El "espacio" y la distinción entre "naturales" y "extranjeros"}

Para Asso y Manuel era esta la primera distinción del estado civil de los hombres: «Según el estado civil, se consideran los hombres: I. Como naturales de estos Reynos, y estrangeros», ${ }^{76}$ pues no se ocupaba de la tradicional distinción entre "libres" y "esclavos" por dos razones: «La distinción entre libres, y esclavos, que trahe nuestro Derecho en la part. 4. tit. 21 y 22 se halla desconocida en el dia, a no ser que quiera establecerse por los Negros, que se emplean en Indias en los trabajos de minas, o que se tienen en esclavitud por algún particular; pero aun en esta circunstancia es agena de este tratado». ${ }^{77}$ Sala, en cambio, conservaba como primera distinción la de "libres" y "esclavos”, de la que hacía depender las restantes, pues todas ellas se convertían en estados de los hombres libres, de modo que en su exposición la diferencia entre "naturales" y "extranjeros" era la última: «La quinta subdivision de hombres libres es en naturales de nuestros Reynos, y extrangeros». ${ }^{78}$ Álvarez, como en toda su obra, seguía en este punto la ordenación de Heineccius, y se ocupaba de esta distinción al tratar "Del estado de ciudad", sobre el que decía: «El estado de ciudad es aquel por el qual los hombres son o ciudadanos naturales o peregrinos y extrangeros». ${ }^{79}$

75 Sala (1803) tit. II, n. 3, 9.

76 Asso y del Río/Manuel Rodríguez (1771) lib. I, tít. V, XXII.

77 Asso y del Río/Manuel Rodríguez (1771) lib. I, tít. V, XXII.

78 Sala (1803) tit. II, n. 20, 18.

79 Álvarez (1818) tit. III, $\mathbb{S}$ II, 111. 
En general en las tres explicaciones del derecho real se trataba detenidamente de esta distinción, y de sus efectos. Pero por lo que toca más directamente al propósito de este artículo, importa destacar que, a propósito de ella, el "espacio" ganaba un nuevo lugar en sede de derecho de las personas, y lo hacía a través del "domicilio".

Lo anterior era consecuencia directa de lo dispuesto en una ley recibida en la Nueva Recopilación de Castilla (1, 3, 19), en la que se exigía el domicilio como una condición para adquirir el estado de natural. Así Asso y Manuel, después de advertir que muchas de las cuestiones tocantes a la distinción entre naturales y extranjeros, resultaban ajenas al derecho civil, escribían: «Contentandonos ahora con llamar natural de estos Reynos, según la ley Supletoria, a aquel, que fuere nascido en estos Reynos, e hijo de padres, que ambos a dos, o a lo menos el padre sea asimismo nascido en estos Reynos, o haya contrabido domicilio en ellos, y demás de esto haya vivido en ellos por tiempo de diez años, l. 19, tit. 3, lib I. Recop.». ${ }^{80}$ Lo mismo hacía Sala, que también transcribía la ley recopilada. ${ }^{81}$ Álvarez, por su parte, vinculaba también esta distinción al domicilio, y a él se refería en diversos pasajes: «Aquellos que se miran con los respetos de traer su origen de una misma nacion, se llaman naturales; y fuera de estos, los demas son estrangeros [...] En nuestra España todos los domiciliados se comprehenden baxo la denominacion de españoles, pero sin olvidar que unos son naturales, y otros naturalizados». ${ }^{\mathbf{8 2}}$

No toca al intento de este estudio describir el tratamiento que se hacía del "domicilio" en las explicaciones del derecho real. Sí, en cambio, destacar que su inclusión a propósito de la diferencia entre naturales y extranjeros, además de dar un nuevo papel al "espacio" en el estado de las personas, implicaba una ruptura con la ordenación habitual, pues el domicilio resultaba atraído, desde otras sedes materiae, a esta del estado de las personas.

En efecto, la voz 'domicilium' no había sido particularmente utilizada por los juristas clásicos romanos, y su uso parece que se volvió más frecuente sólo a partir de finales del siglo II d. C. Desde el siglo siguiente, en cambio, se acudía a ella con regularidad en diversos textos,tanto jurisprudenciales como imperiales, normalmente ligados a cuestiones que giraban en torno a municipes, incolae, o coloni, de los que la mayor parte se recibieron en ciertos y

80 Asso y del Río/Manuel Rodríguez (1771) lib. I, tít. V, XXIII.

81 SAla (1803) tit. II, n. 20, 18.

82 Álvarez (1818) tit. III, $\mathbb{S}$ II, 112. 
determinados títulos del Digesto: Ad municipalem et de incolis (50, 1), De muneribus et honoribus $(50,4)$ y De verborum significatione $(50,16)$; y del Codex: De municipibus et originariis (10,39), De incolis et ubi quis domicilium habere videtur et de his qui studiorum causa in alia civitate degunt (10, 40). En estos textos, las genéricas sedes materiae en las que solía situarse el uso de la voz 'domicilium' eran: las que tocaban a determinar a qué cargas (munera) se halllaban sujetas las personas, a los honores de qué lugar podían aspirar, y a qué magistrados se encontraban sujetos. De ahí que, precisamente, el término 'domicilium' compareciera en tales pasajes junto a las de 'origo', 'incola', 'municipes', 'colono' y otras similares.

$2^{\mathrm{a}}$ El "espacio" y la distinción entre "vecinos" y "transeúntes"

Esta distinción, que no hacía más que reconocer una diferencia que se remontaba a los derechos locales del Medievo hispánico y que también se había asumido en las ordenaciones y costumbres locales de las Indias, sólo era tratada por Sala y, a propósito de ella, volvía a destacarse el papel del "espacio" en el estado de las personas y, nuevamente, el "domicilio" reclamaba su lugar en esta sede.

En la Ilustración de Sala se leía que: «La quarta subdivision de hombres libres es en vecinos o moradores, y no vecinos o transeuntes». ${ }^{83} \mathrm{Al}$ explicar quiénes habían de tenerse propiamente como vecinos, aparecía su estrecha vinculación con el domicilio y, por ello, trataba específicamente de su constitución en este punto: «[S]i se toma propia y estrechamente, aquel se dice vecino: Que tiene establecido en algun Lugar su domicilio o habitacion con ánimo de permanecer en él. Este ánimo se presume y reputa probado por el transcurso de 10 años [...] dice Gregor. Lop. que tambien se prueba este ánimo, que constituye domicilio, sin el transcurso de los 10 años, por hechos que lo manifiestan, poniendo por exemplo, si uno vende sus posesiones en el Lugar A, y compra otras en el B, donde transfiere su habitacion. Y mas claramente, si fuere recibido en vecino por el comun de algun Lugar, dando fiadores de que permanecerá en él 10 años, y sujetándose a las cargas y tributos vicinales». ${ }^{84}$

83 Sala (1803) tit. II, n. 19, 17.

84 SAla (1803) tít. II, n. 19, 17-18. 


\section{$3^{\mathrm{a})}$ Las otras distinciones del estado civil}

Sólo para completar el panorama del estado civil que se asumía en estas obras del derecho real, aquí se advertirá que sus otras distinciones eran:

a) "libres" y "esclavos", con la salvedad hecha de Asso y Manuel;

b) "nobles" y "plebeyos";

c) "legos" y "eclesiásticos";

d) las propias del estado de familia, expresadas como: "independientes" y "bajo patria potestad" o "bajo tutela o curatela" en la ordenación de Asso y Manuel, o como "casados" y "solteros", e "hijos legítimos" e "hijos naturales", en el caso de Sala.

En resumen:

a) la adopción de la lectura de Heineccius en estas obras de fines del siglo XVIII y principios del XIX, asentó en la cultura jurídica de los reinos de España e Indias, una cierta concepción del "estado de las personas", que podía ser "natural" o "civil";

b) esta distinción básica era la que permitía incluir en sus diferentes distinciones a las diversas especies de personas que habitaban en los reinos;

c) el "tiempo" se convertía, junto al sexo, en uno de los criterios básicos para definir el "estado natural" de las personas, a través de dos de sus distinciones: ya nacidas y por nacer, y mayores de edad y menores de edad;

d) el "espacio" se afincaba como uno de los criterios que fundaba ciertas distinciones del "estado civil", en las que el "domicilio" desempeñaba un papel central; $y$

e) desde una perspectiva "sistemática", la sedes materiae del estado de las personas se había ampliado a unos campos operativos a los que no había estado ligada en los textos justinianeos, con la consiguiente atracción a ella de una serie de cuestiones que habían tenido su sitio en otras sedes.

\section{A modo de conclusión: Las huellas del espacio y el tiempo en el status}

de las personas en una codificación decimonónicaLa explicación y disciplina del "estado de las personas", en cuanto que reflejaba unas sociedades en las que la diferencia jugaba un papel central, constituyó uno de los puntos sobre los que operó con mayor fuerza el proceso codificador en los primeros decenios del siglo XIX. Las diferencias de status se mostraban como abiertamente enfrentadas con el presupuesto ideológico de la "igualdad", que se 
concretó en el propósito de instaurar un "sujeto único de derecho", pues ya el mismo lenguaje de status personae destacaba la existencia de "divisiones" de personas.

Lo anterior justifica que, a modo de conclusión, me detenga ligeramente en la posición que asumió uno de los codificadores en el ámbito hispano y americano, frente al régimen del estado de las personas que se hallaba consolidado en su cultura, y que no era otro que el que se ha explicado en el número anterior, siempre desde la óptica del papel del "tiempo" y del "espacio" en cuanto al estado de las personas.

En 1855 se promulgó el Código Civil de la república de Chile, cuyo principal autor había sido Andrés Bello (1781-1865), y que en el curso del siglo XIX fue adoptado por la mayoría de las respúblicas americanas. ${ }^{85}$ Bello, que profesaba también la enseñanza del derecho romano, siempre había seguido muy de cerca a Heineccius. ${ }^{86}$ Consta, además, que tuvo a la vista para preparar el Código Civil la Ilustración del Derecho Real de España de Juan Sala, ${ }^{87}$ aunque también acudió en el proceso de formación del libro I "De las personas", junto a otras obras, a las de Savigny. ${ }^{\mathbf{8}}$

Desde muy temprano Bello optó, para la ordenación del Código Civil, por el modelo de las Instituciones. ${ }^{89}$ De ahí se seguía que, tras un título preliminar, se abriera con un "Libro Primero. De las personas". Este libro, a diferencia, del homólogo del Code Civil, no asumía la categoría de "actos de estado civil", sino que se mantenía apegado a la tradición expositiva del “estado de las personas” y, aunque no recurría a ella para rubricar sus títulos, una ligera mirada a sus disposiciones permite comprobar que conservó importantes estructuras y contenidos de la exposición institucional característica de la cultura jurídica hispana e indiana, que se había consolidado a través de la lectura de Heineccius.

En efecto, iniciaba el libro I, un primer apartado, cuya rúbrica era "División de las personas", es decir, se situaba en los modelos y categorías que eran los tradicionales al tratamiento del estado de las personas en la cultura jurídica hispana e indiana. Su artículo inicial, el 54, en su inciso 1o establecía,

85 Vide Guzmán Brito (1982); Guzmán Brito (2000).

86 Vide Hanisch Espíndola (1983).

87 Vide Barrientos Grandon (2009).

88 Barrientos Grandon (2013) 159-161.

89 Vide Guzmán Brito (2005). 
a la vieja manera de Gayo, una summa divisio de las personas: "Las personas son naturales o jurídicas", en este caso por influencia directa de Savigny. ${ }^{90}$ En la primera parte del artículo siguiente declaraba quiénes eran personas naturales: "Son personas todos los individuos de la especie humana, cualquiera sea su edad, sexo, estirpe o condición”, he ahí el tributo a su tiempo. Pero, a pesar de tal declaración, en los artículos siguientes de este título se ocupaba de las "divisiones" de las personas.

Los dos primeras divisiones de las personas naturales, no eran más que aquellas dos que, en principio apoyadas en el criterio del "espacio", habían sido consideradas como propias del "estado civil" en las exposiciones del derecho real hispano e indiano bajo el modelo trasmitido por Heineccius:

\section{$1^{\text {a) }}$ "Chilenos y extranjeros":}

el artículo 55, una vez declarado quiénes eran personas, decía: "Dívidense en chilenos y extranjeros". Repárase aquí en que se trataba de una "división de las personas", y con el más estricto apego al modelo hispano e indiano que se ha explicado en el número anterior, sin que un lenguaje que instituyera diferencias entre las personas, se le presentara como opuesto ideológicamente a la igualdad. En cambió, así se lo había parecido a los codificadores franceses, pues, aunque el Code Civil se ocupaba de franceses y extranjeros en el título I de su libro I, no lo hacía desde la categoría de una "división de las personas”, como tampoco había hecho el proyecto de Código Civil español que había impreso en 1852 García Goyena.

Por la razón anterior, el tratamiento que Bello hacía de esta división no seguía el modelo del Code Civil. Así, tal como habían hecho Asso y Manuel, estimaba que no era propia del derecho civil, y se limitaba a expresar en el artículo 56 que: «Son chilenos los que la Constitución del Estado declara tales. Los demás son extranjeros», y concluía esta distinción en el artículo 57: «La ley no reconoce diferencias entre el chileno y el extranjero en cuanto a la adquisición y goce de los derechos civiles que regla este Código».

\section{$2^{\mathrm{a})}$ "Domiciliadas y transeúntes":}

tal era la "división” que se establecía en su artículo 58: «Las personas se dividen, además, en domiciliadas y transeúntes». Este artículo era el primero 
con el que se inauguraba el apartado 3 de este título I del Código Civil, y en cuya rúbrica se mantenía todo el peso de la tradicional sede del estado civil de las personas, pues era la siguiente: «Del domicilio en cuanto depende de la condición o estado civil de la persona». El domicilio, así, se mantenía ligado al status de las personas, tal como en las obras hispanas e indianas que se han explicado en el número anterior. Nuevamente se apreciaba aquí la diferencia con el Code Civil que, aunque trataba del domicilio (arts. 102 a 111), no lo hacía en cuanto que criterio de división de las personas, y en esta misma línea se situaba el proyecto de Código Civil español impreso en 1852 cuando trataba de la vecindad y el domicilio (arts. 35 a 46).

A aquel artículo 58 seguía una serie de otros que se destinaban a fijar la disciplina del domicilio, sobre lo cual sólo apuntaré que admitía su división en "político" y "civil", pero no en el sentido que lo había intaurado el derecho civil francés, pues el político no era aquel en que se ejercían los derechos de ciudadanía y, por ende, sujeto a las leyes políticas, sino, como se declaraba en su artículo 60: «El domicilio político es relativo al territorio del Estado en general. El que lo tiene o adquiere es o se hace miembro de la sociedad chilena, aunque conserve la calidad de extranjero. La constitución y efectos del domicilio político pertenecen al Derecho Internacional». Esta concepción del domicilio político la había tomado Bello de los Commentaries on American Law del juez estadounidense James Kent (1763-1843). ${ }^{91}$ El proyecto de Código Civil español impreso por García Goyena en 1852, no siguió tampoco al Code Civil en este punto, pues no admitió ninguna especie de división del domicilio, por ajena al cultura jurídica hispana, como el mismo García Goyena se ocupaba en advertir: «En Francia hay domicilio politico y civil; el primero para el ejercicio de los derechos políticos; el segundo para el de los civiles: entre nosotros no se conoce hasta abora tal distincion». ${ }^{\mathbf{2}}$

Inmediatamente a continuación de los artículos que trataban del domicilio, se situaba el título II del libro I del Código Civil chileno, bajo la rúbrica siguiente: «Del principio y fin de la existencia de las personas». Su sola lectura destaca el papel que aquí se asigna al "tiempo". Su primer apartado, bajo la rúbrica «Del principio de la existencia de las personas», se destinaba por completo a tratar de una de las distinciones del tradicional "estado natural": personas ya nacidas, y personas por nacer.

91 Barrientos Grandon (2013) I, 170.

92 García Goyena (1852) 48. 


\section{$3^{\mathrm{a}}$ ) Personas "nacidas" $y$ personas "que están por nacer":}

aunque Bello no declaraba expresamente que esta fuera una "división" de las personas, ella resultaba del inciso 1o de su artículo 74, del que se desprendía quien era una persona nacida: «La existencia legal de toda persona principia al nacer, esto es, al separarse completamente de su madre», y de la parte inicial de su artículo 75, que tocaba al nasciturus: «La ley protege la vida del que está por nacer».

Interesa aquí destacar que, aunque Bello no tratara de esta materia expresamente como una "división" de las personas, sí que sistemáticamente conservaba la posición a la que había resultado atraída en las explicaciones del status de las personas. Ello no había ocurrido así en la tradición francesa, y por esto en el Code Civil se mantenía su tratamiento, aunque sólo de modo indirecto, en sede de sucesiones (art. 725), y en el proyecto de Código Civil español impreso en 1852 por García Goyena se situaba en sede de hijos legítimos (art. 107). Lo mismo podía decirse de las cuestiones a que destinaba Bello el 2 apartado del título II del libro I del Código Civil: «Del fin de la existencia de las personas», pues junto con sentar en el artículo 78 que: «La persona termina en la muerte natural», se ocupaba en el siguiente (art. 79) de los co-murientes, cuya sede dogmática tradicional había sido la de sucesiones, en la que lo había mantenido el Code Civil (arts. 720 a 722) y también el ya citado proyecto de Código Civil español impreso en 1852 por García Goyena (art. 552).

Para acabar, sólo decir que la particular lectura de las fuentes que se ha realizado en este artículo, sugiere levemente el papel que el "tiempo" y el "espacio" han jugado, y aún juegan, en una determinada lectura del estado de las personas, tras la cual, las mismas fuentes, nos han puesto delante una serie de cuestiones, entre otras: el papel de la filosofía detrás de las palabras y categorías que solemos juzgar por "sólo" jurídicas; los ejercicios para explicar la realidad con las palabras y categorías de los textos; el papel de las lecturas en las ordenaciones y sistematizaciones dogmáticas; y la, en fin, pervivencia y vías de tránsito de algunas lecturas y sus insospechadas proyecciones. 


\section{Bibliografía}

Accursius (1559), Institutiones Iuris Civilis, D. Iustiniani Magni Imperio per Triumviros Tribonianum, Dorotheum, ac Theophilum conscriptae: \& Franc. Accursii glossis illustratae, Lugduni apud Antonium Vincentium

Álvarez, José María (1818), Instituciones de Derecho Real de Castilla y de Indias, Guatemala: En la imprenta de D. Ignacio Beteta

Arenal Fenochio, Jaime del (1986), Historia de la enseñanza del Derecho Romano en Michoacán (México) 1799-1910, in: Index 14, Napoli, 263-281

Arias de SaAvedra, Inmaculada (ed.) (1996), Plan de estudios de la Universidad de Granada en 1776, Granada: Servicio de publicaciones de la Universidad de Granada

Asso y del Río, Ignacio Jordán de, Miguel de Manuel Rodríguez (1771), Instituciones del Derecho Real de Castilla [...] Van añadidas al fin de cada Titulo las diferencias que de este Derecho se observan en Aragon por disposicion de sus Fueros, Madrid: En la Imprenta de Francisco Xavier Garcia

Barrientos Grandon, Javier (2009), Juan Sala Bañuls (1731-1806) y el "Código Civil” de Chile (1855), in: Revista de Estudios Histórico - Jurídicos XXXI, Valparaíso, 351-368

Barrientos Grandon, Javier (2013), Código Civil. Concordancias. Historia de la ley. Jurisprudencia. Notas explicativas, I, Santiago de Chile: LegalPublishingThomson Reuters

Barrientos Grandon, Javier (1993), La cultura jurídica en la Nueva España, Méjico: Instituto de Investigaciones Jurídica UNAM

Bicquilley, Pierre (1737), De statu libertatis, civitatis, et familiae, ut et de sponsalibus et nuptiis, Argentorati

Domat, Jean (1785), Leges civiles juxta naturalem earum ordinem; Jus publicum \& legum delectus, I, Venetiis: Sumptibus Francisci ex Nicolao Pezzana

Donnellus, Hugo (1840), Commentariorum de jure civili, I, Florentia: ad Signum Clius

Gambiglioni de Aretio, Angelo (1574), In quatuor Institutionum Iustiniani Libros Commentaria, Venetiis: ad Candentis Salamandrae Insignae

García Goyena, Florencio (1852), Concordancias, motivos y comentarios del Código Civil español, I, Madrid: Imprenta de la Sociedad Tipográfico-Editora a cargo de F. Abienzo

Gundling, Nicolaus Hieronymus (1723), Digesta in quibus rationis principia Jus Romanum et Teutonicum et genuinis fontibus simul ac pragmatica connexa ratione expenduntur confusaque nova et accurata methodo separantur, I, Halae Magdeburgicae: Prostat in Officina Libraria Rengeriana

Guzmán Brito, Alejandro (1982), Andrés Bello codificador. Historia de la fijación y codificación de Derecho civil en Chile, I y II, Santiago de Chile: Ediciones de la Universidad de Chile

Guzmán Brito, Alejandro (2000), La codificación civil en Iberoamérica. Siglos XIX y XX, Santiago de Chile: Editorial Jurídica de Chile 
Hahn, Heinrich (1668), Observata theoretico practica, Ad Matthaei Wesenbecii in L. libros Digestorum Commentarios, I, Helmaestadii: Typis \& sumtibus Henningi Mulleri

Hanisch Espíndola, Hugo (1983), Andrés Bello y su obra en Derecho Romano, Santiago de Chile: Ediciones del Consejo de Rectores de las Universidades chilenas

Harprecht, Johann (1562), In quatuor Institutionum Divi Imp. Justiniani Libros, Commentarii privati, breves ac perspicui, Francofurti, Sumptibus Johannis Beyeri: Typis Aegidii Vogelii

Heineccius, Johann Gottlieb (1747), Ad Arnoldi Vinnii Commentarium in quatuor libros Institutionum Imperialium. Additiones \& Notae, s/1

Heineccius, Johann Gottlieb (1730), Elementa Juris Civilis secundum ordinem Institutionum, Giessae: Apud Io. Philipp. Kriegerum

Heineccius, Johann Gottlieb (1746), Elementa Juris Naturae, et Gentium, commoda Auditoribus Methodo adornata, Venetiis: Ex Typographia Balleoniana

Heineccius, Johann Gottlieb (1773), Recitationes in Elementa Iuris Civilis secundum ordinem Institutionum, Vratislaviae: Impensis Io. Friederici Kornii

Herold, Johann Christoph (1737), Tractatus novus de jure ratificationis sive ratihabitionis, Lipsiae: Sumptibus Haeredum Friderici Lanckisii

Hotman, FrançoIs (1569), In quatuor libros Institutionum Iuris Civilis, Basileae: ex officina Hervagiana

Lipp, Martin (1982/83), 'Persona moralis', 'Juristische Person' und 'Personenrecht' - Eine Studie zur Dogmengeschichte der 'juristischen Person' im Naturrecht und frühen 19. Jahrhundert, in: Quaderni fiorentini per la storia del pensiero giuridico moderno $11 / 12,217-262$

Mynsiger a Frundeck, Joachim (1595), Apotelesma, corpus perfectum scholiorum ad Institutiones Iustinianeas pertinentium, Helmaestadii: ex Officina Iacobi Lucii

Olavide, Pablo (1989), Plan de Estudios de la Universidad de Sevilla (Edición de F. Aguilar Piñal), Sevilla: Universitad de Sevilla

Pérez, Antonio (1639), Institutiones Imperiales erotematibus distinctae et explicatae. Rationibus ex principiis Iuris passim depromptis, Lovanii: Apud Everardum de Witte \& Ioannem Vryenborch

Peset, Mariano, José Luis Peset (1969), El reformismo de Carlos III y la Universidad de Salamanca. Plan general de estudios dirigido a la Universidad de Salamanca, por el Real y Supremo Consejo de Castilla en 1771, Salamanca: Universidad de Salamanca

Peset, Mariano (1975), Derecho romano y derecho real en las universidades del siglo XVIII, in: Anuario de Historia del Derecho Español XLV, Madrid, 273-339

Pichardo de Vinuesa, Antonio (1630), In quatuor Institutionum Imperatoris Iustiniani libros, Valladolid: Ex officina viduae Francisci Fernandez de Cordova

Pufendorf, Samuel (1684), De Jure naturae et gentium. Libri octo, Francofurti ad Moenum: Sumptibus Friderici Knochii 
Razo Zaragoza, José Luis (1963), Crónica de la Real y Literaria Universidad de Guadalajara y sus primitivas constituciones, Guadalajara: Universidad de Guadalajara, Instituto Jalisciense de Antropología e Historia

SAla, Juan (1803), Ilustracion del Derecho Real de España, I, Valencia: en la Imprenta de Joseph de Orga

Sala, Juan (1788), Institutiones Romano-Hispanae ad usum tironum hispanorum ordinatae, Valentiae: Typis Salvatoris Faulí

Saxoferrato, Bartolus (1538), Commentaria in primam ff. Veteris partem, Lugduni: Vincentium de Portonariis

Struve, Georg Adam (1692), Syntagma Jurisprudentiae secundum ordinem Pandectarum concinnatum [...] Editio post varias hactenus adornatas concinnior, Jenae: Sumtibus [sic] Matthaei Birckneri, Bibliopolae

Torremocha Hernández, Margarita (1993), La enseñanza entre el inmovilismo y las reformas ilustradas, Valladolid: Universidad de Valladolid

Torres y Velasco, Antonio (1735), Institutiones Hispanae Practico-Theorico commentatae, Matriti: Apud Haeredes Joannis Garcia Infanzon

Ubaldi, Baldo Degli (1586), Commentaria ad quatuor Institutionum libros, Augustae Taurinorum: Apud haeredes Nicolai Bevilaquae

Valcárcel, Daniel (1960), Reformas virreinales en San Marcos, Lima: Universidad Nacional Mayor de San Marcos

Vargas Valencia, Aurelia (2011), Las Instituciones de Justiniano en Nueva España, Méjico: Universidad Nacional Autónoma de Méjico

Vinnius, Arnoldus (1665), In quatuor libros Institutionum Imperialium Commentarius Academicus \& Forensis, Amstelodami: Apud Danielem Elzevirium

Vulteius, Hermann (1613), In Institutiones Juris Civilis a Justiniano compositas Commentarius, Marpurgi: Apud Paulum Egenolphum

Wesenbeck, Mattaeus (1585a), In Pandectas Iuris Civilis, \& Codicis Iustinianei, Lib. iix. Commentarii, Lugduni: In offic. Q. Philip. Tinghi: apud Simphorianum Beraud et Sthephanum Michaëlem

Wesenbeck, Mattaeus (1585b), Institutionum D. Iustiniani, Sacratiss. Principis P. P. A. Libri IIII, Basileae: Per Eusebium Episcopium \&Nicolai fr. Haeredes

Wolzogen, Johann Christoph von (1688), Dissertatio academica de Quaestione status, Francofurti ad Viadrum: typis Christophori Zeitleri

Zasius, Uldaricus (1539), In Primam Digestorum Partem paratitla, Basileae: apud Mich. Ising 

Alejandro Agüero

\title{
Local Law and Localization of Law
}

\author{
Hispanic Legal Tradition and Colonial Culture \\ $\left(16^{\text {th }}-18^{\text {th }}\right.$ Centuries)*
}

\section{Introduction}

The first issue to be considered in a study related to the historical concept of local law is the different cultural conditions of the pre-contemporary normative language. We have to be aware of the particular conditions of a cultural background in which the normative value of legal norms was not exclusively related to some formal feature, like the institutional authority which enacted them, but rather to a hazy consensus on their intrinsic justice, according to the context of production or application. ${ }^{1}$ As it has been noted, many difficulties associated with the study of the colonial local law are related to the perspective from which legal historians of the nineteenth and twentieth centuries have looked at the past, focusing their interest, with rare exceptions, in those phenomena comparable to the contemporary state law. ${ }^{2}$ From a traditional point of view, characterized by a double normativist and state-centered approach, the dimension of local law in the pre-contemporary experience has been usually overlooked or described with conceptual tools not entirely suitable for its thorough comprehension.

The criticism towards the state-centered paradigm that arose during the last decades of the 20th century has promoted a significant change on the coordinates for the historical study of the forms of power and institutional

* A first version of this paper was prepared for a project about the problem of local law in the Spanish colonies of Río de la Plata and published in Tau Anzoátegui / Agüero (2013).

1 On the medieval roots of this topic, Vallejo (2009) 1-13.

2 Tau Anzoátegui (2005) 231-253. We use the terms colonial law to make reference to the Spanish legal culture in the colonial domains. This approach does not include the point of view of the conquered natives but that of the settlers and its relationship with Metropolitan authorities. 
languages. ${ }^{3}$ Accordingly, once the gravitational effect of the state - as naturalized unit of measure on the institutional field - has been removed, two different but substantially related areas have recovered prominence: the great empires and local sphere. As in social studies of the current times, where the increasing interest for the global order has been accompanied by the emergence of communitarian positions (so at the theoretical level as in political struggles for regional identities) it can be said that historiography is also looking with new eyes at the great imperial horizons and the varied world of the local spaces that bubbled beneath them.

The current interaction between supranational, regional and local legal systems, has led to recover the analytical value of some essential aspects of the pre-contemporary experience, such as the jurisdictional complexity and the normative pluralism, that is to say, the coexistence of different normative orders with incidence on the same subjective, material and territorial dominions. ${ }^{4}$ Considering the current «legal pluralism», some scholars have used the metaphoric image of a «new middle ages» to warn about the risk involved by «localisms and universalisms» over the standards of the European liberal criminal law. ${ }^{5}$ And in fact, the coexistence of one universal law along with different local laws, was one of the defining features of the long lasting legal culture of Ius Commune which, rooted on its medieval settings, was kept in force to a great extent during the modern centuries.

Focusing our analysis on the Hispanic legal tradition, particularly, on the colonial experience, we seek in this paper a dual purpose: first, to outline a concept of local law according to the theoretical framework of temporal context, and, second, to point out the cultural factors that converged to bring on a general effect of localization of the law over whatever kind of normative standard. We will try to figure out the relationship between those two different but complementary aspects: on the one hand, the concept of local law, addressed to define, from a synchronic point of view, a certain type of norms that can be qualified prima facie as local; on the other, the notion of localization, useful to describe the dynamic process of interpretation of the general order performed by local authorities in their daily work. After outlining the conceptual framework, we will analyze some examples taken from

3 Hespanha (1986) 191-227; Garriga (2004) 13-44.

4 Benton (2002); Sassen (2006).

5 Donini (2003) 92-119. 
the colonial experience of a frontier jurisdiction such as Córdoba del Tucumán located in the hinterland of the current Argentinean territory.

\section{Local Law: Statutes, customary law and privileges}

In defining a concept of local law that should fit with the Spanish colonial experience, we need to take a look at the cultural horizon of Ius Commune and its imprints in the legal tradition of Castilian conquerors. ${ }^{6}$ As is well known, despite its dual universal slope derived from its Catholic foundations and its Roman imperial legacy, the doctrine of the Ius Commune had to be adapted to the discontinuous nature of the socio political context in which it was spread in medieval Europe. In this horizon, self-regulation of each human community (understood in organic terms) was a basic intuition fully integrated into the theories of the medieval jurists. As it has been rightly pointed out, the medieval culture «could not imagine a universitas, populus, community» that did not have «its own rule, and that was not a whole with it». ${ }^{7}$ With these words, Ugo Nicolini stressed the indissoluble connection between communal self-government and ius proprium (own law) in the context of the medieval northern Italian cities, during the rise of the Ius Commune culture.

In that context, the Roman categories related to territorial organization had to conform to the plural and polycentric nature of the medieval order. ${ }^{\mathbf{8}}$ The Roman concept of province was assimilated by the Commentators both to the district of the Archbishop, in ecclesiastic matters, and to the kingdom in the secular jurisdiction; but even more, in the light of the provisions set by the Peace of Constance (1183), the term province was also equivalent to that of civitas, with the consequent recognition of customary and statutory law of the signatory cities from the Lombard league. ${ }^{9}$ In this case, the local

6 For a recent discussion on the issue of whether the European Ius Commune tradition is valid to analyze the colonial experience, see Hespanha (2006) and (2007), and the opposite perspective expressed by Clavero (2012). In the line of Hespanha thinking, see for the Portuguese-Brazilian case Garriga/Slemian (2013).

7 Nicolini (1982) 249.

8 On the polycentric nature of the medieval space, Kantorowicz (1985) 189-223. On the concept of politic space in the Iberian legal tradition, Hespanha (1993) 85-121.

9 Vallejo (1992) 135-136. 
law of each city (statutory or customary law) was comparable with the ius proprium of a whole kingdom. At the same time, this last expression would be reserved to designate the territorial law developed by each of the kingdoms excluded from the Holy Roman Empire, stressing the contrast between their ius proprium and the Ius Commune. Nonetheless, in the extent in which the kings «in their kingdoms» were equalized to the Emperor «in his Empire», ${ }^{\mathbf{1 0}}$ the theoretical relations between Ius Commune and ius proprium provided a framework to think about, and to deal with, the sometimes conflictive relations between the royal territorial law and a diverse set of localized legal expressions such as fueros, local statutes and customary law.

The normative standards of the Ius Commune addressed to deal with the relationship between princeps and populus, such as the lex ommnes populi (Digest 1, 1,9) and the lex regia de imperio (Digest 1, 4, 1), including also the precepts of the Peace of Constance, were still useful among the Castilian jurists in the early centuries of the Modern Ages. In his renowned glosses of 1555 to Las Siete Partidas, Gregorio López invoked those standards when commenting a key passage of that medieval legal text: the law 12, title I, Partida I, in which it was steadfastly stated:

«An Emperor or King may make laws for the peoples of his dominions but no one else has the power to make them with regard to temporal matters, except where they do so with their [Emperor or kings] permission ...». ${ }^{\mathbf{1 1}}$

In the same way that medieval jurists did when commenting the Roman text (Codex 1, 14, 12, 3: «leges condere soli imperatori concessum est») that inspired Alfonse the Wise's law, Gregorio López warned that such law was only with regard to "general laws for the whole kingdom» and, consequently, that it should not be interpreted as forbidding the villages, cities and other places to make their own statutes. The gloss by López added that the commented law was not related to this kind of local norms which were, instead, regulated by the Ius Commune according to which it was allowed to cities, villages and places to make their own statutes («secundum quod per multas leges permittitur populis condere statuta»). ${ }^{\mathbf{1 2}}$

10 This principle is expressed in the Castilian medieval law, in Las Siete Partidas, Part II, title I, laws 5 and 8 .

11 I have taken the English translation of Las Siete Partidas from Burns (2001) 5.

12 López (1555) (1985), gloss «sobre las gentes de su señorío» in Part I, title I, law 12. 
A nineteenth-century edition of Las Siete Partidas, with glosses translated into Castilian, provides a hint on the enduring significance of that expression related to local statuta. Where Gregorio López, in 1555, said: «Si tamen sint statuta particularia locorum, villarum \& civitatum regni, non tollit illa hæ lex», the 1843 Castilian translation said: «Si son, empero, estatutos [leyes ó fueros, ú ordenanzas] particulares de lugares, villas y ciudades del reino no los impide esta ley». ${ }^{\mathbf{1 3}}$

So, in the long horizon of the Hispanic legal culture, the reflections of medieval jurists on the statutory power of the cities could still provide a base to describe the nineteenth century legal particularism as it is shown by the words between brackets introduced in the translation to denote the meaning of the Latin word statuta. In the background, it was still present the persistent medieval reading of the Codex $(1,14,12,3)$ which allowed to conciliate the opposite extremes: on the one hand, the exclusive legislative power of the Princeps and, on the other, the natural power of self-regulation assigned to every human community. While a Prince or King could make general laws for the kingdom by Himself - not needing other concurrent will (that was the sense attributed to «soli imperatori») - local statute had to be the outcome of a common consensus of the city's councils. ${ }^{14}$

Beyond the many issues that were the subject of discussion on this topic, the aforementioned reasoning also serves to point out the conditions that ruled the enunciation of a power to make general laws and the limitative sense in which was understood the adjective general: because of their being general such laws did not affect, at least in a direct or immediate way, local statutes. In addition to this, in the enduring lexicon of jurists, the word lex was commonly used to make reference to both general and local norms. In that context, from the beginning of the Ius Commune times, it was theorized the very notion of a particular, especial or - properly - local law (legem locale) to define the self-government and local power ascribed to every populus identified as a universitas. In this legal tradition, when jurists had to

13 «However, particular statutes [laws, fueros, or ordinances] from places, villages and cities of the kingdom are not forbidden by this law», SANPONTS y Barba (1843-1844) Gloss 58, Part I, title I, law 12.

14 Nicolini (1982) 262. On the difference between individual legislative power of the Prince and the common or collective nature of the local statutory power, Vallejo (1992) 205-233. 
face the question about who was empowered to make laws, the distinction between general and local law («lege speciali sive locali aut lege propia») was immediately brought into play. ${ }^{15}$

Within this cultural framework, some key elements related to the principles that ruled the relationship between general and local law should be highlighted:

A) The said relationship was not primarily thought in terms of normative hierarchy (although this argument eventually came to be used to repeal some local customs), but rather as an integrative criterion according to which the local law, as specific law, had precedence over the general laws that, in turn, were intended to play a subsidiary function of integration. It is precisely this approach that explains the acceptance of a contra legem consuetudine, understood as an expression of local law that had precedence because of its character of special law (lex speciali). ${ }^{\mathbf{1 6}}$

B) At the core of the original notion of local law, the consent of the populus (always embodied in their natural representatives) plays a key role regardless the fact that to make local statues in the cities without jurisdictional privileges it was formally needed the further approval of the land lord, delegate of the prince or a royal confirmation. ${ }^{\mathbf{1 7}}$

C) As the faculty of making laws was conceived as part of the exercise of jurisdictional power («making laws is an act of jurisdiction» repeated the doctrinal sources), local law was conditioned by the range and degree of jurisdiction that every populus was endowed with, according to its own privileges, customs or other specific title.

Following the last principle, normative powers of cities could be subjected to different limitations. It was considered, for example, that penal laws imposing afflictive or death penalties were reserved to the supreme royal jurisdiction, exceeding the powers of a local community. Apart from that, in some cases, as cities without jurisdictional privileges, it could be distinguished the local statutes that required the intervention of a royal delegate (or royal confirmation) from those norms passed for the oecono-

15 Vallejo (1992) 215.

16 Nicolini (1982) 259.

17 See the reference to the consent of the populus in López (1555) (1985), gloss «sobre las gentes de su señorío", Part I, title I, law 12. 
mic - domestic - government which were part of the exclusive competence of the local council and did not require such intervention or confirmation. ${ }^{\mathbf{1 8}}$

These general principles could be subject to multiple conditions, according to cases and circumstances, particularly in the Castilian Monarchy in which the royal power was consolidated at the beginning of the Late Middle Ages. ${ }^{19}$ However, it would be wrong to think that the early consolidation of royal power involved the dissolution of local regulatory powers. On the contrary, it should be noted that the intervention of the King's power in the production of local law was conceived as a collaborative role with local authorities. Thus, the act of establishing local ordinances reflected, in most cases, the exercise of a power shared by the cities and the Crown. ${ }^{20}$

It is also important to remark that during the second half of the eighteenth century, despite the increasing royal authority and the trend towards legal unification, the distinction between general law and local or particular law was still active as a fundamental criterion of enunciation of the normative order. Let's see, for example, a work issued at the edge of the Ancient Régime, in which is possible to find elements of a new methodology (mixed with more traditional assessments) like the famous «Instituciones del Derecho público general de España con noticias del particular de Cataluña» by Ramón Dou y de Bassols. ${ }^{21}$ While the distinction general/particular was present since the very title of the book, the author made reference to it in two passages denoting its persistent significance. In the first reference, Dou stated that local law was not the subject of his work:

«What is part of the local law of a city, or a province, without extending at least to all provinces of Castile or to the whole principality of Catalonia, is not subject of this work nor should be found things belonging to the Indies except those which are intimately connected with our continent ...». ${ }^{22}$

18 AgüERo (2013) 160-163.

19 Mackay (1985).

20 This collaborative function was graphically expressed by Castillo de Bobadilla who argued that the authority to determine the necessity of reforming the local statutes belonged «copulatively to Corregidor [royal delegate] and aldermen, and not to one without the others", Castillo de Bovadilla $(1597,1704)$ (1978), book III, chap. VIII, $\$ 155$, v. 2, p. 154. On this topic, Agüero (2013).

21 Clavero (1978) 307-334; Tau Anzoátegui (1992a) 473.

22 Dou y de Bassols (1800) I, LIV. 
Despite the secondary importance that they seem to assign to local law, the words by Dou quoted show that the concept still kept its polysemic value, being referable to a city, a province or to all the provinces of a kingdom within the Monarchy. Perhaps only the latter would be considered as public law - the main subject of Dou's concerns. However, when analyzing the specific topic about «the Laws», he claimed:

«The most interesting distinction about the object we are considering is the fact of being some laws general and other particulars».

Dou invoked the principle according to which the laws should fit the circumstances of place, times, customs and the "genius of the subjects», arguing that the Romans, because of their prudence and wisdom, had allowed municipalities to be governed by their own customs, adding that the same was usual in his time in states of vast extension like Spain. ${ }^{23}$ This is just an example to show how in the latter expressions of the ancient regimen's Spanish legal doctrine, the concept of law did not work yet as an exclusionary concept with regard of norms of different range and hierarchy that were a byproduct of an irreducible diversity of localized normative powers.

Besides the underlying particularism, which we will refer later, it must be remarked on now that that double face of law - general and local - was, in turn, the spitting image of a social structure in which, what could be considered the public sphere (using this concept in its widest sense) was still composed, discontinuous, aggregative, and grounded on a basic duality: the powers of the king and the powers of local corporations or republics. ${ }^{24}$ This duality is evident in the medieval legal texts where several prerogatives, nowadays usually attributed to the public administration, were assigned to the king and the cities in such a way that both were casuistically treated as different but equivalent public powers. ${ }^{25}$ The long standing persistence of

23 Dou y de Bassols (1800) I, 64-66.

24 On the public sphere in colonial Latin America, see Schaub (1998) 27-53. On the relationship between Monarchy and republic, see Lempérière (2004) and ZARKA (2007).

25 Consider these examples taken from Las Siete Partidas: in Part III, title XXIII, law 10, is recognized the procedural privilege of suspending the time limit for appeal to those who were out due to a king's commission or «en pro comunal de su concejo», that is to say, a city. In Part III, title XXIX, law 7, is stated that royal revenues are not susceptible to be acquired by prescription and the same privilege is given for squares, roads, pastures, suburbs, and other similar places that belong to the «common of people» (that means, to a 
this dual structure was anchored in the condition of body politics (republics) assigned by natural law to the cities which, upon that condition, kept a dialogic relationship with the king. In this sense, still in 1800, Dou said that the perfect Monarchy's constitution was a combination of aristocracy and democracies. $^{26}$

Grounded upon the feudal logic of exchanging services for privileges, the range of local power could vary from one place to another according to the historical negotiations between the Crown and every city, town or village. ${ }^{27}$ From this perspective, there is another level of local law in the huge amount of privileges conceded by the King that were considered as an acquisition and integrated into the normative set of self regulation of every place. The settlement of a city in the colonies was nothing but the establishment of a republic by means of privileges conceded in the same terms that those used during the times of the medieval fueros. The new republics were empowered with ordinary jurisdiction and mero mixto imperio alongside with a set of franchises and freedoms that constituted the grounds of a first local power that could be developed afterwards through customs and local statutes. ${ }^{28}$ Even more, for those acts exceeding the local competences, the original local power could be increased by negotiating new privileges with the Crown or by obtaining special provisions from the district Royal Courts (Reales Audiencias). ${ }^{29}$

If at a first glance the difference between royal privileges and local ordinances and customs seems to be clear, the line was not so neat. We have already made reference to the collaborative intervention of the royal jurisdiction to authorize or confirm local statutes. This was a contact point

city council). In Part IV, title XV, law 5 is conceded the privilege of legitimating the natural son who was given by his father for the service of «the Emperor's Court, the King, a city council or some other dignity». In Part V, title XIV, law 26, the «King's Chamber» and a «city council» are equalized in order to state that tax debts (caused by "pecho o derrama») cannot be compensated. In Part VI, title IX, law 13, is established that sacred things belonging to the Church cannot be object of a legacy, and same is valid for goods of the King or belongings of «cities or villages».

Dou y De Bassols (1800) I, 18.

27 Fortea Pérez (1991) 119.

28 AGÜERO (2008) 49-58.

29 On the concept of privilege and its significance in the institutional organization of the colonial world, see Rojas (2007). 
between royal privileges and local statues. But the distinction could also be dissolved through certain mechanisms involving the fictitious attribution to the Prince of institutional acts that were an exclusive product of the local practice. Notions like the tolerance or the tacit consent of the Prince made it possible to attribute to the King norms that were the outcome of local practice, equalizing, this way, local customs with royal privileges. Theoretically the convergence was given by the condition of lex speciali assigned to both the royal privileges and the local customs. According with the Ius Commune opinions, Castillo de Bobadilla in 1597 said: «Privilege and customs are equivalent in law, and custom still has the strength of special privilege». ${ }^{30}$ Thus, privileges, customs and local practices - consented or tolerated by the Prince -, were pieces of the complex set of local law whose elements were barely distinguishable.

Finally, it should be noted that if customs and privileges are expressions of local law mostly related to small districts like cities, in some cases they could have a regional dimension being also predicable from a province or a whole kingdom. In the Castilian experience this was particularly evident with the late medieval fueros extensos or general privileges. In this cases, the difference between local or provincial law, and even with the own law of the kingdom (ius proprium) becomes obscure. In the colonial world is usual to see references to the customs of a province or region (i. e. «las costumbres del Perú») and, even more, the whole law passed to rule the colonial domains were sometimes qualified as a «municipal law» or «particular law» within the Castilian law. ${ }^{31}$

\section{The dynamic dimension of local law: localization of law}

The concepts we have been using up to this point (privileges, customary law, statutes, etc.) were shaped by centuries of doctrinal disquisitions. However, they come used to describe issues related to local law from a static perspec-

30 Castillo de Bovadilla $(1597,1704)$ (1978), book III, chap. VIII, $\$ 194-196$. The equivalence between customary law and privilege was also expressed in an ancient law included in the 1567 New Compilation of Laws of Castile (Nueva Recopilación). See Recopilación de las leyes destos reynos (1567) (1640), book VII, title II, law 5. About tolerance and tacit consent of the Prince, some examples can be seen in AgüEro (2013) 150-152.

31 Bravo Lira (1988) 9-10; Barrientos Grandon (2000). 
tive. For this reason, they are not enough to point out the complex interaction of factors that framed the local determination of law. In order to do this, we must look at the cultural conditions that made it possible to put on act the legal field in dynamic terms. ${ }^{32}$ Just from this point of view we are going to be able to consider the full potential that particularism could reach in the general legal framework of the ancient régime.

As we know, for its institutional structure and its cultural conditions, in the pre-contemporary legal culture rules did not work as rigid abstract standards even when they were part of the so called general law of the kingdom. It is also known that in the cultural context we are dealing with, the defining element of making the law was not mainly referred to a kind of norm but to the function of justice. ${ }^{33}$ A substantive conception of justice imposed, in turn, the need of a constant process of interpretation aimed to adjust the rules to the precise conditions of time and place. In order to describe the particularist effect derived from such conception of law and justice, we will use the notion of localization, which means, according to the Oxford dictionary (second meaning) «make (something) local in character». ${ }^{\mathbf{3 4}}$

The notion of localization is used today in different areas of knowledge. In the software industry it is used to denote the process of adaptation of a product to satisfy the cultural, idiomatic and other requirements of a specific local market. ${ }^{35}$ In the field of social sciences it has been used by scholars concerned with globalization phenomena; the concept of localization comes often used as a correlative term of globalization, both in sociology and anthropology. ${ }^{36}$ In the context of historical disciplines, Oliver W. Wolters used it to analyze the process of diffusion and appropriation of ideas amongst different cultures in his studies on cultural history of Southeast Asia. ${ }^{37}$ In the same field, it has been most recently suggested that the concept of localization has a wider analytic potential in comparison with other alternatives like grafting, framing or transplantation of norms, since it stresses the dynamic process of norms diffusion and emphasizes the agency role of

32 For the meaning legal dynamics, in spite of the different context, see Kelsen (1982) 203-204.

33 LACCHÈ / MecCarelli (2012) 9.

34 http:/oxforddictionaries.com/definition/localize; Agüero (2012) 201.

35 See Localisation Industry Standards Association (LISA), quoted by Arevalillo Doval (2000).

36 About localism and globalization see Sousa Santos (1998).

37 Wolters (1982). 
local actors in performing those processes according to their cognitive conditions, beliefs and interests. ${ }^{38}$

In general terms, the notion of localization makes perfect sense in the context of a transnational normative order, with hegemonic features, imposed in peripheral areas by virtue of multiple forms of adaptation resulting from the active involvement of local agencies. ${ }^{39}$ Undoubtedly, this is not exactly the context we have in mind when we talk about the Spanish ancient regime and its colonial culture. Nonetheless, as we have said before, there are some parallelisms between the current global order and the ancient legal culture that may authorize the use of that category in order to describe the way in which colonial jurisdictions were able to adapt the legal order to their specific context. ${ }^{40}$

We could say that the catholic religion, the doctrines of Ius Commune and the Castilian law were part of a hegemonic order which was enforced throughout the colonies by means of multiple institutional processes that required the intervention of authorities conditioned by local circumstances. Of course, it was not a case of foreign norms except for the original inhabitants. However, if we consider the case of the Spanish settlers and their so called Spaniards Republics we would say that they had a political identity linked to their local republics, grounded in the corporative structure of the ancient society; they also shared a set of common localized interests that they could invoke in order to adapt, transform and even resist general standards. Certain features of the pre-contemporary legal culture, with special relevance in the colonial world, contribute to this outcome: they were particularism, casuism and normative factualism (that is to say, the admitted possibility of derive a norm from factual conditions); these three cultural patterns converged necessarily in the process of localization of law. ${ }^{\mathbf{4 1}}$

38 ACHARYa (2004) 239-275.

39 Examples in Merry/Stern (2005) 387-409. See also, Merry (2006) 99-116.

40 Clavero has recently warned about the possible neocolonial intentions that may lie behind this parallelism and also in the theory of legal transplants and other related concepts, Clavero (2012) 718-729. Being aware of that, we still think that the notion of localization is useful to understand not just the colonial legal practice but some peculiar features of the legal developments after the independence processes in Latin American countries.

41 On particularism and casuism in the Spanish colonial law, see TaU Anzoátegui (1992a); on factualism in the medieval grounds of the ancient legal culture, Grossi (1996) 89. On 
If we consider, for instance, the value assigned to customary law, we could say that it was a consequence of the dynamic effects of those three cultural traits: in a good extent the normative strength of custom was derived from particularist and casuist convictions while the constant practice of the same behavior was its factual base. Solórzano Pereyra - the most famous jurist in Spanish colonial law - said that «by custom often becomes licit what is not» and that ancient custom «has the presumption that is convenient and useful». ${ }^{42}$ This last reference to what was «convenient» should be read under the hermeneutic frame of a long semantic tradition that linked what was considered appropriate by nature for each thing (convenientia rerum) with the central concept of aequitas, from which, in turn, the notions of justice and law were derived. ${ }^{43}$ Thus, different but connected notions as convenience and aequitas were valid to support a process of localization.

There was still another cultural element that contributed to localize the law. It did not come from the ground of facts but from the sky of transcendental principles of justice that, ultimately, gave normative strength to positive law. According to Catholic theology, an unjust law or any standard which could be considered against religion or natural law did not have to be obeyed. As a theologian in Lima, in 1604, stated in a commentary about some royal letters: «[...] in the things that are manifestly unjust or illegal and against the laws of God, no command of any man even if he is the King can oblige». ${ }^{\mathbf{4 4}}$

The widespread use in the Spanish colonies of the medieval formula «I obey but do not execute», as a measure to resist some Royal commands, was a good example of the convergence of both factualism and that transcendent conception of justice: a command had to be just to oblige, but to be just it had to be free from vices of obreption and subreption, that is to say, it had to be sustained in a truthfully description of facts. ${ }^{45}$ In practical terms, this convergence implied a necessary and ongoing search for consensus on what could be considered just or fair according to local circumstances, to prevent

factualism in the pre-contemporary moral language, see Macintyre (2007) 57-78. A typical case of factualism in the New World can be seen in the normative function assigned to the concept of new land as it was stressed by Mariluz Urquijo (1976) 389-402.

42 Solórzano Pereyra (1648), (1736) II, 77.

43 Grossi (1996) 180.

44 AgÍ́a (1604) 76-77.

45 Tau Anzoátegui (1992b) 69-143. 
that the positive laws, including decisions taken from the top of the royal power, were considered as unjust and then legitimately disobeyed. By reasons of aequitas it was also possible to invoke local convenience as public utility to resist legitimately a positive command.

We can see how that combination of facts, conveniences and principles was still in the background of some legal treaties of the eighteenth century when they deal with the topic of obedience. In 1748 Santayana's advised royal magistrates to not execute royal's commands from which could result an offence to the royal conscience (that is to say, to religious standards, mainly), to law (in objective sense), to public utility, or that may cause any manifest unjust injury to someone's acquired rights. Considering the discontinuous nature of the public sphere, the reference to public utility opened the door for reasons related to local convenience or to what could be considered useful in a particular place in order to disobey a royal command. At the same time, given the communitarian cultural background, the notion of acquired right could be perfectly understood as referred to local corporative privileges rather than to individual rights.

According to this, the strength of a positive command, in general terms, was conditioned by a double interpretative aperture: one derived from objective principles of justice (the royal conscience, the objective law) and other linked to the specific conditions of enforcement (public utility, acquired rights). This double opening was more functional for processes of localization considering that institutional authorities were not set as merely executive but as jurisdictional agents. ${ }^{46}$ The jurisdictional role of authorities implied necessarily an interpretative activity in which both ends must meet, connecting what was just and fair with what was convenient according to local circumstances.

Finally it should be noted that the legal order, so open, flexible and responsive to the specific conditions of local enforcement, was largely managed by authorities closely linked to local communities. If this aspect was clearly evident in the New World, it was also a pervasive feature among governments of metropolitan territories, although overshadowed by the image of a centralized state conveyed by classic historiography. Let's see, for example, how in 1800 Dou described local jurisdictions in Spain analyz- 
ing the question about the district of territorial magistrates of royal appointment (corregidores and alcaldes mayores). He said that the jurisdiction of corregidores and alcaldes mayores was limited to their city of residence and to the towns that did not have their own justices, adding that so in Castile as in Catalonia most of villages and towns had their ordinary judges «different and independent» of corregidores and alcaldes mayores, for carrying out justice. $^{47}$

On that sort of network of «independent» local justices rested the daily enforcement of a cumulative compound normative universe, composed of doctrinal and legal elements of diverse origin, shaped by practices, customs and privileges of each place. As justice and government at the local level were functions assigned to the same kind of jurisdictional authorities, judges were not only meant to determine lawsuits according to that complex normative universe, but also to keep the peace taking into account reasons of opportunity and convenience (nowadays typical of governmental institutions) in their daily task. All these elements that formed a «justice of judges not of laws» - as it has been recently suggested - played a significant role in processes of localization. ${ }^{48}$

At last, it should be also noted that talking about localization does not mean denying the hegemonic nature of the common legal culture which provided the framework of possibility for local law, or rejecting the central role of royal jurisdiction in the institutional order of the ancient regime. On the contrary, because of the hegemonic trait of its cultural and religious grounds, processes of localization were possible and necessary within the extension of the Spanish imperial order. Thus, far from being an unwanted or conflicting effect, localization processes fulfilled an essential role in the maintenance of political balances in such imperial extension. The central idea of proposing the notion of localization is to pay more attention to the weight of those cultural patterns (particularism, casuism, factualism, transcendent idea of justice, normative value of reason of convenience, etc.) that inclined the exercise of power to mediate between the general

47 «Las villas en Castilla, y en Cataluña no solo las villas sino también casi todos los lugares tienen juez ordinario, distinto e independiente del corregidor, y alcalde mayor en la administración de justicia», Dou y DE BAssols (1800) II, 134. On the colonial administration, Agüero (2008) 67-124.

Lorente Sariñena (2007). 
standards of an hegemonic culture and the local needs of different political corporations structured upon the base of deep communitarian convictions.

As a complementary category related to the topic of local law, localization denotes the dynamic processes by which different authorities, regardless of royal or local appointments, adjusted the general standards to local conditions by means of carrying out justice, governing or granting special protections and exceptions (provisiones, amparos) upon request of each community. ${ }^{49}$ In the following section we analyze some significant examples illustrating the phenomenon of localization in a peripheral context of the Spanish Monarchy as it was the former province of Tucumán and, in particular, the city of Córdoba.

\section{Local law and localization of law in the colonies}

As we have seen, due to its intrinsic characteristics, the institutional order that Spaniards brought to the New World was flexible and adaptable. This was a consequence of its own cultural background rather than a result of a special Crown policy aimed to rule her colonial domains. ${ }^{50}$ Certainly, colonial factual conditions may have often influenced in taking the inherent elasticity of the Hispanic legal culture to an extreme degree but, in turn, that flexibility played a key role in making possible the institutional organization of the New World, helping the Monarchy keep the loyalty of the colonial elites on its side almost until the fall of the Spanish Atlantic Empire.

Spanish colonization was carried out by two different institutional devices: on the one hand, territories were organized in provinces under the authority of a magistrate of royal appointment (governors, corregidores, alcaldes mayores); on the other, settlers were bound to establishing cities, that is to say republics, receiving all of them the privileges and freedoms that the Castilian law granted to municipalities. ${ }^{51}$ It was within every municipal territory where institutions and Christian political life (policía cristiana)

49 Agüero (2008) 235-275. On local law and political representation, AgüEro/Oyarzábal (2013) 263-306.

50 As it was suggested by Phelan (1960) 47-65; a general approach on this topic in TaU Anzoátegui (1992a) 83-140. For Portuguese colonies, Hespanha (2006) 59-81.

51 On the institutional organization of territories, GARRIGA (2006) 35-130; about colonial municipalities, the classic reference is BAYLE (1952). 
had place and where local law was developed by means of statutes for regulation of common goods and needs and also by local customs. It has been said that customary law had a special strength in the colonies and that the colonial law was a sort of paradise for praeter and even contra legem customary law. ${ }^{52}$

Judicial records of a bordering city like Córdoba del Tucuman give us significant testimonies about the way in which local law and customs were integrated in dynamic processes of localization. Despite the constant lack of letrados (legal professionals) in the district, litigants showed an accurate knowledge of the normative arguments that were able to support localization strategies. One of them consisted of assigning special value to "practice» that is to say the habits and styles of the local courts. As a lay defender said in 1746, «practice repeals all laws and even makes laws». Common Law doctors (Bartolo, Jaso) were quoted to uphold that statement and to conclude that the judgment given against the «evident practice» was null. ${ }^{53}$

In addition to this, special categories of the legal language like the concept of notorious crime or the formula «public voice and fame», used to qualified statements related to facts or ways of life attributed to the defendants, opened the door for introducing local prejudices and beliefs in the judicial decision making process. While the «usefulness and convenience» of each republic could be invoked to give legal effect to a number of arguments related to local needs, subjective circumstances - as having a good reputation (buena fama) - allowed to take into account more specific local conditions as is the case of social hierarchies among the members of each community. ${ }^{54}$

Judicial records show also the special strength of local customary law. In 1762, a woman claimed she was entitled to defend her husband being a fugitive, despite the positive laws that required the presence of the accused to hear his defense. She invoked the «very laudable, prescribed and well established custom in this city and its courts», according to which it was «admitted to hear the wives of an absent defendant in cases of this nature». She also recalled that «[the King] approves and declares that the customs of the cities

52 Floris Margadant (1990) 172; Barrientos Grandon (2000) 293-390; Tau Anzoátegui (2001) 182-233.

53 Archivo Histórico de la Provincia de Córdoba (hereafter AHPC), Crime, vol. 5, file 5, 1746.

54 AgüEro (2008) 344-360. 
and places are special law and that custom is even more valid when it is [more] opposed to positive law, because with it privileged force repeals the laws, according to the Common Law and doctors opinions». She proved the said custom with testimonies taken from the local courts records. At the appeal stage, The Royal Court of the distant city of Charcas supported her argument, disposing that she had the right to allege in defense of her fugitive husband. ${ }^{55}$ In doing so, the highest royal court in the region approved the value of a local custom over the positive general law showing thus that localization was not exclusively a matter of localized elite's interests but a way of normal functioning of the legal culture.

The weight of factual conditions in normative reasoning implied a constant slide of law determination to the particular context of its effective enforcement. For this reason, some institutions usually described as tools of a centralized Monarchy - like penal servitude - were also used at the services of local republics in the colonial world. A typical colonial example is the process by which New Spain settlers achieved to use forced labor (servicio personal) of Indians as a kind of punishment, despite the many royal laws that attempted to ban this practice. Faced with these prohibitions, in the mid-sixteenth century, they argued that forced labor of Indians should be permitted as a kind of penalty, because, among other reasons, in America there were «no galleys or borders or other places where they can be forced to serve» and because there was no way to control banishments (due to the features of the territory). In addition to this, they argued that Indians didn't suffer whipping penalties in the same way the Spanish did (because they lacked the same sense of honor) and that forced labor was even convenient for Indians themselves because during this time they could learn skilled trades and good manners. The Crown responded by sending a royal letter stating «for everyone in their district to do and provide what they may see as more convenient and just according to what they can and ought to do ...». ${ }^{\mathbf{5 6}}$

That decision, along with similar ones sent thereafter, was included in the compilation of royal colonial laws of 1680 , the so called Recopilación de las Leyes de Indias. The Crown had to accept that the penalties of forced labor

55 AHPC, Crime, vol. 17, file 3, 1762.

56 Zorita (1574) (1983-1984) Book VIII, title VI, law 3 $3^{\mathrm{a}}$, 355-356. 
imposed on the Indians were applied to the needs of the «republics». ${ }^{57}$ But the same kind of argument can be found in different contexts throughout the colonial world. In 1753, a prosecutor from Córdoba argued that, given the difficulties in applying penalties like galleys in the districts of the hinterland and given also the lack of «presidios», the punishment should fit the "comfort of each place» so that crimes do not rest unpunished, and added: «as it is clearly seen in this city the practice followed with the prisoners sentenced to die at the gallows, as there is no executioner or other person to take over, they are sent to be shot and after that the death body is hung from the gallows $[\ldots] \gg .{ }^{58}$

Out of the courts of justice, the normative function of local conditions played also a role in localization, particularly, when cities submitted petitions to high royal authorities claiming for a special privilege. For example, in the middle of the eighteenth century, the city of Córdoba pleaded before the Royal Council of the Indies (the highest bench of royal jurisdiction for the Indies) to get a special law to allow its judges to impose death penalties for crimes committed with a knife or any kind of dagger «regardless if the wound was mortal or not». In its petition the city argued that these kinds of crimes had been increasing in the last decade and that the usual penalties were not enough to prevent this misconduct. The Royal Council conceded the requested norm, on one condition: the local judges had to get a confirmation from the Royal Court of the district before executing the penalties. As the royal attorney pointed out before the Council, only the special local circumstances alleged by the city could authorize such harsh penalty that would be unjust in a different context. ${ }^{59}$

One special case of localization in the province of Tucuman can be considered as paradigmatic for its institutional implications. It is about the way in which the cities of the said Province, by means of petitions invoking local factual conditions and the "welfare of the republics", got a special provision from the Royal Court of the district and the Royal Council of the Indies to repeal in their territories the general rule according to which

57 Recopilación de las leyes de los Reynos de las Indias (1680, 1791) (1998), Book I, title 7, laws 8 and 10.

58 AHPC, Crime, vol. 7, file 15, 1753.

59 Agǘro (2007) 13-45. 
royal judges had to be outsiders. This rule was a general standard aimed to guarantee the impartiality of royal magistrates in the provinces. It was in the Castilian law and also in the laws for the colonies. ${ }^{\mathbf{6 0}}$ However, in different opportunities since 1584 the cities pleaded against that rule claiming that foreigner lieutenants of governors caused damages to the republics and there was no way to make them responsible after they had left the towns. In 1627, the Royal Court of Charcas decided that «Tenientes» appointed by governors had to be «vecinos» of the cities and not outsiders. In the grounds of its decision, the Court considered the conflicts experienced in the region, the «damage and inconveniences» suffered by the cities and townspeople for their «great poverty» and that those who were outsiders, «destroy and wipe out the cities as they use their posts to enrich themselves». The special provision was approved by the Royal Council in Spain in 1634. Ten year later, in a new case of conflict, and upon request of all the cities in the province of Tucuman, the Court issued a new royal provision stating that «the custom of this province to appoint, for the post of Tenientes, their own vecinos, be observed [...]». It is remarkable to note how a special privilege conceded by the Royal Court and Royal Council had become into a «custom of the province». ${ }^{\mathbf{6 1}}$

Another interesting case of localization is to be found in the way in which governors and their lieutenants of this region acquired the competence to act as judges of appeal. At first it was just a local practice justified by the huge dimension of the districts and the long distances that separated the cities from the Royal Courts. However, after a conflictive case, in 1718, the Royal Court of Charcas decided that all first instance judges of Tucuman had to give their appeals directly to that Court and not to governors or tenientes. Governors and tenientes took the case to the Council of the Indies in Spain. They argued that the said decision was against the law and against the customs of the province. Many cities supported this argument. The Cabildo of Córdoba sent a letter to the Council of the Indies expressing that it was an «immemorial custom of the region» to take the appeals to the governors, and

60 For the Castilian law González Alonso (1970) 300. For colonial law, the prohibition was sent in 1552 to New Spain, Encina (1596) (1945-1946) III, 10-11. It was included in the Recopilación de las leyes de los Reynos de las Indias (1680, 1791) (1998), book V, title II, law 45.

61 The case is analyzed in AgüEro/Oyarzábal (2013). 
that it must be maintained given the poverty of most of the litigants that made it impossible for them to reach the distant Court of district. After consulting the case with the Viceroy of Peru, in 1751 the Council of the Indies decided to maintain the competence of governors to act as judges of appeal in their districts. ${ }^{\mathbf{6 2}}$

The last two cases show how strategies of localization could affect significant standards of the institutional structure in the peripheral colonial context. Of course, much of the Bourbons reforms, in the late eighteen century, were addressed to change those traditional practices. The new Governor Intendants did not act as judge of appeal. But as they were also deprived of the faculty of delegating their jurisdiction in lieutenants, most of the cities remained ruled by their own local judges except the capital of Intendance. In this particular topic, it can be said that the reforms strengthened the localization of justice administration and the sense of self government of the local elites. ${ }^{63}$

Anyhow, beyond the discussion about the Bourbons reforms, what they did not change at all was that fundamental cultural conceptions according to which justice was to serve the welfare of the republics and that it had to be adjusted to what could be regarded as more suitable for each place. ${ }^{64}$ Due to this kind of reasoning, during the Intendant period in Córdoba penalties of forced labor applied to public works in the city had a marked increase. Upon the argument of local needs, a new kind of punishment, consisting in banishment with the obligation for the convict and his family to settle in a village close to the frontiers was also introduced. In the same way, a new measure of social control was implemented, by local statute, consisting of a compulsory labor relationship with a landowner (obligación de conchavo) imposed on anyone who had been subjected to criminal prosecution (as a condition to be released after serving the penalty) or to anyone who, not being able to justify an independent way of living, could be qualified as a bum. ${ }^{65}$

Strategies of localization were even useful in that period to resist the attempt of control undertook by the new Royal Court of Buenos Aires in

62 LeVagGi (1984) 192-193.

63 LeVAGgi (1991) 375.

64 On Bourbons reforms in colonial justice administration, GarRIGA (2002) 781-821.

65 González de Martínez (1994), Tau Anzoátegui (2004) 347-479. 
1785. That year the Royal Court decided to limit the punishment power of the local judges of the Viceroyalty in order to prevent abuses in the exercise of criminal justice. A general rule was passed requiring that sentences imposing penalties of whipping or other afflictive punishments had to be confirmed in the Court of Buenos Aires before being executed. The cities soon claimed against this measure. Local judges from Córdoba, for example, argued that if they had to do so in every case, most of the crimes would remain unpunished because of the long time that it would take the confirmation proceedings and the lack of security in the local jail. The Intendant of Córdoba supported these arguments and asked the Court to authorize the local judges to execute sentences imposing penalties of up to 25 or 30 lashes and of one or two years of forced labor without any formal proceeding for the most common crimes, like cattle rustling, that caused great damage to local landowners. The request was justified in the «circumstances of the country», "the impossibility of following a complete formal proceeding with so many cases of thefts of horses, cattle stealing and the like, commonly committed by the plebe and vile people [...]». The Intendant also mentioned the characteristics of the territory that provided facilities for fugitives and recalled the «universal clamor of the landowners for punishing thefts of all kinds of cattle they suffer». The Court admitted the arguments, and granted an exception to authorize the local judges of Cordoba to execute, without the need to carry out the confirmation proceeding, penalties of 25 lashes and four months of forced labor for crimes of theft and other misdemeanors, «according to the circumstances of the accused and his crime» under the supervision of the Intendant. The same was accepted for other cities of the Viceroyalty. ${ }^{66}$

Looking at the long transition period that began after the crisis of the colonial order, it would be possible to say that processes of localization reached it most remarkable expression during the first half of the $19^{\text {th }}$ century in the Rio de la Plata region, particularly after the decade of 1820 when the old cities shifted their institutional nature from municipalities into sovereign republics upon the base of their foundational privileges and their long lasting sense of self government. Free from any external jurisdictional control, local elites enacted, within their territories, mechanism of expedi- 
tious justice potentiating solutions that had begun as local law during the last decades of colonial era. Furthermore, due to the long lasting experience of localization, the ancient local jurisdictions became a fundamental element during the constituent conventions that took place in 1853 and 1860, shaping a peculiar feature of the Argentinean federalism. ${ }^{67}$

\section{Final reflections: local law and localization}

To conclude these brief reflections, we would like to highlight some analytical virtues that the notion of localization can offer as a complementary category for the historical study of local law. Firstly, as it is meant to denote a dynamic process, the notion of localization avoids the risk of incurring in the anachronism of connecting the static concept of local law with our current comprehension of the normative order as pyramidal scheme of hierarchy.

Second, though related, while the idea of localization does not fit with the current theory of sources of the law (often retro-projected to the past), it is useful to think about the local law beyond the restricted limits provided by the customary or statutory forms of law. In other words, the notion of localization has not the implicit semantic load that tends to link the local law to municipal ordinances according to the current classification of the territorial validity dominions (city, province, state). On the contrary, localization is functional to describe processes of determination of law regardless the formal hierarchy of norms, highlighting the complementarity and subsidiarity relationship between the general order and the local praxis, stressing the normative weight of contextual circumstances. These had a special incidence in a legal culture that identified the legitimate exercise of power with different forms of jurisdiction.

Finally, if localization of law played a significant role in helping the Monarchy keep the loyalty of the colonial elites on its side almost until the fall of the Spanish Atlantic Empire, it provides, in turn, an heuristic key for the comprehension of the legal developments after the said falling when, on both sides of the Hispanic Atlantic world, local-centered tendencies - using in a wide sense the expression suggested by Van Young for Mexico - 
became dominant in the struggles for the exercise of political powers and territorial representation, ${ }^{\mathbf{6 8}}$ hindering the (sometimes rhetoric) goal of establishing a national legal order based on a general and abstract conception of law.

\section{Bibliography}

Acharya, Amitav (2004), How Ideas Spread: Whose Norms Matter? Norm Localization and Institutional Change in Asian Regionalism, in: International Organization, The MIT Press 58, n. 2, 239-275

Agía, Miguel (1604), Tratado que contiene tres pareceres graves en Derecho sobre justificación de una Cedula Real que trata del servicio personal, y repartimientos de indios, Lima: Antonio Ricardo

Agüero, Alejandro (2007), Ley penal y cultura jurisdiccional. A propósito de una Real Cédula sobre armas cortas y su aplicación en Córdoba del Tucumán, segunda mitad del siglo XVIII, in: Revista de Historia del Derecho 35, 13-45

Agüero, Alejandro (2008), Castigar y perdonar cuando conviene a la república. La justicia penal de Córdoba, siglos XVII y XVIII, Madrid: Centro de Estudios Políticos y Constitucionales

Agüero, Alejandro (2010), La justicia penal en tiempos de transición. La república de Córdoba, 1785-1850, in: Garriga, Carlos (coord.), Historia y constitución. Trayectos del constitucionalismo hispano, México: Instituto Mora, 267-305

Agüero, Alejandro (2011), Tradición jurídica y derecho local en época constitucional: El «Reglamento para la Administración de justicia y policía en la campaña» de Córdoba, 1856, in: Revista de Historia del Derecho 41, 1-43 (available at: http://www.scielo.org.ar/scielo.php?script=sci_arttext\&pid=S1853$17842011000100001 \& \operatorname{lng}=$ es\&nrm=iso)

Agüero, Alejandro (2012), On justice and home rule tradition in the Spanish colonial order. Criminal justice and self government in Cordoba del Tucuman, in: Quaderni Fiorentini per la storia del pensiero giuridico moderno 41, 173-221

Agüero, Alejandro (2013), Ciudad y poder político en Antiguo Régimen. La tradición castellana, in: Tau Anzoátegui, Víctor, Alejandro Agüero (coords.), 121-184

Agüero, Alejandro, Maria Cecilia Oyarzábal (2013), Derecho local y representación provincial. Reflexiones a partir del memorial presentado por la Provincia

68 VAN Young (2010). About the imprints of the ancient traditions on the constitutional discussion in the Hispanic Atlantic world, Lorente/ Portillo (2011). 
de Tucumán al Consejo de Indias, 1631-1633, in: TAu Anzoátegui, Víctor, Alejandro Agüero (coords.) (2013), 263-306

Arevalillo Doval, Juan José (2000), La localización: concepto, nuevas tecnologías y requisitos del nuevo traductor de informática, working paper in: IVth Encuentros Alcalaínos de Traducción, Universidad de Alcalá de Henares (available at: http://bscw.rediris.es/pub/bscw.cgi/59200?client_size=1280x605)

Barrientos Grandon, Javier (2000), Historia del Derecho Indiano. Del descubrimiento colombino a la codificación. I. lus Commune - Ius Propium en las Indias Occidentales, Roma: Il Cigno Galileo Galilei

Bayle, Constantino (1952), Los cabildos seculares en la América española, Madrid: Sapientia

Benton, Lauren (2002), Law and colonial cultures. Legal Regimes in World History: 1400-1900, Cambridge: Cambridge University Press

Bravo Lira, Bernardino (1988), El Derecho Indiano y sus raíces europeas: Derecho Común y propio de Castilla, in: Anuario de Historia del Derecho Español XCVIII, 5-80

Burns, Robert (ed.) (2001), The Medieval Church, Volume 1: The World of Clerics and Laymen, English translation of Las Siete Partidas by Samuel Parsons Scott, Philadelphia: University of Pennsylvania Press

Castillo de Bovadilla, Jerónimo (1597, 1704) (1978), Política para corregidores y señores de vasallos, en tiempo de paz, y de guerra, Amberes, facsimilar ed., Madrid: Instituto de Estudios de Administración Local

Clavero, Bartolomé (1978), La disputa del método en las postrimerías de una sociedad: 1789-1808, in: Anuario de Historia del Derecho Español 48, 307-334

Clavero, Bartolomé (2012), Gracia y Derecho entre localización, recepción y globalización (lectura coral de las vísperas constitucionales de António Hespanha), in: Quaderni Fiorentini per la storia del pensiero giuridico moderno 41, 675-793

Donini, Massimo (2003), ¿Una nueva edad media penal? Lo viejo y lo nuevo en la expansión del derecho penal económico, in: Nuevo Foro Penal, núm. 65, 92-119

Dou y de Bassols, Ramón Lázaro (1800), Instituciones de Derecho Público de España, Madrid: Officina de Don Benito García y Compañía

Encina, Diego De (1596) (1945-1946), Cedulario Indiano, facsimilar ed., Madrid: Cultura Hispánica

Floris Margadant, Guillermo (1990), La consuetudine contra legem en el Derecho Indiano a la luz del Ius Commune (Análisis del pensamiento de Francisco Carrazco del Saz, jurista indiano, sobre este tema), in: Anuario de Historia del Derecho Mexicano 2, México: Universidad Nacional Autónoma de MéxicoInstituto de Investigaciones Jurídicas, 169-188

Fortea Pérez, José (1991), Poder real y poder municipal en Castilla en el siglo XVI, in: Pastor Reyna et al., Estructuras y formas de poder en la historia, Salamanca: Universidad de Salamanca, 117-142 
Garriga, Carlos (2002), Los límites del Reformismo Borbónico: a propósito de la administración de la justicia en Indias, in: Barrios Pintado, Feliciano (ed.), Derecho y administración pública en las Indias hispánicas: actas del XII congreso internacional de historia del derecho indiano, Cuenca: Cortes de Castilla La Mancha - Universidad de Castilla La Mancha, I, 781-821

Garriga, Carlos (2004), Orden jurídico y poder político en el Antiguo Régimen, in: ISTOR - Revista de Historia Internacional, Año IV, número 16 (primavera de 2004), 13-44

Garriga, Carlos (2006), Patrias criollas, plazas militares: sobre la América de Carlos IV, in: Martiré, Eduardo (coord.), La América de Carlos IV, Buenos Aires: Instituto de Investigaciones de Historia del Derecho, 35-130

Garriga, Carlos, Andréa Slemian (2013), «Em trajes brasileiros»: justiça e constituição na América ibérica (c. 1750-1850), in: Revista de História São Paulo, $\mathrm{n}^{\mathrm{o}} 169,181-221$

González Alonso, Benjamín (1970), El Corregidor Castellano (1348-1808), Madrid: Instituto de Estudios Administrativos

González de Martínez, Marcela (1994), Control social en Córdoba. La papeleta de Chonchabo 1772-1892 (Documentos para su estudio), Córdoba: Centro de Estudios Históricos

Grossi, Paolo (1996), El orden jurídico medieval, Madrid: Marcial Pons

Hespanha, António Manuel (1986), A historiografía jurídico-institucional e a "morte do estado", in: Anuario de Filosofía del Derecho, Madrid: Instituto Nacional de Estudios Jurídicos, III, 191-227

Hespanha, António Manuel (1993), La gracia del derecho. Economía de la cultura en la Edad Moderna, Madrid: Centro de Estudios Constitucionales

Hespanha, António Manuel (2006), Porque è que existe e em que è que consiste um direito colonial brasileiro, in: Quaderni Fiorentini per la storia del pensiero giuridico moderno 35, 59-81

Hespanha, António Manuel (2007), Depois do Leviathan, in: Almanck brasiliense 5, 55-66 (available at: www.almanack.usp.br)

Kantorowicz, Ennst (1985), Los dos cuerpos del Rey. Un estudio de teología política medieval, Madrid: Alianza

Kelsen, Hans (1982) Teoría pura del derecho, México: Universidad Nacional Autónoma de México

Lacchè, Luigi, Massimo Meccarelli (2012), Introduzione. Storia del diritto come storia della giustizia: materiali per una riflessione metodologica, in: LACCHÈ, Luigi, Massimo Meccarelli (eds.), Storia della giustizia e storia del diritto. Prospettive europea di ricerca, Macerata: Edizioni Università di Macerata

Lempérière, Annick (2004), Entre Dieu et le Roi la République, Mexico, XVI ${ }^{\mathrm{e}}$ $\mathrm{XIX}^{\mathrm{e}}$ siècles, Paris: Les Belles Lettres

Levaggi, Abelardo (1984), El recurso de apelación en el Derecho Indiano, in: Revista de Historia del Derecho 12, 171-198

Levaggi, Abelardo (1991), Elección bianual y confirmación de los alcaldes ordinarios bajo la Ordenanza de Intendentes del Río de la Plata, in: IX Congreso del 
Instituto Internacional de Historia del Derecho Indiano, Madrid: Editorial de la Universidad Complutense, II, 351-380

López, Gregorio (1555) (1985), Las Siete Partidas glosadas por el Licenciado Gregorio López (1555), facs. ed., Madrid: Boletín Oficial del Estado

Lorente Sariñena, Marta (coord.) (2007), De la justicia de jueces a la justicia de leyes: Hacia la España de 1870, Madrid: Consejo General del Poder Judicial

Lorente Sariñena, Marta, José Maria Portillo (2011) (dirs.), El momento gaditano. La Constitución en el orbe hispánico (1808-1826), Madrid: Cortes Generales

Macintyre, Alasdair (2007), After Virtue. A Study in Moral Theory, Notre Dame: University of Notre Dame Press

Mackay, Angus (1985), La España de la Edad Media desde la frontera hasta el imperio 1000-1500, Madrid: Cátedra

Mannori, Luca (1990), Per una 'preistoria' della funzione amministrativa. Cultura giuridica e attività dei pubblici apparati nell'età del tardo diritto comune, in: Quaderni Fiorentini per la storia del pensiero giuridico moderno 19, 323-504

Mariluz Urquijo, José María (1952), La Real Audiencia de Buenos Aires y la administración de justicia en lo criminal en el interior del Virreinato, in: Primer Congreso de Historia de los Pueblos de la Provincia de Buenos Aires, La Plata, 271-291

Mariluz Urquijo, José María (1976), El concepto de tierra nueva en la fundamentación de la peculiaridad indiana, in: IV Congreso Internacional de Historia del Derecho Indiano, México: Universidad Nacional Autónoma de México, 389-402

Merry, Sally Engle (2006), Anthropology and International Law, in: Annual Review of Anthropology 35, 99-116

Merry, Sally Engle, Rachel E. Stern (2005), The Female Inheritance Movement in Hong Kong: Theorizing the Local/Global Interface, in: Current Anthropology, The University of Chicago Press - Wenner-Gren Foundation for Anthropological Research 46, n. 3, 387-409 (available at: http://dx.doi.org/10.1086/ 428800)

Nicolini, Ugo (1982), Autonomia e diritto proprio nelle città italiane del Medio Evo, in: Horn, Norbert (Hg.), Europäisches Rechtsdenken in Geschichte und Gegenwart. Festschrift für Helmut Coing zum 70. Geburtstag, München: C. H. Beck, I, 249-267

Phelan, John Leddy (1960), Authority and Flexibility in the Spanish Imperial Bureaucracy, in: Administrative Science Quarterly 5, n. 1, 47-65 (available at: http://dx.doi.org/10.2307/2390824)

Recopilación de las leyes de los Reynos de las Indias (1680, 1791) (1998), facsimilar ed. Madrid: Centro de Estudios Políticos y Constitucionales - Boletín Oficial del Estado

Recopilación de las leyes destos reynos (1567) (1640) Madrid, Printed by Catalina de Barrio Angulo y Diego Diaz de la Carrera 
Rojas, Beatriz (coord.) (2007), Cuerpo político y pluralidad de derechos. Los privilegios de las corporaciones novohispanas, México: CIDE

Sanponts y Barba, Ignacio et al. (1843-1844) (eds.), Las Siete Partidas del sabio Rey don Alfonso el IX (sic), con las variantes de más interés y con las glosas del Lic. Gregorio López, del Consejo Real de Indias de S. M. vertidas al castellano y extensamente adicionada con nuevas notas y comentarios y unas tablas sinópticas comparativas, sobre la legislación española, antigua y moderna, hasta su actual estado, Barcelona: Imprenta de Antonio Bergnes

Sassen, Saskia (2006), Territory, Authority, Rights: From Medieval to Global Assemblages, Princeton: Princeton University Press

Schaub, Jean-Frédéric (1998), El pasado republicano del espacio público, in: Guerra, François-Xavier et al., Los espacios públicos en Iberoamérica. Ambigüedades y problemas. Siglos XVIII-XIX, México: Centro Francés de Estudios Mexicanos y Centroamericanos y Fondo de Cultura Económica, 27-53

Solórzano Pereyra, Juan de (1648) (1736), Política Indiana, Madrid: Mateo Sacristán

Sousa Santos, Boaventura de (1998), La globalización del derecho. Los nuevos caminos de la regulación y la emancipación, Bogotá: Universidad Nacional de Colombia - Facultad de Derecho, Ciencias Políticas y Sociales, Instituto Latinoamericano de Servicios Legales Alternativos

TAu Anzoátegui, Víctor (1992a), Casuismo y sistema. Indagación histórica sobre el espíritu del Derecho Indiano, Buenos Aires: Instituto de Investigaciones de Historia del Derecho

Tau Anzoátegui, Víctor (1992b), La ley en América Hispana. Del descubrimiento a la emancipación, Buenos Aires: Instituto de Investigaciones de Historia del Derecho

Tau Anzoátegui, Víctor (2001), El poder de la costumbre. Estudios sobre el Derecho Consuetudinario en América Hispana, Buenos Aires: Instituto de Investigaciones de Historia del Derecho

Tau Anzoátegui, Víctor (2004), Los Bandos de buen gobierno del Río de la Plata, Tucumán y cuyo en la época hispánica, Buenos Aires: Instituto de Investigaciones de Historia del Derecho

Tau Anzoátegui, Víctor (2005), La configuración del Derecho indiano provincial y local. Problemas terminológicos e históricos, in: Torres Aguilar, Manuel (ed.) (2005), Actas del XV Congreso del Instituto Internacional de Historia del Derecho Indiano, Córdoba: Diputación de Córdoba, Universidad de Córdoba, I, 231-253

Tau Anzoátegui, Víctor, Alejandro Agüero (2013) (coords.), El derecho local en la periferia de la Monarquía. Río de la Plata, Tucumán y Cuyo, siglos XVI-XVIII, Buenos Aires: Instituto de Investigaciones de Historia del Derecho

Vallejo, Jesús (1992), Ruda equidad, ley consumada. Concepción de la potestad normativa (1250-1350), Madrid: Centro de Estudios Constitucionales 
Vallejo, Jesús (2009), El cáliz de plata: Articulación de órdenes jurídicos en la jurisprudencia del ius commune, in: Revista de Historia del Derecho, julio/ diciembre, 38, 1-13

VAN Young, Eric (2010), El momento antimoderno: localismo e insurgencia en México, 1810-1821, in: AnNino, Antonio (coord.) (2010), La revolución novohispana, 1808-1821, México: Fondo de Cultura Económica, 221-292

Wolters, Oliver Williams (1982), History, Culture and Region in Southeast Asian Perspectives, Singapore: Institute of Southeast Asian Studies

Zarka, Yves (dir.) (2007), Monarchie et république au XVIIe siècle, Paris: Presses Universitaires de France

Zorita, Alonso de (1574) (1983-1984), Leyes y ordenanzas reales de las indias del mar oceano por las cuales primeramente se han de librar todos los pleitos civiles y criminales de aquellas partes y lo que por ellas no estuviere determinado se ha de librar por las leyes y ordenanzas de los reinos de Castilla, México: Secretaría de Hacienda y Crédito Público 

Marta Lorente Sariñena

\title{
Uti possidetis, ita domini eritis
}

\author{
International Law and the Historiography of the Territory
}

L'espace qui apparaît aujourd'hui à l'horizon de nos soucis, de notre théorie, de nos systèmes n'est pas une innovation; l'espace lui-même, dans l'expérience occidentale, a une histoire, et il n'est pas possible de méconnaître cet entrecroisement fatal du temps avec l'espace.

Michel Foucault ${ }^{1}$

\section{Chinese Histories}

Covering an area of approximately 3.5 million square kilometres, the South China Sea bathes the shores of several countries (Brunei, China, Philippines, Indonesia, Malaysia, Taiwan and Vietnam), and contains hundreds of small islands, atolls, keys, reefs and sandbanks, many of which are either underwater at high tide or remain permanently submerged. The mere mention of these waters will bring back memories to those of us who were avid readers of the 'Pirates of Malaysia' series in our youth. Today, however, the romantic role of the adventurers imagined by Salgari have been replaced by the most unromantic of coastline states that are embroiled in a dispute over a region of crucial geostrategic importance, with a high concentration of hydrocarbon reserves and abundant fishing resources. ${ }^{2}$ The People's Republic of China undoubtedly plays the lead role in this highly complex and conflictridden plot, ${ }^{3}$ whose most violent episodes reach the world news headlines. ${ }^{4}$

1 Foucault (1984) 46-49.

2 Samuels (1982); Johnston/Valencia (1991).

3 Yee (2011) 165-19.

4 Valencia (2007) 127-167; Valencia (2010) 8-17. 
I will emphasize at this point one particular aspect of the contention in the China Seas, namely: ${ }^{5}$ Beijing claims a major part of the territory under dispute with arguments unearthed from documents thousands of years old. ${ }^{6}$ Indeed, simply by visiting some of the People's Republic of China's official sites we can see that the Chinese Government fully subscribes the opinions of certain scholars, ${ }^{7}$ who hold that the fact that China was the first to discover, explore and exploit the area, «has naturally led the Chinese rulers and people to believe that the South China Sea Islands were part of China [...] from the Xia Dynasty $\left(21^{\text {st }}-16^{\text {th }}\right.$ centuries B. C. $)$ to the Qing Dynasty (1644-1911)». ${ }^{8}$

The People's Republic maintains similar positions in its dispute with Japan, and, to a lesser degree, with Taiwan, over the archipelago known as Diaoyu, Tiaoyutai or Senkaku. ${ }^{9}$ The latter, composed of five islands and three rocky outcrops, is located on the edge of the Asian continental platform in the East China Sea. ${ }^{\mathbf{1 0}}$ According to Japan, after inspections carried out from 1885 showed the islands to be uninhabited, it acquired territorial sovereignty over the archipelago in compliance with current international law (occupation of terra nullius), when Japan added it to its territory after claiming victory in the Sino-Japanese War (Treaty of Shimonoseki, 1895). ${ }^{\mathbf{1 1}}$ Conversely, the People's Republic claims that the islands were unlawfully usurped on that date, as they had belonged to Chinese territory since ancient times. The islands, maintains the Chinese Government, were discovered, named, and exploited by Chinese subjects, and later recognized by imperial envoys and annexed during the Ming Dynasty, allowing them to be placed under the jurisdiction of the local government of Taiwan during the Qing Dynasty, in which situation they remained until they were finally lost. ${ }^{\mathbf{1 2}}$

5 YeE (2011) 165-19.

6 Dzurek (1996). It must be pointed out that the People's Republic claims not only the Spratly archipelago, but also the isles of Pratas, Paracel, and the Macclesfield Bank.

7 Historical Evidence To Support China's Sovereignty over Nansha Islands (available at: http://www.fmprc.gov.cn/eng/topics/3754/t19231.htm.).

8 SHEN (2002). The official site mentioned in the foregoing note contains the works of this author.

9 International Crisis Group (2013).

10 SHAw (1999).

11 An official version of the subsequent history of the archipelago can be followed at: http://www.mofa.go.jp/region/asia-paci/senkaku/.

12 http://dk.china-embassy.org/eng/ztbd/dydwt/t1036399.htm. 
The reader may wonder how the disputes over the China Seas can be related to the principle of international law known as uti possidetis referred to in the title of this paper. The answer is fairly simple given that the history of the territory plays an equally important role in the disputes over the China Seas as in those in which the principle of uti possidetis is invoked. In effect, the argument wielded by the Chinese government, according to which the historical precedent must be taken into account when determining states' territorial rights, shares the logic of the principle of the international law, according to which the new states formed through gaining their independence retain the borders they had as colonial demarcations. ${ }^{13}$

This link will seem rather far-fetched, but we should remember a couple of well-known issues that contribute to giving it a more solid grounding. Indeed, the loss of Eurocentricity in historiography, ${ }^{14}$ and the progressive universalization of society and international law, ${ }^{15}$ have contributed in no small measure to multiplying the number of histories of the territory that are susceptible of being used in border conflicts. Nevertheless, it must be highlighted that practically all of them follow the common pattern of 'history to serve the purposes' of an eminently European invention, the Nation-State, which in turn has permanently marked the nature of international law from its origins up to our time. I will leave for later the analysis of the heavily nationalistic Latin American histories that were and are used for the sake of implementing the uti possidetis, examining first the equally nationalistic claims made by China. ${ }^{16}$

International Relations experts have stated that the 'historical truths' on which the People's Republic's exterior policy with regard to what it considers 'its Seas' is grounded, can only be explained in terms of internal affairs. ${ }^{17}$ Never Forget National Humiliation, for example, is the significant title of a study by Z. Wang, who establishes a link between this policy and the strengthening of Han nationalism, ${ }^{\mathbf{1 8}}$ which was reformulated during the

13 Nesi (1996).

14 Chakrabarty (2008). More concretely, Koskenniemi (2011); Fassbender/Peters (2012).

15 Remiro Brotóns (1999) 11-58.

16 Anderson (2001).

17 Jie (1994) 893-903.

18 Hays Gries (2004). 
educational and propagandistic campaigns that commenced in the 1990s. According to Wang, one central aspect of those campaigns was the emergence of a new historical narrative that, having replaced class struggles with patriotic conflicts, purports to recover a glorious past in order to reactivate the country. ${ }^{19}$ I do not know whether or not this narrative has made use of any of the traditional categories from China's multi-millenarian culture, ${ }^{20}$ but clearly one of the causes/effects of the current territorial claims originates from the extremely popular and nationalist maxim regarding the 'century of humiliation' (1842-1949), ${ }^{21}$ during which China was forced to sign the famous unequal treaties. ${ }^{22}$ It is no coincidence, therefore, that riding on the tail of the rising star in the international scene that is the People's Republic, some of its leaders should affirm that the historical stages that have elapsed from the signature of the Treaty of Nanking (1842) to the present can be given the following highly significant labels: «humiliation, restoration and, finally, domination». ${ }^{23}$ Exaggerating but a little, it could be said that by excluding those humiliating hundred years from the order of time, China becomes connected to its extraordinary global past, ${ }^{24}$ which in turn gives legitimacy to the People's Republic's expansionist policies regarding what it considers to have been its seas. ${ }^{25}$ As might be expected, this narrative prompted internal support as much as it aroused external criticism. ${ }^{26}$ However, I will set out exclusively to give a very brief summary of the criticism questioning the validity of the historical sources used by the Chinese Government.

First, it has been said that if any specific aspect is highlighted in the historical documentation, it is precisely the disparity between past and present territorial perceptions. Indeed, just as the Chinese historical cartography barely coincides with that of today, ${ }^{27}$ the Record of Curiosities (Yiwu

19 WANG (2012).

20 Granet (2013); Hespanha (1993) 275-293; Hui (2006).

21 De Vel-Palumbo (2008).

22 WANG (2005).

23 Colombani (2012).

24 Hobson (2006).

25 LeVathes (1997).

26 WANG (2012).

27 Harley/Woodward (1994). 
Zhi) published during the Eastern Han Dynasty (23-220), or the travels the famous $15^{\text {th }}$ century navigator Cheng Ho, contain no information that is of any use in solving current border conflicts. ${ }^{28}$ Secondly, China's claims have likewise been questioned on account of their historical-legal aspects, as some authors hold the opinion that the Chinese Emperor was «King of his people, not of his people's lands», especially with regard to vassal kingdoms such as Vietnam and Siam. Finally, it is said that what the People's Republic really wants is to bring back a specific version of the Monroe doctrine in the Pacific, which would allow China to use titles that do not currently form part of international law (discovery). ${ }^{29}$

I believe that any neutral observer would accept the above arguments without much thought. But I also believe that it will not be amiss to recall certain data in order to compare these highly condensed Chinese histories against those that have been used for implementing the uti possidetis principle in the space that saw its birth: Latin America. ${ }^{30}$ Precisely in this sense, historical documentation on the territory in the form of maps, lists, descriptions or titles, which is still used in many Latin American conflicts, poses problems resembling those arising in the case of China. ${ }^{31}$ Something similar could be said of what I have called the historical-legal aspects of the power exercised by the Celestial Empire over the territory, as they hardly differ from the pre-modern perception of space that pervaded European legal culture practically up to the end of the $18^{\text {th }}$ century. ${ }^{32}$ We can also recall that, whereas discovery is considered an 'inchoate title' since the famous arbitral award rendered by Max Hubert in the case of the island of Palmas (1928), ${ }^{33}$ the fact is that it served to justify «the great land-grab by European powers of non-European soil», ${ }^{34}$ in which incidentally the roots of the uti possidetis

28 SHEN (1997) 1-75.

29 Jennings (1963).

30 Nesi (1996).

31 The problem, without doubt, is general (Kaplan [2013]), therefore the courts must work to resolve it over and over again in the case of border conflicts (Álvarez Jiménez [2011] 177-211). On how border disputes are aggravated in Latin America owing to the imprecision of maps, cfr. BRUCE ST. JoHn (1998-1999).

32 Hespanha (1993a).

33 Island of Palmas case (2006).

34 Sснмітт (1979) 143-144. 
doctrine are sunk. ${ }^{35}$ And, to finish: it escapes no one that it was the most distinguished American internationalists who sentenced, early in the $20^{\text {th }}$ century, that the uti possidetis doctrine originated directly from the expansion of the Monroe doctrine in Latin America. ${ }^{36}$

But let us forget these distasteful similarities for the time. The fact is that provided it is agreed that the territory is a legal and political notion rather than geographical, ${ }^{37}$ no continuity can be established between the space governed by the Celestial Empire and that belonging today to the People's Republic. Indeed, history not only fails to provide a solution to the dispute over the China Seas but further complicates it, ${ }^{38}$ if only because 'the past is a foreign country' regarding which countless interpretations are possible. ${ }^{39} \mathrm{I}$ have no wish to add a further reflection to the trove of arguments bearing on the impossibility of recovering the past; to the contrary, my aim herein is merely to articulate the following research proposal: the study, both of the uti possidetis principle in itself and of the documentation generated by its implementation, has the virtue of delimiting a field that is particularly relevant to this historical-juridical reflection on the 'temporal dimensions and geographical ambits' inspired by this publication. In order to provide a foundation for this concrete proposal, this paper presents (II) a brief overview of the historiography, and (III) a reflection on the historical notions of time and space that were important to the Latin American territorial disputes.

35 Garriga (2006) 35-130.

36 See infra.

37 Foucault (1976) 71-85.

38 As expected, several coastal states have responded to Chinese claims with other interpretations of the history of the disputed territory. For example, the government of Vietnam affirms: «Vietnam's case is that it has maintained effective occupation of the two archipelagos at least since the $17^{\text {th }}$ century when they were not under the sovereignty of any country and the Vietnamese State has exercised effectively, continuously and peacefully its sovereignty over the two archipelagos until the time when they were invaded by the Chinese armed forces», Ministry of Foreign Affairs, Socialist Republic of Vietnam, The Hoang Sa (Paracel) and Truong Sa (Spratly) Archipelagos and International Law, Hanoi, $1988,4$.

39 Lowenthal (1998). 


\section{American origins of the uti possidetis. A brief, critical, and no doubt incomplete, "state of the question"}

\subsection{International Law and Historiography of the territory}

It is often said that the uti possidetis doctrine is one of the most valuable Latin American contributions to international law. ${ }^{40}$ This is why scholars are wont to reproduce paragraphs from a famous sentence passed down by the International Court of justice in the Burkina Faso/Republic of Mali case, of which the following is worth noting:

«In these circumstances, the Chamber cannot disregard the principle of uti possidetis juris, the application of which gives rise to this respect for intangibility of frontiers. It emphasizes the general scope of the principle in matters of decolonization and its exceptional importance for the African continent, including the two Parties to this case. Although this principle was invoked for the first time in Spanish America, it is not a rule pertaining solely to one specific system of international law. It is a principle of general scope, logically connected with the phenomenon of the obtaining of independence, wherever it occurs. Its obvious purpose is to prevent the independence and stability of new States being endangered by fratricidal struggles provoked by the challenging of frontiers following the withdrawal of the administering power. The fact that the new African States have respected the territorial status quo which existed when they obtained independence must therefore be seen not as a mere practice but as the application in Africa of a rule of general scope which is firmly established in matters of decolonization; and the Chamber does not find it necessary to demonstrate this for the purposes of the case». ${ }^{\mathbf{4 1}}$

It is evident that in this 'rule applicable to the case' the Court raised to the condition of general principle a practice that, having emerged in Latin America in the $1800 \mathrm{~s},{ }^{42}$ not only reappeared in Africa during the second half of the $20^{\text {th }}$ century, ${ }^{43}$ but has also spread across the globe in the last

40 KoHen (2001) 57-77; SÁNCHez Rodríguez (1993) 93-115.

41 Case concerning the frontier dispute (Burkina Faso/Republic of Mali). Judgment of 22 December 1986. (available at: http://www.icj-cij.org/docket/index.php?sum=359\& $\mathrm{p} 1=3 \& \mathrm{p} 2=3 \&$ case $=698 \mathrm{p} 3=5)$.

42 Durán Bachler (1972).

43 Under the principle of intangibility of frontiers, recognized by Resolution AGH/Res. 16 (1) of El Cairo dated 21 July 1964, the Organization of African Unity repeated the previous Latin American experience: the administrative limits of a given colonial empire became international borders, and the borders between colonies of different metropolis constituted the borders of recently independent states. 
decades. ${ }^{44}$ The current state of affairs has obliged experts to certify the resurrection of the venerable Latin American doctrine of uti possidetis, which seemed to have lost effectiveness owing to the decline in border conflicts over the last two hundred years. ${ }^{45}$

I am aware that both the analysis of the causes and consequences of acknowledging uti possidetis as a principle of international law, and the assessment of the arguments in favour and against it that are discussed these days, are of greater interest than the history of the principle or the historiography generated by its implementation. Nevertheless, recent publications have shown that history is not only complementary but also necessary to international law. ${ }^{46}$ So, it can be stated that the Court 'standardized' the history of the principle since did not question whether uti possidetis had always been a principle of international law, that is, destined exclusively for use by states. ${ }^{47} \mathrm{I}$ shall return to this issue later; however, we have to admit that writing history is not listed among the Court's responsibilities.

By and large, the court was not far wrong in pinpointing the rule's origins in the newly emancipated regions of America. ${ }^{48}$ As long as it was not identified with a generic and timeless defence of the statu quo, ${ }^{49}$ the uti possidetis principle remained unrelated to the Roman interdiction that prohibited altering the possessory condition provided the latter was not gained through violence, clandestinity or a precarious title (D. $43,17,1) .{ }^{50}$ It is true that some authors maintain the opposite, ${ }^{51}$ but I do not think it fitting to insist on the famous dilemma that, with its impact on the notions of continuity/ discontinuity, has marked the debate on legal history over the last decades. ${ }^{52}$ Another entirely different matter is to analyse and assess the nature of internationalism, a legal discipline which has used and abused arguments taken

44 Terret (2000).

45 Durán Bachler (1989) 490; Sánchez Rodríguez (1988) 121-152.

46 Castellino/Allen (2003).

47 Shaw (1996) 75-154.

48 However, certain authors affirm that the origin of the principle must be the Treaty of Madrid of 1750. Cfr. Roux (2001) 515-516.

49 Gaudemet (1996) v. 1, 37-46. In any case, the expression had a military sense: «Según lo que poseéis [ahora]; actualmente indica que los beligerantes conservan los territorios ocupados hasta el momento en que cesan las hostilidades». Segura Munguía (2007) 146.

50 KoHen (1997) 427.

51 Hasani (2003) 85-97.

52 Hespanha (1996). 
from Roman law to achieve many different aims; nevertheless, this is a well studied subject that leads us astray from our reflection on the origins of the uti possidetis doctrine. ${ }^{53}$

At all events, one thing is undisputable, namely, that the sentence passed by the Court would have satisfied the Chilean diplomat and jurist Alejandro Álvarez, whose theses on the existence of an international law specific to America influenced to a large extent the "international lawyers" debate during the first half of the $20^{\text {th }}$ century. ${ }^{54}$ According to Álvarez, the use of the Monroe doctrine in Latin America, implying the refusal to consider the so-called 'western hemisphere' as terra nullius, ${ }^{55}$ conditioned the emergence of an American international law. «The entry of Latin America into the community of nations is one of the most important facts inthe history of civilization", stated Álvarez in the opening paragraph of his famous article, ${ }^{\mathbf{5 6}}$ in which he highlighted the role played by the uti possidetis doctrine in defending Latin American territorial integrity against foreign interference. ${ }^{57}$ Nevertheless, Álvarez underscored the differences that existed between old Europe and the new America; in his own words: «Europe is formed of men of single race, the White; while Latin America is composed of a native population, negroes imported from Africa, and the Creoles». Therefore, Álvarez did not overlook to identify the ruling group in that new America: according to him, the Creole element was «the only thinking part of the population». ${ }^{58}$

Many Latin American internationalists accepted the Chilean jurist's thesis, while others were critical of it. ${ }^{59}$ This discussion lost interest for inter-

53 KoskenNiemi (1997) 215-263; Lesaffer (2005) 25-58.

54 Becker Lorca (2006a) 879-930.

55 President Monroe deliberately used the term "this hemisphere" in his message on 2 December 1823, to indicate that the political system in the western hemisphere, understood as a regime of freedom, was frontally opposed to the European monarchic political system. On this subject, vid. Sснмітт (1979) $364 \mathrm{ff}$.

56 Álvarez (1909) 269.

57 Castellino (2008).

58 Álvarez (1909) 271-272.

59 Among the critics was the voice of the Brazilian Manoel Álvaro de Souza Sá Viana, who denied the existence of any specificity, alleging that the Latin American version was just one more among others in international law. Souza Sá Vianna (1912). 
nationalists from the $1950 \mathrm{~s},{ }^{\mathbf{6 0}}$ and today it has become both a historical source and object for recent internationalist historiography which, firmly anchored to the present, ${ }^{61}$ has revisited the origins of uti possidetis analysing and relating them with the role of the latter in the international order. ${ }^{62}$ It must be stressed that most of such studies can be classed under what some have called the «historiographical turn in international law», ${ }^{63}$ understood as the insertion of the historical and theoretical dimensions of international law into a single field of research. ${ }^{64}$ Indeed, in the last three decades an extremely powerful historiography has emerged and become consolidated holding a highly critical view of the doctrines of international law, ${ }^{65}$ by virtue of which it has turned the historical tradition of the discipline into a research object. ${ }^{66}$ This understanding has thrown onto the ropes the strategy that, based on the reiteration of a collection of set phrases, has distorted the chronology of the stages in the formation of this discipline, ${ }^{67}$ and contributed to concealing the persistence of a set of ideas that are structural to international law. ${ }^{68}$ In a nutshell, and as I believe P. Costa would have put it, thanks to this "historiographical turn in international law" history is being written today about the tradition rather than in the tradition. ${ }^{69}$

In more concrete terms, the focus of this Latin American historiography has been on analysing the works of its most distinguished jurists, ${ }^{\mathbf{7 0}}$ from

60 Some authors advocate forgetting the classic Latin American pathologies when making proposals for the future; in this sense, vid. Esquirol (2011).

61 Becker Lorca (2006b). For its references to American authors, vid. also De la Rasilla Moral (2013).

62 Castellino/Allen (2003).

63 Bandeira Galindo (2005).

64 Bandeira Galindo (2012) 86.

65 Koskenniemi (2004a); Carty (1991); Beneyto/Kennedy (2012).

66 Kennedy (1988). One of the most significant items of common knowledge is locating the origins of the discipline in the works of its alleged founding fathers such as Vitoria or Grocio (Nuzzo/Vec [2012]), which implies the denial of the internationalist doctrine's essentially nineteenth-century roots (Koskenniemi [2004b]; Anghie [2005]).

67 Kennedy (1999) 100.

68 ANGHIE (2006). In an earlier work, this author affirmed: «The colonial encounter, far from being peripheral to the making of international law, has been central to the formation of the discipline. By this I mean not merely the specific doctrines of the discipline, but its informing philosophy: positivism» (ANGHIE [1999] 70).

69 Costa (1989).

70 Becker Lorca (2006a). 
which can be inferred the keys to what some authors call "Creole international law".71 As a general rule, the use of this adjective is understood in the terms used by Brading, ${ }^{72}$ to which we may add that said law not only reproduced and made use of foreign constructs, but it also contributed to the globalization of international law. ${ }^{73}$ At all events, and although this problem is overcast by the long shadow of the New World dispute, ${ }^{74}$ it is not exclusive to international law. ${ }^{75}$ What I would like to emphasize is that some authors have adopted almost in full the historical narrative drafted by the first Latin American internationalists regarding the origins of the $u t i$ possidetis principle: such is the case, for example, of the excellent work by Castellino and Allen, who nevertheless show little interest in learning more of Latin American history than that provided by Álvarez for the analysis of what they have termed «Spanish America and the Treatment of Territory in International Law». ${ }^{76}$

The consequences of using the works of early Latin American international lawyers as a source are not exactly of minor importance. To begin with, this practice implies either unwitting ignorance or deliberate dismissal of a juridical historiography whose efforts for the last few decades have been dedicated largely to contextualizing pre-modern juridical culture. To do so, this historiography has had to negotiate many obstacles, in particular those originating from its own disciplinary tradition, in that the latter set the teleological component as its primary objective. We should keep in mind that this hasty diagnosis is valid for both Europe and America, from which we can deduce not only new angles to regard the traditional History of

71 Obregón (2006). I have not gained access to a well cited work by this author: Obregón (2002).

72 Brading (1991).

73 Becker Lorca (2010). Two critiques of the theses maintained in this article are found in: Özsu (2010) and Gozzi (2010).

74 Gerbi (1960).

75 In a recent publication, José Antonio Aguilar reflects once again on the feebleness of Spanish American thinking, highlighting the exceptions to the logic of acritical intellectual appropriation that repeatedly appears in the Spanish American juridical-political culture. «Los hispanoamericanos repetimos fórmulas hechas y compramos teorías que rara vez examinamos a fondo. Nuestro hábito es el consumo, no la producción intelectual», states the autor in his conclusions. Aguilar Rivera (2012) 322.

76 Castellino/Allen (2003) 57-78. 
Indiano Law, but also new conclusions that affect its structural elements. ${ }^{77}$ In short, I fear that some of the authors fuelling the "historiographical turn in international law" are not fully aware of the "historiographical turn in legal history". Thereby not only will errors and confusion accumulate curtailing dialogue of any sort, but in some instances they will act against the main theses put forward by the new breed of historians and internationalists.

The latter is the case of some of the writings of Liliana Obregón, ${ }^{78}$ who has coined the successful expression 'Creole international law' identifying the specific traits of Latin American legal consciousness. ${ }^{79}$ Obregón states that nineteenth-century lawyers, from among whom the future internationalists were to emerge, thought of European law as something susceptible of being appropriated owing to the shared legal tradition based not only on the validity of Castilian sources in the Indies, but also on the manner in which they were enforced throughout colonial times. According to Obregón, the Indies' special conditions - distance, diversity, etc. -, called for a flexible approach, and therefore casuistry and judicial arbitration were dominant in the administration of Indiano legal order. ${ }^{80}$ However, Obregón's understanding of the regulations of the Catholic Monarchy is very traditional, leading her to take as Creole some structural elements of the legal order in pre-revolutionary Europe. ${ }^{\mathbf{8 1}}$ If legal historiography has proved anything for some time now, it is that casuistry and arbitration were consubstantial with the European culture of ius commune, ${ }^{\mathbf{8 2}}$ whose transferral to the Indies meant no essential change whatsoever. ${ }^{83}$ All Hispanic territories were governed in the same heterogeneous fashion, ${ }^{\mathbf{8 4}}$ which basically meant according to their own rights, or in the case of the Indies, according to their customs, ${ }^{\mathbf{8 5}}$

77 Tau Anzontegui (1997).

78 Obregón (2006) and (2009).

79 Kennedy (1980).

80 L. Obregón maintains these statements referring to a work by Cutter, in which this author uses the expression derecho vulgar to describe the process adapting Castilian law to American needs.

81 Garriga (2004a).

82 Meccarelli (1998); Tau Anzoátegui (1992). A good summary of juridical culture during the Catholic Monarchy is found in AgüEro (2006).

83 Tau Anzoátegui (2002); Nuzzo (2008). Recent studies show that the transplant was successful, even on the periphery of the periphery: AgüEro (2008); Zamora (2010).

84 Garriga (2004b).

85 Barrientos Grandón (2000). 
the whole being administered by lawyers educated in a common creed on both sides of the Atlantic Ocean. ${ }^{\mathbf{8 6}}$ In this scenario, the elements from which Obregón infers an alleged American juridical identity and extracts there from the term criollo (Creole) to label Latin American international law were in no way specific to the legal order of the Reynos de las Indias (Kingdoms of the Indies). ${ }^{87}$

Any research on uti possidetis with a historical bent comes up against a mass of information distributed across myriad sources and an excessively heterogeneous bibliography. Of course, these features are common to any historical research, but the Latin American historiography generated through uti possidetis is characterized by being constitutive of the territory whose history it is supposed to be drafting. Furthermore, this historiography has another not unworthy quality, namely, its foundational nature with respect to Latin American national historiographies. There is no need to consult recent historical research to ground the foregoing statement, since the internationalist Carlos Calvo highlighted this fact as long ago as the 1860s; in his own words:

«South America in its colonial state had nothing; it found little on gaining life of its own, and has not progressed far on that road; it needs, therefore, to create everything on its way. It often happens, when the time comes to discuss limits for their final settlement, that they need to consult the archives and libraries in the motherland, and more often than not those of Paris and London». ${ }^{\mathbf{8}}$

The first works written on colonial sources in the Spanish archives throughout the $19^{\text {th }}$ century, ${ }^{89}$ and the creation and organization of the archives on the new American states, ${ }^{\mathbf{9 0}}$ were both in response to the "boundaries question" that arose after Latin American independence. However, the search for titles to prove territorial rights acquired according to the rule of uti possidetis to a large extent determined Latin American historiography beyond its original function, ${ }^{91}$ insofar as the application of a series of territorial truths became a constant that has continued to the present day. ${ }^{92}$

86 Barrientos Grandón (2004) and (2001).

87 Garriga (2004a).

88 Calvo (1862) t. I, XLV.

89 VÉLEZ (2008) 247.

90 Podgorny (2011).

91 Molina (1955).

92 Peralta (1886). 
Having arrived at this point, let us recapitulate. On the one hand, we have a traditional doctrine that prides itself in the American contributions to international law among which one the most outstanding is the uti possidetis rule. On the other hand, we have another that, despite being critical of doctrinal traditions, nevertheless tends to reproduce the historic narrative used by the first Latin American international lawyers. We shall not insist for the moment on the first, but rather focus on the consequences of the failure in the case of the second to include the more significant contributions made by American historiography in the last few decades.

\subsection{Colonies, Independence and Historiography}

Álvarez did not dwell on the role of the uti possidetis doctrine in the formation of the new American states; as might be expected, the Chilean jurist had no wish to emphasize the underlying weakness of the new American states. From today's perspective, however, an almost insurmountable barrier separated the function attributable to uti possidetis regarding the relationship between America and the European powers, eager for expansion following the collapse of the Catholic Monarchy, from that which is presumed to have regulated the distribution of territory among the American élites, equally eager to become consolidated and even for enlargement, after having gained their independence.

On identifying uti possidetis mainly with the refusal to consider Latin America as terra nullius, Álvarez not only concealed the consequences of its implementation from the indigenous population, but also tiptoed over the difficulties that arose during the administration of the process by the Creoles themselves. ${ }^{93}$ Of the consequences mentioned above, one in particular stands out, namely, that the revocation of treaties signed with the Indian nations enlarged the territory of the new, civilized, Latin American states, ${ }^{\mathbf{9 4}}$ independently of the conversion of Araucans, Pampas or Apaches into citizens of the new Republics being a fiction fraught with disadvantages for the latter. ${ }^{95}$ Among the difficulties mentioned, one also stands out significantly: the quarrels among Creole élites discredited any possible respect for the

93 Ireland (1940).

94 LeVAGgi (2002).

95 Clavero (2005); Weber (2007). 
former colonial divisions when marking out the territorial boundaries for the new republics. To sum up, while the uti possidetis doctrine achieved grosso modo the first objective by establishing that emancipated America was not terra nullius, ${ }^{\mathbf{9 6}}$ the statement that the distribution criterion on which the Monarchy's famous boundary demarcations were the basis for establishing boundaries is largely a retrospective fiction, refractory to becoming an object of history.

The supposed continuity between the territory of the Monarchy and those corresponding to the new republics, often equated with state succession, met with an objective pitfall at the time, regardless of this being used as a weapon in the new political leaderships' territorial struggles. Expressed in interrogative terms: what were the Monarchy's territorial demarcations? ${ }^{97}$ Or, alternatively, were the Viceroyalties, Audiencias, Intendencies or, conversely, other minor units, expected to determine the spatial limits of the new states? Internationalist historiography usually hastens over such questions although they are essential to understanding in all its density the rule in hand. On many occasions, scholars have suggested without actually stating it as a fact that whereas uti possidetis has been acknowledged both in constitutional charters and in American international treaties, this rule was largely a right pre-constituted in colonial times that metamorphosed after independence into the generic Creole gentlemen's agreement referred to by Álvarez when identifying the spirit of uti possidetis. ${ }^{\mathbf{9 8}}$ Nevertheless, the priority given to its 'interior' or constitutional use over 'exterior' or international use, has been pointed out by the Court itself in negative terms: «It should be recalled that when the principle of the uti possidetis juris is involved, the jus referred to is not international law but the constitutional or administrative law of the pre-

96 «Hence there is no territory in Spanish-America that has the status of res nullius open to an acquisition of title by occupation» in Dispute between Argentina and Chile (1977).

97 SCHRÖter (2001).

98 Not only Álvarez; cfr. the following text by a former President of the II Spanish Republic: «Los pueblos hispanoamericanos aparecen con la filiación más legítima, consciente, jurídica y culta que pueda encontrarse. Los más de los otros Estados, quizá todos han surgido de la fusión violenta, sanguinaria y guerra, como esos hijos, más del rencor que el deseo, engendrados entre los incendios y saqueos de las ciudades asaltadas, Solo esta filiación hispanoamericana aparece deliberadamente bendecida por la fe y sancionada por la ley, en una obra reflexiva de fecundidad civilizadora». Alcalá Zamora (1944) 153. 
independence sovereign». ${ }^{99}$ Indeed, «[...] it should not be overlooked that Spanish colonial divisions in Spanish America did not individually have any 'original' or 'historic' titles, as those concepts are understood in international law. The original title belonged exclusively to the Spanish crown, not the internal administrative subdivisions established by it; and it was equally the Spanish Crown which had sovereignty of the colonial territories». ${ }^{\mathbf{1 0 0}}$

Independence granted the new nations their title to territory; they, in turn, looked to the Monarchy's legacy for projecting this onto physical space in order to create borders where previously there had been limits or boundaries. ${ }^{101}$ This strategy involved recording the landmarks of a political-constitutional history, which have become common knowledge even for the most critical internationalists. The following tale may serve as an example to illustrate this: At the behest of Libertador Simón Bolívar, the Venezuelan Congress - which had elected him as president of the republic - enacted in Angostura the Basic Law of 1819 that included the uti possidetis doctrine in its articles: «The Republics of Venezuela and New Granada are from this day united in a single Republic, under the glorious name of Republic of Colombia»; «Its territory shall comprise that of the ancient Captaincy General of Venezuela and the Viceroyalty of the New Kingdom of Granada, encompassing an extension of 115 thousand square leagues, the exact boundaries of which shall be agreed under better circumstances». Other texts show that this idea was not exclusive to a portion of the Monarchy's former territories: for example, whereas the Mexican Constitution of 1824 asserted that «The Mexican State is composed of the provinces contained in the Viceroyalty formerly called New Spain; of the province formerly called Captaincy General of Yucatán, and of the Commandancies General of the eastern and western interior provinces», the Constitution of the United Provinces of Central America, promulgated the same year, stated «The territory of the Republic is the same that belonged to the ancient Kingdom of Guatemala, with the exception, for the present, of the Province of Chiapas». The Constitutions gave way to regional treaties: as Álvarez expressed it, «Failing the confederation of the Latin states, the problem of the marking of the boun-

99 Land, Island and Maritime Dispute (1992) 559.

100 Land, Island and Maritime Dispute (1992) 565.

101 Marchetti (2001). 
daries which the pacts of confederation strove to solve, of necessity came day by day more prominently to the fore. The constitutions of some of the countries fixed their lines on the basis of the uti possidetis of 1810, which was, moreover, recognized in fact by all the states, and proclaimed in the Congress at Lima in 1848». ${ }^{\mathbf{1 0 2}}$ The cycle had ended.

Although acceptable in part, narratives of this type bar the possibility of writing up a history of the territory which departs from the double constitutive valence of historiography placed at the service of uti possidetis. To this end, it is indispensable to be familiar with the turn taken by American historiography in recent decades, which has served to cast off the traditional stays that corseted the major national narratives of the $19^{\text {th }}$ century. ${ }^{103}$ As it is impossible to give a full balance of the new order of things, I shall be content with underscoring the most important aspects which are those generically or directly related to uti possidetis in wholly different terms to those employed by such internationalists as Álvarez in his time.

Firstly, the thesis of Halperin Donghi, ${ }^{\mathbf{1 0 4}}$ according to which Americans were obliged to opt for an independence that they did not want, ${ }^{\mathbf{1 0 5}}$ has gained an increasing number of followers. ${ }^{\mathbf{1 0 6}}$ Secondly, the alleged republican sentiment, ${ }^{\mathbf{1 0 7}}$ that was supposed to identify Latin American states with the United States of America, has been questioned again, since not only did many American Constitutions opt for Monarchy, but also many elements of a monarchic nature were later maintained under republican trappings. ${ }^{108}$ Thirdly, many scholars have been reduced the influence of natural law thought on Latin American institutional constructions, ${ }^{\mathbf{1 0 9}}$ as it has been demonstrated that preserving a juridical-political imaginary, both Catholic and anti-individualistic, ${ }^{\mathbf{1 1 0}}$ translated into the persistence of former institutional measures well into the $19^{\text {th }}$ century. ${ }^{111}$ And, to finish, the massive

102 Álvarez (1909) 290.

103 Palacios (2009).

104 Halperin Donghi (1985).

105 Meglio (2008).

106 Rodríguez (2008); Portillo (2006).

107 Aguilar Rivera/Rojas (2002).

108 O'Gorman (1986); Landavazo (2001).

109 Armitage (2007).

110 LeMpÉrière (2004) (2005b).

111 Lorente/ Portillo (2012). 
municipal explosion, that practically disintegrated all of Spanish America after $1808,{ }^{112}$ is today the starting point for most researches on the period, ${ }^{113}$ some of which have also noted the international nature of the relations among American municipalities or provinces. ${ }^{114}$

All the above goes a long way toward explaining the obsession experienced by the earliest drafters of Constitutions to include in their texts a description of the territory encompassed by the new republics. In this sense, one of the most paradigmatic examples were offered by the neo-Granadine Constitutions, that were drafted, approved and even amended throughout the period known as la patria boba (the foolish fatherland). ${ }^{\mathbf{1 1 5}}$ Thus, for example, Article 17 of the Constitution of Popayán, enacted by the Most Serene Electoral and Constituent College in 1814, stated: «The territory of the province $[\ldots]$ comprises the area between the eastern and western Andes, as well as the extension between the Pacific Ocean to the west as far as the barbarian Andaquíes nations to the east, the municipalities of Popayán, Cali, Buga, Caloto, Cartago, Anserma, Toro, Almaguer, Pasto, Barbacoas and Inscuandé, and the possessions of Raposo and Micayun». ${ }^{116}$ As you will have observed, the constituents of Popayán made no mention whatsoever of maintaining the boundaries of the ancient province; to the contrary, in addition to acknowledging the territoriality of the 'barbarian nations', they formally recorded the political bodies that made up Popayán. Nevertheless, other Constitutions adopted more geographical criteria, for instance, that of the State of Cartagena in 1812, but on closer examination, this text is found to preserve jurisdictional conceptions of space. Indeed, in the description of the State's territorial limits contained in Article 6, the Constitution of Cartagena stated that the ownership of the islands in the Magdalena River must be discussed by the General Congress of the Kingdom, considering that they had been «awarded exclusively to one of the adjacent provinces by virtue of

112 Annino (1995).

113 Verdò (2006a) and (2006b). The cases of Venezuela and Colombie in Hébrard (1997) and Calderón (2010). And, lastly, the case of Quito, in Morelli (2001) (there is a Spanish edition of the latter work translated by Hermosa Andujar [2005]).

114 Gutiérrez Ardila (2010).

115 Castro Leiva (1991).

116 Restrepo Piedrahita (1996). 
laws enacted in ignorance of the facts, without a hearing of the parties, and perhaps against the mandate of nature». ${ }^{\mathbf{1 1 7}}$

By 1810 , the date of the uti possidetis, ${ }^{118}$ territories had to be formed according to the old logic of aggregation of political bodies. Cities, provinces and, finally, states, held or claimed their ownership. ${ }^{119}$ Few examples are more illustrative of this process of dismemberment and integration than that of Quito, later to become Ecuador. The Constitution of Quito (1812) highlighted this in its first Article: «The eight free provinces represented in this Congress, and evermore inextricably united from this moment, shall permanently form the State of Quito as integral parts thereof $[\ldots] \gg .{ }^{\mathbf{1 2 0}}$ Note that in this Article no mention is made either of former boundaries, to which we must add that it was not until the Constitution of 1852 that any comparison is made of the new Ecuadorian and the old colonial territories, that is to say, to find evidence of the constitutionalization of the uti possidetis doctrine: «The territory of the Republic comprises the provinces that made up the ancient Presidency of Quito and the Galapagos Archipelago. Its boundaries shall be definitively established through treaties signed with the neighbouring States» (Article 3). ${ }^{\mathbf{1 2 1}}$ This formula is found repeated, grosso modo, in subsequent Constitutions until that of $1998,{ }^{122}$ and only the first Ecuadorian Constitution in force offers a different formula. ${ }^{\mathbf{1 2 3}}$ Considering the huge

117 Constitución del Estado de Cartagena (1813).

118 See supra.

119 Chiaramonte (1997).

120 http://bib.cervantesvirtual.com/servlet/SirveObras/01372764257917832311802/index.htm.

121 http://bib.cervantesvirtual.com/servlet/SirveObras/02438343103132163754491/p0000001. htm\#I_2_.

122 Art. 2: «The territory of Ecuador is unalienable and irreducible. It includes that of the Real Audiencia of Quito with the modifications made by the treaties in force, the adjacent islands, the archipelago of the Galapagos Islands, the territorial sea, and the ground beneath and the space over said land» (available at: http://bib.cervantesvirtual.com/servlet/ SirveObras/01371296122384892980035/p0000001.htm\#I_2_).

123 Article 2 of the Constitution of 1998. The current Constitución Politica de Ecuador (2008) has discontinued this tradition in Article 4: «The territory of Ecuador constitutes a single geographical and historical whole, with natural, social, and cultural dimensions, which has been passed on to us by our ancestors and ancestral peoples. This territory includes the mainland and maritime space, adjacent islands, the territorial sea, the archipelago of the Galápagos Islands, the land, the undersea continental shelf, the ground under the land and the space over our mainland, island, and maritime territory. Its boundaries are those 
territorial losses suffered by Ecuador until recent times, it is surprising to find such insistence on mentioning over and again the territory of the Presidency and Audiencia of Quito, ${ }^{124}$ to which we should add that, on occasion, said territory has been identified with that of the ancient Quichua kingdom, ${ }^{\mathbf{1 2 5}}$ whose existence owes so much to the Jesuit historiography of the seventeenth century. ${ }^{\mathbf{1 2 6}}$ The Monarchy's old demarcations did not play a prominent role immediately after emancipation. Only after the revolutionary storm had abated, did the new, civilized republics establish a definitive land-sharing criterion that depended on a colonial history of the territory to

determined by treaties currently in force. The territory of Ecuador is unalienable, irreducible and inviolable. No one shall jeopardize its territorial unity or foment secession. The capital of Ecuador is Quito. The Ecuadorian State shall exercise its rights over those segments pertaining to the geosynchronous orbit, the maritime space and the Antarctic».

124 Sánchez Bella (1980). An example of the Audiencia's territorial delimitation can be followed in the royal cedula dictated by Philip II in the city of Guadalajara, on 23. August 1563: «In the city of San Francisco del Quito, in Peru, may another Audiencia y Chancilleria Real [...] be established, whose district shall comprise the Province of Quito, and along the coast toward the city of kings (Lima) as far as the port of Payta exclusive, and its inland territory as far as Piura, Caxamarca, Chachapoyas, Moyobamba and Motilones exclusive, comprising toward said parts the towns of Jaén, Valladolid, Loja, Zamora, Cuenca, la Zarça and Guayaquil, with all other towns lying within their districts or that later might be settled: and in the direction of the towns of Canela and Quixos, let these be included and any others that might be discovered: and following the coast toward Panamá, to the port of Buenaventura inclusive: and inland toward Pasto, Popayán, Cali, Buga, Chapanchica and Guarchicona: because the remaining places under the Gobernación de Popayán belong to the Audiencia of the New Kingdom of Granada, with which, and with the mainland, the North boundary is drawn; Reyes to the South, the Southern Sea to the West, and to the East, provinces as yet unpacified or undiscovered». Included in Law X (Audiencia y Chancillería Real de San Francisco del Quito) under Title XV (De las Audiencias y Chancillerías Reales de las Indias) of Book II of the Laws of the Indies, 1680.

125 Constitution of 1830, Article 6: «The territory of the State included the three departments of Ecuador on the edge of the ancient Kingdom of Quito». Constitution of 1845, Article 1: «The territory of the Republic of Ecuador, composed of the districts of Quito, Guayas and El Azuay, on an equal representation basis, comprises all the provinces of the ancient Kingdom and Presidency of Quito, including the archipelago of Galápagos, whose main island is known as Floriana. The boundaries of this Republic shall be definitively established by public treaties with our neighbouring Nations».

126 Velasco (1841-1844) (Juan de Velasco completed this work in 1789; I have used the edition published in Quito, Casa de la Cultura Ecuatoriana, 1977, available at: http:// www.bibliotecayacucho.gob.ve/fba/index.php?id=97\&backPID=103\&begin_at=72\&tt_ products $=82$ ). 
become effective. Said criterion, uti possidetis, materialized in the international (regional) order and in the national (state) order almost simultaneously, more or less concurrent with the Congress held at Lima in 1847-1848, at which it was affirmed as an American principle of international law. ${ }^{\mathbf{1 2 7}}$ Hence, in Spanish America the principle did not serve «to prevent the independence and stability of new States», but was used to create them. However, it is commonly said that uti possidetis was a rule made for the preservation of «the territorial status quo which existed when they obtained Independence», without remarking that, with this stroke of timelessness, the Court contributed in no small measure to strengthening the old Latin American tradition that envisioned not only nations, but states too, well before the collapse of the Catholic Monarchy. Thus, for example, Bernardino Bravo Lira, law historian and recently awarded the national prize for History, insisted on this only a short time ago: «Unlike events in nearly the whole of Spanish America, in Chile we find what in Spenglerian terms I would call a State 'in form'. In this respect, independence merely represented a parenthesis that was closed with the restoration of the same State under a new modality». ${ }^{128}$

I shall not dwell on the difficulties surrounding the naturalization of key terms in our language, such as State or Nation, and their projection in Latin America, ${ }^{129}$ but I will attempt to identify some of their consequences in the field determined by uti possidetis. Today, discussions are ongoing on the contradiction involved in using two principles that are recognized in international law as being contradictory: in absolute terms, the right to selfdetermination and the principle of uti possidetis are irreconcilable, as demonstrated in some flagrant cases such as Western Sahara. Transcending these tensions, however, it should be noted that one thing is to claim the succession of a colonial, but statalized, territory, and quite another to expect to do the same in another which, for merely chronological reasons, had never experienced the consequences of a statalized concept of political power. ${ }^{\mathbf{1 3 0}}$

Undoubtedly, this last scenario is the Latin American case; consequently, the territorial history of the Monarchy governed according to the uti possidetis

127 Kohen (2009).

128 Bravo Lira (1996) 13.

129 Chiaramonte (2004).

130 LEMPÉRIÈre (2005a). 
principle has a conventional value equivalent to a sort of procedural truth that may or may not be accepted by the parties. ${ }^{\mathbf{1 3 1}}$ However, establishing territorial truths not only requires agreement over the narrative itself but also in the way it is composed, and this, in turn, draws veritable borders between historiographies. In still clearer terms: everything seems to indicate that the territorial truth that was and is used in issues over boundaries has proved incapable of assimilating any degree of historiographical innovation, especially any changes causing the breakdown of nationalist and / or statalist views of a retrospective nature.

\section{Fictions of the uti possidetis doctrine}

In analysing the uti possidetis doctrine, internationalists usually differentiate between uti possidetis iuris and uti possidetis de facto, and draw comparisons between the dates of 1810 and 1821 . Without wishing to address this complex matter in depth, we can safely say that whereas the first question is projected on the territory, the second involves time, and, finally, that both refer to Latin American history. This starting point overlooks a well-known fact, namely that the acceptance of the uti possidetis principle carries implicit the annulment of indigenous time and space. ${ }^{\mathbf{1 3 2}}$ This is not tantamount to affirming that spatial and temporal perceptions were not made use of by the conquistadors in their time, ${ }^{\mathbf{1 3 3}}$ who successfully colonized the indigenous imaginary, ${ }^{\mathbf{1 3 4}}$ nor that they disappeared completely, because they were sometimes brought back and used by the indigenous population, ${ }^{\mathbf{1 3 5}}$ but today they have no place in international law. The International Court of Justice has been very clear on this point: «It was the administrative boundaries between Spanish colonial administrative units, not the boundaries between Indian settlements as such, that were transformed into international boundaries in $1821 »{ }^{\mathbf{1 3 6}}$ It is no coincidence, therefore, that so many internation-

131 Semana (2012).

132 Florescano (1994b).

133 Lockart (1999) especially chapter I, titled "El Altépelt", 27-88.

134 GRUZINSKI (1991).

135 Carmagnan (1988).

136 Caso relativo a la controversia sobre fronteras terrestres, insulares y marítimas (El Salvador contra Honduras: Intervención de Nicaragua), awarded at the International Court of Justice on 11 September 1992 (available at: http://www.icj-cij.org/homepage/sp/files/ sum_1992-1996.pdf). 
alists should be critical of the consequences of implementing the principle from the standpoint of defending indigenous rights.

However, the 'fictions' referred to in the heading are but indirectly related with plunder, present and past. In my opinion, the uti possidetis is based on a 'mirror game' that reaches beyond the task of fiction-writing consubstantial to juridical science. The uti possidetis charges the history of Latin American territory with the responsibility for establishing borders, while at the same time building said history on the basis of a series of temporal and spatial conventions that are questionable, at least as far as historiography is concerned. On the one hand, the uti possidetis owes its existence to two key dates, 1493 and 1810, that originate, respectively, from a Catholic perception of time and from Latin American national mythologies. On the other hand, these same dates refer to property rights conventions that do little to address the effective control of space as an objective phenomenon. All in all, however, I believe it is necessary to clarify that the above statements do not in any way detract from the pacifying function that the use of the uti possidetis may have had - or still have - in controversies over borders; the aim here is to establish a generic assessment of its historiographical premises/consequences. $^{137}$

\subsection{Dates and Titles. From Bulas to Independencias, revisiting} the Chinese histories

Among internationalists, the uti possidetis is not often associated with the Alexandrine Bulls because these papal titles seem to us today rather distasteful in terms of international law. Generally speaking, it is the emancipation of America, understood as the first decolonization movement that appears when at all - in the writings of those international lawyers. ${ }^{\mathbf{1 3 8}}$ The terms 'scarce' and 'non-existent' are of course similar, but have different meanings

137 Hensel/Allison (2004).

138 Some authors claim that the earliest origins of the principle should not be sought in in the American emancipation but in the failed Treaty of Madrid which, signed on 13 January 1750 , aimed to redefine the boundaries of the Iberian Monarchies referring exclusively to the uti possidetis, or the treaty signed on 1 October 1777 in San Ildefonso which, in general lines, rehabilitated the former. At all events, these two instruments responded to an issue that began with the Treaty of Tordesillas of 1494 and, therefore, the papal bulls. Riвот (1995). 
as highlighted by those who hold that the territorial 'rights' of Latin American states, Chile in this case, «were granted by the titles (papal bulls included) in possession of the Spanish crown over the continent $[\ldots]$ of Antarctica». ${ }^{139}$ These individual voices were joined by others of an official nature, insisting on the currency of the pontifical provisions: «The Falkland Islands formed part of the area under Spanish jurisdiction [...]. The papal bulls and the Treaty of Tordesillas of 1494 were the first instruments to reflect Spanish titles in accordance with the international law of the period».140

Whether they were used or not, the bulls could be exhibited as the first historical title held by any state over a given territory. Traditionalists and critics alike agree on this point, although they naturally differ in their valuation. Thus, whereas the first defend the current effectiveness of the pontifical titles, justifying them on the well-tried grounds of affirming their applicability to an indeterminate "international law of the period" which they do not generally take the trouble to analyse, the second make use of them to single out and denounce that great original sin of international law: in the words of Anghie, «The colonial encounter, far from being peripheral to the making of international law, has been central to the formation of the discipline». ${ }^{\mathbf{1 4 1}}$ All in all, the rivers of ink that have been spilt on bulls and treaties advise against returning to the question, despite which it is well to recall some aspects closely related not only to the uti possidetis in particular but also to the political-legal use of the history of the territory in general.

It is common knowledge that the great discoveries of the $15^{\text {th }}$ and $16^{\text {th }}$ centuries, or perhaps, 'the discovery of humanity', ${ }^{\mathbf{1 4 2}}$ paved the way for a new "global view of space that required a new global spatial order». ${ }^{143}$ Its foundations were rooted in the bulls, which constituted the main evidence that it was possible to «confinare non solo la terra, ma anche la vastità del mare». ${ }^{\mathbf{1 4 4}}$ However, the papal awards of non-Christian territories - the bulls

139 San Miguel Casisa (2013).

140 Embajada de la República Argentina en la República Federal de Alemania: http://ealem.mrecic.gov.ar/node/3686.

141 Anghie (1999) 70. On this same question, Anghie (1996); Koskenniemi (2009).

142 Abulafia (2009).

143 SСHмiтt (1979) 74.

144 Marchetti (2001) 16. 
- that were implemented by the Christian sovereigns assuming those lands and waters - by means of treaties -, had their origins in «[...] the spatial order of the medieval Respublica Christiana». ${ }^{\mathbf{1 4 5}}$ It is a fact that the bulls did not refer to a concrete space, as this was still to be discovered, but were missionary requests that brought with them the juridical culture of the ius commune.

In his excellent work, Marchetti has described spatial perception within that culture, stressing its imperial dimension: «Si è spesso incrociato con l'analisi di una forma di organizzazione politica, quella imperiale; non foss'altro per il fatto che due imperi (L'Impero cinese e l'Impero romano) hanno lasciato una memoria talmente profonda delle loro fortificazioni di frontiera da non permettere di trascurare l'osservazione del rapporto esistente tra modelli organizzativi, storicamente dati, di tipo imperiale e i loro confini». ${ }^{146}$ Once again, however, the bulls highlighted the fact that the successors to the Roman Empire believed they could wipe the Chinese from the face of the Earth. Indeed, although initially no one thought of the consequences that the line drawn by the Pope may have in the other hemisphere, the notion of an 'antimeridian' immediately appeared which obviously affected the territory of the Celestial Empire. ${ }^{147}$ Prompted by an evangelizing spirit, the mapping of the antimeridian was determined by the distribution of rights and obligations among the Christian princes; hence it is no coincidence that the Emperor Charles should have addressed his explorers in these terms: «refrain from discoveries or any actions within the demarcation or limits of the Most Serene King of Portugal, my beloved uncle and brother, nor against his interests, save within the limits of our own demarcation». ${ }^{\mathbf{1 4 8}}$

«Thanks to the Papal Bull, the Crown of Castile could have established its sovereign rights over China and the Malay Peninsula», writes Padgen in an excellent book. ${ }^{149}$ However, this author - or, perhaps, his translator - uses the wrong tense, since by the end of the $15^{\text {th }}$ century the unevangelized spaces of the globe were conceived as res nullius. Explorers and conquista-

145 Sснмiтt (1979) 82.

146 Marchetti (2001) 63.

147 Pérez Bustamante (1922).

148 Cit. in Exposición Pacífico (2013) 129.

149 Padgen (1991) 31. 
dors, jurists and clerics insisted time and time again on the alleged rights of their monarchs to conquer the world. ${ }^{\mathbf{1 5 0}}$ «I have faith in God that from this small beginning He will enlarge and increase the kingdoms and seigniories of your Majesty, and we shall be able to carry the true knowledge of the holy Catholic faith to so many barbarous and blinded men who are found in these regions, including the vast kingdom of China and many others. Heaven has this good fortune in store for your Majesty; so that it may be fulfilled during these propitious times of your Majesty», stated the second Governor of the Philippines in a letter to Philip II in $1574 .{ }^{151}$ Plans for the so-called "China enterprise" ${ }^{152}$ were followed by others of a similar nature, ${ }^{153}$ calling for the conquest of Siam, Champa, Cambodia and Cochin-China. ${ }^{154} \mathrm{Cam}$ panella's delusions of grandeur were not exclusive to this famous cleric, ${ }^{155}$ despite which we know that success did not accompany such mad enterprises, which among other things threatened to substitute the measurement of time in the Celestial Empire with the Catholic measure, through subjugating its subjects to the designs of the Maker, in whose spirit, as Saint Augustine said, tempora metior. ${ }^{156}$ And so, the classical Chinese representa-

150 Padgen (1997).

151 AGI (Archivo General de Indias), Patronato, 24.

152 Ollé (2002).

153 Copia de carta de fray Martín de Rada al virrey de Nueva España, informando del estado de las Filipinas: descripción de las islas y lugares como Luzón, Borneo, Panay y Masbate, de sus riquezas, pobladores, comercio, esclavitud entre ellos, y abundancia de oro. Opina que es mejor conquistar esas tierras con pobladores que con soldados, pues los naturales no tienen rey, señor, ni leyes, y es fácil dominarlos. Destrozos que han hecho los españoles, incontrolados por falta de orden y autoridad del gobierno, lo que también ocasiona que sean incapaces de mantenerse en una tierra rica y fértil. Si se pretende pasar a China, de la que se tienen buenas noticias y perspectivas, hay que asentar primero la conquista de las Filipinas. AGI, Filipinas, 79, 1, 1.

154 Carta de Fernando de los Ríos Coronel dando cuenta del astrolabio que había inventado; de lo importante que sería la conquista de Siam, Camboya, Champa y Cochinchina; de la conveniencia de ocupar isla Hermosa y descripción detallada de la misma; de dos nuevos caminos de comunicación con estas islas, uno por un estrecho que llaman de Anian (Bering), que comunica Nueva España con China, a 80 leguas al oeste de la Punta de Bacalaos (Terranova), y el otro por Nuevo México, en altura de 45 grados. Manila, 27 de Junio de 1597. Con duplicado. Mapa de las islas de Luzón y Hermosa y parte de la costa de la China, por Fernando de los Ríos Coronel. AGI, Filipinas, 18B, R.7, N.68.

155 Campanella (1991) 154 (correspondiente a la Monarquía de España).

156 Agustín de Hipona (2011) 82. 
tions of time - a collection of eras, seasons and periods - and space - a collection of domains, territories, climates and Orients - were preserved, or at least developed within their own parameters. ${ }^{157}$

Having reached thus far, we can state that the 'Chinese histories' are nothing other than a negative of the 'Castilian and Portuguese histories', whose principal foundation is none other than the bulls. In short, and insofar as it established a year zero, the uti possidetis cancels the time preceding the Alexandrine bulls, which nevertheless assumed self-assigned powers to distribute rights, but not territories. We could say that this was the mission of the treaties, from the first signed at Tordesillas to the last, signed just before the collapse of the Catholic Monarchy, although even these acted on spaces partly uncontrolled, partly unknown, to the extent that this medieval logic was not merely inherited by the American states after independence, but it also justified the territorial expansion that followed.

The above-mentioned expansion had its origins in another significant date, namely that of 1810 , that served to fix another 'year zero' regarding the uti possidetis: independence. However, as we know, most of the American territories were not fully emancipated in 1810, and in many of them there remained in force the Constitution of the Spanish Monarchy of 1812, whose implementation brought about a major territorial reform based on a new conception of political representation. ${ }^{158}$ Indeed, the earliest Hispanic Constitutions promoted a fully-fledged municipal revolution, which continued to weigh on practically all the emancipated states throughout the $19^{\text {th }}$ century. ${ }^{159}$ Although initially it may appear not to be, this issue is linked to the uti possidetis, since throughout Central America, that is, in the territory of the ancient Audiencia of Guatemala today divided into different states, the accepted date for resolving territorial conflicts is 1821 , identified with the date of Independence. Given this state of affairs, the "last" demarcation by the Monarchy was made in constitutional times, independently of whether it coincided or not with the previous legacy. ${ }^{160}$ It is interesting to observe that the date of 1810 cancels the American constitutional time governed by the first Constitution of the Spanish Monarchy, which is not usually taken into

157 Granet (2013) 79.

158 Lorente / Portillo (2012).

159 AnNino (1995) and (2008).

160 LORENTE (2008). 
account with regard to the uti possidetis. Ultimately, 1810 is the result of a nationalist logic that for decades has not deigned to include the text of the Constitution of 1812 in the compilations of historical constitutions of American states. Needless to say, this logic is not exclusive to Latin America, as can be seen from the persistent denial of the "Spanishness" of the Bayona text, but exclusions of this kind have ceased to affect historiography: ${ }^{\mathbf{1 6 1}}$ as far as I am aware, they only endure in the realm of the uti possidetis.

\subsection{From Corporative Monarchy to National State}

«The ground upon which the community State is erected, considered from its juridical aspect, is the space in which the power of the state can exercise its specific activity, namely public power", affirms Jellinek in his well-known General Theory of the State. ${ }^{162}$ I will not expound here on the architects of nineteenth-century juridical science; ${ }^{163}$ I have only mentioned this quote from the German publicist in order to complete it with another taken from the same work: «The need of a territory, for a State to exist, has been recognized for the first time in modern times [...] None of the definitions of State that reach us from former times speak of the territory [...] Klüber is the first, as far as I know, to define the state as a civil society 'with a given territory'». ${ }^{164}$

Marchetti has explained in detail the absence of the territory in pre-modern juridical reflection: that is why the "territorial limits" were not definitively identified with the word 'border' until the $18^{\text {th }}$ and $19^{\text {th }}$ centuries. ${ }^{165}$ However, it appears that such assimilation was not reached by the Catholic Monarchy before its collapse, since, although in the Diccionario de Autoridades (1726-1739), 'border' is defined as "The line and boundary that separates and divides two Kingdoms, that are contiguous to each other", it described 'limit' as «The precinct, confine or boundary line of possessions, lands or state». ${ }^{166}$ However, perusing further in the Diccionario, we find the following surprise: 'state' was defined as «The present and conditional existence in which a thing is or is considered to be». ${ }^{\mathbf{1 6 7}}$ My aims in this paper do

161 Busaall (2011).

162 Jellinek (1981) 293.

163 Fioravanti (1979).

164 Fioravanti (1979) 296.

165 Febvre (1962).

166 Diccionario de Autoridades.

167 Diccionario de Autoridades. 
not include going through all the beads on the rosary for the pre-modern projection of the state; to the contrary, I shall merely mention the 'technical' consequences, as it were, of such a projection on the ambit of the uti possidetis in Latin America. One example will suffice: in Emile Loubet's award (11 September 1900), ${ }^{\mathbf{1 6 8}}$ the territory belonging to the United States of Colombia was described with reference to the following list of titles:

«Having made a minute and profound study of said instruments, to us submitted by the parties, especially of the royal cedulas of July 27, 1513, of September 6, 1521, of the royal provision of April 21, 1529, of the royal cedulas of March 2, 1537, of January 11, and May 9, 1541, of January 21, 1557, of February 23 and July 18, 1560, of August 4, and 9, 1561, of September 8, 1563, of June 28, 1568, of July 17, 1572, of the Capitulation of Pardo of December 1, 1573, of the Compilation of the Laws of the Indies of 1680, particularly of Laws IV, VI, IX of that compilation, of the royal cedulas of July 21, and November 13, 1722, of August 20,1729, of May 24, 1740, of October 31, 1742, of November 30, 1756, of the different instructions emanating from the Spanish Sovereign and addressed to the Superior Authorities of the Viceroyalty of Santa Fe as well as those of the Captaincy General of Guatemala in the course of the eighteenth century, and in the years following; of the royal orders of 1803 and 1805 , of the stipulations of the treaty concluded in 1825 between the two independent Republics, etc.» ${ }^{\mathbf{1 6 9}}$

As well as lengthy, the quotation is heavy; nevertheless, this adjective aptly describes the legislation of the Catholic Monarchy viewed from the perspective of today. In the mentioned arbitral award, royal cedulas, compilations of laws, royal orders and even instructions of all descriptions, are accumulated, and I am afraid to say, contradict each other without any regard for chronology, rank, or even authority. At the time, the coherence of this complex normative order was assured by the jurisprudential nature of the pre-modern legal order; today, however, the loss of context confers a diametrically opposite meaning to those texts. ${ }^{170}$ It has been said that one of the problems intrinsic to the administration of the uti possidetis is its projection across the territory affected by the provisions, as on many occasions these are unclear or aimed at spaces unknown in part or in full; all the same, it is not the provisions themselves that are found troublesome, whichever they may be. So we can only conclude that the uti possidetis does not aim to resuscitate the

168 ANDERSON (1911).

169 Vásquez Carrizosa (1974) 185.

170 Garriga (2007). 
interpretative framework of the past, but to transform its current administrators into the new interpreters of the regulations in question.

In this parable, legal history is lost, despite which it becomes the legitimating premise par excellence of the whole operation. That is why the historiography that points out the jurisdictional and corporative nature of the political order under the Catholic Monarchy, has no place in the narratives on territory that are regularly used in the border conflicts. Note that said historiography is not exclusively legal; to the contrary, some of the best work on questions of this kind were written by historians of America, ${ }^{171}$ increasingly convinced that critical legal history provides them with very useful tools for their work.

To all this we can add that the Hispanic world in the $18^{\text {th }}$ century did not witness the collapse of traditional juridical culture, ${ }^{\mathbf{1 7 2}}$ since this issue contains another that is highly relevant for the purposes of uti possidetis. The preferred demarcations in Latin American territorial disputes were the last to be drawn by the Monarchy. This is why in the majority of cases the most relevant provisions are those dictated during that century and up to 1810 . During those years, a new plan was assigned to the kingdoms of the Indies with the founding of the famous Intendencies that strove to redefine the American space. Besides the difficulty or impossibility of their implementation, the dividing lines between intendencies were drawn upon those of the earliest times, to which we must add that the defensive militarization process that reached its peak during the ministry of Godoy failed to clarify spacerelated issues but, instead, added considerably to the confusion. As Lempérière has explained with great clarity, throughout the $18^{\text {th }}$ century the number of corporations, and therefore of fueros, increased exponentially, to the extent that conflicts over jurisdiction dominated the scene in the government of the Indies. ${ }^{173}$

In this state of affairs, should the complex American jurisdictional fabric be recovered, the consequences for the uti possidetis would be, owing to their absurdity, disastrous, especially when the territory under dispute is maritime; a single example will illustrate this statement. The coastline of part of

171 LÉMPERIÈre (2004) and (2003).

172 Garriga (2002).

173 Martínez (2007). 
present-day Honduras was considered the jurisdiction of the Captaincy General of Cuba, owing to the fact that what today are territorial waters of Honduras could only be controlled from Havana. Hence, the strict application of uti possidetis would entail the extension of Cuba's territorial waters up to the Honduran coastline, which squarely contradicts the basic principles of international law. Nevertheless, a similar argument has been wielded for over a century in Colombian disputes, whose governments have repeatedly used a royal cedula, dated in 1803, according to which the islands of San Andrés and the portion of the coast of Mosquitos which extends from Cape Gracias a Dios inclusive, as far as the river Chagres, were segregated from the Captaincy General of Guatemala and dependent of the Viceroyalty of Santa Fe. In rigour, the term "dependent" should be subjected to examination, but everything seems to indicate that the objectives of the parties involved in the conflict did not include striving to recover the historical significance of the language used by the Catholic Monarchy. ${ }^{174}$

All the above allows us to state that the drive to "upgrade" the old jurisdictional borders by identifying them with those of the state, was, at the very least, an impossible task.

\section{Recapitulation}

Uti possidetis ita possideatis, 'as you possess, thus may you possess'. It would seem that the present times are not particularly propitious to recurring to Roman law once more with a view to justifying or building our current law. However, the use of the Latin expression to name the principle of international law almost inevitably obliges scholars to mention, at least, its original meaning. Some place emphasis on the archaic nature of the expression, relating it to the territories occupied by armies in the course of a conflict; ${ }^{175}$ others, by contrast, focus on analysing the possessory interdict, forcing its meaning to make it coincide with the intelligence available today. However, few have attempted to raise the question of ownership, despite the fact that the meaning of the uti possidetis in Latin American disputes is closer to a

174 Libro blanco de la República de Colombia (1980) (available at: http://www.sogeocol. edu.co/documentos/Lib_Blanco.pdf).

175 http://www.oxfordreference.com/view/10.1093/acref/9780195369380.001.0001/acref9780195369380. 
claim for ownership over a territory than the provisional protection of the possessor. This is why I have amended the expression to bring it closer to the current situation.

"Uti possidetis ita domini eritis". Any observer would claim that this amendment only takes us to the sphere of prescription, which to a certain extent is true. However, the problem lies not so much in the condition of the prospective owner as in the object that is possessed, which in the case in hand are titles and not physical spaces. This is where the perverse logic consubstantial with the uti possidetis comes into play, since it does not refer to the history of the territory to resolve border disputes, but to the contrary, it creates that history, and projects it upon the disputed territory.

\section{Bibliography}

Velasco (1841-1844) (Juan de Velasco completed this work in 1789; I have used the edition published in Quito, Casa de la Cultura Ecuatoriana, 1977, available at: http://www.bibliotecayacucho.gob.ve/fba/index.php?id=97\&backPID=103\& begin_at $=72 \&$ tt_products $=82$ )

Abulafia, David (2009), El descubrimiento de la humanidad. Encuentros atlánticos en la era de Colón, Barcelona: Crítica

Agüero, Alejandro (2006), Las categorías básicas de la cultura jurisdiccional, in: Lorente, Marta (coord.), De la justicia de jueces a la justicia de leyes: Hacia la España de 1870, Madrid: Consejo General del Poder Judicial, 19-58

Agüero, Alejandro (2008), Castigar y perdonar cuando conviene a la República. La justicia penal de Córdoba del Tucumán, siglos XVII y XVIII, Madrid: Centro de Estudios Políticos y Constitucionales

Aguilar Rivera, José Antonio (2012), Ausentes del universo. Reflexiones sobre el pensamiento político hispanoamericano en la era de la construcción nacional, 1821-1850, México: FCE

Aguilar Rivera, José Antonio, Rafael Rojas (2002), El republicanismo en Hispanoamérica: Ensayos de historia intelectual y política, México: FCE

Agustín de Hipona (2011), Qué es el tiempo. Libro XI de las Confesiones, (bilingual edition and translation by Agustín Corti), Madrid: Trotta

Alcalá Zamora, Niceto (1944), Nuevas Reflexiones sobre las Leyes de Indias, Buenos Aires: Ed. Kraft

Álvarez, Alejandro (1909), Latin America and International Law, in: The American Journal of International Law 3, 2, 69-353 (available at: http://dx.doi.org/ $10.2307 / 2186127)$ 
Álvarez Jiménez, Alberto (2011), Private Rights, the User of Maps, State Responsibility, and Other Issues in the International Court of Justice's Boundary Jurisprudence: 2000-10, in: The Canadian Yearbook of International Law 49, 1, $177-211$

Anderson, Benedict, (2001), Nacionalismo oriental y nacionalismo occidental. ¿ڤHay alguna diferencia importante?, in: New Left Review 9, 70-80 (available at: http://newleftreview.es/article/download_pdf?language=es\&id=2320)

Anderson, Luis (1911), El laudo Loubet: contribución al estudio de la cuestión de límites entre Costa Rica y Panamá, San José, Costa Rica: Tip. de A. Alsina

Anghie, Antony (1996), Francisco de Vitoria and the Colonial Origins of International Law, in: Social and Legal Studies 5, 3, 321-336

Anghie, Antony (1999), Finding the Peripheries: Sovereignty and Colonialism in Nineteenth-Century International Law, in: Harvard International Law Journal $40,1-80$

Anghie, Antony (2005), Imperialism, Sovereignty and the Making of International Law, Cambridge, U. K.: Cambridge University Press

Anghie, Antony (2006), The Evolution of International Law: colonial and postcolonial realities, in: Third World Quarterly 27, 5, 739-753

Annino, Antonio (1995), Cádiz y la revolución territorial de los pueblos mexicanos, in: Annino Antonio (coord.), Historia de las elecciones en Iberoamérica, Siglo XIX, Buenos Aires: FCE, 177-226

Annino, Antonio (2008), México, ¿soberanía de los pueblos o de la nación?, in: García Clara, Francisco Sales (eds.), Reflexiones en torno a los centenarios: los tiempos de la Independencia, México DF: Centro de Investigación y Docencia Económicas / Centro de Estudios Sociales y Opinión Pública, 37-54

Armitage, David (2007), The Declaration of Independence: A Global History, Cambridge, Massachusetts: Harvard University Press

Bandeira Galindo, George Rodrigo (2005), Martti Koskenniemi and the Historiographical Turn in International Law, in: The European Journal of International Law 16, 539-559

Bandeira Galindo, George Rodrigo (2012), Force Field: On history and Theory of International Law, in: Rechtsgeschichte 20, 86-103

Barrientos Grandón, Javier (2000), Historia del Derecho Indiano. Del descubrimiento colombino a la codificación. I. Ius commune - ius proprium en las Indias occidentales, Roma: Il Cigno Galileo Galilei

Barrientos Grandón, Javier (2001), Guía prosopográfica de los ministros togados de Indias (1503-1898), Madrid: Fundación Histórica Tavera, CD-Rom, Derecho y Justicia en Iberoamérica

Barrientos Grandón, Javier (2004), El cursus de la jurisdicción letrada en las Indias (s. XVI-XVII), in: Barrios, Feliciano (coord.), El Gobierno de un Mundo: Virreinatos y Audiencias en la América Hispánica, Cuenca: Universidad de Castilla-La Mancha, 633-708 
Becker Lorca, Arnulf (2006a), Alejandro Álvarez Situated: Subaltern Modernities and Modernism that Subvert, in: Leiden Journal of International Law 19, 879-930

Becker Lorca, Arnulf (2006b), International Law in Latin America or Latin America International Law? Rise, Fall, and Retrieval of a Tradition of Legal Thinking and Political Imagination, in: Harvard International Law Journal 47, 1, 283-305

Becker Lorca, Arnulf (2010), Universal International Law: Nineteenth-Century Histories of Imposition and Appropriation, in: Harvard International Law Journal 51, 2, 475-552

Beneyto, José María, David Kennedy (2012), New Approaches to International Law, The Hague: Asser Press

Brading, David A. (1991), Orbe indiano. De la monarquía católica a la república criolla, 1492-1867, México: Fondo de Cultura Económica

Bravo Lira, Bernardino (1996), Por la razón o la fuerza: el estado de derecho en la historia de Chile, Santiago de Chile: Eds. Univ. Católica de Chile

Bruce St. John, Ronald (1998-1999), Ecuador-Perú Endgame, in: Boundary and Security Bulletin 6, 4, 79-85

Busaall, Jean-Baptiste (2011), Le spectre du jacobinisme. L'expérience constitutionnelle française et le premier libéralisme espagnol (1808-1814), Madrid: Casa de Velázquez

Calderón, María Teresa, Clément Thibaud (2010), La Majestad de los Pueblos, Nueva Granada y Venezuela, 1780-1832, Bogotá: Taurus

Calvo, Carlos (1862), Colección completa de los tratados, convenciones, capitulaciones, armisticios y otros actos diplomáticos de todos los estados de América Latina, Paris: Libreria de A. Durand

Campanella, Tommaso (1991), La política, Madrid: Alianza

Carmagnani, Marcello (1988), El regreso de los dioses. El proceso de reconstitución de la entidad étnica en Oaxaca, siglos XVII y XVIII, Mexico: FCE

Carty, Anthony (1991), Critical International Law: Recent Trends in the Theory of International Law, in: European Journal of International Law 2-1, 1-27 (available at: http://www.ejil.org/article.php?article=2026\&issue=101)

Castellino, Joshua (2008), Territorial Integrity and the "Right" to Self-Determination: An Examination of the Conceptual Tools, in: Brook. Journal of International Law 33, 2, 499-567

Castellino, Joshua, Steve Allen (2003), Title to Territory in International Law. A Temporal Analysis, Aldershot: Dartmouth Publishing Company

Castro Leiva, Luis (1991), De la patria boba a la teología bolivariana, Caracas: Monte Ávila

Chakrabarty, Dipesh (2008), Al margen de Europa. ¿Estamos ante el final del predominio cultural europeo?, Barcelona: Tusquets Editores

Chiaramonte, José Carlos (1997), Ciudades, provincias, estados. Orígenes de la Nación Argentina, 1800-1846, Argentina: Compañía Editora Espasa Calpe Argentina / Ariel 
Chiaramonte, José Carlos (2004), Nación y Estado en Iberoamérica. El lenguaje político en tiempos de la independencia, Buenos Aires: Editorial Sudamericana

Clavero, Bartolomé (2005), Freedom's Law and Indigenous Rights. From Europe’s Oeconomy to the Constitutionalism of the Americas, Berkeley: University of California-Robbins Collection

Colombani, Jean-Marie (2012), China quiere la primacía. El XIX fue el siglo de la humillación; el XX, el de la restauración; y el XXI, el del dominio, in: El País, 19,11

Constitución del Estado de Cartagena de Indias sancionada por la Convención General en 14 de Junio de $1812,2^{\circ}$ de la Independencia, Cartagena de Indias, Imprenta del C. Diego Espinosa, MDCCCXIII

Costa, Pietro (1989), Saperi, disciplina, disciplinamento: verso una 'nuova' storia della cultura giuridica?, in: Annali della Facoltà di Giurisprudenza (Università degli Studi di Macerata. Nuova serie). Filosofia del Diritto e Teoria Politica II, 993-1027

De la Rasilla Moral, Ignacio (2013), El estudio del Derecho Internacional en el corto siglo XIX español, in: Rechtsgeschichte 21, 48-65

De Vel-Palumbo, Fabien (2008), Using the Past to Serve the Present: The 'Century of Humiliation' and Political Legitimacy in 21st Century China, Adelaide, University of Adelaide, School of History and Politics, Discipline of Politics, International Studies

Dispute between Argentina and Chile concerning the Beagle Channel, Decision of 18 February 1977, in: Reports of International Arbitral Awards XXI, 57-223

Durán Bachler, Samuel (1972), The Latin American Doctrine of Uti Possidetis, Genève: Institut Universitaire de Hautes Études Internationales

Durán Bachler, Samuel (1989), Resurrección del uti possidetis, in: Revista Chilena de Derecho 16, 489-491

Dzurek, Daniel (1996), The Spratly Islands dispute: Who's on first? Durham, U. K.: International Boundaries Research Unit, University of Durham (available at: http://www.nguyenthaihocfoundation.org/lichsuVN/SpratlyDispute.pdf)

Esquirol, Jorge L. (2011), The Latin American Tradition of Legal Failure, in: Comparative Law Review 2, 1, 1-19

Exposición Pacífico. España y la aventura de La Mar del Sur (exhibition catalog) (2013), Madrid: Ministerio de Educación, Cultura y Deporte

Fassbender, Bardo, Anne Peters (eds.) (2012), The Oxford Handbook of the History of International Law, Oxford: OUP Oxford (Oxford Handbooks in Law)

Febvre, Lucien (1962), Frontière: le mot et la notion, in: Febvre, Lucien, Pour une histoire à part entière, Paris: SEVPEN, 11-24

Fioravanti, Maurizio (1979), Giuristi e costituzione politica nell'Ottocento tedesco, Milano: Giuffrè

Florescano, Enrique (1994a), Memoria Mexicana, México: FCE

Florescano, Enrique (1994b), La concepción nahua del tiempo y del espacio, in: Memoria mexicana, México: FCE, 100-133 
Foucault, Michel (1976), Questions à Michel Foucault sur la Géographie, in: Herodote $1,71-85$

Foucault, Michel (1984), Des espaces autres. Hétérotopies, in: Architecture, Mouvemente, Continuité, 5 (available at: http://desteceres.com/heterotopias.pdf)

Garriga, Carlos (2002), Los límites del reformismo borbónico: a propósito de la administración de la justicia en Indias, in: Barrios, Feliciano (coord.), Derecho y Administración Pública en las Indias hispánicas. Actas del XII Congreso Internacional de Historia del Derecho Indiano (Toledo, 19-21 October 1998), Cuenca: Universidad de Castilla-La Mancha, v. I, 781-821

Garriga, Carlos (2004a), Orden jurídico y poder político en el Antiguo Régimen, in: Istor. Revista de historia internacional 16, 13-44

Garriga, Carlos (2004b), Las Audiencias: la justicia y el gobierno de las Indias, in: Barrios, Feliciano (coord.), El Gobierno de un Mundo: Virreinatos y Audiencias en la América Hispánica, Cuenca: Universidad de Castilla-La Mancha, 711-794

Garriga, Carlos (2006), Patrias criollas y plazas militares, in: Martiré, Eduardo (coord.), La América de Carlos IV, (I), Buenos Aires: Instituto de Investigaciones de Historia del Derecho, 35-130

Garriga, Carlos (2007), Justicia animada. Dispositivos de la justicia en la Monarquía católica, in: Lorente, Marta (coord.), De justicia de jueces a justicia de leyes: hacia la España de 1870, Madrid: Consejo General del Poder Judicial, 59-104

Gaudemet, Jean (1996), L'arbitrage dans les conflits territoriaux entre cités dans l'Antiquité gréco-romaine: le principe du 'statu quo' et l'uti possidetis, in: L'assistance dans la résolution des conflits, (Recueils de la Société Jean Bodin pour l'histoire comparative des institutions), Bruxelles: Edition de Boeck Université, v. 1, 37-46

Gerbi, Antonello (1960), La disputa del nuevo mundo. Historia de una polémica, 1750-1900, México: FCE

Gozzi, Gustavo (2010), The Particularistic Universalism of International Law in the Nineteenth Century, in: Harvard International Law Journal-Online 51 (available at: http://www.harvardilj.org/2010/10/online_52_gozzi/)

Granet, Marcel (2013), El pensamiento chino, Trotta: Madrid

Gruzinski, Serge (1991), La colonización de lo imaginario. Sociedades indigenas y occidentalizaciôn en el Mexico español. Siglos XVI-XVII, Mexico: FCE

Gutiérrez Ardila, Daniel (2010), Un nuevo reino. Geografía política, pactismo y diplomacia durante el interregno en Nueva Granada (1808-1816), Bogotá: Universidad Externado

Halperin Donghi, Tulio (1985), Reforma y disolución de los Imperios ibéricos, 1750-1850, Madrid: Alianza Editorial

Harley, John Brian, David Woodward (eds.) (1994), Cartography in the Traditional East and Southeast Asian Societies, in: The History of Cartography, Volume 2, University of Chicago Press (available at: http://www.press.uchicago.edu/ books/HOC/index.html) 
Hasani, Enver (2003), Uti Possidetis Juris: From Rome to Kosovo, in: The Fletcher Forum of World Affairs 27, 2, 85-97

Hays Gries, Peter (2004), China's New Nationalism: Pride, Politics and Diplomacy, Berkeley: University of California Press

Hébrard, Véronique (1997), Une nation par le discours: le Venezuela (1808-1830), Paris: l'Harmattan

Hensel, Paul R., Michael E. Allison (2004), The Colonial Legacy and Border Stability: Uti Possidetis and Territorial Claims in the Americas (available at: http://www.paulhensel.org/Research/io05.pdf)

Hespanha, Antonio M. (1993a), El espacio político, in: La Gracia del Derecho. Economía de la cultura en la Edad Moderna, Madrid: CEC, 85-121

Hespanha, Antonio M. (1993b), Incursión en el pensamiento jurídico político chino, in: La gracia del derecho. Economía de la cultura en la edad moderna, Madrid: CEC, 275-293

Hespanha, Antonio M. (1996), Cultura Jurídica Europeia. Síntese de um milénio, Lisboa: Fundaçâo Boiteux

Historical Evidence To Support China's Sovereignty over Nansha Islands (available at: http://www.fmprc.gov.cn/eng/topics/3754/t19231.htm.)

Hobson, John M. (2006), Los orígenes orientales de la civilización de Occidente, Barcelona: Crítica

HuI, WANG (2006), El pensamiento chino contemporáneo y la cuestión de la modernidad, in: Istor, 25, 27-72 (available at: http://www.istor.cide.edu/archivos/ num_26/dossier2.pdf)

International Crisis Group, 'Dangerous waters: China-Japan relations on the rocks' (2013), in: Asia Report, 245, 1-59 (available at: http://www.crisisgroup.org/ / media/Files/asia/north-east-asia/245-dangerous-waters-china-japan-relations-onthe-rocks.pdf)

Ireland, Gordon (1940), Conflictos de límites y de posesiones en Sudamérica, Buenos Aires: Escuela de Guerra Naval

Island of Palmas case (Netherlands, USA), 4 April 1928 (2006), in: Reports of International Arbitral Awards, t. II, 829-871 (available at: http://legal.un.org/riaa/ cases/vol_II/829-871.pdf)

Jellinek, George (1981), Teoría general del Estado, translation from German of the second edition and prologue by Fernando de los Ríos, Buenos Aires: Ed. Albatros

Jennings, Robert Yewdall (1963), The Acquisition of Territory in International Law, Manchester: Manchester University Press

Jie, Chen (1994), China's Spratly Policy: With Special Reference to the Philippines and Malaysia, in: Asian Survey 34, 10, 893-903

Johnston, Douglas M., Mark J. Valencia (1991), Pacific Ocean Boundary Problems: status and solutions, Dordrecht: Martinus Nijhoff Publishers

Kaplan, Robert D. (2013), La venganza de la geografía. Cómo los mapas condicionan el destino de las naciones, Barcelona: RBA Libros 
Kennedy, David (1980), Toward a Historical Understanding of Legal Consciousness: the Case of Classical Legal Thought in America 1850-1940, in: Research in Law and Sociology 3, 3-24

Kennedy, David (1988), A New Stream of International Law Scholarship, in: Wisconsin International Law Journal 7, 1-49

Kennedy, David (1999), International Law and the Nineteenth Century: History of an Illusion, in: Harvard Law Review 17, 100, 99-132 (available at: http:// www.law.harvard.edu/faculty/dkennedy/publications/illusions.pdf)

Kohen, Marcelo G. (1997), Possession contestée et souveraineté territorial, Paris: P.U.F.

Kohen, Marcelo G. (2001), La contribución de América Latina al desarrollo progresivo del derecho internacional en materia territorial, in: Anuario de Derecho Internacional XVII, 57-77

Kohen, Marcelo G. (2009), La contribution de l'Amérique latine au développement progressif du droit international en matière territoriale, in: Relations Internationales 139, 13-29

Koskenniemi, Martti (1997), Lauterpacht: The Victorian Tradition in International Law, in: European Journal of International Law 2, 215-263

Koskenniemi, Martti (2011), Histories of International Law: Dealing with Eurocentrism, in: Rechtsgeschichte 19, 152-176 (available at: http://dx.doi.org/ $10.12946 / \mathrm{rg} 19 / 152-176)$

Koskenniemi, Martti (2004), Why History of International Law Today?, in: Rechtsgeschichte 4, 61-66 (available at: http://dx.doi.org/10.12946/rg04/061066)

Koskenniemi, Martti (2004b), The Gentle Civilizer of Nations. The Rise and Fall of International Law, 1870-1960, Cambridge: Cambridge University Press

Koskenniemi, Martti, Colonization of the 'Indies' The Origin of International Law? (conference given in Zaragoza, December 2009, now available at: http:// ifc.dpz.es/recursos/publicaciones/30/12/05koskenniemi.pdf)

Land, Island and Maritime Dispute (El Salvador/Honduras: Nicaragua intervening), Judgment of 11 September 1992, I. C. J. Reports 1992

Landavazo, Marco Antonio (2001), La máscara de Fernando VII. Discurso e imaginario monárquico en una época de crisis. Nueva España, 1808-1822, México: El Colegio de México/Universidad Michoacana de San Nicolás de Hidalgo/El Colegio de Michoacán

Lempérière, AnNick (2003), De la república corporativa a la nación moderna (1821-1860), in: Annino Antonio, François-Xavier Guerra (coords.), Inventando la Nación. Iberoamérica. Siglo XIX, México: Fondo de Cultura Económica, 316-346

Lempérière, Annick (2004), Entre Dieu et le Roi, la République. Mexico, XVI ${ }^{\mathrm{e}}-\mathrm{XIX}{ }^{\mathrm{e}}$ siècles, Paris: Les Belles Lettres

Lempérière, AnNick (2005a), La «question colonial», Nuevo Mundo Mundos Nuevos. Débats, mis en ligne le 08 février 2005 (available at: http://nuevomundo. revues.org/437) 
Lempérière, ANNick (2005b), ¿Nación moderna o república barroca? México 1823-1857, in: Nuevo Mundo Mundos Nuevos (available at: http://nuevo mundo.revues.org/index648.html)

Lesaffer, Randall (2005), Argument from Roman Law in Current International Law: Occupation and Acquisitive Prescription, in: The European Journal of International Law 16, 1, 25-58

Levaggi, Abelardo (2002), Diplomacia hispano-indígena en las fronteras de América: Historia de los tratados entre la Monarquía española y las comunidades aborígenes, Madrid: Centro de Estudios Políticos y Constitucionales

Levathes, Louis (1997), When China Ruled the Seas: The Treasure Fleet of the Dragon Throne, 1405-1433, Oxford: Oxford University Press

Lockart, James (1999), Los nahuas después de la Conquista. Historia social y cultural de la población indígena del México central, siglos XVI-XVIII, México: FCE

Lorente Sariñena, Marta (2008), El fracaso de la Intendencia en Honduras: la Alcaldía Mayor de Tegucigalpa (1799-1819), in: De la Puente Brunke, José, Jorge Armando Guevara Gil (coords.), Derecho, instituciones y procesos históricos: XIV Congreso del Instituto Internacional de Historia del Derecho Indiano, Lima: Pontificia Universidad Católica del Perú, Instituto Riva Agüero, v. II, 567-590

Lorente Sariñena, Marta, José M. Portillo (dirs.) (2012), El momento gaditano. La Constitución en el orbe hispánico, 1808-1826, Madrid: Congreso de los Diputados

Lowenthal, David (1998), El pasado es un país extraño, Madrid: Akal

Marchetti, Paolo (2001), De iure finium. Diritto e confini tra tardo medievo ed età moderna, Milano: Giuffrè

Martínez, Fernando (2007), Estrépito de Tribunales. Competencias de jurisdicción en la América de Carlos IV, in: Martiré, Eduardo (coord.), La América de Carlos IV, (I), Buenos Aires: Instituto de Investigaciones de Historia del Derecho, 11-96

Meccarelli, Massimo (1998), Arbitrium. Un aspetto sistematico degli ordinamenti giuridici in età di diritto comune, Milano: Giuffrè

Meglio, Gabriel di (coord.) (2008), Lo revolucionario en las revoluciones de independencia iberoamericana (Dossier de la revista Nuevo Topo), in: Revista de Historia y pensamiento crítico 3, 1-6 (available at: http://historiapolitica.com/ datos/biblioteca/xix2dimeglio.pdf)

Molina, Raul A. (1955), Misiones argentinas en los archivos europeos, México: Instituto Panamericano de Geografía e Historia

Morelli, Federica (2001), Territorio o Nazione. Riforma e dissoluzione dello spazio imperiale in Ecuador, 1765-1830, Soneria Mannelli: Rubbettino

Nesi, Giuseppe (1996), L'uti possidetis iuris nel diritto internazionale, Padova: Cedam

Nuzzo, Luigi (2008), De Italia a las Indias. Un viaje del derecho común, in: Revista Estudios Socio-Jurídicos 10, 1, 87-126 
Nuzzo, Luigi, Miloš Vec (eds.) (2012), Constructing International Law. The Birth of a Discipline, Frankfurt am Main: Vittorio Klostermann

O’Gorman, Edmundo (1986), La supervivencia política novo-hispana. Monarquía o república, México: Universidad Iberoamericana

Obregón, Liliana (2002), Completing Civilization: Nineteenth Century Criollo Interventions in International Law, Cambridge: Harvard Law School (unpublished doctoral thesis)

Obregón, Liliana (2006), Between Civilisation and Barbarism: Creole interventions in international law, in: Third World Quarterly 27, 5, 815-832

Obregón, Liliana(2009), Construyendo la región americana: Andrés Bello y el derecho internacional, in: González-Stephan, Beatriz, Juan Poblete (eds.), Andrés Bello y los estudios latinoamericanos, Pittsburgh: Universidad de Pittsburgh: Instituto Internacional de Literatura Iberoamericana, 65-86

Ollé, Manel (2002), La empresa de China. De la armada invencible al Galeón de Manila, Barcelona: El Acantilado

Özsu, Umut (2010), Agency, Universality, and the Politics of International Legal History, in: Harvard International Law Journal-Online, 51 (available at: http://www.harvardilj.org/?s=umut+0C3\%B6zsu)

Padgen, Anthony (1991), El imperialismo español y la imaginación política, Barcelona: Planeta

Padgen, Anthony (1997), Señores de todo el mundo. Ideologías del Imperio en España, Inglaterra y Francia (en los siglos XVI, XVII y XVIII), Barcelona: Península

Palacios, Guillermo (coord.) (2009), La nación y su historia. Independencias, relato historiográfico y debates sobre la Nación: América Latina, siglo XIX, México: El Colegio de México

Peralta, Manuel María (1886), Límites de Costa Rica y Colombia (1573-1881), Madrid: Librería de M. Murillo

Pérez Bustamante, Ciriaco (1922), "La Bula de Alejandro VI y el meridiano de demarcación. Portugueses y españoles en Oceanía. La expedición de López de Villalobos”. Discurso leído en la Sección Universitaria de Canarias en la solemne apertura del curso académico de 1922 a 1923, La Laguna: Imprenta de suc. de M. Curbelo, 1-59

Podgorny, Irina (2011), Fronteras de papel: archivos, colecciones y la cuestión de límites en las naciones americanas, in: Historia Crítica 44, 56-79

Portillo, José M. (2006), Crisis atlántica. Autonomía e independencia en la crisis de la monarquía española, Madrid: Fundación Carolina / Marcial Pons

Real Academia Española, Diccionario de Autoridades, Madrid: Real Academia Española (available at: http://web.frl.es/DA.html)

Remiro Brotóns, Antonio (1999), Universalismo, Multilateralismo, Regionalismo y Unilateralismo en el Nuevo Orden Internacional, in: Revista Española de Derecho Internacional 51, 111-58

Reports of International Arbitral Awards, t. II, 829-871 (available at: http://legal. un.org/riaa/cases/vol_II/829-871.pdf). 
Restrepo Piedrahita, Carlos (1996), Primeras Constituciones de Colombia y Venezuela, 1811-1830, $2^{\text {nd }}$ ed., Bogotá: Universidad Externado de Colombia

Rıвот, Luis et al. (coords.) (1995), El Tratado de Tordesillas y su época, 3 vv., España: Junta Castilla y León

Rodríguez, Ordoñez, Edmundo Jaime (2008), La independencia de la América española, México DF: Fondo de Cultura Económica

Roux, Jean Claude (2001), De los límites a la frontera: o los malentendidos de la geopolítica amazónica, in: Revista de Indias 223, 513-539

Samuels, Marwyn (1982), Contest for the South China Sea, New York: Methuen

San Miguel Casisa, Sara Roxana (2013), Soberanía chilena en la Antártica: desafíos y perspectivas actuales, Santiago de Chile, (available at: http://repositorio. uchile. cl/handle/2250/115320)

Sánchez Bella, Ismael (1980), Quito, Audiencia subordinada, in: Anuario Histórico Jurídico Ecuatoriano V, 3-47

SÁNChez Rodríguez, Luis (1988), “Uti possidetis”: la reactualización jurisprudencial de un viejo principio, in: Revista española de derecho internacional 40, 121-152

SÁnchez Rodríguez, Luis (1993), Problemas de fronteras en América: las fronteras terrestres entre países iberoamericanos, in: Araceli Mangas, Martín (ed.), La Escuela de Salamanca y el derecho internacional en América. Del pasado al futuro, Salamanca: Jornadas Iberoamericanas de la Asociación de Profesores de Derecho Internacional, 93-115

Schmitt, Carl (1979), El nomos de la tierra en el Derecho de Gentes del Jus Publicum Europaeum, Madrid: CEC

SChröter, BERND (2001), La frontera en Hispanoamérica colonial: un estudio historiográfico comparativo, in: Colonial Latin American Historical Review 10, 3, 351-386

Segura Munguía, Santiago (2007), Frases y expresiones latinas de uso actual con un anexo sobre las instituciones jurídicas romanas, Bilbao: Universidad de Deusto

Semana (2012), 19/11/12 Colombia "rechaza" partes del fallo sobre límites con Nicaragua (available at: http://www.semana.com/nacion/articulo/colombia-rechaza -apartes-del-fallo-sobre-limites-nicaragua/268107-3)

Shaw, Han-Yi (1999), The Diaoyutai/Senkaku Islands dispute: its history and an analysis of the ownership claims of the P.R.C., R. O.C., and Japan, Baltimore: University of Maryland School of Law

Shaw, Malcolm N. (1996), The Heritage of States: The Principle of Uti Possidetis Juris Today, in: BYBIL XVII, 75-154

Shen, Jianming (1997), International Law Rules and Historical Evidence Supporting China's Tide to the South China Sea Islands, in: Hastings International and Comparative Law Review 40, 1-75

Shen, Jianming (2002), China's Sovereignty over the South China Sea Islands: A Historical Perspective, (available at: http://chinesejil.oxfordjournals.org/ content/1/1/94.full.pdf) 
Souza Sá Vianna, Manoel De (1912), De la non existence d'un droit International américain: dissertation présentée au Congres scientifique Latino-Américain, Rio de Janeiro: L. Figueredo

TAu AnzoÁtegui, Víctor (1992), Casuismo y sistema: indagación histórica sobre el espíritu del derecho indiano, Buenos Aires: Instituto de Investigaciones de Historia del Derecho

Tau Anzoategui, Víctor (1997), Nuevos Horizontes en el Estudio Histórico del Derecho Indiano, Buenos Aires: Instituto de Investigaciones de Historia del Derecho

Tau Anzoátegui, Víctor (2002), El Derecho Indiano y el trasplante de la cultura del Derecho común (siglos XVI y XVII), in: Cultura jurídica europea: una herencia persistente, Sevilla: Universidad Pablo de Olavide, 23-38

Terret, Steve (2000), The dissolution of Yugoslavia and the Badinter Arbitration Commission, Aldershot, U. K.: Ashgate / Dartmouth

Valencia, Mark J. (2007), The East China Sea dispute: context, claims, issues and possible solutions, in: Asian Perspective 31, 1, 127-167

Valencia, Mark J. (2010), Troubled waters: the search for maritime security in East Asia, in: Global Asia 5, 4, 8-17

Vásquez Carrizosa, Alfredo (1974), Quitasueño, Roncador, Serrana. Antecedentes históricos y jurídicos del Tratado entre la República de Colombia y los Estados Unidos deI 8 de Septiembre de 1972, Bogotá: Italgraf

Velasco, Juan De (1841-1844), Historia del Reino de Quito en la América Meridional, Quito: Imprenta del Gobierno

Vélez, Palmira (2008), Política e historiografía. El americanismo español hasta 1936, in: Revista de Indias LXVIII, 243-247

Verdò, Geneviève (2006a), El dilema constitucional en las Provincias Unidad del Río de la Plata (1810-1819), in: Historia contemporánea 33, 513-536

Verdò, Geneviève (2006b), L'indépendance argentine entre cités et nations (1808-1821), Paris: Publications de la Sorbonne

WANG, Dong (2005), China's Unequal Treaties: Narrating National History, Lanham: Md., Rowman \& Littlefield

WANG, Zheng (2012), Never Forget National Humiliation: Historical Memory in Chinese Politics and Foreign Relations: Columbia University Press

Weber, David J. (2007), Bárbaros. Los españoles y sus salvajes en la era de la Ilustración, Barcelona: Crítica

YeE, ANDY (2011), Maritime territorial disputes in East Asia: a comparative analysis of the South China Sea and the East China Sea, in: Journal of Current Chinese Affairs 2, 165-19

Zamora, Romina (2010), San Miguel de Tucumán, 1750-1812. La construcción social del espacio físico, de sociabilidad y de poder, in: Nuevo Mundo Mundos Nuevos [En ligne], Extraits de thèses, mis en ligne le 18 janvier 2010 


\section{Itineraries}



Paolo Cappellini

\section{Carl Schmitt revisited. Ripensare il Concetto di 'Grande Spazio' (Großraum) in un Contesto Globale*}

Quelle che si presentano qui, rispetto al programma delineato nel titolo ovvero prendere sul serio l'apporto del nostro autore ad una riflessione critica sul diritto internazionale quale si sta configurando in questa fase storica -, non sono e non possono essere altro che riflessioni preliminari. E per molti motivi.

Anzitutto si tratterebbe di superare una diffidenza di fondo, tanto più dura ad essere sfatata, in quanto fa leva su di una questione pregiudiziale di carattere morale, che chiama in causa la moralità stessa della scienza tout court e di quella giuridica in particolare.

Si tratta infatti di una fase del suo pensiero che molti considerano ancora indissolubilmente legata alla sua adesione al regime nazista. Saremmo allora in presenza - forzando non certo l'assonanza con la categoria oppositivamente e dialetticamente connessa di 'spazio economico' (Wirtschaftsraum), ${ }^{\mathbf{1}}$

* Questo saggio è dedicato alla memoria di un amico non dimenticato né dimenticabile, dai "tempi di Ferrara", Giovanni Battaglini.

1 Già nel 1942 Julius Evola aveva osservato il dislocarsi del concetto di grande spazio, che emergeva dapprima nell'“economia, con tratti abbastanza indeterminati”, per poi spostarsi nel campo della geopolitica, fino a giungere successivamente ad applicazioni già precise nella cd. teoria paneuropea del conte Coudenhove-Kalergi (587 col. I), in Evola (1942) che dopo aver analizzato la teoria dell'etnarchia di Carlo Costamagna, esprime un acuta valutazione della 'collocazione' della dottrina schmittiana: «Così, ad esempio, se si potrebbe ben pensare ad una etnarchia nel caso di un unico grande spazio controllato dalle potenze dell'Asse, dall'etnarchia si passerebbe alla direzione unitaria distinta ed autonoma dell'Italia e della Germania, quando si concepissero invece due grandi spazi, facenti capo all'una e all'altra nazione. Già con l'affrontare il problema della direzione si presenta, in ogni modo, quello della controparte politico-spirituale del "grande spazio" quale concetto soprattutto tecnico, geografico, economico. È merito di Carl Schmitt di aver criticata la concezione puramente neutra e tecnica del "grande spazio", affermando, di contro ad essa, l'idea, che l'elemento primario che in un "grande spazio" deve essere un Reich, cioè, più o meno, un impero. Il "grande spazio" sarebbe una conseguenza, in quantochè ogni impero ha bisogno di un "grande spazio": il quale allora andrebbe considerato più che come uno 
ma sì invece con quella pregiudicata, per non dir famigerata, di Lebensraum di un "garstiges Thema" (Joerges) o di un "belasteter Begriff" (Dreier). ${ }^{2}$

E questo tanto più, laddove si rifletta, per immediata evidenza, che essa è anche esplicitamente collegata con una 'disciplina' il cui statuto scientifico è stato fin dal principio controverso: appunto la Geopolitica.

È bensì vero che questa trovava tra i suoi più importanti corifei il geografo e geopolitico inglese Sir Halford J. Mackinder (1861-1947) con la sua "Hearthland-theory" (1904) e l'ammiraglio statunitense Alfred Thayer Mahan (1840-1914), ma nel momento in cui esce dall'ambito più strettamente politico-strategico e accademico per divenire "scienza applicata" con Karl Haushofer (1869-1946: Weltpolitik von heute, Zeitgeschichte-Verlag, Berlin 1934; Raumüberwindende Mächte, Teubner, Leipzig, Berlin 1934.), si incammina (via Rudolf Hess) a diventare poi, nei suoi rappresentanti più esposti, un aspetto, e non dei minori, di quella medesima cultura di regime; una etichetta difficile da strappare dall'immaginario, nonostante la tragica testimonianza di partecipazione alla resistenza contro Hitler di Albrecht Haushofer, figlio dell'autore della 'svolta' e geopolitico anch'egli. ${ }^{3}$

A fronte di questo doppio ostracismo varrà allora la pena di raccogliere una serie di elementi di riflessione.

Che, infatti, dopo la fine della "guerra fredda", la geopolitica sia tornata di attualità è per più versi altrettanto innegabile.

Si pensi, ad esempio, all'ormai risalente interesse di ricerca di un giurista e filosofo del diritto del rilievo di Mario G. Losano, che aveva trovato un momento significativo di emersione proprio in un saggio dedicato a I Grandi Spazi in un inedito progetto di trattato del 1943 fra gli Stati dell'Asse $(2006)^{4} \mathrm{e}$

spazio matematico-topografico, come un campo di forze, di influenze, di irradiazioni. Su tale base, è possibile andar ancor un passo avanti: dopo esser passati dalla idea di "spazio vitale" a quella, più impersonale, di "grande spazio", dal "grande spazio" si è condotti ad una nozione già più differenziata, a quella di reichischer Raum, o secondo il termine da noi stessi usato, di "spazio imperiale"» (Evola [1942] 558 col. II - 559 col. I). Sul contesto complessivo ora notazioni importanti in RuschI (2007).

2 Cfr. Joerges (2003).

3 Gli scritti di Carl Schmitt sul nostro tema sono stati quasi tutti meritoriamente raccolti nel volume Sснмiтt (1995). Per una prima inquadratura della questione vedi Blindow (1999). Per una utile sintesi delle principali teorie geopolitiche cfr. almeno JeAN (2010) 26 ss. Dal punto di vista giuridico resta decisivo Losano (2005).

4 Losano (2006). 
che ora culmina in uno dei più approfonditi e compiuti panorami disponibili sul tema. ${ }^{5}$

Losano non è l'unico a notare un elemento chiave, che il preambolo e l'incipit di tale bozza di trattato (23 febbraio 1943) restituisce con tutta evidenza.

Il significativo testo dell'art. 1 (dei 9 più una considerazione conclusiva) appunto recita:

«Scopo della politica dei Grandi Spazi è l'unione volontaria in Comunità degli Stati indipendenti dei Grandi Spazi. Le Comunità di Stati sono persone giuridiche sovrastatali, cui incombe il compito di assicurare la libertà ed indipendenza dei Grandi Spazi e dei loro Stati e Popoli, di proteggerli e difenderli da influenze e potenze intruse [ovvero più esattamente 'estranee allo spazio'; "raumfremde Mächte"], di rappresentare gli interessi della Comunità tanto verso l'interno quanto verso l'esterno, di bilanciare gli interessi di Stati e popoli dei Grandi Spazi secondo il principio della giustizia e dell'equità, ponendosi così al servizio del benessere comune di tutti quegli Stati e Popoli, nell'ambito politico, economico, culturale e sociale». ${ }^{6}$

I passi appena ascoltati vengono dunque letti da Losano secondo l'intenzione del preambolo, nel senso che essi facciano dunque emergere la «necessità storica di trasformare i Grandi Spazi in Comunità internazionali di tipo nuovo e con una propria personalità giuridica»; e, conseguentemente, subito dopo può significativamente concludere:

«il Grande Spazio, inteso come insieme di uno Stato-guida e più Stati a sovranità limitata [su questa interpretazione, almeno in relazione al concetto schmittiano, dovremo però rapidamente tornare], sarebbe dovuto divenire un soggetto con una sua personalità giuridica. Alla distinzione classica fra Stato nazionale e comunità internazionale di Stati paritetici si aggiungeva cioè un terzo elemento: la Comunità di Stati, meno unitaria dello Stato nazionale, ma più coesa e gerarchica della comunità internazionale. Di conseguenza, accanto alla dicotomia tradizionale fra diritto nazionale e diritto internazionale, avrebbe dovuto porsi come terza categoria un non meglio precisato "diritto comunitario"»."

5 Losano (2011).

6 Losano (2006) 250-252; alle pp. 250- 256 è editato per la prima volta il testo tedesco del progetto di trattato ultimato il 23 febbraio 1943 dal Vortragender Legationsrat Kolb (Vertrag zwischen Deutschland, Italien und Japan über die politische Gestaltung der Großräume Europa und Großostasien, in: Politisches Archiv des Auswärtigen Amts, Nachlass Cécil von Renthe-Fink, $B d$. 12) accompagnato dalla traduzione italiana. L'analisi sull'argomento è poi ripresa anche in Losano (2011) 67-73.

7 Losano (2006) 237-256, 247. 
Lo stesso analista del testo 'riscoperto', allora - ma con lui altri (per esempio, appunto, Proelss, Mathias Schmoeckel, o il già citato Christian Joerges, con Navraj Singh Ghaleigh, che parlano, sempre aderendo al coro 'polemico', di "Darker Legacy of Law in Europe") -, non può trattenersi dal soggiungere: «Oggi quest'ultimo termine indica il diritto dell'Unione Europea. Indubbiamente v'è qualche assonanza fra la concezione dittatoriale dei Grandi Spazi e le concezioni federalistiche del dopoguerra, poiché l'una e le altre fanno riferimento a strutture sopranazionali». 8

Ma, posta l'interpretazione del legame biunivoco fra Grandi Spazi e dittature - i Grandi Spazi sono "delle dittature" -, e, d'altro canto il favore per l'europeismo (come potrebbe avere, in compagnia dei padri nobili da De Gasperi a Schumann a Spinelli - ma ricordiamo anche Adenauer -, ascendenze culturali di tal tipo?), ecco che il filo 'deve' immediatamente interrompersi: «[...] ma le somiglianze finiscono qui. Le dissonanze sono invece ben maggiori, e riguardano i punti centrali dei due tipi di unione: il Grande Spazio viene imposto attraverso l'espansione bellica, mentre la comunità postbellica si forma attraverso trattati internazionali».

Così a sostenere che il filo, esile quanto si voglia - ma lo stesso Schmitt nel 1978, con riferimento e quasi 'per bocca' del suo allievo Hans Peter Ipsen, autore nel 1972 di un imponente Europäischen Gemeinschaftsrecht, avrebbe piuttosto parlato di "tiefen Trauer" per una occasione perduta, visto che l'intensità politica dell'Europa restava ai suoi occhi evidentemente di gran lunga lontana dall'intensità della decisione politica globale contraria: «Die weltpolitischen Kräfte und Mächte, die um die politiche Einheit der Welt kämpfen, sind stärker als das europäische Interesse an der politischen Einheit Europas» ${ }^{9}$-; così a sostenere che il filo di continuità sussiste, dicevamo, possono continuare ad essere solo coloro che dichiaratamente siano avversari dell'unificazione europea: 'solo' ( $\mathrm{ma}$ in realtà il dibattito trascende di gran lunga questo tipo di 'segmentazione') costoro - è paradigmatico in proposito il lavoro di John Laughland ${ }^{\mathbf{1 0}}$ - hanno l'interesse a presentare «quest'ultima come la tardiva e indiretta realizzazione del progetto di Grande Spazio».

8 Losano (2006) 247. Certamente, ad avviso di Losano, «le somiglianze finiscono qui».

9 Vedi Mehring (2008) 114.

10 Laughland (1998), sul quale vedi anche Proelss (2003). 
Se, come è stato affermato in occasione della nascita della prima rivista italiana espressamente dedicata alla geopolitica nel secondo dopoguerra, "Limes" (1993), la geopolitica può aspirare ad essere un valido ausilio alla comprensione del mondo odierno, ciò sarà a patto che si sgombri il campo da un «primo equivoco: più che una scienza, la geopolitica è un sapere nel senso di Foucault, o meglio un ragionamento"; se, ancor di più, va sottolineato che «non esiste produzione geopolitica slegata da un interesse politico. Per definizione, ogni geopolitica è operazione ideologica diretta a soddisfare gli interessi politici di un gruppo definito territorialmente» (Antonsich); se quindi, in una prospettiva luhmanniana, i concetti che ne derivano (incluso il Raumbegriff) non andrebbero considerati essenzialisticamente, ma come strumenti operazionali, come forme operazionali politico-giuridiche che non escludono affatto la coesistenza di altre forme di 'codificazione' del potere a carattere non spaziale o a rete (J. Kleinschmidt): se tutto questo è plausibile, allora si comprende come la riflessione giuridico/geopolitica acquisisca proprio oggi, nel XXI secolo - al di là delle sue ambigue origini - tutta la sua pregnanza. ${ }^{\mathbf{1 1}}$

E questo anche (e talvolta, appunto, si ha la sensazione che ciò avvenga soprattutto laddove più a cuore stanno globalizzazione e deterritorializzazione) nei campi più inattesi.

Visto che - in modo del tutto paradossale, in realtà, perlomeno sotto il profilo che qui interessa - nel mondo anglosassone, e statunitense in particolare, lo 'sdoganamento', per non dire la 'renaissance', del giurista di Plettenberg è, a detta di molti, da far risalire al "unilateral turn” dell'amministrazione guidata dai c. d. neoconservatori, non sarà inutile soffermare per un momento la nostra attenzione proprio su di un documento operativo, da quella proveniente e non molto noto, per scorgere come il vocabolario e la grammatica del 'sapere negato' che stiamo indagando, innervi ben in profondità il discorso 'riservato'.

Ci riferiamo ad un promemoria di Donald Rumsfeld del 2001 intitolato "Punti da discutere: voci del bilancio FY01 e FY02-07", ma che in gran parte non trattava aspetti di bilancio tradizionale, quanto un quadro di prospettive politiche internazionali, con ricadute di aumento drastico del bilancio militare, già nella roadmap verso la guerra irachena. 
«1. Il crollo dell'Unione Sovietica ha generato forze centrifughe che hanno creato nuove potenze regionali nel mondo. Alcune di queste sono fortemente ostili agli Stati Uniti e si stanno armando per impedirci di far pesare il nostro potere convenzionale o nucleare in una crisi regionale.

2. La liberalizzazione successiva alla Guerra fredda del commercio, di beni e servizi tecnologici ha consentito alle nazioni più povere della terra di acquistare rapidamente la più distruttiva tecnologia militare mai ideata, comprese le armi nucleari, chimiche e biologiche e i loro mezzi di impiego. Non è possibile impedirlo.

3. Attualmente il settore civile, non quello della difesa, crea le tecnologie che permettono di accrescere le capacità militari. Tali tecnologie disponibili a tutti possono essere usate per creare risposte "asimmetriche" al nostro potere militare convenzionale da parte di Stati piccoli o medi che non possono sconfiggere le nostre forze, ma possono impedire l'accesso ad aree critiche in Europa, nel Medio Oriente e in Asia.

I sommergibili convenzionali, la difesa aerea avanzata, gli attacchi alla nostra infrastruttura C4ISR [Comando, Controllo, Comunicazione, Computer, Informazioni, Sorveglianza e Ricognizione] e approcci "asimmetrici" di questo tipo possono limitare la nostra capacità di usare la potenza militare.

4. La Cina, la Russia, l'Iran, l'Iraq, la Corea del Nord e altri paesi stanno investendo in queste risorse che sfruttano importanti lacune nelle capacità degli Stati Uniti. Il commercio internazionale liberalizzato estenderà queste risorse ad altri.

5. Le minacce possono emergere molto rapidamente e con poco o nessun preavviso.

NOTA. Osservazione dell'ex segretario alla Difesa Bill Cohen: la minaccia missilistica contro gli Stati Uniti potrebbe emergere entro un anno.

CONCLUSIONE. Il rischio per la sicurezza degli Stati Uniti e dell'Alleanza cresce nella misura in cui gli Stati Uniti non rispondono con efficienza e decisione alle minacce asimmetriche che caratterizzeranno probabilmente il primo quarto del XXI secolo». ${ }^{\mathbf{1 2}}$

Come ben si vede - e sarà esplicitato nella nozione di preemptive strikes (peraltro, cosa poco sottolineata, già isolatamente teorizzata da un 'irregolare' della politica e della strategia militare europea, Federico il Grande, ironicamente nel suo 'Antimachiavelli') - la distinzione classica fra Stato nazionale e comunità internazionale di Stati paritetici, che caratterizza la

12 Vedilo nella testimonianza dell'allora Segretario del tesoro O’Neill in Suskind (2004) $88-89$. 
visione 'a-spaziale' (interstatale) propria della concezione tradizionale del diritto internazionale (lo Jus publicum europaeum), e il suo concetto di sovranità dello Stato, che impedisce l'intervento nei suoi 'affari interni', e, appunto, la conseguente tendenziale uguale legittimazione degli stessi, che culmina nell'astratto jus ad bellum di ciascuno, sono ormai molto lontani dall'orizzonte.

L'equilibrio e la limitazione (della guerra in forma) erano i concetti costituzionali di quell'ordine internazionale, così come qualche autore già nel XIX secolo intravedeva: «Nel diritto internazionale del XIX secolo venne anche spesso propugnata la dottrina secondo cui l'equilibrio degli stati, se non la vera base, è tuttavia una eventuale garanzia accessoria del diritto delle genti». ${ }^{13}$

Essi sono ora sostituiti appunto da un 'dimensionamento asimmetrico' degli Stati, che se non usa in modo giuridico-formale l'espressione «insieme di uno Stato-guida e di più Stati a sovranità limitata», ne rispecchia abbastanza l'intenzione 'concreta', opponendo all'asimmetria dissolvente un radicale recupero del concetto di egemonia, a quello di Grande Spazio spesso connesso.

Anche se si tratta ormai di una egemonia su di uno spazio indifferenziato, uno «spazio 'liscio', disorientato» (C. Galli), appunto perché privo di ordine: come emerge paradossalmente anche dal promemoria di bilancio appena richiamato, «il libero commercio [...] non mette in crisi soltanto il diritto pubblico tradizionale strettamente collegato all'idea di Stato nazionale effettivamente sovrano, ma lo stesso diritto internazionale, reso spettrale dalla mancanza di spazi e luoghi propri $[\ldots] »$ (M. Cacciari). ${ }^{\mathbf{1 4}}$

Insomma, come aveva avuto modo di puntualizzare il nostro ancora nel 1951, «l'ideale dell'unità globale del mondo in un perfetto funzionamento

13 Il saggio di Schmitt del 1939 (Völkerrechtliche Großraumordnung mit Interventionsverbot für raumfremde Mächte. Ein Beitrag zum Reichsbegriff im Völkerrecht; vedi sotto nota 20) era stato tradotto in italiano nel 1941, con diverso ma significativo titolo, da Francesco Pierandrei; ora tale traduzione è di nuovo accessibile, con il titolo scelto dal traduttore italiano, Sснмітт (1996). Per la frase citata vedi p. 6 e nota 4. Sulla centralità necessaria dell'equilibrio per il diritto internazionale interstatale, e quindi della necessaria presenza, quali 'custodi' e 'veri garanti' del diritto internazionale delle potenze neutrali, cfr. anche Schmitt (2007a) 513.

14 Citato nell'interessante saggio di Feliziani (2007-2008) 161-177. 
ha relazione con l'attuale pensiero tecnico-industriale»; esso, naturalmente, non poteva essere confuso con quello cristiano, posto che «lo sviluppo tecnico produce necessariamente organizzazioni e centralizzazioni sempre maggiori, e si potrebbe dire che oggi il destino del mondo è la tecnica, più che la politica, la tecnica come processo irresistibile di centralizzazione assoluta». ${ }^{\mathbf{1 5}}$

Non per caso tale riflessione, dominata dalla visione tecnico-industrialfinanziaria dell'unità del mondo, lascia appigli testuali per poter essere considerata una estensione modernizzatrice, da un lato della dottrina della «sicurezza delle vie di traffico dell'Impero britannico» - di cui una più definita espressione era costituita dalla "dottrina di Disraeli" del necessario appoggio invariato alla Turchia come questione vitale per l'Impero britannico - e, dall'altro, di quanto lucidamente nel 1932, veniva proclamando la c. d. "dottrina Stimson", dal nome dell'allora ministro degli Affari Esteri americano Henry L. Stimson. ${ }^{16}$

Una tesi paninterventista che venne poi, in una conferenza del 9 giugno del 1941, più compiutamente precisata dal suo stesso autore, «usando parole che costituiscono un vero credo della nuova unità del mondo», ed affermando senza mezzi termini che «la terra, oggi, non è più grande degli Stati Uniti nel 1861, già troppo piccoli per l'antagonismo tra Stati del Nord e Stati del Sud: la terra è troppo piccola [...] per due sistemi contrapposti». ${ }^{\mathbf{1 7}}$

Non sorprenderà allora vedere di recente autori statunitensi o comunque di area anglosassone - anche a prescindere dalla notazione che solo nel 2003 Der Nomos der Erde è stato reso accessibile in traduzione inglese per merito di

15 Sснмiтt (1994a) 307-309.

16 Inizialmente, e da qui ovviamente l'interesse di Schmitt, tale impostazione concerneva la dottrina Monroe asiatica o giapponese, c. d. Asia "Monroe-Shugi". Infatti «La nota del segretario di stato americano Stimson ai governi di Cina e Giappone del 7 gennaio 1932 contiene la dichiarazione che il governo degli Stati Uniti non ammetterà né la legalità di qualsiasi situazione de facto né riconoscerà trattati stipulati fra Giappone e Cina, che violino i diritti contrattuali degli Stati Uniti o dei suoi cittadini in Cina, incluso quei trattati che si riferiscono alla sovranità, all'indipendenza $\mathrm{o}$ all'inviolabilità territoriale $\mathrm{e}$ amministrativa della repubblica cinese o a quella politica internazionale riguardo la Cina che è nota come la 'politica della porta aperta'. Una risoluzione dell'assemblea della Società Ginevrina delle Nazioni dell'11 marzo del 1932 formula in modo corrispondente il principio del non riconoscimento di tutte le situazioni o trattati, che sono stati realizzati in contrasto con il patto della Società Ginevrina delle Nazioni o con il patto Kellogg».

17 Schmitt (1994a) 306. 
G. L. Ulmen (The Nomos of the Earth), posto che nella prospettiva dell'interesse internazionalistico di Schmitt esso occupa in realtà piuttosto il ruolo di una summa certo teoreticamente impegnativa, ma pure per più versi 'mitizzante' - cogliere un'attualità di prospettive su tematiche specifiche, come l'utilizzazione della analisi relativa al diritto di occupazione sviluppata nel 1925 ("La Renania come oggetto di politica internazionale" - Die Rheinlande als Objekt internationaler Politik e cfr. dello stesso anno Der Status quo und der Friede) in riferimento all'Iraq War, oppure del saggio del 1963 in relazione all'esclusione post 9/11 dei terroristi dalla categoria "legal combatans" della Convenzione di Ginevra e quindi della "Road to Abu Ghraib".

Che la dottrina internazionalistica schmittiana, ovvero la parte per solito trascurata quando non, come detto, ritenuta la più compromessa, e quindi inutilizzabile, della sua riflessione, trovi attualmente tale risonanza nel mondo anglosassone - gli esempi si potrebbero moltiplicare - è allora forse un segnale che, come si accennava, non va sottovalutato, né affrettatamente attribuito, e per più ragioni.

Anzitutto emerge una chiara indicazione, per così dire, di carattere 'storiografico', ossia la centralità che a tale riflessione va attribuita per l'interpretazione complessiva, posto che implica il riconoscimento anche della sua 'originarietà' e continuità nel tempo.

Non si tratta dunque affatto di un interesse tardivo, come vuole, ad esempio, un seguace dell'importanza di Lucien Freund, secondo il quale il suo maestro si sarebbe orientato verso il diritto internazionale soltanto dal 1936, a seguito delle minacce rivoltegli dall'ala più intransigente e fanatica del partito nazista, «essendo ormai troppo pericoloso continuare ad occuparsi di questioni di politica interna», ma invece risalente ai primi anni venti (S. Carloni) e continuativo nel tempo (basterà ricordare Die Kernfrage des Völkerbundes, 1926; Der Völkerbund und Europa, 1928; Völkerrechtliche Formen des modernen Imperialismus, 1932; Völkerrechtliche Großraumordnung mit Interventionsverbot für raumfremde Mächte. Ein Beitrag zum Reichsbegriff im Völkerrecht, 1939; la serie di articoli pubblicati fra il 1941 e il 1943: Staatliche Souveränität und freies Meer. Über den Gegensatz von Land und See im Völkerrecht der Neuzeit; La mer contre la terre; Raumrevolution. Vom Geist des Abendlandes, Behemoth, Leviathan und Greif. Vom Wandel der Herrschaftsformen, la monografia del 1942 Land und Meer. Eine weltgeschichtliche Betrachtung, i saggi del secondo dopoguerra, poi raccolti in varia forma, fino a giungere tra gli ultimi, ma non certo per importanza, al 1978 de "La rivoluzione legale 
mondiale. Plusvalore politico come beneficio per la legalità giuridica e la superlegalità").

In secondo luogo, una ancora più chiara indicazione sul difficile tema della 'compromissione' e contestualizzazione.

È certo innegabile una connessione 'occasionalistica' (profondamente radicata, se accettiamo l'analisi di K. Löwith, in tutto l'habitus spirituale e culturale del nostro) o, se si preferisce, opportunistica, di molti di questi lavori con concreti aspetti della politica ed anche propaganda di regime.

Il quadro che ne offre Carloni può a questo proposito essere considerato esaustivo: «Questo stupefacente tempismo occasionalistico (il quale altro non è se non una manifestazione del suo intrinseco nazionalismo) spiega sia il grande risalto acquisito presso la stampa interna ed internazionale nella quale egli è presentato come il teorico della politica espansionista del Terzo Reich - sia il richiamo che lo stesso Hitler fece ad una "dottrina Monroe europea"; un accenno a cui Schmitt si adeguò prontamente superando il concetto di großdeutsches Reich in quello di un sgrande spazio europeo> (europäischer Großraum). E spiega anche il giudizio fortemente negativo paradigmaticamente fissato, tre anni dopo, da Franz Neumann allorché indica in Schmitt la voce predominante del coro "revisionista" nazionalsocialista e uno dei principali ideologi dell'espansionismo hitleriano. Nel frattempo il regime aveva imparato a sufficienza la lezione del suo Kronjurist: nel rispondere, il $1^{\circ}$ luglio 1940, all'ammonimento del segretario di Stato Hull, secondo il quale gli Stati Uniti non avrebbero potuto assecondare «ogni tentativo di trasferire una regione geografica dell'emisfero occidentale da una potenza non americana a un'altra potenza non americana), Joachim von Ribbentrop concludeva minacciosamente come segue: $>$ Il governo del Reich coglie questa occasione per sottolineare che, in linea di principio, la non interferenza degli stati europei negli affari del continente americano non può essere giustificata a meno che gli stati americani, da parte loro, si astengano parimenti dall'interferire negli affari del continente europeo<. ${ }^{\mathbf{1 8}}$

18 In Carloni (2009) 11-12. Il quotidiano inglese «Daily Mail» scriveva il 5 aprile di quell'anno: «Il signor Hitler e il professor Carl Schmitt si stanno ora adoperando per completare il quadro di questa concezione, e il Führer la presenterà quanto prima al mondo proprio per giustificare la spietata espansione della Germania», definendo Schmitt «l'uomo chiave della linea politica del signor Hitler». 
$\mathrm{Ma}$ anche volendo seguire punto per punto tale ricostruzione 'per accostamento', senza soffermarci partitamente sui suoi aspetti unilaterali, l'attuale recupero, sia pure fortemente dialettico, mostra che l'aspetto teorico e critico della dottrina dei 'Grandi Spazi' non può essere 'ridotto' al suo contesto politico originario.

Né si dica, replicando la stessa struttura di argomentazione, che esso possa essere 'ridotto' all'attuale riemergere di orientamenti 'neoconservatori', perchè altrimenti non si spiegherebbe che a tale dibattito partecipino anche esponenti di spicco della geopolitica critica e dei subaltern studies. ${ }^{\mathbf{1 9}}$

Ecco che allora, in terzo luogo, siamo stimolati a cercare una risposta più complessa alla domanda di questa rinnovata attualità, che, lo si noti, oltre alla centrale trattazione monografica del 1939 Völkerrechtliche Großraumordnung mit Interventionsverbot für raumfremde Mächte. Ein Beitrag zum Reichsbegriff im Völkerrecht (Ordinamento giuridico internazionale dei grandi spazi con divieto d'intervento per le potenze estranee allo spazio. Un contributo al concetto d'impero nel diritto internazionale), comprende fondamentalmente i saggi poi raccolti nel 1940 sotto il significativo titolo di Positionen und Begriffe im Kampf mit Weimar - Genf - Versailles 1923-1939, Hanseatische Verlagsanstalt, Hamburg-Wandsbek 1940 (Posizioni e concetti in lotta con Weimar - Ginevra - Versailles 1923-1939).

Essa passa inevitabilmente per la stessa operazione a cui, in particolare nel 1939, costruendo la sua posizione, Schmitt ha sottoposto la 'dottrina Monroe' - costruita in funzione difensiva contro l'interventismo della Santa Alleanza in Sudamerica e sintetizzata comunemente dalla massima «l'America agli Americani» - dalla quale ha preso, com'è noto, le mosse: «La vera, originaria dottrina Monroe, qual è scaturita dallo spirito di John Quincey Adams e come è stata pronunciata nel messaggio del presidente Monroe del 2 dicembre 1823, contiene tre semplici idee: indipendenza degli Stati americani; non colonizzazione in questo spazio; non intromissione di potenze extraamericane in questo spazio, unitamente alla non intromissione dell'America nello spazio extraamericano». ${ }^{20}$

A Schmitt non sfugge che il ruolo fondamentale poi assunto nella politica estera degli Stati Uniti ha fatto sì che essa subisse una radicale torsione, e che,

19 Vedi per un solo esempio dei molti che si potrebbero addurre Mouffe (2005).

20 Sснмiтt (2007c) 491. 
nel corso dell'Ottocento, da un principio di duplice non-intervento, si trasformasse in argomento legittimante una politica espansionistica nell'area latinoamericana - dall'appoggio al Venezuela nella controversia con la Gran Bretagna del 1895 fino agli interventi a Cuba, Haiti, Santo Domingo, Panama e nel Nicaragua -, in modo tale che «questo pronunciamento difensivo di un piccolo Stato coloniale [...] è divenuto uno strumento di diritto internazionale dell'egemonia di questo Stato sul grande continente americano» (1932). ${ }^{21}$

Ma egli adesso lavora da giurista e non soltanto come storico delle forme internazionalistiche di imperialismo - quando ne aveva sottolineato "la stupefacente elasticità ed estensibilità", ricordando come «questa apertura davanti a tutte le cose, anche all'alternativa diritto o politica, è a mio parere tipico di ogni autentico e grande imperialismo. Non è pensabile che una grande potenza e ancor meno una potenza mondiale imperialista si attenga stabilmente dal punto di vista giuridico ad un codice di concetti e norme fisse, che uno straniero posto all'esterno possa applicare contro essa stessa. L'essenziale lo ha così riassunto Hughes nel 1923: la definizione, interpretazione e applicazione della dottrina Monroe è cosa degli Stati Uniti» (1932). ${ }^{22}$

E se è così, se adesso è il giurista a prendere soprattutto la parola, allora «le singolarità di questo sviluppo qui non interessano».

Infatti l'operazione è anche per parte sua costruttiva: è essenziale [ma appunto al nuovo specifico fine giuridico-concettuale] che la dottrina di Monroe resti autentica e non falsificata, fintantochè è fissa «l'idea di un grande spazio concretamente determinato, nel quale potenze estranee allo spazio non possono immischiarsi.».

Si trattava perciò, in ultima analisi, di ricavare da essa il nucleo concettuale giuridicamente utilizzabile, perché nell'oscillazione fra diritto e politica il pendolo deve puntare su 'diritto', perché da una dottrina della cui natura si dubitava deve scaturire un principio giuridico di (un nuovo) diritto internazionale: «Noi quindi non imitiamo semplicemente un modello americano, quando ci riferiamo alla dottrina Monroe; noi liberiamo soltanto il nocciolo 
sano di un principio giusinternazionalistico del grande spazio e lo sviluppiamo sensatamente per il nostro grande spazio europeo». ${ }^{23}$

Proviamo quindi anche noi a sottoporre a quell'operazione di 'estrazione' la dottrina schmittiana stessa, estrapolandone per punti-chiave gli elementi a nostro avviso qualificanti:

a) anzitutto la dottrina dei Großräume appartiene a buon diritto alle teorie ordinamentali.

Essa prende dunque le mosse da una diagnosi sul progressivo esaurimento del diritto internazionale westfaliano, poi 'ginevrino' e infine post-UNO e va alla ricerca di un nuovo principio ordinatore, tenendo conto che le grandi fasi della storia del diritto internazionale sono sempre segnate da una 'rivoluzione spaziale': «Mentre l'idea dello spazio contiene un punto di vista della delimitazione e della divisione e per questo enuncia un principio giuridico ordinatore, la pretesa universalistica di intromissione mondiale distrugge ogni delimitazione e distinzione razionale». ${ }^{24}$

b) la scelta giuridica per il concetto e quindi per il limite distintivo ci fa capire inoltre che ci troviamo di fronte ad una visione dell'ordine internazionale di carattere pluralistico e multipolare.

La presa di distanza nei confronti dell'universalismo non va equivocata come avversione all'idea di unità, ma deve essere semplicemente intesa, come ha ben visto Koskenniemi, come critica nei confronti di quello che viene inteso come un falso universalismo cosmopolitico. Quando ancora siamo nel ormai lontano 1951 - non si era verificata la fine della 'guerra fredda', ma essa già appariva nel suo orizzonte concettuale, la prospettazione di tali possibilità pluralistiche era già lucidamente presa in considerazione: «Se l'unità è in sé cosa buona, la dualità è in sé cattiva e pericolosa. "Binarius numerus infamis", dice San Tommaso [...] Per la tendenza generale verso l'unità tecnico-industriale del mondo, la dualità attuale non può essere altro che la transizione verso l'unità, la fase ultima, l'ultimo "round" della lotta per l'unità definitiva [...] È indubitabile che oggi esistano marcate tendenze a formare una cosiddetta "terza forza". Non vado a trattarne le diverse possibilità immaginabili. Ciò darebbe vita ad una discussione politica particolarmente interessante ed attuale ma che voglio evitare; per esempio sulla situa- 
zione e l'importanza come possibile terza forza della Cina, dell'India, dell'Europa, del Commonwealth, del mondo ispanico, del blocco arabo, o di altre formazioni imprevedibili, che costituirebbero una pluralità di grandi spazi, e forse un nuovo equilibrio $[\ldots] \gg .{ }^{25}$

c) in terzo luogo, si tratta di una dottrina non sic et simpliciter antistatuale, come pure è stato sostenuto, ma piuttosto post-statuale.

Essa - al contrario del kelsenismo (o di impostazioni analoghe, alla Krabbe, per intendersi), al contrario dei sostenitori del 'diritto sconfinato', della concezione, in realtà a-spaziale, di uno 'spazio giuridico globale' che si identifica con la rete delocalizzata delle moderne tecnologie e della finanza mondiale, ma al contrario infine anche delle versioni apparentemente 'speculari' (alla Negri-Hardt) - non rinuncia al concetto di sovranità, ma tende a 'dislocarlo'. Qui si aprirebbe la specifica questione, cui possiamo solo accennare, e che Schmitt definisce "basilare" nel 1939, ma è sottoposta ad evoluzione nella riflessione successiva, dell'«interdipendenza delle idee di impero, "grande spazio" e principio di non intervento». ${ }^{26}$ Resta fin dal principio comunque inteso che "naturalmente "grande spazio" non equivale a impero nel senso che l'impero si identifichi col "grande spazio" da esso sottratto agli interventi altrui; né ogni stato od ogni popolo all'interno del "grande spazio" forma per sé medesimo una frazione dell'impero». Per molti versi «il concetto di stato come concetto centrale del diritto delle genti è sorpassato poiché non corrisponde più alla realtà efficiente e molti se ne sono già resi conto», soprattutto però nel senso che vada eliminato un effetto del formalismo statualistico, cioè «l'altro errore, parimenti pericoloso per il diritto internazionale, che consiste nell'isolare di fatto $i$ singoli enti politici sopprimendo ogni loro interdipendenza».

d) in quarto luogo, siamo di fronte alla proposta di una teoria dell'equilibrio.

Certo ci muoviamo ormai su di un piano molto lontano, già solo per quanto accennato in precedenza, dal discorso della bilancia o del 'concerto europeo', anche se lo diremmo 'analogico' rispetto a quello. ${ }^{27}$

25 Sсhмitт (1994a) 307-309.

26 Sснмiтt (2009) 64 ss.

27 Interessante è il legame che Schmitt, ad un certo punto, istituisce (anche) tra questa dottrina e il tema del katechon; cfr. Sснмiтt (1994c) 261-269, spec. 268-269. Su questa prospettiva, che non possiamo qui ulteriormente approfondire, vedi ora CACCIARI (2013). 
Si tratta appunto del tentativo di trasposizione alla nuova fase della dottrina secondo cui l'equilibrio delle potenze o degli stati era una eventuale garanzia accessoria del diritto delle genti, quando non addirittura la sua vera base. Una posizione che, di nuovo, va al nocciolo concettuale dell'idea, se presa alla lettera altrimenti troppo poco realisticamente plausibile o suscettibile di sviluppo, di 'terza forza'.

In una sintesi che assurge, per la sua icasticità, contemporaneamente a ricapitolazione 'decisiva' e compito per la riflessione internazionalistica che verrà, si coglie la radice profonda di tutto il suo percorso: «Per questo la possibilità di una terza forza non suppone il limite numerico di tre: può svilupparsi in una pluralità. Ciò implica la possibilità di un equilibrio delle forze, un equilibrio di vari grandi spazi, che creino tra loro un nuovo diritto delle genti, ad un nuovo livello, e con dimensioni nuove, però, nello stesso tempo, dotato di certe analogie con il diritto delle genti europee dei secoli XVIII e XIX, che pure si basava su di un equilibrio di potenze grazie al quale si conservava la sua struttura. Anche lo jus publicum europaeum implicava una unità del mondo. Era una unità eurocentrica: non era il potere politico di un unico padrone di questo mondo, ma di una formazione pluralista e di un equilibrio di varie forze. I numeri dispari (tre, cinque, ecc.) sono qui preferiti rispetto ai pari, perché rendono meglio possibile l'equilibrio. E' molto probabile che l'attuale dualità del mondo sia più vicina ad una pluralità che all'unità definitiva, e che siano troppo affrettati i pronostici e le combinazioni del One world». ${ }^{28}$

e) in ultima analisi, questa dottrina 'costruttiva' in vista di un nuovo Pluriversum, di un nuovo diritto delle genti, tale si manifesta in quanto contemporaneamente si ponga, rispetto "ad un sistema generale di intromis-

28 Sснмiтt (1994a) 309. Una notizia di qualche tempo fa, sfuggita ai più, sembra appunto testimoniare che il 'superamento' del pluriversum in un unico spazio globale è in realtà una 'descrizione pro futuro' di un dover essere (politicamente magari fortemente voluto $\mathrm{da}$ alcuni) più che una mera diagnosi (Avvenire, martedì 23 agosto 2011, 23): Bangladesh. Dopo cinquant'anni tracciato il confine con lo Stato indiano: «Dopo oltre mezzo secolo India e Bangladesh hanno tracciato il loro confine di 4.156 chilometri risolvendo una lunga disputa territoriale. I rappresentanti dei due governi hanno messo a punto la scorsa settimana un accordo che include 1.149 mappe di 5 porzioni della lunga frontiera che taglia in due il vasto delta del Gange e dove avvengono spesso scontri fra gli eserciti. L'intesa sarà siglata dal premier Manmohan Singh nella sua visita agli inizi di settembre. L'“evento storico" giunge dopo una serie di fallimenti a partire dal 1952 quando la regione faceva parte del Pakistan orientale e poi anche dopo l'indipendenza del Bangladesh del 1971. 
sione delocalizzata" generato dalla concezione di un "libero" commercio mondiale e del "libero" mercato mondiale, come teoria critica.

Adesso siamo forse meglio in grado di avvicinarci ad una spiegazione della rinnovata, seppur discussa, 'fortuna' di un simile itinerario. La categoria del Großraumordung, intesa nel suo 'nocciolo sano', come dottrina critica, ordinante, post-statuale e pluralista, fondata sui principi dell'equilibrio e del non intervento potrebbe trovare molteplici, 'dispari', interessati al suo approfondimento, ovvero tutti coloro che geopoliticamente non si riconoscono o vorrebbero non riconoscersi nella versione ancora dominante di 'comunità internazionale di (soli) Stati' o nell'idea di Impero americano o tanto meno in quella di Impero globale (Hardt / Negri e seguaci): dall'India alla Cina, dal Brasile all'America latina nel suo complesso, alla Russia, forse ad una certa idea dell'Europa o magari dell'Africa.

Ma parlavamo appunto di avvicinamento. Vi è forse infatti un altro aspetto, più interno questa volta alla configurazione tecnico-giuridica, sia pure sempre a livello di principi fondamentali, del diritto internazionale che seppur sinora ci paia non individuato in modo esplicito da alcuno tra coloro che hanno intrapreso questo dibattito, sta a nostro avviso al fondo del problema.

Vogliamo in conclusione cercare di esplicitarlo ridando voce ad un compianto collega e amico che, del tutto indipendentemente, ed anzi in lontananza, dalla riflessione schmittiana sulla quale ci stiamo affaticando, lo aveva colto ed espresso da par suo in una opera rimasta troppo 'segreta', e che andrebbe invece, specialmente oggi, riportata al centro del dibattito internazionalistico: alludiamo al volume Il diritto internazionale come sistema di diritto comune (1999) di Giovanni Battaglini.

In sintesi egli individua un effetto storico paradossale negli svolgimenti che dall'Atto di Helsinki del 1975 - che «ha allargato alle relazioni internazionali in generale degli Stati partecipanti il divieto della minaccia o dell'uso della forza, non soltanto "contro l'integrità territoriale o l'indipendenza politica di qualsiasi Stato", ma in qualunque altra maniera - ancorché si tratti di rappresaglia - incompatibile con i fini delle Nazioni Unite, per i quali si è additato dalla loro "Carta" il Consiglio di Sicurezza come l'organo titolare del monopolio funzionale dell'uso della forza per l'appunto in vista della "sicurezza" oltre che della pace mondiale» - portano all' ultimo decennio del XX secolo, e, a ragion veduta, anche oltre quella soglia fino ai giorni nostri. 
Effetto storico paradossale che deriva dal fatto dell'attestarsi delle N.U. «sulla prassi della "autorizzazione" esplicita (con apposita Risoluzione), o implicita (con silenzio-consenso) dello stesso Consiglio di Sicurezza agli Stati dal momento e per il fatto che non intraprende come e quando potrebbe "l'esercizio delle proprie funzioni a norma dell'art. 42" della Carta dell'O$\mathrm{NU}$; anzi rimuove, con ciò, senza lasciar dubbi, l'impedimento a che taluni Stati contraenti della Carta assumano direttamente l'uso della forza, in ispecie come ricorso estremo a una sanzione armata, pur sempre in nome della loro "unione" (ovvero quale "azione comune in nome dell'Organizzazione").» ${ }^{29}$

Si tratta di una vera e propria «involuzione delle Nazioni Unite in tema di pace e sicurezza internazionali», che si sostituisce alla ratio fondativa che le animava; tanto è vero che il Nostro parla addirittura di una «macchina del tempo in retrocessione» ${ }^{30}$ che va loro incontro.

Una retrocessione che, se non frenata, si incammina verso una totale involuzione e verso «l'egemonia, alla fine, di una sola grande potenza».

Ora lo stesso Battaglini, in un altro suo intervento, 'nomina', nel modo più sorprendente, questo processo di retrocessione: Dalle Nazioni Unite alla Società delle Nazioni. ${ }^{31}$

Se tale analisi coglie nel segno, e ci pare vi siano più indizi per poterlo ritenere, allora si comprende veramente appieno perché lo Schmitt di Positionen und Begriffe, il critico talora anche troppo ferocemente acuto del Doppio volto della Società ginevrina delle Nazioni (1926), il decostruttore dell'universalismo paninterventistico wilsoniano e non, il pensatore dei 'Grandi Spazi', possa di nuovo interrogarci, e magari offrire qualche elemento propositivo per individuare, per usare ancora di una sua espressione, un possibile kathecon che ci allontani dall'esito finale - purtroppo analogicamente ben noto - di quel processo involutivo.

Forse potremmo concludere il nostro breve itinerario di impostazione di una - ci pare a questo punto di poter dire - necessaria, più ampia ricerca, ricordando la parole che Vasco Pratolini consegnava alla rivista letteraria fiorentina di 'fronda' al fascismo, Campo di Marte (che infatti ebbe vita brevissima: dal 1 agosto 1938 al 1 agosto 1939): «Non di parole definite

29 Battaglini (1999) 221 ss.

30 Battaglini (1999) 222.

31 Battaglini (1992) 8 ss. 
noi abbiamo bisogno ma di idee importune che rendano perplessi e ci costringano ad una personale scoperta».

E certamente "l'ordinamento per grandi spazi", il Großraumordnung schmittiano, rispetto alle più correnti prospettive internazionalistiche proiettate verso l'unipolare' e il 'globale', è, perlomeno, proprio una di queste "idee importune".

\section{Bibliografia}

Battaglini, Giovanni (1992), Dalle Nazioni Unite alla Società delle Nazioni: un ritorno o una nostalgia?, in: Annali della Università di Ferrara, Nuova Serie, Sezione V, Scienze Giuridiche, vol. VI, 5-12

Battaglini, Giovanni (1999), Il diritto internazionale come sistema di diritto comune, Padova: Cedam

Buindow, Felix (1999), Carl Schmitts Reichsordnung. Strategie für einen europäischen Großraum, Berlin: Akademie Verlag

Cacciari, Massimo (2013), Il Potere che Frena. Saggio di Teologia Politica, Milano: Adelphi

Carloni, Stefano (2009), Dal Großraum al Nomos der Erde et retour: il pensiero internazionalistico di Carl Schmitt (available at: http://digilander.libero.it/ stefano.carloni/S_CARLONI-Il-pensiero-internazionalistico-di-Carl-Schmitt. pdf)

Evola, Julıus (1942), Spazi Vitali, Grandi Spazi e Spazi imperiali, in: Bibliografia fascista. Rassegna mensile a cura della Confederazione fascista dei Professionisti e degli Artisti, 585-593

Feliziani, Michele (2007-2008), La filosofia spaziale del pensiero politico di Carl Schmitt, in: Annali della Facoltà di Lettere e Filosofia dell'Università di Macerata XL-XLI, 161-177

Jean, Carlo (2010), Manuale di geopolitica, Roma, Bari: Editori Laterza

Joerges, Christian (2003), Darker Legacies of Law in Europe. The Shadow of National Socialism and Fascism over Europe and Its Legal Traditions, Oxford: Hart

Laughland, John (1998), The Tainted Source. The Undemocratic Origins of the European Idea, London: Warner Books

Losano, Mario G. (2005), La geopolitica nazionalsocialista e il diritto dei "grandi spazi", in: Materiali per una storia della cultura giuridica 1, 5-65

Losano, Mario G. (2006), Il mondo secondo Hitler. Un inedito progetto di trattato del 1943 tra Germania, Italia e Giappone, basato sulla teoria dei Grandi Spazi, in: Limes. Rivista italiana di Geopolitica 5, 237-256 
Losano, Mario G. (2011), La geopolitica del Novecento. Dai Grandi Spazi delle dittature alla decolonizzazione, Milano: Bruno Mondatori

Mehring, Reinhart (2008), "Raumrevolution” als Rechtsproblem. Zum politischen Kontext und Wandel von Carl Schmitts Großraumdenken, in: VoIgt, Rüdiger (Hg.), Großraum-Denken. Carl Schmitts Kategorie der Großraumordnung, Stuttgart: Franz Steiner Verlag, 99-117

Mouffe, Chantal (2005), Schmitt's vision of a multipolar world order, in: South Atlantic Quarterly 104, 2, 245-251 (available at: http://dx.doi.org/10.1215/ 00382876-104-2-245)

Proelss, Alexander (2003), Nationalsozialistische Baupläne für das europäische Haus? John Laughland's “The Tainted Source” vor dem Hintergrund der Großraumtheorie Carl Schmitts, in: forum historiae iuris (available at: www.for histiur.de/2003-05-proel/)

Ruschi, Filippo (2007), Leviatan e Behemoth. Modelli egemonici e spazi coloniali in Carl Schmitt, in: Jura Gentium. Rivista di filosofia del diritto internazionale e della politica globale III, 1-28 (available at: http:/www.juragentium.org/ topics/thil/it/ruschi.htm)

Suskind, Ron (2004), The Price of Loyalty. Georg Bush, the White House and the Education of Paul O’Neill, traduzione italiana, I segreti della Casa Bianca, Milano: Il Saggiatore

Schmitt, Carl (1994a), L'Unità del Mondo (1951), in: Schmitt, Carl (1994b), 303-319

Schmitt, Carl (1994b), L’Unità del Mondo e altri saggi. Introduzione e nota bibliografica di A. Campi, Roma: Antonio Pellicani Editore

Schmitt, Carl (1994c), La lotta per i grandi spazi e l'illusione americana (1942), in: Schmitt, Carl (1994b), 261-269

Schmitt, Carl (1995), Staat, Großraum, Nomos. Arbeiten aus den Jahren 1916-1969, Berlin: Duncker \& Humblot

Schmitt, Carl (1996), Il concetto di impero nel diritto internazionale. Introduzione di Pier Tommissen, Roma: Settimo Sigillo

Schmitt, Carl (2007a), Il Concetto di Reich (1939), in: Schmitt, Carl (2007b), $505-521$

Schmitt, Carl (2007b), Posizioni e concetti in lotta con Weimar - Ginevra - Versailles 1923-1939, a cura di Antonio Caracciolo, Milano: Giuffrè

Schmitt, Carl (2007c), Grande Spazio contro Universalismo (1939), in: Schmitt, Carl (2007b), 491-503

Schmitt, Carl (2007d), Forme internazionalistiche dell'imperialismo moderno (1932), in: Schmitt, Carl (2007b), 265-292

Schmitt, CARL (2009), Völkerrechtliche Großraumordnung mit Interventionsverbot für raumfremde Mächte. Ein Beitrag zum Reichsbegriff im Völkerrecht (1941), Berlin: Duncker \& Humblot (available at: http://dx.doi.org/10.3790/ 978-3-428-47110-2) 



\section{The Diffusion of Law Books in Early Modern Europe: A Methodological Approach}

\section{Introduction: Law Books across Temporal, Spatial and Disciplinary Dimensions}

Does the so-called "spatial turn" in the humanities and social sciences present new challenges or new perspectives for legal historians? Could a more precise awareness of the spatial and temporal dimensions reinforce their analytical skills?

One of legal history's traditional topics is the creative reading of texts in different contexts and periods - labelled as "reception", "transfer" or more recently as "translation" studies. ${ }^{1}$ The very identity of the legal historical discipline is strictly connected to the study of one of the most impressive cases of text "transmission" across two millennia, performed by the compilation later known as Corpus iuris civilis, ${ }^{2}$ and it abounds in examples of cross-bordering texts, schools and institutions. As a field of knowledge, the history of law could even be defined as a long history of interconnectedness between actors of different (sometimes even remote) spaces and times.

However, an attentive reflection on how these connections were made possible is still absent in legal history. ${ }^{3}$ In some cases, legal texts and schools were conceived from a strictly "national" perspective; in others, they seemed to have flowed freely through porous or inexistent frontiers. ${ }^{4}$ Both extremes

1 Duve (2012), 31-32.

2 The Byzantine codification of the Roman "heritage", which allowed its transmission over the centuries in the Western territories, has been suggestively defined as a "resistant container" as well as a "deforming mirror" (SChiavone [2005] 12).

3 I refer mainly to the cases of diffusion in the early modern period, for in the frame of the legal transfer studies important contributions have been issued. See for example Fögen/ Teubner (2005) 38-45 and the volume edited by Feest/ Nelken (2001).

4 The attempt to construct an essentialist view of the legal past of Europe as an idealized unity, expressed in works such as Reinhard Zimmermann's, has been extensively debated 
seem to be unrealistic. In an attempt to enhance our awareness of temporal and spatial dimensions, I will discuss some analytical tools to study processes of diffusion of printed texts which took place in a specific setting (continental Western Europe) and in a specific period (the early modern centuries).

I will begin with a few elementary questions: why were some legal works able to transcend their immediate local contexts while other similar works were not? Why, for example, did Salgado de Somoza's Labyrinthus creditorum or Juan Gutierrez's Consilia spread beyond the Pyrenees whereas the same did not happen to the writings of other Spanish and Portuguese jurists? An intuitive answer links, in our minds, the inherent "merit" of these works to their success among the readers of the past. We would be inclined to say that their works were somehow "better" than were those of their contemporaries on analogous topics. However, if we pick up an example taken from widely consumed didactical and practical works, such as the commentaries on the Digest or on the Institutes of Justinian, it is not easy to establish why such similar works deserved such dissimilar destinies. Why did certain commentators experience a wide European diffusion and others not?

I do not intend to deny that most of the popular works had indeed an "intrinsic merit". Neither do I aim to present an alternative view, irreconcilable with the parameters of the so-called "intellectual history", or even of the old history of ideas. ${ }^{5} \mathrm{I}$ do intend to present a complementary point of view, defying, though, the "merit" as the chief explanatory cause for the dissemination of some of the most influential legal works in the early modern centuries. Therefore, this reflection might affect the very formation of the "canon" in our discipline. Whether a highly "original" creation or a mediocre synthesis for law students, the popularity of a printed work in the early modern era depended on a complex set of reasons, where even chance and material conditions might have played a substantial role.

This approach benefits from a consolidated historical discipline, or "subdiscipline", known as "book history". Embracing its contributions - such as the reconsideration of notions such as text, author, reader, $\operatorname{transmission}^{6}-$

and criticized from different perspectives (Giaro [1995], Osler [1997], Cappellini/Sordi [2002], Birocchi, [2006]). The "European nature" of the discipline should be seen as a result of historical research rather than as a point of departure (SAVELLI [2011] XVI).

5 See the debates in: Collini (2014).

6 According to the widely repeated assertion of Chartier: «Se trata, por ende, de romper la actitud espontánea que nos hace suponer que todos los textos fueron compuestos o leídos 
could result in a rewriting of many chapters of European legal history. They seem to offer a fruitful path to enhance the awareness of the temporal and spatial dimensions in the legal historical investigation.

\section{Book History and Legal History: Points of Convergence}

During the early modern centuries, when the "state-centered" conception of political power developed alongside a fixed view of the space, ${ }^{7}$ the diffusion of legal texts occurred mainly (not exclusively ${ }^{\mathbf{8}}$ ) through the hand printing press. As printing shops rapidly spread throughout the European continent, the first printed editions of the Canon and the Civil law compilations were released together with the medieval authorities and new voices in the jurisprudence. The main schools of thought and cultural movements which influenced the legal culture during those centuries - such as Legal Humanism, the Usus Modernus Pandectarum or the Enlightenment, to quote only some examples - have spread thanks to the printed medium.

Although legal historians, with very few exceptions, do not usually consider their source (the printed book) as a material object belonging to a universe of practices (that of the printing culture of the early modern era), most of them would instinctively acknowledge a connection between the printing press and the diffusion of legal books in this period. In other words, it seems undeniable that printing technology enabled legal schools and authors «to transcend their immediate circumstances and communicate reliably with others in different times and places»; ${ }^{9}$ since books are precisely one of the ways of escaping the spatial and temporal constraints in the communication. ${ }^{10}$

en el pasado según las reglas o las prácticas que caracterizan la relación contemporánea con la cultura escrita» (CHARTIER [2000] 9). For a critique of the notion of "transmission" which is at the heart of the great bibliographic projects see Hellinga (1995) 64.

7 Hespanha (1993).

8 On the manuscript culture in the early modern centuries see, among others, BouzA Álvarez (2001).

9 Johns (1998) 40.

10 «Communiquer à distance, par l'écrit (manuscrit ou imprimé) ou par d'autres médias, c'est échapper à l'impératif de l'espace et du temps, permettre la mise en commun des savoirs en même temps qu'une réorganisation des modèles d'intelligibilité et des pratiques qui leur sont liés» (Barbier/Bertho Lavenir [2003] 7). 
This elementary assumption is not, however, as self-evident as it appears at first glance. To the common belief that printed word's importance «lie[s] precisely in its ability to transcend such local contexts and enable communication across wide distances», many historians today tend to consider «this power to transcend place as something itself in need of explanation». ${ }^{\mathbf{1 1}}$ Several investigations have put into question the features of universality, uniformity, fixity and reliability ascribed to printed texts in the early modern period. ${ }^{\mathbf{1 2}}$ The association between the printing press and the large-scale reproduction of uniform materials remains no longer undisputed.

In an effort to enhance the consciousness of the temporal and spatial dimensions in the study of widespread legal historical writings, two basic ideas might be especially enlightening: first, the "epistemic indeterminacy" of the printed texts in the age of the hand press; ${ }^{13}$ second, the focus on the reader. If the printed text was seen by its contemporaries as intrinsically untrustworthy, the task of the history of the book would consist of explaining how this indeterminacy «could be overcome to make knowledge and hence cultural change». ${ }^{\mathbf{1 4}}$ The fixity of a text was not an inherent quality, but rather a "transitive" one, resulting from complex strategies to create "credit" practiced by the different cultural agents involved in the "communication circuit" of the manual printing culture. The early users understood the book as an unfinished, imperfect object. ${ }^{15}$ Can we then identify, in the universe of books of jurisprudence, some strategies to deal with this "uncertainty", managing to transcend it and contributing to the production and dissemination of knowledge? Why did some specific writings manage to do it more than others? In order to address these questions I will examine briefly some ideas related to the construction of the idea of authorship, the role of the agents of the book trade (such as printers and booksellers) and the role of censorship in the diffusion of law books. They all had a significant impact on the creation, production and circulation of legal knowledge, beyond the author's intentions or the originality of his thought. The role of each of these elements may vary according to the case under study. Yet without a reconsideration of these

11 JoHns (1998) 41.

12 On the field of legal history, see the studies of OsLer (1995).

13 Johns (1998) 36. See also the suggestive analysis of McKitterick (2003).

14 Johns (1998) 32-33.

15 MCKitterick (2003) 3 and 95. 
three characters - author, printer and censor, and their creative ways of dealing with the "epistemic indeterminacy" - any history of migration and reception of law books across different places and times would remain deficient.

Several of the issues mentioned above have been largely revisited by book historians in the past decades. Despite the considerable maturity of book history as a "subdiscipline", it probably deserves a few introductory lines in a collection of essays dedicated to another field, such as legal history. As Shelley Rubin ironically points out, «Perhaps an emergent subdiscipline attains maturity at the moment when its adherents assume (usually erroneously) that their colleagues outside the new field understand what it is all about». ${ }^{\mathbf{1 6}}$ Since its birth, usually identified with Martin and Febvre's groundbreaking work L'Apparition du Livre (1958), ${ }^{\mathbf{1 7}}$ this emergent "subdiscipline" has grown through the labor of institutions, annual conferences, a specialized journal and countless publications on a huge variety of topics. ${ }^{18}$ Developing from the crossing of manifold influences, it was possible only «when French cultural historians fused the quantitative book history of the Annales school with an interest in microhistory and mentalité, when German hermeneuticists dismissed the 'affective fallacy' in favor of a reader-oriented Rezeptionsästhetik, and when British bibliographers overturned their peers' view of printing as a discrete constant through which the author's original intentions could be recovered». ${ }^{19}$

The growth of this academic field is evidenced also by the appearance of several companions ${ }^{\mathbf{2 0}}$ and reference works centered on specific territories and nations (such as France, ${ }^{21}$ Spain, ${ }^{22}$ Italy, ${ }^{23}$ Scotland, ${ }^{24}$ Britain, ${ }^{25}$ Amer-

16 Shelley Rubin (2003) 555.

17 BARBIER/MONOK (2009).

18 The work conducted by the members of The Society for the History of Authorship, Reading and Publishing (SHARP), which runs its annual meetings, a specialized journal (Book History) and several discussion groups, was fundamental for this growth.

19 Pethers (2012).

20 Eliot (2007); Suarez (2010).

21 Chartier/Martin (1989-1991).

22 Botrel (2003), Martínez Martín (2001).

23 SANTORO (2008).

24 BeLl (2007-2011).

25 See Morgan (1999-2012). 
ica, ${ }^{26}$ Australia, ${ }^{27}$ Brazil $^{28}$ or Mexico ${ }^{29}$ ) as well as on peripheral printing centers. ${ }^{30}$ Significantly, the discipline counts already with a solid body of canonical texts, reprinted and compiled, for example, in the five volumes of the History of the Book in the West. ${ }^{\mathbf{3 1}}$ Beyond the Western traditions of the book important contributions have also been made, such as the studies assembled in Literary cultures and the material book (which begins with chapters on China, Japan, Persia, India, and Africa), ${ }^{32}$ or the recent reference works addressing the Middle East ${ }^{33}$ and East and South Asia. ${ }^{34}$ Within the Western world, insightful works analyzed the experiences of neglected reading communities, such as those of women, ${ }^{35}$ members of the working classes $^{36}$ or African Americans. ${ }^{37}$ Book history has helped also to offer new perspectives on broad cultural movements such as the French Revolution, ${ }^{38}$ the Enlightenment, ${ }^{39}$ the Protestant Reformation; ${ }^{40}$ or on more recent events such as World War I ${ }^{\mathbf{4 1}}$ and the Second Republic in Spain. ${ }^{\mathbf{4 2}}$

Various attempts to define the subject of book history have been made, describing it as the study of creation, dissemination and uses of script and print in any medium, or "the social and cultural history of communication by print". ${ }^{\mathbf{4 3}}$ Many have harshly criticized its claim of encompassing such a

26 For North America see Hall (2007-2009); some studies on South America in Cátedra/ LóPEZ-VIDRIERo (2004).

27 ARNOLd/Lyons (2001).

28 Hallewell (2005), Villalta (2013).

29 Torre Villar (2009).

30 Rial Costas (2013).

31 Roberts (2010). Cavallo, Chartier (1998) remains indispensable.

32 Eliot (2007) 53-131.

33 Roper (2013).

34 Brokaw / Kornicki (2013), OrSini (2013).

35 JaCK (2012), Cátedra/Rojo (2004).

36 Rose (2001).

37 Cohen/STEIN (2012).

38 Discussing the theses of DANIEL Mornet's Les origines intellectuelles de la Révolution française on the role of the diffusion of books to explain phenomena like the French Revolution, see Darnton (1995) and Chartier (1991).

39 Darnton (1979); Sher (2006).

40 Pettegree/Hall (2004); also Eisenstein (1997).

41 Hammond/ TowheEd (2007).

42 Martínez Rus (2005) 179-203; Martínez Martín (2000).

43 Darnton (1993) and (1982). 
broad spectrum as «the social, cultural, and economic history of authorship, publishing, libraries, literary criticism, reading habits, and reading response» ${ }^{\mathbf{4}}$ and, therefore, the requirement of interdisciplinary skills across different areas of knowledge. However, «books themselves do not respect limits, either linguistic or national», replies Darnton; «[B]ooks also refuse to be contained within the confines of a single discipline when treated as objects of study. Neither history nor literature nor economics nor sociology nor bibliography can do justice to all aspects of the life of a book». ${ }^{45}$

For the purposes of this brief essay, it is relevant to highlight that all these different ways and perspectives within the discipline lead to one basic direction, namely, a focus on the reader as the main performer of every process of reception or creative appropriation of a text in a different context. The various metaphors and interpretative clues suggested for the history of the book in the early modern times, from Chartier's Ancien Régime typographi$q u e^{46}$ to Darnton's "communication circuit", ${ }^{47}$ all point to the perspective of the reader, the final target of the process, the first and last link in the chain of production and circulation of the early modern imprint. The manifold perspectives suggested - the history of reading, ${ }^{48}$ the history of audiences, ${ }^{49}$ the social history of education, the history of the "media" 50 or of the forms of communication, ${ }^{51}$ the diffusion of ideas, ${ }^{52}$ the new sociology of the text ${ }^{53}$ -

44 Greenspan / Rose (1998) ix.

45 Darnton (1982) 80-81.

46 Chartier (1981).

47 Darnton defined the "communications circuit" as a complex web of communication where a series of agents - from author to editor, printer, book dealer, bookbinder, and other intercessors - intervenes, passing from censor up to the reader, in a multifaceted process oriented by social, economic, political and intellectual reasons.

48 Chartier (1995). See the overview of Price (2004).

49 The term was suggested by Rose (2001) 3.

50 See Barbier/Bertho Lavenir (2003).

51 Bouza Álvarez proposes a reconstruction of the debate between writing/reading, seeing and hearing, during the sixteenth and seventeenth centuries in: BouzA Álvarez (1999) 127.

52 As a substitute for the dichotomy between studies of reception, which too greatly emphasized the passive aspect of the process, and the counterproposal of emphasis on the active aspect of transfer, there have been proposed alternatives, such as the notion of diffusion: Helmrath (2002).

53 We refer to the well-known suggestion of Donald F. McKenzie, explained in La bibliographie et la sociologie des textes and in his exemplary study of the typographic transformation 
all consisted of efforts to reorient and broaden the horizon of the old reception theory. ${ }^{54}$

This "methodological thunderstorm" in the book studies of the past decades has had, though, a timid impact on legal history. ${ }^{55}$ Besides certain studies on professional libraries, ${ }^{56}$ editorial projects ${ }^{57}$ and indispensable bibliographical surveys, ${ }^{58}$ not much has been done. ${ }^{59}$ Reversely, book historians have equally paid little attention to the universe of legal books, even if during the early modern age law was still considered one of the three "superior faculties", together with medicine and theology, and the presence of legal books was thus paramount. ${ }^{\mathbf{6 0}}$ This neglect might be explained by the required expertise in legal historical studies or even by a persistent lack of information about the prominent role of the jurists in early modern European learned culture. ${ }^{\mathbf{6 1}}$

The methodological approach suggested in the present study comes from the convergence of book history and the history of law. The two guidelines

offered by the works of William Congreve, where he offers his consideration of the book as "an expressive means": McKenzie (1991) and (1977).

54 The turn from the perspective of the author toward that of the reader was largely developed in the field of literary theory. See the collection of studies bringing together authors such as Jauss, Weinrich, Gumbrecht and Stempel in Holub (1989).

55 António Manuel Hespanha had indicated the consequences of the reorientation of the old history of reception for the history of law in: HespanHa (1990) 187-196. In another study, he tried to establish the relationship between some intellectual shifts in early modern legal theory and the material changes in the layout of early modern legal books (Hespanha [2008]). Also Ranieri had outlined some methodological proposals (Ranieri [1982]).

56 Llamosas (2008); Wijffels (1993); Barrientos Grandón (1992).

57 Both Vallejo $(1998,1996)$ and Conde $(1998)$ offer excellent reconstructions of the editorial projects of Castilian medieval legal texts in the eighteenth century.

58 See the works of Osler, e. g., OsLer (2009).

59 For the medieval jurisprudence there are the studies organized by Colli (2005) and (2002).

60 Clavero (1991) stresses the predominant presence of theological and juridical books in Nicolás Antonio's seventeenth-century catalogue. Most of the reference works on book history quoted above do not have specific chapters dedicated to legal works; a few exceptions are the article of MARSÁ (2004) or the classic Livres, pouvoirs et société à Paris au XVII ${ }^{e}$ siècle (1598-1701) by MarTin (1969). The reference volume Historia de la edición y de la lectura en España does not offer a specific chapter on jurists, although a few data can be found in Buigues (2003c) 424-431.

61 Enciso Recio (2002) is surprised by the presence of legal books in nobility's libraries. 
proposed - the above-mentioned uncertainty of the text and the change of focus toward the reader - might serve as useful instruments to enhance our awareness of the spatial and temporal dimensions and thus reinforce the analytical skills of legal historical inquiry.

\section{Uncertain, immoral, but useful authors}

Early modern readers were not familiar with the idea of modern authorship as an individual ownership protected by copyright rules and based on originality and creativity. Their notion of authorship was probably as indefinite and uncertain as the text itself, and even after the introduction of the copyright it remained essentially fluid and polysemous, attached to diverse individuals and collectivities, such as the publishers ${ }^{\mathbf{6}}$, the compilers, the translators, the adapters or the annotators. ${ }^{63}$ The attribution of authorship was one of the mechanisms used to cope with the uncertainty; however, the attribution to one single author remained problematic since it was often a collective enterprise. ${ }^{64}$ The term "author" was not a synonym for "writer" and it remained apparently close to its original Latin meaning, auctor, which indicated responsibility in a broad sense, from the creation to the promotion of something. ${ }^{65}$ If knowledge was still attached to "authority" (i. e., a superior order of truth), it could not be conceived as an individual property, since it belonged to humanity as a whole. ${ }^{\mathbf{6}}$ The mental experience itself, as Ross explains, was not understood in terms of individual possession. ${ }^{67}$

Illustrative examples of this fluid notion of "author", attached to a broader sense of "authority" in the jurisprudence, is found in two new literary

62 JASZI (1994), 32.

63 See Buigues (2003a), 292-301; Beneduce (1994).

64 Johns (1998), 137.

65 According to Lewis and Short's Latin Dictionary, auctor was «he that brings about the existence of any object, or promotes the increase or prosperity of it, whether he first originates it, or by his efforts gives greater permanence or continuance to it» (LEwIS/ SHORT [1879]).

66 «But knowledge itself was not property. Authority, in this sense, is always proper, always an order of truth gleaned by some individuals perhaps better than by some others, but not possessed solely by any individual. For an individual alone to possess such knowledge would make it purely private knowledge, purely private truth, a blatant self-contradiction» (Ross [1994] 235).

67 Ross (1994), situating the origins of the "possessive authority" in the $12^{\text {th }}$ century. 
genres, cultivated with special interest in German universities in the seventeenth and the eighteenth centuries: those of historia litteraria (literary history) and notitia librorum (knowledge of books). ${ }^{68}$ During this period, several "encyclopaedic" works were published for the use of the jurists: Martin Lipenius' Bibliotheca realis iuridica (1679), ${ }^{69}$ Bukhart Struve's Bibliotheca iuris selecta (1703), ${ }^{\mathbf{7 0}}$ Johann Stefan Pütter's Litteratur des Teutschen Staatsrechts $(1776-1783)^{71}$ and Gerhard Meermann's Novus thesaurus iuris civilis et canonici (1751-1753). ${ }^{72}$ Equally noteworthy are the general compilations, which dedicated important sections to jurisprudence, such as the celebrated Daniel Morhof's Polyhistor literarium. ${ }^{73}$ More modest overviews, such as those written by Beyer, Ludewig or Gundling echoed this general trend. ${ }^{74}$ In France, well-known expressions of this literary genre are the compilations of Taisand and Terrasson. ${ }^{75}$

A good representative of this encyclopaedic spirit of the German scholarship of the time was the jurist Daniel Nettelbladt (1719-1791), active in the University of Halle, mainly recalled as a pupil of Christian Wolff. Besides his studies on natural law, Nettelbladt tried to offer a comprehensive overview of the main authors, schools of jurisprudence and juridical genres from the Antiquity to the eighteenth century, in his Initia historiae litterariae iuridicae universalis. ${ }^{76}$ In these pages, as well as in further writings, ${ }^{77}$ Nettelbladt offers a rich testimony of the mechanisms of the juridical-literary field of

68 Gierl (2001). See also Blair (2010) 166-168.

69 Lipenius (1757).

70 Struve (1756).

71 PÜtTER (1783).

72 Meermann (1751-1753).

73 Morhof (1708).

74 Beyer (1726); Ludewig (1731); Gundling (1707).

75 TaIsand (1737); Terrasson (1750). In the nineteenth century, works such as the Bibliotheque by Camus, annotated by André Dupin in later editions, continued this tradition (Camus / Dupin [1832]).

76 Nettelbladt (1764); in the last section, "Bibliotheca iuridica", he presents several lists of reference works. Nettelbladt's "enzyklopädische[r] Anspruch" is mentioned by RePGeN (2001) 467-468.

77 Such as Systema elementare doctrinarum propaedeuticarum iurisprudentiae positivae Germanorum communis... (NetTelbladt [1781]), and the Hallische Beyträge zu der juristischen Gelehrten Historie, where he reviewed the lives and works of several jurists. 
the early modern era: ${ }^{78}$ its values, rules, communication practices and diverse expressions of the so-called "author-function" ${ }^{79}$ He personifies an important effort to build the "canon" for the respublica iurisconsultorum, in what concerns its historia litteraria, its sources, its subjects and its identity as a discipline.

A significant passage is his attempt to define what a jurisconsult is. Jurisconsultus, the protagonist of the Initia historiae litterariae, says Nettelbladt, is not anyone dealing with "positive jurisprudence". It is someone who chose legal studies ("studium iuridicum") as his main object of learning and whose merits were acquired principally through his erudite written legal production ("potissimum per docta ab eo edita scripta iuridica acquisita"

It is important to note that "potissimum" - principally, particularly does not exclude those who had not left a written production in law and could eventually be considered "jurisconsults" for the purposes of a literary history. This is why Nettelbladt assesses next the critical issue of whether such diverse figures such as Cicero, Paul the Apostle, the biblical King David, Hugo Grotius, Gottfried Wilhelm Leibniz and Christian Wolff could legitimately belong to a historia litteraria iuris. ${ }^{\mathbf{8 1}} \mathrm{He}$ quotes a list of dissertations

78 The analysis of erudite culture (Gelehrsamkeit, Gelehrtenkultur) as a cultural practice, very insightful for the study of the respublica iurisconsultorum, is presented in the volume edited by Mulsow / Zedelmaier (2001).

79 I refer to the locus classicus of the discussion: Foucault's 1969 "What is an author?", in reference to the "death of the author" decreed by Barthes in 1968 (BARTHEs [1968]). For a contextualization of the debate about the death of the author, or his later rebirth, see the results of the symposium of the DFG 2001, in Detering (2001). See also Irwin (2002) and Jannidis (1999). Although part of the debate pertains only to literary theory, one of its outcomes - the contextualization of the distinct expressions of the "author-function" - is also fruitful for the study of the jurisprudential field.

80 Nettelbladt (1764) 25, $\mathbb{} 32$.

81 The complete sentence is: «Non quilibet qui iurisprudentiam positiuam callet, sicque omnino in sensu generali ICtus dicendus, ideo, si in historia litteraria eruditi in classes secundum scientias, in quibus excellent, redigendi sunt, ad ICtorum classem referri debet, eiusque notitia huc pertinent. Requiritur potius, ut quis hic ad ICtos referri possit, eum, praeterea quod sit eruditus, et citra hunc respectum ICtus dici possit, talem esse, qui, nisi sit Polyhistor, studium iuridicum tanquam principale studium elegit, et cuius extant in iurisprudentiam immediata merita, potissimum per docta ab eo edita scripta iuridica acquisita. Unde facile diiudicari potest an, et quo sensu, ad Ictos referri possint M.T. Cicero, Apostolus Paulus, Rex David, Hugo Grotius, God. Guil. Leibnizius, Christianus Wolfius aliique» (Nettelbladt [1764] 25, $\$ 32$ ). 
and orations discussing this topic: from Cornelius van Bynkershoek's De Cicerone non Iurisconsulto and Samuel Stryk's Dissertatio de iurisprudentia Pauli Apostoli to Heinrich Kestner's Dissertatio de iurisprudentia Regis Davidis. ${ }^{82}$

This argument may appear anecdotal to contemporary eyes. Nevertheless, these discussions show the lack of a clear distinction between what we would today consider "author" and "authority". "Jurisconsultus", and thus a juridical author who merited to be included in a historia litteraria iuris, was not necessarily someone who had formally studied jurisprudence and who had left a written work of undisputed authorship. It was also someone whose auctoritas was recognized as in force in the legal field, even if it was somebody who had left only an "oral" register or if the evidences about his existence relied exclusively on the Old or the New Testaments. This was the case of King David, whose authorship of the Psalter was uncertain and controversial: there was a long medieval tradition discussing this topic. ${ }^{83} \mathrm{His}$ doubtful moral virtues (qualified as an adulterer or a sinner) began to be discussed in the theological tradition from the twelfth century on, but the authority ascribed to him as a righteous king and as the voice of God in the Sacred Scriptures qualified him enough to integrate a literary history, according to a widespread opinion. The attribution of more written sources to Paul the Apostle, an educated Roman citizen, seemed less uncertain, such as his letters, added to the Gospels, and the Book of Acts. His past as a sinner before his conversion to Christianity did not matter given his participation in the Sacred Scriptures. However, Paul would not fit exactly in Nettelbladt's definition either. In any case, "author" was such a fluid notion that the "authenticity" of the presumed writings left both by Paul and King David were not brought into the discussion at any moment. In a world where the printing of classical scholarship could be based on "any old manuscript", it is not surprising that almost "any old authority" would still do it too. ${ }^{\mathbf{8 4}}$

82 Nettelbladt (1764) 25-26, $\$ 32$.

83 St. Ambrose, St. Augustine and Cassiodorus had attributed the Psalter to King David, but its authorship offered a major problem (see: MinNis [1984] $43 \mathrm{ff}$.).

84 On the practice of "any old manuscript will do" by the humanist scholars and its equivalent by the modern legal historical scholarship ("any old edition will do"), see: OsLer (1995) 325. 
Concerning Cicero, the debate was certainly subtler, since it referred to the distinction between the officia of the jurisconsults and that of the philosophers under the Roman Republic and the Empire. In the opinion of Nettelbladt, refuting the thesis of Gerhard Noodt, under the Roman Empire both officia were combined, so that prominent figures such as Cicero could be considered jurisconsults and simultaneously orators and rhetores. ${ }^{85}$ Furthermore, these polemics evidence the efforts in delimitating the juridical branch of learning and the standards for communication inside the respublica iurisconsultorum. ${ }^{86}$ To build an idea of authorship and authority was, in sum, one of the strategies to cope with the inherent uncertainty of the printed text and the permeable boundaries of the discipline.

Nevertheless, if ancient authorities raised doubts regarding their written contribution, the modern jurists (recentiores) who had left manuscript and especially printed works offered other kinds of difficulties. As Gutenberg's invention had facilitated the opportunity of being printed, the "authority" of these jurisconsults had to be justified in other ways, relying on fluid criteria such as "usefulness" and "moral virtues". The argument of "utility" is very familiar to the reader of early modern sources, since it appears repeatedly in the prefaces and preliminary texts, and has been extensively documented in other contexts, such as the requests for obtaining printing licenses. ${ }^{87}$ They were not mere rhetorical artifices, but functioned as effective limits for containing and selecting the printed production in general.

These criteria oriented another interesting testimony left by Nettelbladt on how the jurists built the idea of "authorship" in the early modern centuries: his reconstruction of the vivid debate around the literary qualities of the Dutch jurisconsult Arnoldus Vinnius (1588-1657), published hundred years after his death, in $1757 .^{\mathbf{8 8}}$

Nettelbladt's account of Vinnius' life and works would never fit in one of our contemporary journals' sections dedicated to book reviews. His arguments would have appeared exotic to the eyes of our peer reviewers. After a

85 Nettelbladt (1764) 24, $\$ 31$.

86 The term respublica iurisconsultorum, quite widespread at the time, used by NetTelbladt in several passages, is the title of the work of IANUARIo (1733).

87 See abundant examples in García Martín (2003); De los Reyes Gómez (2000) I, 645-666; Conde (2006); Alvarado (2007).

88 Nettelbladt (1757) 638-680. I analyse in detail this debate in Beck Varela (2013) 47-86. 
short reference to Vinnius' noble origins, he deals mainly with the quarrel over plagiarism that accompanied his works and with the supposed injuries to the reputation of celebrated jurists, such as Jacques Cujas.

The victims of Vinnius' plagiarism were presumably his own master, Gerhard Tuning, Bachoff von Echt and Hugo Donellus. All of them had inspired Vinnius' production as an editor, annotator and writer. The main accusers of Vinnius were the Neapolitan lawyer Giuseppe Aurelio de Gennaro, known as Ianuario, author of the Respublica iurisconsultorum and Daniel Morhof in his Polyhistor literarium. Together with the catalogues of pseudonyms and plagiarism, the Polyhistors, typical examples of this already mentioned literary genre dedicated to the notitia librorum, engaged in a public fight against the librorum multitudo, or "superabundance of books", one of the topics that have accompanied the printing press since its beginnings. The need to contain the librorum multitudo functioned as a principle for the selection of books and restriction to written production in the learned world in general. It was of special interest in the case of jurisprudence, where there was a perception that cases of plagiarism were more frequent than in other fields and that the literary production of the jurists was excessive and was deteriorating legal knowledge. ${ }^{\mathbf{9 9}}$ The problem was not only that Vinnius had plagiarized: if he had transcribed entire passages of Bachoff's commentary and additionally had commented unnecessarily the concise Institutes of Justinian $^{\mathbf{9 0}}$, his work, according to Morhof's judgment, was superfluous and should not contribute to the exorbitant number of imprints on the market.

Yet this was not the preponderant opinion. Despite his possible lack of "originality" (Vinnius' debt to Bachoff had even been treated as banal in the reports and protobibliographies of the day ${ }^{\mathbf{1 1}}$ ), the opinion of the majority of the jurists, such as Beyer, who had edited Vinnius' commentary, Ludewig,

89 Morhof mentions that there are many more accusations of plagiarism in the chapter dedicated to juridical books than in the other chapters dedicated to theology and economics (Morhof [1708]). Similarly, Brückner states that the practices of plagiarism and free appropriation of citations were especially accentuated among jurists (BRÜCKNER [1717]).

90 The polemics around the necessity or not of commenting on the Institutes of Justinian was one of the strategies, specific to the respublica iurisconsultorum, to restrain the abundance of legal writings (Beck Varela [2013] 57-64).

91 See the reference to Vinnius' debt to Bachoff in STruve (1756) 982. 
Noricus, Gundling, Gravina or Heineccius, are incontestably positive concerning the erudition and utility of Vinnius for readers. ${ }^{92}$ His merits were appreciated especially for beginners, as in the case of his textbook for the classroom. Utilissimus, amoenus, facilior et luculentior lectu: there is no doubt that Vinnius' ability to handle materials taken from other authors deserved praise. These characteristics associated with the style and utility of Vinnius merited the recognition of the learned community. Nettelbladt invoked them to redeem Vinnius of the heavy charge of plagiarism. Despite the evident exploitation of materials that were not his own, Vinnius' capacity for throwing light on other writers' obscure passages excuses him. ${ }^{93}$ His judgment is categorical: to summarize and synthesize someone else's work does not constitute the crime of plagiarism - a term, which other jurists had not even employed, with the exceptions of Ianuario and Morhof. ${ }^{94}$

In the early modern European context, where the printed book was so widespread, the juridical book is an instrument that has force in offering effectivity - no longer the authority typical of the medieval period. ${ }^{95}$ The very notion of the author in the early modern written culture corresponds to diverse realities ${ }^{\mathbf{9}}$ and has had distinct evolutions in each area of knowl-

92 For the complete bibliographical references see Beck Varela (2013) 47-86.

93 «Endlich kann auch nicht behauptet werden, daß er sich dieses Verbrechens in Ansehung des Bachovis schuldig gemachet, da er seinen commentarium über die Institutionen nicht so wohl ausgeschrieben, ohne ihn zu nennen, wie doch zu dem corpore delicti in diesem Verbrechen nothwendig erfordert wird, als vielmehr, wie Gundling der Wahrheit gemäs urtheilet, in seinem Commentario über die Institutiones dasienige, was Bachov obscur vorgetragen, deutlich gemachet» (NetTelbladt [1757] 677).

94 Ianuario employed it, not on account of Vinnius, but referring to other authors, e.g. IANUARIO (1733), 330.

95 Hespanha (2008) 12-50. Another question discussed on account of the historical forms of the author-function is whether the printing press produced an inversion in the logic of the construction of authorship. Differently to what happened with manuscripts, which were undertaken only if the author enjoyed authority, imprints on their own were turned into a cause of authority: "print ... becomes the cause of authority. Once printed, the text possesses authority, and the writer, however lacking in knowledge or experience, becomes an author who possesses the authority imprinted in the text, whose words must be attended to because some saw fit to print them» (Ross [1994] 242-43).

96 Referrring to the eighteenth century, «[...] la noción de autor corresponde a realidades muy distintas. El autor puede ser el que escribe la totalidad de una obra original, su inventor, y en tal caso adoptamos la definición que hoy suele sobreentenderse. Pero también puede ser un comentarista, un traductor, un adaptador o el que realiza una compilación, y en la mente de la época dominaba más bien este concepto amplio de autor [...] 
edge. ${ }^{97}$ This discussion surrounding the "authorship" of Vinnius is illustrative of two positions in conflict with the definition of the "author-function" which operated in the juridical discourses of that time. From a predominant point of view, the image of the copyist and compiler still received a positive evaluation. The collective, corporatist or "collaborative" notions of writing, still persistent in the origins of the modern copyright, ${ }^{98}$ are prevalent also among the members of the respublica iurisconsultorum. Ludewig argues that the plagiarism between Hotman and Balduinus came to benefit the reader. ${ }^{99}$ In eighteenth century Spain, similar debates or doubts surrounded the publications of the Sacra Themidis of Franckenau (or Juan Lucas Cortes'), De Lolme or the free translations of Raynal written by the Duque of Almodóvar. ${ }^{100}$ Far from the late nineteenth-century conception of authorship, the weak auctoritas or acceptance of Vinnius and other jurists of the day appeared to emanate fundamentally from the values recognized by the community - of effectiveness, utility and vague notions of style that explain the typographic success that his works had merited. ${ }^{101}$ In spite of the absence of criteria like that of romantic "originality" - a word that neither Nettelbladt nor the other mentioned authors use - there is, in contrast to the laxity of the predominant opinion, a more rigorous point of view, represented by

dicho de otra forma, es un autor cualquier individuo bajo cuyo nombre se publica un impreso». Buigues (2003a) 292. For a typology of authors, see Glendinning (1983) 4, 43-45.

97 One of the fruitful critiques of Foucault's approach is precisely the one that refers to an inadequate generalization of history that had situated the emergence of the author-function as a limit of discourses in the disciplinary society of the eighteenth century. See Chartier (2000) 90-105 and (1994) 41-67.

98 See Woodmansee (1994) 15-28. For the genesis of copyright in France, see Hesse (1991); for England see Rose (1993). For a legal historical overview of copyright law in the nineteenth century in Europe: Mazzarella (2010).

99 «Hotomannus Balduino, amico et praeceptori, sua commenta primus transmisit. Respondit hic illi, sibi institutum esse simile. Utrique igitur eadem laude digni esse, videntur. Sed malem Hotomanno carere, quam Balduino. Felix tamen lector utrisque» (Ludewig [1731] 48).

100 See Clavero (1993) and (1992); and Vallejo emphasizing that the appropriation of the text of Raynal by the Duque de Almodóvar exceeded the boundaries of the "translator" even for the broad criteria of the day (Vallejo [2000] esp. XXXIV-XXXV and XXVII). Muñoz de Bustillo mentions the appropriation of the manuscript of Andrés de Pozas by Juan Gutiérrez (Muñoz de Bustillo [2006] CXI-CXVIII).

101 Nettelbladt (1757) 657. 
Morhof or Ianuario. ${ }^{102}$ Both illustrate a new and growing sensibility toward literary plagiarism and other practices of simulation and dissimulation so common in the learned community. The alert pages of the Polyhistors as well as the long catalogues of pseudonyms, claiming for vigilance among scholars, fought against these practices. ${ }^{103}$

The problem of literary plagiarism had begun to be a theme of discussion among jurists. The brief work by Jakob Thomasius, De plagio literario (1673), followed by the accessiones of 1679 (which offered a supplement to the catalogue of plagiarists and an alphabetic index $)^{104}$ is perhaps one of its first systematic treatments. The work was well received at the time and inspired other authors, ${ }^{105}$ such as a dissertation dedicated especially to De plagio literario in studio iuris. ${ }^{\mathbf{1 0 6}}$ Among the theses of Thomasius, developed under the form of theorems, are the definition of plagiarism as a lie, a question belonging to the moral sphere; and the distinction between judicial and extrajudicial plagiarism, whose persecution would take place exclusively in the realm of the orbis eruditus. ${ }^{\mathbf{1 0 7}}$ Thomasius offered a curious catalogue of the universal history of plagiarism, including such disparate authors as Greek and Latin classical authors (Homer, Sophocles and Aristotle), Ambrose, Gratian, Isidore of Seville, and hitherto jurists like Cujas. His notion of plagiarism is a wide-ranging and malleable one, not clearly distinct from other forms of literary appropriation often used. The index itself is titled Index plagiariorum, vel quasi. Nevertheless, there is no doubt that this work shows the development of a new sensibility or consciousness relative to the strengthening of the "author-function" as a limit to the appropriation and circulation of discourses.

Another curious aspect of Nettelbladt's portrait that would not fit in a contemporary biographical sketch is his description of Vinnius' moral vir-

102 In Spain, Gregorio Mayáns represented a critical voice, although more flexible than Morhof or Ianuario.

103 Mulsow (2006).

104 Thomasius (1673); Thomasius (1679). Jakob Thomasius is the father of Christian Thomasius, who precociously had also contributed to the debate with a definition of decorum (JaUMANN [2000] 72).

105 SChWARTZ/STEMPEL (1701).

106 BRÜCKNER/BROCRIS (1717).

107 For the association between the definitions of Thomasius and an incipient public opinion see Jaumann (2000). 
tues. This attention to moral virtues appeared frequently in the jurisconsults' protobiographies of the time: Gundling, for example, mentioned Wesenbeck's "castitas and fides coniugalis" against the accusations of philandering. ${ }^{108}$ In the case of Vinnius, the reviewer focuses his attention on the moment of his death. His death was not especially tragic, nor was it surrounded by extraordinary circumstances: he died at home, attended by a few friends and colleagues (his wife and four children were already deceased), after a short period of illness. However, Vinnius had supposedly faced that difficult moment of his illness with remarkable serenity. In Nettelbladt's outline, the exaggeration of Vinnius' virtues, in the parameters of Christian morality, comes as a complementary support to his literary merits. This double facet of the juridical author demonstrates, moreover, the indistinctness between the intellectual and the moral aspects. ${ }^{109}$ This indistinctness seems to have been constitutive of the representations of the auctor, which had endured since the medieval period. ${ }^{\mathbf{1 1 0}}$ In his Systema elementare doctrinarum propaedeuticarum, Nettelbladt wrote about the "animae conditio" of the jurisconsults. He said that their anima was characterized by moral and intellectual vices and virtues. Their intellectual virtues are the scientia and the capacitas. The ancient jurisconsults excelled in both, but the medieval ones excelled rather in capacity than in knowledge («scientia tanta non fuit, magna tamen omnino fuit eorum capacitas»). ${ }^{\mathbf{1 1 1}}$ The recent ones (recentiores) have great intellectual virtues, like those of the ancient ones («Recentiores iurisconsulti vero iterum ad veterum iurisconsultorum gloriam adscendunt»). However, many of the recentiores do not excel in moral virtues («Quoad vitia moralia virtutesque morales, Iurisconsulti, praesertim recentiores, apud multos quidem male audiunt; ast et hic valet, sunt boni mixti malis»). ${ }^{\mathbf{1 1 2}}$ In this sense, the undoubted moral virtues of Paul the Apostle

108 Gundling (1707) III, 214-274, on 246.

109 Stating the lack of distinction between the physical aspect and the moral or intellectual one of the act of reading in the early modern period, Robert Darnton underlines the "physical element" of the act of reading, which is expressed in metaphors like "readingas-digestion" or "reading as spiritual exercise" (DARNTON [1990] 172).

110 ZimMERMANN (2001) 7-14.

111 Nettelbladt (1781) $\$ 200$.

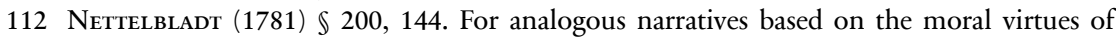
judges and lawyers see the description of the perfectus advocatus by CunHa (1743) and the references quoted by GARRIGA (2004) on the literature of the iudex perfectus. 
(even though he had been a sinner before the conversion) have certainly contributed to assure his status as a jurisconsult.

In sum, the recourse to moral virtues, besides being inseparable from intellectual merits in the mentality of the time, helped to justify or to reinforce certain figures of authorship.

If we had asked an early modern jurist how to define a legal auctor in the science of law, he would probably have answered that it was someone who had contributed to knowledge in the legal field, even if there were doubts about his written legacy. He would have probably said, too, that an auctor was someone who had contributed a useful work (even if he had benefited from previous jurists' writings) and who was, preferably, a virtuous man, since both reading and writing embodied a certain notion of Christian morality and were not detachable from other moral actions. This fluid and collaborative notion of authorship, which was under construction during the early modern centuries, was one of the ways of coping with the uncertainty of the early printed text. Without these notions in mind, we will be able to understand neither the process of creation nor the diffusion of the early modern legal literature.

\section{Printers and censors: manufacturing credit, spreading uncertainty}

It is an obvious fact that printers and censors alike determined decisively how early imprints were created and how they circulated. Nevertheless, it is not usual to consider the ambivalent consequences of their actions. While they were responsible for "manufacturing credit, ${ }^{113}$ i. e., for fighting against uncertainty and building strategies to create text reliability, they were also responsible for its reverse effects: the increase of the uncertainty of the printed texts.

Printers - or, more broadly, the various agents associated with the book trade $^{\mathbf{1 1 4}}$ - were a fundamental piece in the "communication circuit". They

113 «Printers and booksellers were manufacturers of credit» (JoHns [1998] 31).

114 Concerning the printer, editor, and the book dealer - key figures in the production of the early modern book, often united in the same person - see the synthesis of Jaime MolL (2003) 77-82, and the remarks of Mario InfelisE: «Il libraio, inoltre, che, più di qualsiasi altro operatore del settore, stava avvicinandosi alla figura del moderno editore. Era solitamente lui ad elaborare una propria originale linea editoriale, a tenere i rapporti con gli autori, a curare la commercializzazione dei libro all'interno e all'estero, ad organizzare la 
were virtually responsible for any element of a printed sheet, for it was impossible to isolate «a consistent, identifiable, immutable element attributable to the individual author». ${ }^{\mathbf{1 1 5}}$ They were crucial in shaping what may be defined as "external" strategies - such as establishing networks, negotiating with the authorities and supporting important editorial projects - as well as "internal" aspects, such as the process of adaptation of legal texts, the creation of new meanings, new uses and even a new target public for certain works or genres.

Printers and book traders in general saw the field of jurisprudence as an attractive market, especially the branch of the reprints of works of previous decades and centuries. The Benedictine writer Martín Sarmiento complained that in the 1720's, the book dealers in Madrid «no se extendían más que comprar o vender [...] libros triviales y comunes; y el que mas, á traficar en libros facultativos que llaman de pane lucrando, v. gr. de Medicina, Leyes y Teología» ${ }^{\mathbf{1 1 6}}$ and the reprints appeared to have dominated the Spanish legal culture in this period. ${ }^{\mathbf{1 1 7}}$ They supposed a guaranteed readership and required lower taxes to obtain printing licenses, ${ }^{\mathbf{1 1 8}}$ representing certainly a

rete dei corrispondenti» (InfELISE [1989] 138). Johns refers to the "Stationers", which were formally all men and women concerned in the book trade and members of the Company of Stationers, recognized by Queen Mary in 1557. However, "Stationer" meant not only a corporate affiliation, but a distinctive cultural identity that bound those who were involved in the printing craft (JoHns [1998] 59-60).

115 Referring to the Stationers, Johns states that «[in] managing publications, Stationers, and often booksellers in particular, controlled events. The practices and representations of their domains affected every character and every leaf of their products. Isolating a consistent, identifiable, and immutable element attributable to the individual author would be virtually impossible in these circumstances. Attributing authorship was thus intensely problematic for both contemporary and future readers. A priori, virtually any element in a work might or might not be the Stationers' responsibility, in virtually any field of writing» (JoHns [1998] 137).

116 Martín Sarmiento, Pe., "Reflexiones literarias para una Biblioteca Real, y para otras Bibliotecas publicas, escrito [...] en el mes de diciembre de 1743" (1743) 99-272, on p. 132 (apud JuÁrez Medina [1988] 193).

117 Tomás y Valiente (1988) 369. See the comprehensive overview by Juárez Medina (1988). Buigues stresses the prevalence of reprints among the books of jurisprudence: Buigues (2003b) 318.

118 There were less expensive taxes for the re-impressions (De Los Reyes Gómez [2000] t. I, $513,615)$. Esteban Conde analyzed the difficult formalities faced by those who wanted to become juridical authors in eighteenth century Spain (Conde [2006]). The Juez de Imprentas in his Informe of 1752 prescribed penalties for the reprints without licenses. These 
less risky enterprise. ${ }^{119}$ Although the history of diffusion is not a synonym of a recounting of reprints, ${ }^{\mathbf{1 2 0}}$ they can offer a good indication of the predilections of the readers and were mainly conceived for them, being in most cases completely independent from the author's intentions. ${ }^{\mathbf{1 2 1}}$ The most widespread legal genres of the early modern centuries, for instance, circulated thanks to the reprints undertaken by important typographic houses.

A good sample of this reprinting phenomenon are the names quoted in the introductory lines, those of Salgado de Somoza and Juan Gutierrez, quoted in Meerman's Thesaurus as well as in Nettelbladt's Initia historia litterariae. The re-impressions of their works were issued in most cases long after their death by the printing shop of Anisson in Lyon, ${ }^{\mathbf{1 2 2}}$ which was an important exporting center linking the northern and the southern markets. Especially from the seventeenth century on, the Iberian book market was attractive to the printers of the North, since they scarcely faced there strong local competitors. ${ }^{123}$ This family (the Anissons and their associates, Duperron and Posuel), which had agents in the Peninsula, engaged in printing Spanish and Portuguese jurists and shaped conclusively their European diffusion. The Anisson family printed a considerable number of works in vernacular - libri Hispanici, Italici- besides several juridical, theological and philosophical works printed in Latin. Among these, many Portuguese and Spanish jurists went through the Anisson presses, such as Solórzano Pereira, Castillo Sotomayor, Agustín Barbosa, Domingo Antunes Portugal, Pereira e Castro, Melchor de Valencia, Lorenzo Matheu y Sanz, Manuel González

licenses for reprints sometimes encompassed the privilege to reprint various works and authors (JuÁrez Medina [1988] 140-141).

119 «The reprint figures are especially valuable, for they tend to reveal more accurately the activities and predilections of the reading public than do the first edition figures. A first edition is almost always a commercial risk for the publisher. The reprint, conversely, is almost always a guaranteed success, for it is generally prompted by previously demonstrated enthusiasm on the part of the book-purchasing public» (BEARDSLEY JR. [1986] 14-15).

120 Many reprints of Vinnius' works were in reality hiding old unsold sheets under a renovated title page (Beck Varela [2013] $105 \mathrm{ff}$.).

121 Leah Price, noting that the "reprint series" are based not on the author's identity anymore, but on the readers' identity (PrICE [2004] 311-313).

122 Salgado de Somoza's Tractatus was reprinted by Anisson in 1647; Juan Gutiérrez in 1661. On Gutiérrez see Alonso Romero (1997).

123 See Martín Abad (2003); Péligry (1990). 
Téllez and Gaspar Rodríguez. Some of the most popular legal works of the time consolidated their presence in the Peninsula and beyond its boundaries through the Anissons' editions, such as Gil de Castejón's Alphabetum iuridicum, the additions to the glosses of Gregorio López by Hermosilla; or the notes to Antonio Gómez written by Ayllón Laynéz. ${ }^{124}$

If we turn it the other way round - to Northern jurists who managed to trespass the Pyrenees, after the Protestant Reformation - a few more requirements for the cross-bordering were necessary. Historians have looked at the new political and religious boundaries built after the Reformation sometimes as porous and sometimes as impermeable, as meeting points as well as impervious walls. ${ }^{125}$ The dichotomy between porosity and impermeability might not be the most appropriate perspective to capture a broad view of the effects of this important cultural event. Even if the new political and religious divisions were not always perfectly aligned, ${ }^{\mathbf{1 2 6}}$ they have undoubtedly shaped a new dynamic for the production and circulation of knowledge, including the jurisprudential.

One of the core mechanisms in this new dynamic, which can be paradigmatically seen as a "boundary" and a "passport" for written production, is the Index of forbidden and expurgated books. Many of these Indexes were published throughout Catholic Europe, from Rome to the Spanish Low Countries, ${ }^{\mathbf{1 2 7}}$ although book censorship is not in any case a Catholic exclusivity. ${ }^{\mathbf{1 2 8}}$ They consist of the most visible instrument of the well-known politics of censorship ex post undertaken by the ecclesiastical authorities.

124 See the Bibliographia Anissoniana, a catalogue of works available for sale in his bookstores of Paris and Lyon printed in 1702 (including also imprints by other shops): libri hispanici in pp. 455-78; in the pp. 163-232 we find the section dedicated to the libri iuridici. For more information about the family of printers, see RenOuARD (1995) 6.

125 About the ambivalence of the "frontier", which «[...] implique a contrario le fait du passage et celui du passeport, c'est-à-dire l'idée du voyage, de la rencontre de la différence de l'autre, même s'il s'agit d'une rencontre controlée, limitée, parfois réservée à quelquesuns, rencontre légale toutefois», see Garrisson (1992) 9.

126 Pastore refers to «il problema della coincidenza fra i confini territoriali ed i confini religiosi, ovvero del loro mancato allineamento» (PAstore [2007] 15).

127 See the eleven volumes of the collection Index des libres interdits, directed by MARTínez DE BuJANDA and completed in 2002.

128 See, e.g., Jostock (2007). 
No matter how paradoxical it might appear prima facie, an author's presence in the Index functioned as a "visa" for crossing the Iberian frontier. ${ }^{129}$ Consider for example one of the most widespread legal genres, mentioned in our preliminary lines - that of the commentaries on the Institutes of Justinian. The four representatives most frequently quoted in the sources of legal education, and likely abundant in today's libraries, all entered the Spanish catalogues. These were Joachim Mynsinger (1514-1588), Johannes Schneidewein (1519-1568), Matthaeus Wesenbeck (1531-1586) ${ }^{\mathbf{1 3 0}}$ and the already quoted Arnoldus Vinnius. Additionally, all of them deserved printed versions adjusted to the Inquisition standards, which facilitated their voyages around the Spanish and Portuguese territories. ${ }^{\mathbf{1 3 1}}$ The same did not happen to other authors of similar works who had entered the Index as well. ${ }^{\mathbf{1 3 2}}$ The Index itself was a business opportunity for printers, who hurried to issue modified versions. ${ }^{133}$ It was also an occasion for faked announcements of adaptation, spreading the uncertainty. The multiple forms of "piracy" - such as these false promises, false places of imprint, plagiarism in its broad meaning and other kinds of text corruption - were not seen as accidental, but commonplace and even inherent to the print culture of the time. ${ }^{134}$ As noted above, printed books, even when transcending by far their immediate spatial and temporal circumstances, were not self-evidently creditable: when taking them in their hands, readers considered the reliability of the people and places involved in its fashioning. ${ }^{135}$

129 The Italian case is different, since the Roman authorities failed to publish expurgatory Indexes (see the analysis of SAVELLI [2011]).

130 Wesenbeck was quoted especially for his Paratitla, a brief commentary on the Digest and the Codex, besides his commentaries on the Institutes.

131 See for example Mynsinger (1691) and Wesenbeck (1639); copies of their works abound in Spanish and Portuguese libraries.

132 There are no expurgated editions, for example, of the commentaries on the Institutes of Johann Harprecht, Gerhard Tuning, or François Hotman.

133 In the Italian territories the situation was not different: see countless examples in SAVELLI (2011) $16 \mathrm{ff} ., 23 \mathrm{ff}$.

134 Johns (1998) 171; McKitterick (2003). A curious example of the malice of the printers is observed in the Nuremberg edition of 1734, whose title page falsely announced inexistent expurgations (BeCK VARELA [2013] 111).

135 JoHns (1998) 62. "Living in a world where printed materials could not necessarily be trusted, contemporaries developed a wide range of techniques, social, material, and literary, to affirm and defend what they claimed to be knowledge. In particular, they were well aware of the insuperable independence of readers» (JoHNs [1998] 378-379). 
Other jurists, who had possibly demonstrated a non-negligible ability in explaining the Institutes, had not met at least one of the conditions for a wide diffusion: whether the presence in the Index (as an expurgated but allowed reading) or the help provided by a corrected printed version. Both factors contributed to multiply the chances of being further reprinted and imitated by other printing shops.

Both conditions concurred, for example, in the case of the Dutch jurist Arnoldus Vinnius, whose unprecedented popularity surpasses that of any other jurist during the eighteenth century in Spain. The extraordinary diffusion of his works is due mainly to the conjunction of five factors - all of them distant from the authors' intentions - and could not be understood without considering the premises discussed here, such as the fluid notion of authorship and the decisive role of printers and censors. The first decisive factor was the inclusion of his Commentarius academicus et forensis, printed in Leiden in 1642, in the Spanish Index librorum expurgatorium et probibitorum of 1707 (the censored passages were amplified in the 1747 Index), offering a safe port for readers and book traders. Second, the initiative of a printer like Anisson, alert to the demands of the jurisconsults and ready to take typographic risks. He had already suppressed some of Vinnius' heterodox sentences in previous editions of his Commentary (e. g., in 1683) and in 1708 a more reliable edition according to the new Index of 1707 was issued. ${ }^{\mathbf{1 3 6}}$

Third, the spread of Anisson's model in other printing shops, such as that of Francisco Lasso's in Madrid in 1723 (being probably the first imprint of a Protestant jurist in Spain). The existence of a trustworthy corrected edition facilitated considerably the printers' work. Other printers reproduced this model in subsequent editions, such as the Institutes of the Salamancan Professor Antonio Torres y Velasco, printed in 1735 without the acknowledgement of Vinnius' authorship on the title page. ${ }^{137}$ By the end of the century, with the closing of the Spanish border to the European book mar-

136 Anisson prepared two different versions of the 1708 edition: one of them was adapted to the Index prescriptions, indicating Vinnius as auctor damnatus in the title page and suppressing the forbidden passages (BECK VARELA [2013] 113-121).

137 Torres y Velasco (1735); see the critical remark by Sempere y Guarinos (1785) II, 240. 
ket, ${ }^{138}$ the reinforcement of civil censorship ${ }^{139}$ and the reprints of his commentaries in further expurgated versions (now also with the precious notes regarding the sources of the ius patrium), Vinnius' hegemony in the Spanish legal schools was assured.

A fourth reason that helps to understand his predominance over other similar commentators is probably the new "reading protocol" (Chartier) implemented by Anisson in Vinnius' editions: he added Vinnius' Notae (published originally in 1646 as a separate work) alongside the Commentarius and Justinian's text in a renewed page layout. ${ }^{140}$ The posterior editions of the Commentarius reproduced this new model, especially suitable for the classrooms and for the kind of reading practices developed by the students, based on techniques of memorization of excerpts and on constant repetition. Readers ended up by considering this redundant model as intrinsically attached to Vinnius' commentary. ${ }^{141}$ It helps to explain why he was known as the "oracle" in the teaching of law in Spain until the beginning of the nineteenth century. ${ }^{\mathbf{1 4 2}}$

Finally, the incorporation of the sources of the ius patrium in the late eighteenth-century Spanish editions - through notes, footnotes and headings indicating the corresponding passages in the Siete Partidas or in the

138 This is one of the main features of the measures undertaken during the reign of Charles III. See the Privilegio concedido a la Compañía de Impresores de Madrid y demás mercaderes de libros, para que no pudiesen introducir en España ni en América los libros extranjeros reimpresos en España, Madrid, 29 de noviembre de 1763 (DE los Reyes Gómez [2000] I, $553)$.

139 See García Martín (2003).

140 The expression "protocol de lecture" is Chartier's (see Chartier [1992] 55, 59). He explains that «Le plus souvent, dans l'édition ancienne, ce qui est contemporain du lecteur n'est pas le travail d'écriture mais celui d'édition, et la 'lecture implicite' visée par le libraire-imprimeur vient se superposer, parfois contradictoirement, au 'lecteur implicite' pensé par l'auteur. Les dispositifs typographiques importent donc autant, voire plus que les 'signaux' textuels puisque ce sont eux qui donnent des supports mobiles aux possibles actualisations du texte, puisqu'ils permettent un commerce perpétué entre des textes immobiles et des lecteurs qui changent, en traduisant dans l'imprimé les mutations des horizons d'attente du public, en proposant de nouvelles significations, autres que celles que l'auteur entendait imposer à ses premiers lecteurs» (CHARTIER [1985] 62-88).

141 See the preface of the Spanish translation of the Commentarius, printed in Barcelona in 1846 (Vinnius [1846]).

142 Peset (1975) XLII-XLIII. 
Nueva Recopilación - played also a decisive role for the consolidation of his presence among students and professionals. The scheme of the standard Justinian text illustrated by the local law perspective, as Birocchi highlights, explains the European editorial success of authors like Vinnius, Voet, Huber, and Heineccius. ${ }^{\mathbf{1 4 3}}$ The promoters of new editions of Vinnius, such as the jurists Bernardo Danvila (1700-1799) and Juan Sala (1731-1806), invoked the "utility" of the ius patrium's notes, which were of increasing importance in the eighteenth century. Juan Sala even engaged personally in strategies to assure a portion of the market for his Vinnius castigatus, threatened by other competing editions. The Council of Castile finally attended his claims. ${ }^{144}$ If even a genius like Galileo had to struggle to assure readership and publication of his works, ${ }^{\mathbf{1 4 5}}$ why should a poor commentator of Justinian's Institutes do less?

143 He states, concerning widespread legal works, such as those of Vinnius, Voet, Huber or Heineccius, «[...] che sembrano proporsi nel solco della continuazione di una scienza comune europea modulata sulla compilazione giustinianea. Ma il successo di questi giuristi si spiega innanzi tutto con la capacità altissima di 'insegnare un metodo per argomentare giuridicamente e poter formulare decisioni': il che sta a significare che essi, raffinatissimi per strumentazione storico-filologica, non venivano letti come se insegnassero il diritto immediatamente vigente. Si spiega poi con la prospettiva modernizzante e per la diretrice 'patria' che essi aprivano attraverso la considerazione dei mores e del ius hodiernum» (BIROcCHI [2006] 29; in quotation marks, citing Van den Bergh).

144 Torremocha summarizes this episode: «La obra de Vinnio fue reimpresa en Valencia por Benito Monfort, y el Consejo determinó en 8-X-1779 que los catedráticos de Instituciones Civiles explicasen y enseñasen por esta edición. Esto dio lugar a que el Dr. Juan Sala, catedrático de Instituta en la Universidad de Valencia dirigiese una representación al Consejo (3-XII-1780), manifestando el agravio que se le hacía, pues él había publicado su obra Vinnius Castigatus. Si todos los estudiantes estaban obligados a comprar la edición de Monfort, que había establecido unos precios fijos - 45 reales en papel y 50 encuadernados - no podía vender sus ejemplares hechos con fin académico. Esto determinó que el Consejo tras realizar una consulta - a tres Fiscales y al abogado del Consejo y Catedrático de Prima de Granada, Pedro José Pérez Valiente - accediese a que se utilizasen cualquiera de las dos obras. A.U.V., Libro de Claustros n ${ }^{\circ}$ 18, fol. 544, 26-X-1781; Libro ${ }^{\circ}$ 509, 17-X1781» (Torremocha [1993] note 189, 78-79). Another strategic effort of the author is expressed in the separate publication of his notes to the second edition of the Vinnius castigatus, as a complement for those readers who already possessed the first edition (SAla [c. 1786]).

145 Biagioli (1993). 
Vinnius' case illustrates remarkably well all possible factors involved in the diffusion of an early legal imprint - material conditions, local market's limitations, social networks, additional notes on local law, censorial prescriptions, printers' creativity, and the potential impact of their innovations in expanding the target public. All these elements intend to enrich our comprehension of diffusion processes, far from trying to reduce the book to the condition of a mere material commodity, as if it did not carry out written words of cultural and social relevance. This elementary criticism of material approaches has been the object of vibrant debates. ${ }^{\mathbf{1 4 6}}$ For what concerns legal history, the focus on readers' demands and experiences, and the awareness of the "text indeterminacy" as methodological tools, remind us of the primary assumption that legal knowledge, as any other kind of learned knowledge, is always produced in a certain time and a certain space. As in the academic world nowadays, a complex set of conditions defines the production and dissemination of knowledge. ${ }^{\mathbf{1 4 7}}$

Beyond Vinnius' particularities, it is possible to state that the first two conditions mentioned above - presence in the Index or in other censorship edicts, and the existence of a corrected printed version - might have assured or at least remarkably enhanced the chances of crossing the Pyrenees' borders.

It does not mean, notwithstanding, that all jurists were censored the same way, nor that the expurgated versions would assure a serene trajectory among the Iberian readers. The Index librorum prohibitorum et expugandorum did not guarantee, in any case, uniformity or stability in the practice of censorship. Once again, it is necessary to face the intrinsic indeterminacy of the printed text, as well as the readers' perspective. The text itself was conceived as incomplete, unfinished; as a space of readers' interventions and interactions with the text through various means: punctuation, marginalia, correction of typographic errors, translation of Greek characters, and hand censorship. McKitterick reminds us that «[a]s an activity, censorship introduces its own instability». ${ }^{148}$ Since it depended on individuals inasmuch as on the

146 Gordon (1998); EISENSTEIn (1997).

147 Bourdieu (1984).

148 MCKitTerick (2003) 152. 
official prescriptions, censorship multiplied an awareness of uncertainty regarding the printed text. ${ }^{149}$

The sources of uncertainty in the practice of censorship relied on the impracticality of the enterprise itself: the civil and ecclesiastical authorities were well aware that it was impossible to control every printed piece issued by Protestant or other heterodox hands. Once the censorship commissions had carefully examined and expurgated one edition and it had entered the Index, none could guarantee that the new ones would obey its model. On the contrary, readers and censors alike were conscious of this permanent difficulty. ${ }^{150}$ Moreover, the Index contained numerous typing errors, which in some cases hampered the labor of expurgating. ${ }^{151}$ Another cause of "anxiety" ${ }^{\mathbf{1 5 2}}$ was the difficult access to the Index, expressed in the endless complaints about the scarcity of its copies. ${ }^{153}$

The forms of hand expurgation were diverse: by excision, by crossing through, or by more vigorous and careful ink deletion. Readers made also often very smooth and transparent signs, which did not impede the reading, or solely added marginalia on the side of the prohibited passages, such as

149 MCKitTerick (2003) 153.

150 See for example the testimony of a reader who took his notes on a copy belonging to the library of the University of Seville: «Esta impresión de Madrid del año 1723 no es la que se manda expurgar: por que exstá [sic] expurgada. La impresión de Leon del año 1666 por Lorenzo Anison es solamente la que debe expurgarse, como puede verse en el $1^{\circ}$. tomo del expurgatorio fol. 22 verbo Arnoldus Vinnius J. C. etc.a. esta impresión está conforme a la de Valencia del año 1767. Dedicada al Exmo. Conde de Aranda». It is a copy of the Seville University Library, which in its day belonged to the bachiller Francisco Xavier Peñaranda y Velasco, Fernandez de Reguera (VInnius [1723-1724]). The same uncertainty persisted 20 years later: in a copy of the second edition of Danvila in 1786 which continued in use until at least 1844, the owner indicated that «[...] esta obra del Vinnio está mandada expurgar por la Inquisicion, según el expurgatorio de 1747 pag.25: pero como esta edicion fue impresa en 1786 en regular que se arreglaria a aquella disposicion, sin embargo el sujeto que use de ellos devera leerlos con cautela y examinar si se han o no expurgado como ali se manda». (This copy belongs to the library of the Colegio de Abogados de Barcelona (Vinnius [1786]). Enrique Gacto relates similar uncertainty concerning the editions in his study on Gonzalo de Illescas' work (GActo [1992] 23-40). See SAvelli (2011), $22 \mathrm{ff}$.

151 Beck Varela (2013) 313.

152 "Censorship is a measure of the anxiety a given culture has about the impact of books on its society» (ELIOT [2007] 27).

153 The Index of 1790 mentioned this difficulty (Index [1790] XIII). Martins alludes to the troubles in finding the prohibitory edicts in the Portuguese context (MarTins [2005] 825). 
caute lege. ${ }^{154}$ The very same edition of the same legal author could be read and censored in various ways, especially amidst a qualified readership such as that of the professional lawyers and scholars. The universe of legal books, as other books qui de religione non tractant, were under a less rigid suspicion. ${ }^{155}$ They counted on a reading public in many cases sufficiently prepared to confront heterodoxy, many of them usual receivers of special licentiae legend. ${ }^{156} \mathrm{~A}$ respectful reference to Hugo Grotius by a Neapolitan censor («ex Etherodoxis Clarissimus Grotius») is a good example of this mentality. ${ }^{157}$

Not only did the official censorship criteria vary substantially within the bounds of Roman Catholic Europe - the different expurgations of Vinnius proposed in Rome, Naples and Madrid are a good example again - but the ways in which readers approached them changed enormously. The copies of early legal imprints remaining in today's libraries vary from the complete absence of expurgation (besides the existence of totally forbidden works) to the other extreme, that of the presence of expurgations spontaneously added by the reader, i. e., the deletion of passages that were not strictly forbidden in the Index. This practice of spontaneously adding censures on legal books

154 «The only possible conclusion to be drawn about the textual transparency of many expurgated texts, and about the ease with which forbidden texts can be viewed, is that simple demarcation was deemed to be sufficient: the faithful might, as it were, look over the boundary, but they might not acknowledge what they might see, or use it in their own private devotions or in their intellectual life» (MCKITTERICK [2003] 160).

155 The Tridentine Index of 1564 (which had smoothed the more rigid tone of the previous Index, of Paul IV), in its second rule, received in later catalogues, determined that books written by heretics that did not deal with religion, but with other subjects, should be recalled and examined by theologians named by bishops and inquisitors (in the edition of Martínez de Bujanda [1984-2002] VIII, 150; see rule 3 of the Indexes of 1583 and 1640; second rule of the 1632 Spanish Index; also in the Portuguese expurgatory Indexes of 1564, 1581 and 1624). In the Spanish version «[...] Los libros de los Hereges, que de proposito tratan de Religion y puntos controversos de ella, se prohiben del todo. Mas bien se permiten los que no tratan de ella, siendo primero examinados y aprobados por Teologos pios y doctos, por nuestro mandado, como son muchos contenidos y permitidos de la primera Clase de este nuestro Catalogo» (Reglas, mandatos y advertencias generales, III, reproduced in Index [1707] s.p.; Índice [1790] XVI).

156 See Frajese (2000) 210; Savelli (2011) XX.

157 In the preliminary notes to Vinnius Commentarius printed in Naples in 1772, censorship note nr. IV, p. 50; reprinted in 1804 (VInNius [1772]). He mentions Grotius' theses in other passages of his notes. 
persisted until the end of the eighteenth century. ${ }^{158}$ This is because the Index itself was not understood as a complete, finished work, but as one incomplete artifact open to the readers' collaboration.

Besides the evident difficulties in controlling the arrival and diffusion of imprints, ${ }^{159}$ it is important to consider that the norms of the Index librorum were not understood as absolute prohibitions (as were not, in general, the norms of the Old Regime), ${ }^{\mathbf{1 6 0}}$ but rather as suspensions of a provisional character, subject to permanent extension and updating. ${ }^{161}$ The Index was also understood as an itinerary of reading and an invitation to rewriting; open to all Catholic and erudite citizens willing to participate in the great enterprise of purification of bookish materials. ${ }^{162}$ The Reglas, mandatos $y$ advertencias generales opening the Index's editions are a good sample of these conceptions. ${ }^{163}$

By the end of the eighteenth century, the bull Sollicita ac provida (1753) by Pope Benedict XIV, which headed the Roman Index of 1758 and influenced

158 I have examined this practice in detail in: Beck Varela (2013) 325-348.

159 Defourneaux (1963).

160 See Hespanha (2007) 55-66, criticizing the anachronistic approach of many historians, when they project onto the early modern legal experience the contemporaneous views on law.

161 Savelli mentions the frequent lists of updates for new prescriptions (SAVELLI [2011] 30).

162 For the Portuguese context, see the remark of Payan Martins: «Proibir não é simplesmente dar execução ao índice em vigor; é um procedimento, muito mais complexo, de permanente superação das omissões e desactualização do próprio Index; é o exercício de um poder onde as lacunas legislativas obrigam os agentes censórios a regerem-se por critérios subjectivos, condicionados, não raro, pela conjuntura política, social e económica, ainda que, internamente, vigore o príncipio de pautarem a sua actuação pelas regras seguidas em Roma ou em Espanha» (Martins [2005] 135).

163 The fourth warning, incorporated later, stated that: «[...] Y esta advertencia puede servir para los que, menos atentos que deben ser, se querellan de que no se permiten todos los libros de los herejes, que no son de argumento herético, ni contienen herejía. A los cuales se responde que se permiten muchos; los que se prohiben es, ó porque no han llegado a nuestras manos, ó porque no consta de la utilidad: y aunque tal vez conste, no es bien permitirlos a todos sugetos, y por observar el estilo de la Iglesia, que en pena de su delito no permite que corran y se lean aun aquellos libros que no contienen herejía. Pero ordenamos y mandamos que todas las veces que alguna persona erudita y piadosa presentase algun libro de estos en el Consejo ó en sus tribunales para que le remitan á él, visto, examinado y censurado, puesta la nota de autor condenado y obra permitida, constando que es ó puede ser de utilidad, se le permitirá, y no de otra manera» (in Index [1707], s. p.; Índice [1790] xxxiii). 
the Royal Decree (Real Cédula) of 16 June 1768 in Spain, reinforced the practice of hand expurgation by the readers themselves, without the intermediation of the Inquisitional authorities. ${ }^{\mathbf{1 6 4}}$

This lack of uniformity brings us again to the locality and the diversity, even within the bounds of what we today identify as the religious borders in early modern Europe. Significant differences in the access to legal books and in reading practices are one of the many elements that impede us from perceiving Catholic Europe as a space of intellectual or religious unity. ${ }^{\mathbf{1 6 5}}$ Alien to the hypothetical intentions and merits of a dead author or of a remote biblical authority, the meaning and reliability of a flexible text, the commentaries on the Institutes of Justinian, the fruit of a collaborative, plural, uncertain author, depended essentially on the activity of various Catholic readers, who adapted his plural readings also to the commandments of a vacillating, uncertain authority.

\section{Epilogue}

Never before had the impression been so strong, that our knowledge has "lost" its temporal and spatial constraints as in today's era of technology. Besides enabling immediate communication, computer technology has multiplied ad infinitum our possibilities of storing, selecting, and accessing knowledge, and transcending physical restrictions. Tools like Google have not only transformed our way of accessing and diffusing information, but are apparently affecting our mental focus and our way of creating knowledge

164 This Real Cédula of 1768, invoking the papal bull, determined, despite the opposition of the Inquisitor-General, that the owners themselves of books could expurgate the passages: «2 [...] Conviene tambien se determine, en los que se han de expurgar, desde luego los parages ó folios, porque de este modo queda su lectura corriente, y lo censurado puede expurgarse por el mismo dueño del libro; advirtiéndose así en el edicto, como quando la Inquisicion condena proposiciones determinadas» (Novísima Recopilación, 8, 18, 3). See the dispute, concerning these regulations, between the prosecutors of the Council of Castile and the inquisitor general Quintano Bonifaz, in the document titled Consulta del Consejo y resolución de Su Majestad a súplica del Inquisidor general sobre las reglas dadas por S. M. para la expurgación y probibición de los libros, of 28 February 1769 (Manuscript of the Spanish National Library, nr. 10863).

165 MCKitTerick (2003) 163. For the differences in censorship practices and criteria within the Catholic space: Savelli (2011) 9; Beck Varela (2013) 292-300. 
as well. ${ }^{166}$ «Never has a communications system played so many roles in our lives ... as the Internet does today», synthetizes Carr; «The Internet [...] is subsuming most of our other intellectual technologies. It's becoming our map and our clock, our printing press and our typewriter, our calculator and our telephone, and our radio and our TV». ${ }^{\mathbf{1 6 7}}$ Among the practitioners of law, there is even a growing impression that the medium itself is responsible for a deterioration of the quality of legal sources, such as judges' sentences or doctrine. ${ }^{168}$

The legal historians gathered together in this volume represent probably the last generation which has been educated under the predominance of the printed text and has lived, especially in the past two decades, the increasing concurrence of online tools. All of us have begun our university careers handling manual catalogues and paper cards; many of us have travelled at least once to consult a rare manuscript or printed source in a remote archive or library, and some have even typed their first academic writings on a memorable Olivetti.

Readers in the age of the manual printing press had the same impression that their knowledge could transcend their immediate temporal and spatial dimensions, although not in the stable way we tend now to conceive. We have seen some clues of how it operated in practice, and how it affected the creation and diffusion of legal knowledge in the early modern centuries. Their notion of text was possibly as uncertain, as collaborative, as collective, as the one lying under the practices of Wikipedia users. Their medium had facilitated the possibility of plagiarism and free appropriation of texts as intensively as ours have. They depended on the reader, as we do, to construct text credibility; the conception of the printed text as something fixed, perfect and constant, the one with which this generation has started its instruction, is the outcome of a long historical development. As educators in law schools, we face as well the daily challenge to offer our students solid criteria to select among the infinitude of information provided by Google, or to learn how to

166 «When I mention my troubles with reading to friends and acquaintances - literary types, most of them - many say they're having similar experiences. The more they use the Web, the more they have to fight to stay focused on long pieces of writing». CARR (2008).

167 CARr (2008).

168 Judith Martins-Costa draws a desolating picture of the copy-and-paste of opinions in the daily life of legal professionals in Brazil (Martins-Costa [2014]). 
attribute authorship when constructing a legal reasoning. For the contemporary generation, as never before, the "physical" space occupied by knowledge seems unlimited, and the time for accessing it, minimal.

This brief and partial overview on the ways law was conceived, created and diffused in the early modern centuries leads us to historicize our discipline, its material and human conditions. This itinerary, whose intention was somehow to enhance our awareness of the spatial and temporal dimensions of legal knowledge in the past, might open a window to understand how these dimensions are being reshaped today and might be redesigned in the future.

\section{Bibliography}

Primary sources

Manuscript:

Consulta del Consejo y resolución de Su Majestad a súplica del Inquisidor general sobre las reglas dadas por S. M. para la expurgación y prohibición de los libros, 28 February 1769, Biblioteca Nacional de España (nr. 10863)

\section{Printed:}

Anisson (1702), Bibliographia Anissoniana, seu Catalogus Librorum qui prostant in aedibus Sociorum Anisson, Possuel et Rigaud, tam Parisiis quam Lugduni, ad annum 1702, Lugduni: Anisson

Brückner [Präes.], Wilhelm Hieronymus, Johan Caspar Brocris [Resp.] (1717), De plagio literario in studio juris, Jenae: Litteris Mullerianis

Camus, Armand-Gaston, Andre Dupin (1832), Bibliothèque choisie des livres de droit qu'il est le plus utile d'acquérir et de connaître. $5^{\mathrm{a}}$ ed. aum. A. Dupin, II, Paris: Gobelet-Warée

Cunha, Jerónimo da (1743), Perfectus advocatus, hoc est tractatus de patronis, sive advocatis, theologicus, juridicus, historicus, et poeticus [...] Ulyssipone: ex typis Joannis Baptiste Lerzo, 1743

Gundling, Nicolaus Hieronymus (1707), Otia deren III. Auflage von vielen andern solle begleitet werden. Nebst einem vollständigem Register über alle 3. Theile, Franckfurt und Leipzig: zufinden in der Regnerischem Buchhandlung

Ianuario, Iosephi Aureli de (1733) [Gennaro, Giuseppe Aurelio de], Respublica iurisconsultorum. Editio altera, ad exemplar neapolitanum expressa accedit Friderici Ottonis Menckenii, Lipsiensis, ad autorem epistola, Lipsiae: apud Iacobum Schusterum 
INDEX (1707), Novissimus librorum prohibitorum et expurgandorum Index pro Catholicis Hispaniarum Regnis Philippi V. Regis Catholici [Ab Exmo. Dno. D. Didaco Sarmiento et Valladares inceptus, et ab Illmo. Dno. D. Vitale Marin, perfectus...] ..., Matriti: ex typographia Musicae

INDEX (1790), Indice ultimo de los libros prohibidos y mandados expurgar: para todos los reynos y señoríos del catolico rey de las Españas, el señor Don Carlos IV, contiene en resumen todos los libros puestos en el Indice Expurgatorio del año 1747, y en los Edictos posteriores, asta fin de diciembre de 1789. Formado y arreglado con toda claridad y diligencia, por mandato del Excmo. Sr. D. Agustin Rubin de Cevallos, Inquisidor General, y Señores del Supremo Consejo de la Santa General Inquisicion: impreso de su orden, con arreglo al exemplar visto y aprobado por dicho Supremo Consejo, Madrid: en la Imprenta de Don Antonio Sancha

Lipenius, Martinus (1757), Bibliotheca Realis Juridica post Virorum Clarissimorum Friderici Gottlieb Struvii et Gottlob Augusti Jenichenii Curas Emendata. T. II, Leipizig [Facsimile edition: Hildesheim / New York: Georg Olms Verlag, 1970] Ludewig, Johann Peter von (1731), Vita Iustiniani M. atque Theodorae, Augustorum nec non Triboniani, iurisprudentiae iustinianae proscenium. Fide coaeuorum, Latii et Graeciae, scriptorum; numismatum; conciliorum; legum; litterarum; codicillorum; lapidum; picturarum; musiuorum aliorumque monumentorum, cum ad legum et corporis iuris intelligendam historiam, tum ad noui iuris architectorum apologiam; dispulsis autorum, in vario scientiarum genere, erroribus et calumniis. Halae Salicae: Impensis Orphanotrophei

Meermann, Gerard (1751-1753), Novus thesaurus juris civilis et canonici, continens varia et rarissima optimorum interpretum, imprimis Hispanorum et Gallorum opera, tam edita antehac, quam inedita, 7 vol., Hagae Comitum

Morhof, Daniel Georg(1708), Polyhistor, in tres tomos, literarium ... philosophicum et practicum ... opus posthumum ..., T. III. Lubecae: Sumptibus Petri Böckmanni

Mynsinger von Frundeck, Joachim (1691), Mynsingeri a Frundeck auctoris damnati Apotelesma, id est, corpus perfectum scholiorum ad Institutiones Iustinianeas pertinentium. Praeter jus novissimum tam ex Novellis constitutionibus, quam Imperialibus recessibus et observationibus Cam. Imp. fundamenta quoque, et leges allegatas, pluribus in locis castigatas, restitutas et distinctas, de voluntate et consilio auctoris, ex recognitione Arnoldi de Reyger, IC. Accesserunt nunc demum, paragraphorum summae, Jurisconsultorum clarissimorum notae, graduum descriptiones; et librorum titulorumque synopses; cum remissionibus ad ipsius Mynsinger, Gailli observationes Imp. Cam. Studio et labore Ioannis Fehi, IC. Editio reformata, et Indici Sacrae Inquisitionis expurgatorio conformis facta, Lugduni: sumpt. Anissoniorum, Ioan. Posuel \& Claudii Rigaud [...]

Nettelbladt, Daniel (1757), Nachricht von des Arnold Vinnius Leben und Schriften, in: Hallische Beyträge zu der juristischen Gelehrten Historie. 2. Band, 5. 
bis 8. Stück, Halle im Magdeburgischen, Rengerischen Buchhandlung [8. Stück], 638-680

Nettelbladt, Daniel (1764), Initia historiae litterariae iuridicae universalis, Halae Magdeburgicae, prostat in Officina libraria Rengeriana

Nettelbladt, Daniel (1781), Systema elementare doctrinarum propaedeuticarum iurisprudentiae positivae Germanorum communis. Praemissae sunt praecognitorum eruditionis generalium primae lineae, Halae: in Officina Rengeriana

Pütter, Johann Stephan (1783), Litteratur des teutschen Staatsrechts, vom geheimen Justizrath Pütter zu Göttingen. Dritter und letzter Theil, Göttingen: im Verlag der Wittme Bandenhoeck [Facsimile edition: Frankfurt am Main: Antiquariat Sauer \& Auvermann 1965]

Sala y Bañuls, Juan (c. 1786), Additiones et emendationes ad primam editionem operis Vinnius castigatus, jus Hispanum praecipue respicientes, quae in secunda suis interiiciuntur locis, hic seorsum editae in gratiam eorum qui utuntur prima, s. l., s.n., s. a.

Sempere y Guarinos, JuAn (1785), Ensayo de una biblioteca española de los mejores escritores del reynado de Carlos III, T. III, Madrid: Imprenta Real [facsimile edition Madrid: Gredos, 1969]

Schwartz, Johann Conrad [Präes.], Wilhelm Tobias Stempel [Resp.] (1701), Tentaminis de plagio literario ..., Hallae Magdeburgicae: litteris Christiani Henckelii, Acad. Typogr

Struve, Burkhard Gotthelf (1756), Bibliotheca iuris selecta. Acessit Bibliotheca selectissima iuris studiosorum [...] emendavit et copiose locupletavit Christian Gottlieb Buder. Editio octava, Ienae: apud Christian. Henr. Cuno [facsimile edition: Aalen, Scientia Verlag, 1970]

Taisand, Pierre (1737), Les vies des plus célèbres jurisconsultes, de toutes les nations, tant anciens que modernes, savoir, latins ou romains, françois, espagnols, italiens, allemans, anglois, hollandois, \&c. Tirées des meilleurs auteurs qui en ont écrit; et mises en leur jour par ordre alphabétique. Nouvelle édition, augmentée d'un tiers, Paris, Quai de Gesvres

Terrasson, Antoine (1750), Histoire de la jurisprudence romaine, contenant son origine et ses progrès depuis la fondation de Rome jusqu'à présent ... L'énumération des editions du corps de droit civil: les vies et le catalogue des ouvrages des jurisconsultes, tant anciens que modernes: avec un recueil de ce qui nous reste de contrats, testamens, et autres actes judiciaires des anciens Romains. Pour servir d'Introduction à l'étude du Corps de Droit Civil, à la lecture des commentateurs du Droit Romain, et à l'ouvrage intitulé les Loix Civiles dans leur ordre naturel, Paris: Chez B. Brunet, Imprimeur-Libraire, rue Saint Jacques, vis-à-vis celles des Mathurins

Tномаsius, Јаков (1673), Dissertatio philosophica de plagio literario ..., Lipsiae: sumpt. Christoph Enoch

Тномаsıus, Јаков (1679), Ad Disputationem M. Jacobi Thomasii de plagio literario accessiones ..., Jenae: Litteris Joh. Jacob. Bauhoferi 
Torres y Velasco, Antonio (1735), Institutiones Hispanae practico-theoricae commentatae ..., Matriti: Apud Haeredes Joannis Garcia Infanzon

Vinnius, Arnoldus (1723-1724), Arnoldi Vinnii, J.C., authoris damnati, opus vero cum expurgatione permissum, in quatuor libros Institutionum imperialium commentarius academicus et forensis [...] Correcta atque emendata secundum Indicem expurgatorium Sanctissimae Inquisitionis Hispaniae, publicatum anno 1707, Matriti: Sumpt. Francisci Lasso, et Iosephi Lasso, filii. Ex officina regia, apud Iosephum Rodriguez de Escobar. Sanctae Cruciatae, \& Hispanicae Academiae Typographum

Vinnius, Arnoldus (1772), In quatuor libros Institutionum imperialium commentarius academicus et forensis. Jo. Gottl. Heineccius JC. recensuit, et praefationem notulasque adjecit, cui Jacobi Gothofredi J.C. Historia et bibliotheca juris civilis Romani praeponitur. Accedit etiam ad calcem Edictum perpetuum Salvii Juliani a Guilielmo Ranchino collectum. Editio novissima [...], Neapoli: apud Dominicum Terres

Vinnius, Arnoldus (1786), In quatuor libros Institutionum imperialium commentarius academicus et forensis. Editio nova, in usum Hispanae iuventutis adornata, in qua auctoris textus ad Batavicar. edd. fidem exhibetur, Graeca Latio donatur, patrii iuris fontes, tum ius ipsum et receptae de eo in praxi DD. Hispanorum opiniones singulis quibusque $\$ \mathbb{S}$. indicantur atque exponuntur [...], Valentiae: apud Benedictum Monfort Typog.

Vinnius, Arnoldus (1846), Comentario académico y forense del célebre jurisconsulto Arnoldo Vinnio á los cuatro libros de las Instituciones imperiales de Justiniano, anotado por el jurisconsulto J. Gottlieb Heineccio, y seguido de las cuestiones selectas del mismo autor. Traducción al Castellano, adicionada con las variantes del derecho español y las diferencias más notables del derecho municipal de Cataluña. Por el licenciado en jurisprudencia D.J. P. y B. Tomo I, Barcelona: Imprenta de José Torner

Wesenbeck, Matthäeus (1639), Commentaria quae olim Paratitla, in Pandectas iuris civilis et Codicis Iustiniani libros. Nunc demum, post numperam Academiam Duacensis emendationem, accuratius recognita, et quibusdam sive erroribus, sive offensivis dogmatibus ac doctrinis, quibus adhuc respersa erant, repurgata, geminoque indice locupletata, Antuerpiae: apud Hieronymum Verdussium

\section{Secondary sources}

Alonso Romero, María Paz (1997), Lectura de Juan Gutiérrez (c. 1535/1540-1618), un jurista formado en Salamanca, in: Initium 2, 447-484

Alvarado Planas, Javier (2007), Justicia, libertad y censura en la Edad Moderna, Madrid: BOE

Arnold, John, Martyn Lyons (2001) (ed.), A History of the Book in Australia, 1891-1945: A National Culture in a Colonised Market, Brisbane: University of Queensland Press 
Barbier, Frédéric, Catherine Bertho Lavenir (2003), Histoire des médias: de Diderot à internet. 3e ed. revue et complétée, Paris: Armand Colin

Barbier, Frédéric, István Monok (2009) (ed.), Cinquante ans d'histoire du livre, de «L'apparition du livre» (1958) à 2008: bilan et projets, Budapest: Országos Széchényi Könyvtár

Barrientos Grandón, Javier (1992), La cultura jurídica en el Reino de Chile. Bibliotecas de Ministros de la Real Audiencia de Santiago (s. XVII-XVIII), Santiago (Chile): Escuela de Derecho Universidad Diego Portales

Barthes, Roland (1968), La muerte del autor, in: El susurro del lenguaje. Más allá de la palabra y la escritura, (trad. C. Fernández Medrano), Barcelona: Paidós [1987], 65-71

Beardsley Jr., Theodore S. (1986), The Classics in Spain: the Sixteenth versus the Seventeenth Century, in: Faulhaber, Charles et al. (eds.), Studies in Honor of Gustavo Correa. Potomac, Maryland: Scripta Humanistica 17, 11-27

Beck Varela, Laura (2013), Literatura Jurídica y Censura. Fortuna de Vinnius en España, Valencia: Tirant lo Blanch

Bell, Bill et al. (2007-2011) (ed.), The Edinburgh History of the Book in Scotland, Edinburgh: Edinburgh University Press, 4 vols.

Beneduce, Pasquale (1994), Traduttore - traditore. Das französische Zivilrecht in Italien in den Handbüchern der Rechtswissenschaft und -praxis, in: ScHulze, R. (ed.), Französisches Zivilrecht in Europa während des 19. Jahrhunderts, Berlin: Duncker \& Humboldt, 205-241

Biagioli, Mario (1993), Galileo, Courtier: The Practice of Science in the Culture of Absolutism, Chicago: University of Chicago Press

Birocchi, Italo (2006), La formazione dei diritti patri nell'Europa moderna tra politica dei sovrani e pensiero giuspolitico, prassi ed insegnamento, in: Birocchi, Italo, Antonello Mattone (ed.), Il diritto patrio tra diritto comune e codificazione (secoli XVI-XIX), Roma: Viella, 17-71

Blair, ANn (2010), Too much to know: managing scholarly information before the Modern Age, New Haven: Yale University Press

Botrel, Jean-François et al. (2003) (ed.), Historia de la edición y de la lectura en España, 1475-1914, Madrid: Fundación Germán Sánchez Ruipérez

Bourdieu, Pierre (1984), Homo academicus, Paris: Les Éditions du Minuit

Bouza Álvarez, Fernando (1999), Comunicación, conocimiento y memoria en la España de los siglos XVI y XVII, Salamanca: Publicaciones del SEMYR

Bouza Álvarez, Fernando (2001), Corre Manuscrito: una historia cultural del Siglo de Oro, Madrid: Marcial Pons

Brokaw, Cinthia, Peter Kornick (2013) (ed.), The history of the book in East Asia, Farnham: Ashgate

Buigues, Jean-Marc (2003a), La sociedad de los autores, in: Botrel, Jean-François et al. (ed.), Historia de la edición y de la lectura en España, 1475-1914, Madrid: Fundación Germán Sánchez Ruipérez, 292-301

Buigues, JeAn-Marc (2003b), Las materias: tradición y modernización, in: Botrel, JEAN-François et al. (2003), 317-328 
Buigues, Jean-Marc (2003c), Los lectores: oficios, profesiones, estados, in: Botrel, JeAn-François et al. (2003), 424-431

Cappellini, Paolo, Bernardo Sordi (2002) (ed.), Codici: una riflessione di fine millennio (atti dell'incontro di studio: Firenze, 26-28 ottobre 2000), Milano: Giuffrè

Carr, Nicholas (2008), Is Google Making Us Stupid? What the internet is doing to our brains, in: The Atlantic, 1, July

Cátedra, Pedro, Anastasio Rojo (2004), Bibliotecas y Lecturas de Mujeres. Siglo XVI, Madrid: Fundación Germán Sánchez-Ruipérez

Cátedra, Pedro, María Luisa López-Vidriero (2004) (ed.), La memoria de los libros: estudios sobre la historia del escrito y de la lectura en Europa y América, Salamanca: Instituto de Historia del Libro y de la Lectura

Cavallo, Guglielmo, Chartier, Roger (1998) (ed.), Historia de la lectura en el mundo occidental, Madrid: Taurus

Chartier, Roger (1981), L’Ancien Régime Typographique. Réflexions sur quelques travaux récents, in: Annales E.S.C. 36, 191-209

Chartier, Roger (1985), Du livre au lire, in: Chartier, Roger (ed.), Pratiques de lecture. Marseille: Rivages, 62-88

Chartier, Roger, Henri-Jean Martin (1989-1991) (eds.), Histoire de l'édition française, 4 vol., Paris: Fayard

Chartier, Roger (1991), The Cultural Origins of the French Revolution, Durham: Duke University Press

Chartier, Roger (1992), Laborers and Voyagers: from the text to the reader, in: Diacritics (Summer 1992) 22:2, 49-61

Chartier, Roger (1994), Figuras del autor, in: El orden de los libros: lectores, autores, bibliotecas en Europa entre los siglos XIV y XVIII, Barcelona: ed. Gedisa, 41-67

Chartier, Roger (1995), De l'histoire du livre à l'histoire de la lecture: les trajectoires françaises, in: BöDEKer, Hans ERICH (ed.), Histoires du livre. Nouvelles orientations (actes du colloque du 6-7 septembre 1990), Paris: Maison des sciences de l'homme - IMEC éditions, 23-45

Chartier, Roger (2000), Entre poder y placer. Cultura escrita y literatura en la Edad Moderna, Madrid: Ediciones Cátedra

Clavero, Bartolomé (1991), Antidora. Antropología Católica de la Economía Moderna, Milano: Giuffrè

Clavero, Bartolomé (1992), Estudio introductorio, in: Jean Louis de Lolme, Constitución de Inglaterra, Madrid: Centro de Estudios Constitucionales, 9-21

Clavero, Bartolomé (1993), Presentación, in: Franckenau, Gerardo Ernesto de, Sagrados misterios de la justicia hispana [obra atribuida a Juan Lucas Cortés] (trad. María Ángeles Durán Ramas), Madrid: Centro de Estudios Constitucionales, 9-21

Cohen, Lara Langer, Jordan Alexander Stein (2012) (eds.), Early African American Print Culture, Philadelphia: University of Pennsylvania Press 
Colli, Vincenzo (2002) (ed.), Juristische Buchproduktion im Mittelalter, Frankfurt am Main: Vittorio Klostermann

Colli, Vincenzo (2005) (ed.), Giuristi medievali e produzione libraria: Manoscritti, autografi, edizioni, Stockstadt am Main: Keip Verlag

Collini, Stefan et al. (2014), What is Intellectual History?, in: History Today [3]: 35: 10 [4] (available in: http://www.historytoday.com/stefan-collini/whatintellectual-history)

Conde Naranjo, Esteban (2006), El Argos de la Monarquía. La policía del libro en la España ilustrada (1750-1834), Madrid: Centro de Estudios Constitucionales

Conde Naranjo, Esteban (1998), Medioevo Ilustrado. La edición erudita del Ordenamiento de Alcalá (1774), Sevilla: Universidad de Sevilla

Darnton, Robert (1979), The Business of Enlightenment: A publishing history of the Encyclopédie 1775- 1800, Cambridge: The Belknap Press de Harvard University Press

Darnton, Robert (1982), What is the history of books?, in: Daedalus, 111 (3), $65-83$

Darnton, Robert (1990), First Steps Toward a History of Reading, in: Darnton, Robert, The Kiss of Lamourette. Reflections in Cultural History, New York: Norton, 154-185

Darnton, Robert (1993), 'La France, ton café fout le camp!' De l'histoire du livre à l'histoire de la communication, in: A.R.S.S. 100: 1, 16-26 (available at: http://dx.doi.org/10.3406/arss.1993.3068)

Darnton, Robert (1995), The Forbidden Bestsellers of Pre-Revolutionary France, New York: Norton

Defourneaux, Marcelin (1963), L'Inquisition espagnole et les livres français au XVIII $^{\mathrm{e}}$ siècle, Paris: Presses Universitaires de France

De Los Reyes Gómez, Fermín (2000), El libro en España y América. Legislación y censura (siglos XV-XVIII), 2 vol., Madrid: Arco Libros

Detering, Heinrich (2002), Autorschaft: Positionen und Revisionen, Stuttgart: Metzler

Duve, Thomas (2012), Von der Europäischen Rechtsgeschichte zu einer Rechtsgeschichte Europas in globalhistorischer Perspektive, in: Rechtsgeschichte, 20, $18-71$

Eisenstein, Elizabeth L. (1997), The Printing Press as an Agent of Change: Communications and Cultural Transformations in Early-Modern Europe. Cambridge: Cambridge University Press

Eliot, Simon et al. (2007) (ed.), Introduction, in: Literary cultures and the material book, London: British Library

Enciso Recio, Luis Miguel (2002), Barroco e Ilustración en las bibliotecas privadas españolas del siglo XVIII, Discurso, Real Academia de Historia, Madrid: $\mathrm{RAH} /$ Elece

Feest, Johannes, David Nelken (2001) (ed.), Adapting legal cultures, Oxford: Hart Fögen, Marie-Therès, Günter Teubner (2005), Rechtstransfer, in: Rechtsgeschichte 7, $38-45$ 
Foucault, Michel (1969), Qué es un autor?, in: Obras esenciales. Entre filosofía y literatura, I (trad. Miguel Morey), Barcelona: Paidós [1999], 331-359

Frajese, Vittorio (2000), Le licenze di lettura e la politica del Sant'Uffizio dopo l'Indice clementino, in: L'Inquisizione e gli storici: un cantiere aperto (atti dei convegni lincei, 162), Roma: Accademia Nazionale dei Lincei, 179-220

Frajese, Vittorio (2006), Nascita dell'Indice. La censura ecclesiastica dal Rinascimento alla Controriforma, Brescia: Morcelliana

Gacto Fernández, Enrique (1992), Censura política e Inquisición. La Historia Pontifical de Gonzalo de Illescas, in: Revista de la Inquisición 2, 23-40

García Martín, Javier (2003), El juzgado de imprentas y la utilidad pública. Cuerpo y alma de una Monarquía vicarial, Bilbao: Universidad del País Vasco

Garriga, Carlos (2004), Estudio preliminar, in: Salgado Correa, Alejo, Libro nombrado Regimiento de Juezes [Sevilla, 1556], Madrid: Consejo General del Poder Judicial

Garrisson, Janine (1992), Leçon inaugurale in: SAUzet, Robert (ed.), Les frontières religieuses en Europe, Paris: Vrin, 9-13

Giaro, Tomasz (1995), Römisches Recht, Romanistik und Rechtsraum Europa, in: Ius Commune 22, 1-16

Gierl, Martin (2001), Kompilation und die Produktion vom Wissen im 18. Jahrhundert, in: Mulsow, Martin, Helmut Zedelmaier (ed), Die Praktiken der Gelehrsamkeit in der Frühen Neuzeit, Tübingen: Niemeyer, 63-94 (available at: http://dx.doi.org/10.1515/9783110936285.63)

Glendinning, Nigel (1983), Historia de la literatura española, 4. El siglo XVIII, $4^{\text {a }}$ ed., Barcelona: Ariel

Gordon, Daniel (1998), The great Enlightenment massacre, in: Mason, Haydn T. (ed.), The Darnton Debate. Books and Revolution in the eighteenth century [Studies on Voltaire and the Eighteenth Century, 359], Oxford: Voltaire Foundation, 129-156

Greenspan, Ezra, Jonathan Rose (1998), An introduction to Book History, in: Book History 1: 1, 3, ix-xi (available at: http://dx.doi.org/10.1353/ bh.1998.0005)

Gumbrecht, Hans Ulrich (1989), Conseguenze dell'estetica della ricezione o la teoria letteraria come sociología della comunicazione, in: Holub, Robert C. (ed.), Teoria della ricezione, Torino: Giulio Einaudi, 155-184

Hall, David (2007-2009) (ed.), A History of the Book in America, Chapel Hill: University of North Carolina Press, 5 vols.

Hallewell, Laurence (2005), O livro no Brasil: sua história, São Paulo: EdUSP

Hammond, Mary, Shafquat Towheed (2007) (ed.), Publishing in the First World War: Essays in Book History, London: Palgrave Macmillan

Hellinga, Lotte (1995), Planning the History of the Book in Britain: Seeking Definitions, in: Bödeker, Hans Erich (ed.) (1995), Histoires du livre. Nouvelles orientations (actes du colloque du 6-7 septembre 1990), Paris: Maison des sciences de l'homme - IMEC éditions, 61-71 
Helmrath, Johannes (2002), Diffusion des Humanismus. Zur Einführung, in: Helmrath, Johannes et al. (ed.), Diffusion des Humanismus. Studien zur nationalen Geschichtsschreibung europäischer Humanisten, Göttingen: Wallstein, 9-29

Hespanha, António Manuel (1990), Una historia de textos, in: Tomás y Valiente, Francisco et al. (ed.), Sexo barroco y otras transgresiones premodernas, Madrid: Alianza, 187-196

Hespanha, António Manuel (1993), Representación dogmática y proyectos de poder, in: Hespanha, António Manuel, La Gracia del Derecho. Economía de la cultura en la Edad Moderna (Transl. Ana Cañellas Haurie), Madrid: Centro de Estudios Constitucionales, 61-83

Hespanha, António Manuel (2007), Depois do Leviathan, in: Almanack Braziliense, 5 (maio), 55-66 (available at: http://dx.doi.org/10.11606/issn.1808-8139. v0i5p55-66)

Hespanha, António Manuel (2008), Form and Content in Early Modern Lawyers' Books: Bridging Material Bibliography with History of Legal Thought, in: Rechtsgeschichte, 12, 12-50

Hesse, Carla (1991), Enlightenment epistemology and the Laws of Authorship in Revolutionary France, 1777-1793, in: Law and the Order of Culture, Berkeley: University of California Press, 109-137

Infelise, Mario (1989), L'editoria veneziana nel' 700, Milano: Franco Angeli

Irwin, William (2002), The Death and Ressurrection of the Author?, Westport (Connecticut): Greenwood Press

Jack, Belinda (2012), The woman reader, New Haven: Yale University Press

Jannidis, Fotis et al. (1999), Rede über den Autor an die Gebildeten unter seinen Verächtern. Historische Modelle und systematische Perspektiven, in: JANNIDIS, Fotıs et al., Rückkehr des Autors. Zur Erneuerung eines umstrittenen Begriffs, Tübingen: Max Niemeyer, 3-35 (available at: http://dx.doi.org/ 10.1515/9783110944754.3)

Jaszi, Peter (1994), On the Author Effect: Contemporary Copyright and Collective Creativity, in: Woodmansee, Martha, Peter Jaszi (ed.), The Construction of Authorship. Textual appropriation in Law and Literature, Durham: Duke University Press, 29-56

Jaumann, Herbert (2000), Öffentlichkeit und Verlegenheit. Frühe Spuren eines Konzepts öffentlicher Kritik in der Theorie des 'plagium extrajudiciale' von Jakob Thomasius (1673), in: Scientia Poetica. Jahrbuch für Geschichte der Literatur und der Wissenschaften 4, 62-82

Jauss, Hans Robert (1989), La teoria della ricezione. Identificazione retrospettiva dei suoi antecedenti storici, in: Ноlub, Rовеrт C. (ed.), Teoria della ricezione, Torino: Giulio Einaudi, 3-26

Johns, Adrian (1998), The Nature of the Book. Print and Knowledge in the Making, Chicago: University of Chicago Press (available at: http://dx.doi.org/10.7208/ chicago/9780226401232.001.0001) 
Jostock, Ingeborg (2007), La censure négociée. Le contrôle du livre à Genève, 1560-1625, Généve: Droz

Juárez Medina, Antonio (1988), Las reediciones de obras de erudición de los siglos XVI y XVII durante el siglo XVIII español, Frankfurt: Peter Lang

Lewis, Charlton T., Charles Short (1879), A Latin Dictionary, founded on Andrews' edition of Freund's Latin dictionary, revised, enlarged, and in great part rewritten [...], Oxford: Clarendon Press

Llamosas, Esteban (2008), La literatura jurídica de Córdoba del Tucumán en el siglo XVIII. Bibliotecas corporativas y privadas. Libros ausentes. Libros prohibidos, Córdoba: Universidad Nacional de Córdoba

Marsá, María (2004), Los libros de Derecho en la imprenta vallisoletana de los siglos XVI y XVII, in: Cátedra, Pedro, María Luisa López-Vidriero (2004) (ed.), La memoria de los libros: estudios sobre la historia del escrito y de la lectura en Europa y América, Salamanca: Instituto de Historia del Libro y de la Lectura, I, 341-354

Martin, Henri-Jean (1969), Livre, pouvoirs et société à Paris au XVII ${ }^{\mathrm{e}}$ siècle (1598-1701), Genève: Droz, 1984 [réimpr. de l'éd. de Genève, 1969]

Martín Abad, Julián (2003), La edición española fuera de España, in: Botrel, JeanFrançois et al. (2003), 105-111

Martínez de Bujanda, Jesús (1984-2002), Index des livres interdits, 11 vol., Genève: Centre d'études de la Renaissance

Martínez Martín, Jesús (2000), Editores, libreros y público en Madrid durante la II República, Madrid: Artes Gráf. Municipales, Área de Régimen Interior y Patrimonio

Martínez Martín, Jesús (2001) (ed.), Historia de la edición en España (1836-1936), Madrid: Marcial Pons

Martínez Rus, Ana (2005), La lectura pública durante la Segunda República, in: Ayer 58, 179-203

Martins, Maria Teresa Esteves Payan (2005), A censura literária em Portugal nos séculos XVII e XVIII, Coimbra: Fundação Calouste Gulbekian

Martins-Costa, Judith (2014) (ed.), Modelos de direito privado, São Paulo: Marcial Pons

Mazzarella, Ferdinando (2010), Diritto e invenzioni. Un'introduzione storica, in: Rivista di storia del diritto italiano 83, 69-138

McKenzie, Donald F. (1977), Typography and Meaning: The Case of William Congreve, in: Barber, Giles, Bernhard Fabian (eds.), Buch und Buchhandel in Europa im achtzehnten Jahrhundert, Hamburg: E. Hausdewell, 81-125

McKenzie, Donald F. (1991), La bibliographie et la sociologie des textes, Paris: Éditions du Cercle de la Librairie

McKitterick, David (2003), Print, Manuscript and the Search for Order, 1450-1830, Cambridge: Cambridge University Press

Minnis, Alastair J. (1984), Medieval theory of authorship. Scholastic literary attitudes in the later Middle Ages, London: Scolar Press 
Moll, Jaime (2003), El impresor, el editor y el librero, in: Botrel, J.-F. et alii (eds.), Historia de la edición y de la lectura en España (1472-1914). Madrid: Fundación Germán Sánchez Ruipérez, 77-82

Morgan, Nigel et al. (1999-2012) (ed.), The Cambridge history of the book in Britain, 6 vol., Cambridge: Cambridge University Press

Mulsow, Martin (2006), Practices of Unmasking: Polyhistors, Correspondence, and the Birth of Dictionaries of Pseudonimity in Seventeenth-Century Germany, Journal of the History of Ideas 67: 2 (April), 219-250

Mulsow, Martin, Helmut Zedelmaier (2001) (ed.), Die Praktiken der Gelehrsamkeit in der Frühen Neuzeit, Tübingen: Niemeyer

Muñoz de Bustillo, Carmen (2006), Estudio introductorio, in: Gutiérrez, Juan, Fueros vascos: fundamentos de derecho (1593) (trad. María de los Ángeles Durán Ramas), Madrid: Centro de Estudios Políticos y Constitucionales

Orsini, Francesca (2013) (ed.), The history of the book in South Asia, Farnham: Ashgate

Osler, Douglas J. (1995), Text and Technology, in: Rechtshistorisches Journal 14, 309-331

Osler, Douglas J. (1997), The Myth of European Legal History, in: Rechtshistorisches Journal 16, 393-410

Osler, Douglas J. (2009), Jurisprudence of the Baroque. A Census of Seventeenth Century Italian Legal Imprints, 3 vols., Frankfurt am Main: Vittorio Klostermann

Pastore, Alessandro (2007), Introduzione, in: Pastore, Alessandro (ed.), Confini e frontiere nell'età moderna: un confronto fra discipline, Milano: Franco Angeli, 7-20

Péligry, Christian (1990), Le marché espagnol, in: Chartier, Roger, Henri-Jean Martin (ed.), Histoire de l'édition française, II. Le livre triomphant, 1660-1830, Paris: Fayard, 481-492

Peset, Mariano (1975), Estudio preliminar, in: Gregorio Mayáns y Siscar, Epistolario, IV. Mayáns y Nebot (1735-1742). Un jurista teórico y un práctico, Valencia: Ayuntamiento de Oliva

Pettegree, Andrew, Matthew Hall (2004) The Reformation and the Book: a Reconsideration, in: Historical Journal 47, 785-808 (available at: http:// dx.doi.org/10.1017/S0018246X04003991)

Pethers, Matthew (2012), Online Roundtable: A History of the Book in America, in: Journal of American Studies, 46: 2, 1-2 (available at: http://dx.doi.org/ $10.1017 /$ S0021875812000497)

Price, Leah (2004), Reading: The State of the Discipline, in: Book history 7, 303-320 (available at: http://dx.doi.org/10.1353/bh.2004.0023)

Ranieri, Filippo (1982), Juristische Literatur aus dem Ancien Régime und historische Literatursoziologie. Eine methodologische Vorüberlegungen, in: Aspekte europäischer Rechtsgeschichte. Festgabe für Helmut Coing, Frankfurt am Main: Vittorio Klostermann, 293-321 
Renouard, Philippe (1995), Jean Anisson, in: Répertoire des imprimeurs parisiens: libraires et fondeurs de caractères en exercice à Paris au XVII ${ }^{\mathrm{e}}$ siècle, Nogent Le Roi: Librairie des Arts et Métiers-Éditions

Repgen, Tilmann (2001), Daniel Nettelbladt, in: Michael Stolleis (ed.), Juristen. Ein biographisches Lexikon, München: Beck, 467-468

Rial Costas, Benito (2013) (ed.), Print culture and peripheries in Early Modern Europe: a contribution to the history of printing and the book trade in small European and Spanish cities, Leiden: Brill

Roberts, Jane et al. (2010) (ed.), The History of the Book in the West, Farnham: Ashgate

Roper, Geoffrey (2013) (ed.), The history of the book in the Middle East, Farnham: Ashgate

Rose, Jonathan (2001), The Intellectual Life of the British Working Classes, New Haven: Yale University Press

Rose, Jonathan, Simon Eliot (2007) (ed.), A Companion to the History of the Book, Malden: Blackwell

Rose, Mark (1993), Authors and Owners. The Invention of Copyright, Cambridge: Harvard University Press

Ross, Marlon (1994), Authority and Authenticity: Scribbling Authors and the Genius of Print in Eighteenth-Century England, in: Woodmansee, Martha, Peter Jaszi (eds.), The Construction of Authorship. Textual appropriation in Law and Literature, Durham: Duke University Press, 231-257

SAntoro, Marco (2008), Storia del libro italiano: libro e società in Italia dal Quattrocento al nuovo millennio, Milano: Editrice Bibliografica

Shelley Rubin, Joan (2003), What is the History of the History of Books?, in: The Journal of American History 90 (September), 555-576

Savelli, Rodofo (2011), Censori e giuristi. Storie di libri, di idee e di costumi (secoli XVI-XVII), Milano: Giuffrè

Schiavone, Aldo (2005), Ius. L'invenzione del diritto in Occidente, Torino: Giulio Einaudi

Sher, Richard (2006), The Enlightenment and the Book. Scottish Authors and their Publishers in eighteenth-century Britain, Ireland, and America, Chicago: University of Chicago Press

Stempel, Wolf-Dieter (1989), Aspetti generici della ricezione, in: Holub, Robert C. (ed.), Teoria della ricezione, Torino: Giulio Einaudi, 185-200

Suarez, Michael F. et al. (2010) (ed.), The Oxford Companion to the Book, Oxford: OUP

Tomás y Valiente, Francisco (1988), El Pensamiento Jurídico, in: Artola, Miguel (ed.), Enciclopedia de historia de España, Vol. 3: Iglesia, Pensamiento, Cultura, Madrid: Alianza, 327-408

Torre Villar, Ernesto de la (2009), Breve Historia del Libro en México, México: Unam

Torremocha Hernández, Margarita (1993), La enseñanza entre el inmovilismo y las reformas ilustradas, Valladolid: Universidad de Valladolid 
Vallejo, Jesús (1996), El Fuero Real bajo las luces, o las sombras de la edición de 1781, in: Initium 1, 611-643

Vallejo, Jesús (1998), Academia y Fuero. Historia del Real en la Real de la Historia, in: Initium 3, 419-484

Vallejo, Jesús (2000), Estudio preliminar, in: Duque de Almodóvar, Constitución de Inglaterra, Madrid: Centro de Estudios Constitucionales

Villalta, Luiz Carlos (2013), A história do livro e da leitura no Brasil colonial: balanço historiográfico e proposição de uma pesquisa sobre o Romance, in: Campinas: IEL (available in: http://www.caminhosdoromance.iel.unicamp.br/ estudos/ensaios/livroeleitura.pdf)

Weinrich, Harald (1989), Per una storia letteraria del lettore, in: Holub, Robert C. (ed.), Teoria della ricezione, Torino: Giulio Einaudi, 27-41

WijfFels, Alain (1993), Law Books in Cambridge Libraries, 1500-1640, in: Transactions of the Cambridge Bibliographical Society 10: 3, 359-412

Woodmansee, Martha (1994), On the Author Effect: Recovering Collectivity, in: Woodmansee, Martha, Peter Jaszi (eds.), The Construction of Authorship. Textual appropriation in Law and Literature, Durham: Duke University Press, $15-28$

Zimmermann, Michael (2001) (ed.), Auctor et auctoritas. Invention et conformisme dans l'écriture médiévale, Paris: École de Chartes 

Floriana Colao

\section{Per una Storia del Processo Penale «all'Italiana». «Astratte Modellistiche» e «Abitudini Profondamente Radicate»}

\section{Premessa. I modelli e l'Italia}

«Le abitudini giudiziarie cadono fuori del tempo politico». ${ }^{1}$ L'indicazione di Franco Cordero ci è parsa riflettere una caratteristica peculiare del processo penale italiano: una sorta di assenza di dimensione storica nel suo essere eterno "grande malato», ${ }^{2}$ anche nel momento in cui, di recente, si è ragionato sul fecondo incontro tra storici e processualpenalisti. ${ }^{3}$ Ad esempio il tema dell'eccessiva durata dei procedimenti si è rivelato terreno d'elezione di una polemica senza dimensione storica nella storia europea moderna e contemporanea; ${ }^{4}$ così come, nel tempo, è stata costante la ricerca di modelli oltre il luogo teatro della giustizia italiana, modelli - tra tutti quello accusatorio - destinati ad avere senso come strumento di legittimazione di ideologie e tecniche elaborate localmente, dal legislatore nazionale, in un originale «Italian style». ${ }^{5}$

Tra Otto e Novecento sono stati i giuristi gli artefici della codificazione penalprocessuale, fin dal momento in cui Francesco Carrara evocava il «progredire della dottrina» per cambiare il codice del 1865, «indegno di un

1 Cordero (1997b) 233.

2 Per indicazioni sulle diagnosi e cure che dal secondo dopoguerra i giuristi proposero per il processo grande malato, e sul guardasigilli De Pietro, che nel 1955 varò un'ampia riforma del codice di procedura del 1930, con l'asserire «dalla malattia alla guarigione c'è la convalescenza» sia consentito di rinviare a Colao (2013b) 84 ss.

3 Cfr. tra gli altri Garlati (2010a) 1 ss.; Cappellini (2011) 3 ss.; Orlandi (2012a) 79 ss.; Seminara (2012) 85 ss.

4 Cfr. ora Cavina (2012a) 7 ss.

5 Pisani (1998). Sulla costruzione di una identità nazionale anche penale, da Beccaria all'Unità cfr. ora Pifferi (2013a) 275 ss. 
popolo che dicesi libero». ${ }^{6}$ Sono stati diversi i contesti: l'Italia liberale era teatro di un grande confronto, dottrinale e parlamentare, sui contenuti di un processo adeguato all'ordine liberale, con aperture ad un idealizzato modello anglosassone, nella «attesa» durata fino al $1913 .{ }^{7}$ Tra progetti e leggi delega ispirati all'accusatorio, sia pure «all'italiana» - come avrebbe ammesso Gian Domenico Pisapia, incaricato dal 1974 della redazione del codice varato nel $1988,{ }^{8}$ l'attesa si sarebbe ripetuta nell'Italia repubblicana. Era stata più semplice l'esperienza del codificatore Vincenzo Manzini, a suo dire artefice di un codice dalla continuità tecnica e discontinuità ideologica, «per la prima volta formato senza il consueto concorso di commissioni».?

Il «possente (benché discusso) paradigma legal-positivistico», ${ }^{\mathbf{1 0}}$ il diritto considerato sub specie legis, e dunque lo studio della codificazione penale e penalprocessuale, non può appagare la storia del processo, fatta di regole e di dottrine, ma anche di giurisprudenza, di politiche per disciplinare la penalità, di ideologie per legittimare il potere pubblico. Non a torto due recenti dense sintesi per l'Appendice Ottava dell'Enciclopedia italiana, dedicata al Diritto, sia per l'Ottocento che per il secolo breve sono state intitolate alla "giustizia penale». ${ }^{\mathbf{1 1}}$ Nelle storie e nelle cronache del rito penale molto hanno contato quelle di chi guidava la «macchina»; ${ }^{12}$ le vicende della giustizia sono state indissolubilmente legate a quelle dell'ordine giudiziario. Se nell'Italia unita quel «potere» nasceva «debole», ${ }^{13}$ proprio grazie al processo penale acquistava visibilità e si guadagnava uno spazio costituzionale, decisivo

6 Carrara (1878) 17. Su Carrara anche per indicazioni cfr. ora Orlandi (2006) 293 ss.; Lacchè (2007a) 662 ss.; De Francesco (2012) 357 ss.; sia consentito di rinviare anche a Colao (2013a) 463 ss.

7 Miletti (2003).

8 Pisapia (1989a) 77 ss. Analogamente Grevi (1997) 255 ss.; Pisani (2009) 139 ss.

9 Manzini (1956) I, 107. Sul "codex unius authoris» cfr. Miletti (2010) 63 ss; Sbriccoli (2009b) 1001 ss.

10 LACChè/MecCARELli (2012a) 7.

11 Per un'analisi della giustizia penale dall'Unità al codice del 1913, epilogo di «retoriche dello scontento e garanzie tradite» cfr. MiLetri (2012) 467 ss.; sulla periodizzazione del secolo breve in età giolittiana, regime fascista, età repubblicana, prospettive aperte nell'ultimo scorcio di fine 900 cfr. Orlandi (2012b) 675 ss.

12 Costa (2012) 38. Sulle dense considerazioni di Pietro Costa su una storia «assai complicata, come pluriverso ed il suo orizzonte metastoriografico», indicazioni bibliografiche anche dei lavori in corso, in Italia e fuori cf. Costa (2012) 23 ss., 37 ss.

13 «La magistratura entrò nella storia d'Italia unita come potere debole»; cfr. Alessi (2001) 165 . 
nella società tra Ottocento e Novecento, ed anche oltre, con la giurisdizione come attuazione della legalità, nel crinale conflittuale tra magistratura e potere politico. ${ }^{14} \mathrm{La}$ storia processuale nazionale è stata poi segnata dal rapporto tra processo penale e opinione pubblica, valore e criticità dalla dimensione politica. Su questo terreno la giuria, sia pure dominata dal presidente delle Assise, anche nel collasso della separazione fatto-diritto, ${ }^{15}$ nel tempo ha perso la centralità ed il senso liberale, peraltro debole e irriducibile al più consolidato modello teorico inglese, ${ }^{\mathbf{1 6}}$ a favore della scelta, avviata dal fascismo, e confermata dall'Italia repubblicana, per il «predominio degli uomini in toga nella gestione del potere giurisdizionale». ${ }^{\mathbf{1 7}}$

A segnare il destino dei modelli radicati fuori dello stivale, tra tutti l'accusatorio "puro», mitizzato in una dimensione fuori del tempo, sono parsi la «scoperta della verità ed il suo "monopolio pubblico», ${ }^{18}$ banco di prova della polarità tra garanzie individuali, cardine teorico dell'ordine liberale, e l'inossidabile cifra pubblica del processo penale anche italiano. Più che nella dimensione soggettiva le "guarentigie» erano declinate dallo stesso Carrara come «tutela giuridica dei cittadini», ${ }^{19}$ assorbite nello ius puniendi della codificazione del fascismo, ${ }^{20}$ spesso disattese anche nell'Italia repubblicana. ${ }^{21} \mathrm{E}$ dunque, nell'impensabilità di idealtipi puri, ${ }^{22}$ la storia del processo penale in Italia è parsa ridimensionare la rappresentazione di un inquisitorio o misto - il codice del 1930 - adatto ai regimi autoritari, e di un accusatorio iscritto nella democrazia, nodo della giustizia politica negli Stati democratici, già colto da Otto Kirchheimer. ${ }^{23}$ Sono insomma i concreti orizzonti della

14 Di recente, anche per indicazioni, cfr. ora Meniconi (2012).

15 Meccarelli (2008) 166 ss.

16 Lacchè (2008) 77 ss.; Cianferotti (2008) 15 ss.

17 Amodio (1979a) 3.

18 Così Garapon (2007) 161. Sul punto, anche per un raffronto con l'esperienza americana, disinteressata al paradigma della verità, cfr. tra gli altri Foucault (1994), particolarmente 28 ss.; Ferrua (1993) 212; Resta (2004) 369 ss.; Taruffo (2009); Incampo/Garofoli (2012).

19 Carrara (1878) 17 ss.

20 Cfr. la efficace sintesi di Neppi Modona (2007) 364 ss.

21 Orlandi (2011) 74 ss.

22 Una riflessione sui «topoi storiografici» su accusatorio e inquisitorio in CAPpellini (2010) 25; Dezza (2011) 101 ss.; Alessi (2011) 349.

23 O. Kirchheimer affermava che l'azzeramento delle garanzie per il nemico politico non era propria dei soli regimi totalitari, ma si ripeteva nella democrazia americana; cfr. Political Justice (1961), e Politik und Verfassung (1964), ora in KirchHeImer (2002) 7. 
storia a insegnare la storia del processo: se a metà anni Settanta Amodio indicava la scelta impervia di un rito senza istruttoria per andar oltre il "garantismo inquisitorio», ${ }^{24}$ nel 2009 Vassalli riconosceva a quella sorta di ossimoro l'attitudine ad aver «retto il processo penale per più di trenta anni, dal 1955 al 1988». ${ }^{25}$

\section{Il processo nell'Italia liberale}

Negli anni Settanta dell'Ottocento Luigi Lucchini si guadagnava un primato tra i processualpenalisti liberali pubblicando una densa monografia, intesa a rivendicare fin entro la istruttoria, dalla cifra inquisitoria, la Pubblicità, oralità e contraddittorio, cardini dell'accusatorio, riconosciuti dallo Statuto. ${ }^{\mathbf{2 6}}$ Con quella scelta sarebbe entrato in crisi il modello misto; lo stesso Carrara metteva in guardia dall'inseguire astratte modellistiche, e raccomandava ai «nostri legislatori» di non nutrire troppa fiducia nel «processo orale», e di non spogliare delle garanzie quello «scritto», dal momento che il dibattimento difficilmente ribaltava i risultati di un «iniquo procedimento». Il giurista toscano asseriva che un modello accusatorio puro avrebbe potuto funzionare solo sotto un «reggimento politico estremamente libero». Era consapevole dei limiti dell'ordine liberale, iscritti nella polarità tra presunzione d'innocenza e tutela della società, e che trovava un terreno d'elezione nel carcere preventivo, in ultima analisi giustificato per la difesa della società dallo stesso Carrara, pur appassionato critico della «immoralità». ${ }^{27}$ Più in generale dalle pagine di Enrico Pessina ${ }^{28}$ all'ampia monografia di Silvestro Graziano, pubblicata alle soglie del codice del $1913,{ }^{29}$ anche alla luce della comparazione l'istruttoria appariva la parte più infelice del codice del 1865, per comprimere i diritti dell'imputato. D'altro canto la soluzione non era

24 Ricorda di aver adottato la felice formula su «Democrazia e diritto» a metà anni Settanta Amodio (2003a) 116.

25 Sul «garantismo inquisitorio che ci ha retto per più di trenta anni, dal 1955 al 1988» cfr. i rilievi mossi ad Amodio da Vassalli (2010) 16.

26 Lucchini (1873). Su Lucchini cfr. Sbriccoli (2009a) 105 ss.; Latini (2012) 362 ss.; Miletti (2013a) 1207 ss.

27 Carrara (2005) 33. Su Carrara di fronte all'accusatorio puro e al carcere preventivo cfr. Lacchè (2007b) 108.

28 Pessina (1904). Su Pessina cfr. Miletti (2013b) 1554 ss.

29 Graziano (1912). 
cercata nel superamento dello schema codicistico anche italiano, mutuato dall'ordonnance d'instruction criminelle, ma nell'ampliare lo spazio della difesa, con una «mezza pubblicità e mezza oralità e guasti ad esse connessi nella struttura bifasica del processo misto». ${ }^{\mathbf{3 0}}$

L'irrompere del positivismo criminologico nella scena culturale innovava il discorso sulla procedura nazionale, cui si affidava al processo un senso diverso da quello della "giustizia dei galantuomini». ${ }^{31}$ Già con L'uomo delinquente di Lombroso ${ }^{32}$ si radicava la lezione positivista tesa a spostare il rito fuori dagli schemi dell'individualismo liberale, che fino ad allora aveva modellato il processo nel segno dell'accertamento giudiziale delle responsabilità, espressa secondo parametri astratti e normativi. Con i Garofalo ed i Ferri si imponeva un diverso orizzonte, la totale sostituzione della prevenzione alla retribuzione, della pericolosità alla responsabilità, col giudizio tappa di un procedimento di valutazione della futura attitudine dell'imputato a nuocere. ${ }^{33}$ L'accento posto sulla difesa sociale costringeva, tra l'altro, a ripensare il ruolo della giuria, dal carrariano "giurì complemento necessario della libertà» ${ }^{34}$ ad arma spuntata contro la criminalità ed artefice di errori giudiziari. $^{35}$

Il Novecento si apriva con le critiche dei processi «indiziari ed infiniti», ${ }^{\mathbf{3 6}}$ dei fallimenti delle istruttorie, dei dibattimenti «ratifica del processo scritto», criticità chiare allo stesso guardasigilli Finocchiaro Aprile, impegnato nella codificazione da tempo in fieri. ${ }^{37}$ L'ordine giudiziario, investito dalla democratizzazione e mediatizzazione, iscritte nell'incipiente società di massa, esprimeva una forte vocazione moralizzatrice e disciplinatrice della giustizia. Per Mortara - tra i protagonisti anche del discorso sul processo penale - lo «Stato democratico» implicava una «relazione tra nazione delegante e i

30 Cfr. Ferrajoli (1989) 636.

31 LACCHÈ (1990).

32 Lombroso (1876). Su Lombroso cfr. ora Marchetti (2009) 1050 ss. e (2012) 69 ss.

33 Garofalo (1892) 199 e (1881); Ferri (1883) 7 ss. Sull'apporto dei maestri della Scuola positiva al dibattito sul processo penale cfr. Miletti (2003) 315 ss.; Stronati (2012a) 97 ss. e (2012b) 371 ss.; Pifferi (2013b) particolarmente 254 ss.

34 Carrara (1883) 356. Sul tema cfr. Da Passano (1989) 257 ss.; Lacchè (2008) 77 ss.

35 Indicazioni in STORTI (1998) 361 ss.

36 Marmo (2003) 113 ss.; Lacchè (2006) 459 ss.

37 STORTI (2008) 138. 
poteri ad essa delegati», ${ }^{38}$ nell'intreccio tra «coscienza del paese e potestà di condannare». ${ }^{39}$

Al termine di un itinerario sofferto, condizionato dalle contrapposizioni di scuola, il livello liberale del codice del 1913 si risolveva nel «ridurre il segreto a più angusti confini», aprendo l'istruttoria al controllo giurisdizionale ed all'intervento del difensore negli esperimenti giudiziali, perizie, perquisizioni domiciliari, ricognizioni. A differenza del pubblico ministero col Procuratore generale «centri motori del movimento giudiziario» ${ }^{\mathbf{4 0}}$ - il patrono era escluso dall'interrogatorio e dalle deposizioni testimoniali, col relegare lo spazio della difesa tecnica a «istruzione compiuta». ${ }^{41}$ Esulava dall'orizzonte codicistico che la prova dovesse formarsi nel contraddittorio; a proposito delle questioni ai giurati poste dal presidente, la Relazione al Progetto asseriva infatti che «è nelle prove, e non nel dibattimento, che il giudizio deve trovare il suo fondamento». ${ }^{42}$

La codificazione non spegneva i contrasti sul senso del processo: Lucchini lamentava la «equivoca supremazia del PM» nell'istruzione sommaria e l'«ermafroditismo del giudice istruttore» ${ }^{43}$; inaugurando l'anno giudiziario 1914 come procuratore generale in Cassazione, Mortara lamentava «la mitezza sistematica delle repressioni». ${ }^{44}$ La mediazione tra ordine e libertà, tra presunzione di non colpevolezza ed esigenze processuali di verità, si traduceva nell'introdurre, per la prima volta in Italia, l'istituto della scarcerazione per decorrenza dei termini, da sei mesi ad un anno per i delitti più gravi, tempi ben più brevi che in futuro. In questo senso nel 1966 Bettiol lodava nel codice Finocchiaro Aprile «una delle più marcate e tipiche espressioni dell'ideologia politica liberale nel campo della legislazione». ${ }^{45}$ In quell'anno Cordero pubblicava Ideologie del processo penale; a confronto col modello inteso ad «escludere dal processo ogni prova ottenuta fuori del controllo

38 Mortara (1992) 188 ss. Per indicazioni su Mortara, anche con riferimento al processo penale cfr. MecCarelli (2008) 178 ss. e (2012) 459 ss.; PiCARdi (2013) 1383 ss.

39 VACCA (1912) 529.

40 Lombardi (1914) 323.

41 Artt. 195-198. Sulla pubblicità istruttoria cfr. Miletti (2007) 901 ss. e (2008) 227 ss.

42 Gismondi (1914) 217.

43 Lucchini (1913) 681 ss. Sull'art. 277 cfr. Storti (2011a) 592. Sul «difetto d'origine» dell'istruzione sommaria, introdotta dal codice del 1913 cfr. Cordero (1997a).

44 Mortara (1914) 1857 ss.

45 Bettiol (1966) 173. 
delle parti» - un «accusatorio», in cui «la caccia vale più della preda e cioè il modo in cui si agisce conta più del risultato» ${ }^{\mathbf{4 6}}$ - il codice del 1913, pur meno «oppressivo» di quello del 1930, «moderatamente liberale», dalle «pallide garanzie», pareva «disinnescare ogni autentica valenza accusatoria». ${ }^{47}$

\section{Il processo al tempo del fascismo}

A differenza che nell'Italia liberale e repubblicana il codice di procedura penale del 1930 era contestuale al codice penale ed all'ordinamento penitenziario, col processo in un ruolo di cerniera, «strumento di attuazione dei comandi del codice penale». ${ }^{48}$ La torsione autoritaria del codice precedente, definito dallo stesso Manzini «opera pregevole, che preparò la codificazione vigente», ${ }^{49}$ era realizzata soprattutto col dare vigore al protagonista del processo, l'organo dell'accusa. Lo spunto accusatorio proposto da Rocco nel discorso del 1925 al Senato - «si restituisca al pm intera la sua qualità di parte» - era inteso a ribaltare l'impostazione del codice Finocchiaro Aprile, che aveva escluso il pm dal novero delle parti, in quanto strumento di una sentenza «giusta», non solo di «condanna». ${ }^{50}$ Nel Progetto definitivo, anche in risposta ai rilievi della dottrina, il guardasigilli specificava di aver spiegato che attribuire al pm il carattere di parte non significava negarne il carattere di «organo pubblico» e bollava la questione come «accademica». Nel codice al pm sarebbe rimasta la facoltà di produrre anche prove a discarico dell'accusato in quanto "parte sui generis, che agisce nell'interesse dello Stato, per un fine di diritto pubblico, senza una situazione di uguaglianza di fronte all'imputato». ${ }^{51}$

Nella giustizia che il totalitarismo italiano disegnava con la codificazione processulpenale si irrobustiva dunque il carattere originario del pm «figlio della politica», col controllo del governo sulle procure, destinato ad aumen-

46 Cordero (1997c) 205-206. Osserva che «chi lesse quella frase anche una sola volta, non può averla dimenticata» NobiLi (2009) 285.

47 Cordero (1997d) 247 e (1986) 94-95. Di recente per due diversi giudizi sul codice del 1913, sotto il profilo politico, cfr. Caprioli (2011) 501 ss.; Natale (2013).

48 Discorso (1931) 18. Su Longhi cfr. Miletti (2013c) 1195 ss.

49 Manzini (1931a) 66.

50 Rocco (1925) 39 ss. Sul dibattito in seno alla dottrina cfr. Miletti (2008) 247 ss.

51 Rocco (1930) 22. 
tare con la riforma Grandi dell'ordine giudiziario, che avrebbe eliminato il controllo giurisdizionale sull'archiviazione ed assegnato al pm il «dominio dell'azione penale». ${ }^{52}$ L'accusato era pensato come indiziato di reità, destinato a trascorrere in carcere tutti i gradi del processo, malgrado esso potesse chiudersi con una sentenza di assoluzione. La prova era raccolta nell'ipertrofica istruttoria: la preliminare, affidata alla polizia, la sommaria al pm, la formale al giudice istruttore, con la difesa tecnica ammessa dopo la publicatio processus. ${ }^{53}$

La «continuità di carattere e discontinuità ideologica della codificazione» - di cui ha scritto Sbriccoli ${ }^{54}$ - ci sembra una chiave di lettura convincente per non cogliere del lavoro di Manzini solo un ovvio, seppur vistoso, arretramento dei diritti individuali. Pare inoltre fuorviante considerare la tenuta delle garanzie procedurali entro un regime che andava sottraendo alla giurisdizione tutta la sfera del dissenso politico, per affidare una sorta di repressione preventiva all'Ovra, al Tribunale speciale, a quello della razza. ${ }^{55}$ Così come rispetto all'età liberale anche grazie alle Circolari cresceva inevitabilmente il controllo dell'esecutivo sul magistrato, nell'aula e fuori, fino al passaggio «dalla separazione dei poteri alla divisione di funzioni». ${ }^{56}$

Sul piano delle ideologie, destinate a farsi mentalità, il regime costruiva un processo «a immagine del fascismo, testimonianza e metro delle condizioni in cui costringeva la società italiana», ${ }^{57}$ offrendo veste giuridica al principio della politicità integrale dell'esperienza individuale e collettiva. Il legame con l'opinione pubblica era ripensato da Rocco nei termini della «sentenza, espressione dei sentimenti che il delitto ha suscitato nell'anima popolare», con la giurisdizione specchio fedele «dei sentimenti della massa del popolo». ${ }^{58}$ L'asse della procedura era spostato dunque dall'individuo alla pretesa punitiva dello Stato; nella densa Premessa al Trattato del Manzini Rocco esaltava la nuova cifra politica del codice, «nuovo» rispetto all'«incon-

52 Discorso (1939) 731.

53 Sul codice Rocco, ricompreso nella storia del processo italiano, prima e dopo il fascismo cfr. Alessi (2001) 197 ss.; Neppi Modona (2007) 364 ss.; Miletti (2010) 57 ss.

54 Sbriccoli (2009d) 528.

55 Neppi Modona (2010) 209 ss.

56 STORTi (2011a) $630 \mathrm{ss.}$

57 SBriccoli (2009e) 991.

58 Rocco (1931a) 601. Sul tema Orlandi (2010) 255 ss. 
trastato dominio delle ideologie liberali», premessa alla debolezza degli ordinamenti processuali $[\ldots]$ la giustizia penale in balia delle parti». ${ }^{59}$ Non a caso la circolare del 1931 sui poteri del pm invitava a «bandire fin dalla prima applicazione del codice ogni malinteso pietismo, il quale vulnererebbe in pieno la finalità della nuova legislazione penale». ${ }^{\mathbf{6 0}}$

La scienza e la giurisprudenza, che certe scelte codicistiche avevano anticipato, si accodavano al regime nell'esaltare i profili politici del processo. ${ }^{61} \mathrm{Si}$ apprezzava la "giuria del nuovo Stato», ove i cinque giurati votavano assieme ai togati; ${ }^{62}$ il disciplinamento della libera parola dell'avvocato, affidato al giudice dell'udienza, nel superamento del defensor rei dell'età liberale; si profilava un passaggio dalle proposte del Carrara e del Lucchini di un «Tribunato della difesa», a quelle, sia pure perdenti, del difensore inquadrato nell'Ordine fascista, «pubblico ufficiale». ${ }^{63}$ La previsione che voleva sanabili tutte le nullità, anche quelle relative all'esercizio del diritto di difesa, era corollario della torsione autoritaria dell'antiformalismo, il «sopravvento» nelle parole di Rocco - della «giustizia sostanziale su quella meramente formale». ${ }^{64}$

Oltre a questi dispositivi processuali, il fascismo avrebbe lasciato alla giustizia repubblicana un ideario intriso dell'ossessiva ricerca della verità materiale, dell'ontologica pericolosità dell'imputato, del rifiuto - comune alla dottrina prima durante e dopo il regime - dell'ideologia della «società semplice spettatrice della lotta drammatica tra pretesa punitiva dello Stato e la difesa dell'accusato». ${ }^{65}$ Oltre all' «inconscio inquisitorio», chiave di lettura per spiegare la «eredità di Codice Rocco», ${ }^{\mathbf{6}}$ il bisogno di «giustizia attiva» ${ }^{\mathbf{6 7}}$ avrebbe complicato la fondazione del processo sui «diritti individuali». ${ }^{68} \mathrm{Il}$

59 Rocco (1931b) IX-X.

60 Sulle attribuzioni degli organi del Pubblico Ministero (1931) 219.

61 Sulle «convergenze reazionarie» cfr. MiLetTi (2010) 84 ss.

62 Амоdio (1979b) 3.

63 Anche per indicazioni cfr. Meniconi (2006).

64 Relazione del Guardasigilli al progetto preliminare (1930) XXXII. L'accento sulla giustizia sostanziale serviva a legittimare la scure sulle nullità; sul punto cfr. Dezza (2010) 167 ss.

65 LONGHi (1925) 209.

66 Garlati (2010a) 1 ss.

67 Per un'attenzione al concreto funzionamento del processo, correlato alle forme di Stato, cfr. Damaska (1991) 46 ss.

68 Cappellini (2011) 52 ss. 
senso del processo penale come difesa della società - nel meno crudo lessico del legislatore repubblicano «tutela della collettività» ${ }^{6}$ - non avrebbe cessato di circolare nella società italiana e nella sua prassi processuale. A proposito «delle tradizioni, che lasciano un residuo vischioso di abitudini mentali», ${ }^{70}$ lo stesso Manzini ragionava sulla lunga durata di un «procedimento penale non frutto delle meditazioni dei filosofi [...] neppure della politica di un determinato Stato. [...] l'istituto giuridico penale processuale è rimasto sempre identico nelle sue linee fondamentali, nella sua funzione, nella sua ragion d'essere». ${ }^{71}$

\section{Un processo per la Costituzione}

Già dal 1944 l'Italia liberata varava quelle che in seguito sarebbero state definite «riforme antifasciste del codice»; i «decreti legislativi» 14 Settembre 1944, 8 dicembre 1944, 5 ottobre 1945 sottoponevano il fermo di polizia e le perquisizioni al controllo giudiziario, con l'osservanza delle disposizioni garantita da provvedimenti disciplinari; concedevano il ricorso in corte d'appello e in Cassazione contro le decisioni in materia di esecuzione delle misure di sicurezza; ristabilivano le nullità d'ordine generale insanabili; abolivano i limiti temporali all'intervento del difensore in dibattimento; limitavano, sia pure «temporaneamente», i casi di arresto, mandati di cattura e custodia preventiva, con aumento di termini per il giudizio direttissimo; subordinavano al controllo del giudice istruttore il potere di archiviazione del pm e del pretore, cancellando lo strumento chiave del controllo politico del fascismo sul processo. ${ }^{72}$

Ai lavori della Costituente si affiancava la ripresa del dibattito scientifico: nel 1946, con la Costituzione in fieri, Pietro Nuvolone tematizzava la densità costituzionale dei modelli in campo nel delicato «momento penale»: in primo luogo rilevava la polarità tra gli «interessi individuali» e l'«oggetto del processo", la res iudicata, dalla cifra inevitabilmente pubblicistica, ma da svincolare dal «dogma statualista». Rifletteva poi sull'«eclettico agnosticismo dei codici Rocco», la nascente «influenza anglosassone», lontana dal «lega-

69 Conso (1980) 470 ss.

70 Cordero (1997e) 166.

71 Manzini (1931b) 5-6. Si sofferma sul denso passaggio Miletti (2010) 107.

72 Sulle «riforme antifasciste» cfr. Chiavario (1995) 94. 
lismo», la «concezione russa», col diritto «strumento di lotta per l'affermazione di un ideale politico, di partito o di classe». Il pragmatismo della cultura d'oltreoceano pareva poter costringere la scienza nazionale ad aprire la gabbia dello statualismo, cui era stato strumentale il sistema misto. Per Nuvolone la tragica esperienza della dittatura insegnava che $\mathrm{i}$ «confini rigidi del diritto positivo» non avevano offerto "al cittadino nessuna garanzia sostanziale contro gli arbitri dello Stato». ${ }^{73}$

Nel Congresso nazionale promosso a Firenze nel 1947 dal Consiglio nazionale forense - presieduto da Calamandrei - Leone elaborava un ampio schema di riforma, che, nel 1990, avrebbe definito «architrave» delle sue successive «posizioni». Il giurista napoletano guardava ai Nicolini e Carrara come protagonisti di un ordine liberale, ancora provvisto di senso; dichiarava infatti di non volersi muovere «ab irato nei confronti del codice Rocco, da posizione di preconcette antitesi, rispettando la tradizione fin dove questa non si trasformi in un ostacolo al passo deciso che lo spirito democratico dovrà muovere nel campo della giustizia penale». Oltre ad una «nuova disciplina dei diritti del difensore», tra le «innovazioni dei principali istituti» era indicata l'adozione dell'istruzione formale per «tutti i processi», ${ }^{74}$ senza che dell'inossidabile modello misto, inaugurato dal code d'instruction criminelle, si cogliessero le "contraddizioni». ${ }^{75}$

Dopo un ampio dibattito, il Congresso fiorentino sintetizzava alcune «improrogabili riforme», «espressione e presidio di libertà», in grado di «agevolmente inserirsi nella struttura del codice vigente». I relatori, lo stesso Leone e l'avvocato Gaetano Pacchi, ponevano al centro i diritti dell'imputato, nel segno del favor libertatis. Tra le «modificazioni da attuare con la massima sollecitudine» risaltavano il diritto del privato denunciante o querelante a veder promossa l'azione penale o la richiesta di archiviazione al giudice istruttore; l'abolizione dell'autorizzazione richiesta per i reati commessi in servizio di polizia; l'obbligo di comunicazione sollecita del promovimento dell'azione penale. La permanenza dell'imputato in stato di libertà fino a sentenza definitiva era indicata come regola interna al processo; si chiedeva al legislatore di fissare termini perentori alla durata della carcera-

73 Nuvolone (1946) 263 ss. Su questo scritto cfr. Lacchè (2010) 291.

74 LeONe (1990a) 11-52. Su Leone e sulla processualpenalistica in questi anni cfr. OrLandi (2011) 61 ss.; Chiodi (2011) 244 ss.; Miletti (2011) 368 ss.

75 Alessi (2003) 13 ss. 
zione preventiva e di riconoscere la facoltà di impugnare i provvedimenti limitativi della libertà personale. Si insisteva sul dover rafforzare il ruolo del difensore nel segno della parità delle armi, col diritto a partecipare ad atti di ricognizione, perizie, ispezioni, «ed in genere a tutti quegli atti istruttori ai quali il pm chiede di partecipare». ${ }^{76}$

Poggiavano su questa piattaforma tecnica i temi posti alla Costituente, "postulati del giusnaturalismo illuminista»: ${ }^{77}$ la «inviolabilità dagli arresti, il diritto di essere giudicato dai giudici naturali, punito secondo una legge preventiva e con pene legali, il diritto ad una libera ed efficace difesa processuale e presunzione di innocenza fino alla condanna». Con le parole di Aldo Moro la Prima Sottocommissione presentava l'«elenco sistematico dei diritti e doveri dei cittadini», col mettere a fuoco un modello di processo come «forma di garanzia della libertà individuale», della «libertà del cittadino», in primis quella "personale». ${ }^{78}$ La Costituzione coniugava dunque reazione alla statolatria del fascismo e funzione attiva e promozionale delle garanzie, volute per l'imputato e per l'ordine giudiziario. La giurisdizionalità in senso lato era la prima garanzia ordinamentale per lo statuto del magistrato - imparziale ed al tempo stesso coinvolto attivamente nel processo - e si connetteva al principio del giudice naturale, dell'inderogabilità del giudizio, dell'infungibilità della giurisdizione penale e obbligatorietà dell'azione penale. Inteso all'attuazione del diritto penale sostanziale, il modello costituzionale era legittimato sia dalla correttezza dei metodi impiegati che dal realizzare nella vita sociale le scelte politiche della Repubblica. Calamandrei avrebbe riposto nella Costituzione il senso di un giudice vocato ad andar oltre l'esegesi della legge per raccordare la giurisprudenza ai valori della Carta, in una svolta per la giustizia in Italia, "contro il passato, e contro il presente». ${ }^{79}$

La tutela dei diritti individuali conviveva inoltre con il paradigma dell'«intervento attivo e promozionale dello Stato», lontano dall'ideologia dell'accusatorio "puro». ${ }^{\mathbf{8 0}}$ Soprattutto per il principio dell'obbligatorietà dell'azione penale - condizione di uguaglianza per i cittadini e indipendenza del

76 Alpa (2008) 151 ss.

77 Costa (1993) 20.

78 La Costituzione della Repubblica italiana (1970) 307 ss e I, 701 ss.

79 Calamandrei (1953) 167.

80 Costa (2011) 156. 
pm da ogni ordine di poteri ${ }^{\mathbf{8 1}}$ - la Carta del 1948 non guardava infatti ad una «teoria sportiva della giustizia», ad un magistrato impassibile spettatore del «processo di parti». ${ }^{82}$ Se delle garanzie dell'adversary system il «breviario giuridico del popolo» ${ }^{83}$ recepiva il principio di non colpevolezza e della separazione delle funzioni di accusa, difesa, giudizio, pure non contemplava l'ideologia performante la legalità americana, disinteressata alla verità, ${ }^{\mathbf{8 4}} \mathrm{ed}$ un suo corollario, il patteggiamento. ${ }^{85} \mathrm{Il}$ nesso giustizia / verità ed una concezione promozionale delle garanzie erano iscritte nella Carta del $1948,{ }^{86}$ in sintonia con i giuristi del tempo, non disposti a rinunziare questo paradigma. Capograssi guardava alla «magia del processo», 87 Satta al suo «mistero», come ad un far rivivere l'accaduto, con giudizi penali cognitivi in fatto e ricognitivi in diritto, sempre alla «ricerca della verità». ${ }^{88}$ Carnelutti, precoce fautore del superamento dello schema istruzione giudizio, architrave del modello misto, rappresentava un «processo penale [che] non serve a comporre una lite, ma a ristabilire il regno della giustizia turbato dal delitto». ${ }^{\mathbf{9}}$

\section{L'Europa e l'Italia. La Novella del 1955}

Nel 1950 la Convenzione europea per la salvaguardia dei diritti dell'uomo e delle libertà fondamentali poneva all'agenda del legislatore italiano il tema di una riforma della codificazione nel segno delle garanzie processuali, chiesta soprattutto dall'articolo 6, che prevedeva, tra l'altro, il diritto dell'individuo ad una causa esaminata equamente, pubblicamente ed entro un termine ragionevole, la presunzione di innocenza, il diritto ad essere informato, quello di difesa ed autodifesa, quello di esaminare i testimoni. Nelle due lingue ufficiali della Convenzione erano questi i lineamenti del Right to a Fair Trial e del Droit à un procès équitable, tradotti negli anni come «diritto ad

81 Neppi Modona (1987) 39 ss.

82 Sul modello costituzionale da non ricondursi al «quadro tipico del modello accusatorio», cfr. Grevi (2003) XIV.

83 Grossi (2012) 74.

84 Anche per indicazioni cfr. Kagan (2001) 61 ss., (2007) 41 ss. e (2009).

85 Cfr. tra gli altri Gambini Musso (1985); Fisher (2003); Marcolini (2005).

86 Costa (2011) 154.

87 Capograssi (1950) 1 ss.

88 SATta (1994).

89 Carnelutti (1964a) 27; analogamente, Carnelutti (1950a) 35. 
un equo processo», fino alla locuzione "giusto processo», accolta dal legislatore costituzionale nella riforma dell'art. 111 della Costituzione. ${ }^{90}$ Il Consiglio dei ministri d'Europa, che segnalava il deficit di garanzie nel processo italiano, imponeva alla dottrina più sensibile all'impatto della normativa internazionale sulla procedura il tema della «traduzione diretta» delle disposizioni della Convenzione in «specifiche disposizioni di codice». ${ }^{91}$

La Convenzione sarebbe stata resa esecutiva in Italia poco dopo la Novella del 1955, preparata da un grande dibattito legislativo e dottrinale, in cui le garanzie iscritte in una prospettiva comparata erano comunque immerse nella tradizione nazionale. In un denso saggio del 1950 Bettiol legava i termini «accusatorio» e "processo penale democratico» per descrivere un modello per il quale «ogni attività processuale è diretta alla salvaguardia dei diritti fondamentali dell'individuo». Non prospettava peraltro la fuoriuscita da un sistema di cui l'«atmosfera politica antiliberale» non pareva aver «deformato lo «spirito tradizionale», ma «intaccato alcuni singoli istituti in modo da non tutelare rigorosamente la libertà individuale». Affermava che la «riforma» doveva intervenire su quei «singoli istituti», col «criterio di libertà penale come bussola». ${ }^{92}$ Anche una densa Prolusione di Leone guardava al «tradurre in norme legislative le garanzie costituzionali di libertà e democrazia», in vista di un «futuro processo», che era ancora quello «liberale». Non a caso il giurista e politico napoletano trovava nella lezione del Carrara, vista in continuità con la Costituzione, la rappresentazione di due idealtipi, inquisitorio e accusatorio, quest'ultimo improponibile per la «funzione altissima ed insostituibile del pm nella società moderna». Leone coglieva in questo nodo l'ostacolo ad un processo di parti; ma asseriva che qualche aspetto di quel modello poteva contribuire ad un «orientamento democratico del processo penale», in continuità col «rapporto giuridico di tradizione liberale», vocato a «rispettare al massimo la libertà individuale». La accettazione di profili accusatori, volti ad una maggior «efficienza delle parti», ad

90 Il testo in Conso/Riccio (1998) 451 ss. Cfr. inoltre Malinverni (1972) 62. Sulle origini della locuzione dell'art. 6 della Convenzione cfr. Alvazzi Del Frate (2011) 149 ss.

91 Chiavario (1991c) 527 ss., che ricorda un suo saggio risalente al 1964. In precedenza cfr., tra gli altri, ANDrioli (1965) 17 ss.; Amodio (1967) 841 ss.; ricorda i suoi risalenti interventi Conso (1969a) 108 ss. Più di recente cfr. Amodio (2003a) 88 ss.; Chiavario (1998) 52; Chiodi (2011) 249.

92 Bettiol (1950) 126 ss. 
esempio col ripristino delle nullità assolute, lasciava intatto lo schema bifasico e la «funzione pubblica del processo penale, che non può essere nè sfaldata nè diminuita». ${ }^{93}$

In un importante Convegno del 1953 la processualpenalistica precisava il senso dei modelli per il processo nazionale. Carnelutti metteva a fuoco il nucleo della sua «bozza» del 1962: l'opzione dell'inchiesta preliminare, di derivazione civilistica, da sostituire all'istruzione. ${ }^{94}$ Vassalli argomentava che la proposta Carnelutti andava a colpire il principio del libero convincimento, ed asseriva che il «magistrato (e con lui accusa e difesa) devono poter vedere e vagliare le prime fonti della notizia di reato». L'introduzione di «limiti legali di durata di carcerazione preventiva in ogni fase» pareva l'unica soluzione praticabile «in Italia», una scelta certo più modesta dell'auspicato «habeas corpus $[\ldots]$ immediato controllo sulla legittimità della cattura da parte di un organo diverso [...] troppo lontano dal nostro sistema processuale vigente». ${ }^{95}$ Era la Relazione di Pisapia sull'intervento della difesa nell'istruzione a centrare il problema: nell'impossibilità di una «radicale riforma di struttura», bisognava contentarsi di «iniezioni di libertà ad un codice che, soprattutto per quanto concerne la fase istruttoria è decisamente inqusitorio, e come tale, antidemocratico». ${ }^{96}$ Con le parole di Leone, Pisapia affermava che rafforzare i diritti della difesa e moderare quelli del pm avrebbe adeguato al dettato della Costituzione la «pretesa punitiva dello Stato». ${ }^{97}$ Entro un modello «misto», che pareva comune a tutti i paesi, Pisapia auspicava una «tendenza» verso l'«accusatorio, tipico dei regimi democratici», declinato nel segno del favor libertatis, ad ispirare nuove norme in tema di mandato di cattura, custodia preventiva, scarcerazione automatica. ${ }^{98}$

Carnelutti interveniva per evocare una "grossa eresia», il superamento dello schema bifasico; poneva pertanto al legislatore il tema di una scelta di fondo: «un processo di parti o un processo senza parti», o l'«esperimento» dell'accusatorio, (pericoloso perchè siamo troppo intelligenti in Italia»), o «'inquisitorio».99 La Relazione del sostituto procuratore De Ruggiero pro-

93 LeONe (1964a) 43-75.

94 LEONE (1964a) 51.

95 LeONe (1964a) 59 e 113.

96 LEONE (1964a) 72.

97 LEONE (1964a) 79.

98 LeONe (1964a) 140 ss.

99 LeONe (1964a) 83. 
poneva di «dare al pm un carattere esclusivamente di parte e al giudice un carattere esclusivamente di giudice»; Carnelutti, che pure apprezzava l'accentuazione del «carattere accusatorio della istruzione sulla falsariga del procedimento anglosassone, per cui questa viene a guadagnare in rapidità e semplicità», coglieva che l'eventuale scelta per una «istruzione a tipo accusatorio» esigeva la «collaborazione di ambo le parti all'istruzione [...] implica in ciascuna di esse una devozione al pubblico interesse, che non si può ritenere ancora matura nella presente fase della cultura giuridica italiana». ${ }^{\mathbf{1 0 0}}$

Nel dibattito teorico e nei progetti del legislatore si imponeva il disegno di Leone, forte di una indiscutibile qualità tecnica; la tensione per una «impostazione più liberale del processo» si risolveva in una via compromissoria, mista, di una «nuova più idonea sintesi tra l'indefettibile carattere pubblicistico del processo penale ed il riconoscimento degli interessi e dei diritti dei privati, intendendo per privati non solo l'imputato, bensì l'offeso e il danneggiato dal reato». A sbarrare la strada all'accusatorio, che pareva incompatibile col profilo "pubblicistico» del processo, era dunque determinante la cultura della "pretesa punitiva dello Stato», di cui si poteva magari «moderare la forza», col rafforzare $\mathrm{i}$ «diritti della difesa». ${ }^{\mathbf{1 0 1}}$ Era questo il cuore vitale della Novella del 1955, vocata all'attuazione della Costituzione nel segno delle garanzie pensabili nel modello misto, e che il guardasigilli, De Pietro, avvocato, indicava come una soluzione in grado di rispondere alla diagnosi già corrente sul processo "grande malato»; col presentare la sua ricetta asseriva che «dalla malattia alla guarigione c'è la convalescenza». ${ }^{\mathbf{1 0 2}}$

La riforma era in sintonia con le proposte di Leone soprattutto nell'ammissione del diritto di difesa fin nella «istruzione attuale, tutta ispirata al sistema inquisitorio", salvo che per i primi interrogatori dell'imputato; la «custodia preventiva, che risponde a talune innegabili esigenze», era ristretta rispetto al codice; fermo di polizia, arresti e altri provvedimenti restrittivi erano sottoposti al vaglio del giudice. I discorsi inaugurali dei Procuratori generali erano quasi senza eccezione critici della Novella, accusata di favor $r e i$, di «disarmare la società». Si argomentava che la «libertà» non doveva ricevere tutela «oltre certi limiti», pena «frapporre ostacoli all'opera della polizia e a quella dell'inquirente con limitazioni», dal momento che «con-

100 LeONe (1964a) 328-329.

101 LeONE (1964c) 117.

102 D’Aniello (1955) 8, 10 e 190.

256 Floriana Colao 
tenere presunti eccessi può impedire che luce sia fatta». Nel migliore dei casi la legge era contestata per «appesantire il corso della giustizia»; anche, chi riconosceva al legislatore il merito di essersi ispirato ai "precetti della Carta costituzionale», sosteneva che la riforma «nell'attuarla ha obbedito troppo ad esigenze teoriche». ${ }^{\mathbf{1 0 3}}$

La processualpenalistica guardava invece con favore alla legge del 1955: con la «allargata partecipazione della difesa agli atti più importanti della istruzione, questa scolora un poco i suoi caratteri prima decisamente inquisitori; e codesto non è un vantaggio da poco. ${ }^{104}$ Leone definiva la riforma una «attuazione della Costituzione [...] entro un inevitabile sistema misto», ancorato però all'ideologia del «codice degli innocenti». ${ }^{105}$ Ribadiva inoltre la dimensione pubblicistica delle garanzie, «tutte quelle regole che assicurano la più ampia libertà di difesa non vanno intese come privilegio di soggetti privati, bensì come l'attuazione di un interesse della società». ${ }^{\mathbf{1 0 6}}$

Era ben diverso il giudizio dell'avvocato Adolfo Battaglia; Processo alla giustizia denunziava il «codice del 1931» come «il più fascista», tappa di un «estremo grado di corrompimento del sistema misto», accusato, con una serrata rassegna di esempi concreti, di «violare i diritti di libertà» ed «alimentare le speranze di impunità dei cittadini peggiori». ${ }^{\mathbf{1 0 7}} \mathrm{Nel}$ nesso processocostituzione, Battaglia criticava, anche sulla stampa, molti profili della Novella del 1955, che pareva «codificare la lungaggine», col compromettere gli stessi diritti dell'imputato, che si dovevano «meglio garantire». Ai «paesi che si vantano di aver costruito, con il processo Dreyfus e con il processo Cuocolo, i più colossali edifici processuali», l'avvocato scriveva di preferire il «paese che ignora addirittura il fascicolo degli atti e nel quale, dinanzi al presidente non figura mai nessuna carta». Alla logica del «processo perfetto» anteponeva quella del «processo immediato»; a quella dell'«inquisitore giudice e presidente inquisitore», che ritengono di poter raggiungere la «verità per proprio conto", preferiva il "gioco delle parti», cardine del modello epistemico «anglosassone», colto nella valenza «liberale e autonomista» di

103 Sui Procuratori generali davanti alla legge del 1955 cfr. Leone (1990a) 96.

104 Bellavista (1956) 45.

105 LeONE (1964a) 5.

106 LeONe (1955) VII.

107 Battaglia (1954) 5 e 226. 
una società, e dunque di una giustizia, ancorata al pensiero dei Tocqueville e Stuart Mill, «individualista e libertaria». ${ }^{\mathbf{1 0 8}}$

\section{6. «Diatribe sul processo accusatorio»}

Nel 1961 Carnelutti non proponeva il superamento dello schema bifasico per adeguare il processo alla Costituzione, alla Convenzione europea dei diritti dell'uomo, a modelli anglosassoni, non troppo apprezzati dal giurista friulano; la proposta di riforma voleva esser la prova della raggiunta maturità scientifica della processualpenalistica nazionale, Cenerentola, condizione per una radicale cura legislativa del «grande malato». Essa risiedeva in una svolta: due fasi del rito erano nettamente separate; per la prima, libera da forme, non si escludeva la segretezza e l'assenza di difesa. A garanzia dell'indagato la «bozza» prevedeva che le risultanze dell'inchiesta preliminare di parte non potessero essere esibite nel dibattimento, per «garantire il contraddittorio nella formazione delle prove». ${ }^{109}$

Nel Convegno promosso nella primavera e nell'autunno 1964 tra Lecce e Bellagio dal Centro nazionale di prevenzione e difesa sociale la Relazione di Cordero tematizzava una dimensione «ideologica» dell'alternativa inquisitorio e accusatorio; e di quest'ultimo profilava le «linee», col superamento del «processo a due tempi». ${ }^{\mathbf{1 1 0}}$ Era il tema cruciale della prova ad imporre questo radicale mutamento di paradigma: «nessun giudizio storico è tale che sia assolutamente impossibile predicare il contrario [...] il concetto di verità processuale si può ottenere soltanto a prezzo di una determinazione quantitativa delle probabilità contrarie». ${ }^{\mathbf{1 1 1}}$ In sintonia con l'epistemologia degli anni Sessanta a Cordero premeva un processo ove la «interrogazione incrociata» facesse cadere «il diaframma tra parte e prova»; l'«inchiesta preprocessuale» produceva un "precedente storico», e l'unica prova ammissibile si formava nel contraddittorio, con la fine del rito «sommerso dalle scritture», ${ }^{112}$ del giudice dalla «vorace potenza superlogica». ${ }^{113}$ A differenza del

108 Battaglia (1988) 189 ss.

109 Carnelutti (1962a) 15-16 e (1962b) 1 ss. Sulla bozza Carnelutti anche per indicazioni cfr. Nobili (1998) 70; Cappellini (2011) 3; Storti (2011b) 179.

110 CoRdero (1997e) 159 ss.

111 CORDERo (1963) 45.

112 Cordero (1997e) 78.

113 Intervento, in Criteri direttivi (1965) 213. 
«paradigma anglosassone», il magistrato non era peraltro inteso come «muto spettatore», ma come protagonista nel valutare, a richiesta dell'imputato, il «fondamento dell'accusa». ${ }^{\mathbf{1 1 4}}$

Nel dibattito che si apriva Nuvolone accusava gli innovatori di avere «'accusatorio sul labbro e l'inquisitorio nel cuore»; opponeva poi un argomento cruciale al principio dell'esclusione del valore probatorio di quel che acquisito nell'inchiesta preliminare, di "prove che non avranno valore di prove». Affermava che le «particolari condizioni del nostro paese», specialmente la «piaga dell'omertà» davanti al forme di «criminalità organizzata», rendevano «indispensabili gli accertamenti predibattimentali». Nuvolone mostrava inoltre la vanitas di un idealtipo anglosassone di «processo costituzionalmente e psicologicamente diverso dal nostro", con l'inchiesta di parte del pm da cui si potevano difendere solo «imputati ricchi», assistiti da «costose agenzie investigative», col risultato di «legalizzare le sperequazioni sociali nell'amministrazione della giustizia». In quello che pareva l'orizzonte della Costituzione si doveva "perfezionare l'istruzione formale» nel segno della giurisdizionalizzazione e del diritto di difesa. ${ }^{\mathbf{1 1 5}}$ Per Leone un processo accusatorio «vero», con l'indagato davanti al giudice senza soluzione di continuità con la notitia criminis, senza possibilità di proscioglimento o archiviazione, non pareva «possibile oggi nel nostro paese». ${ }^{116}$

La magistratura esprimeva un netto dissenso circa l'inutilizzabilità nel giudizio delle prove raccolte. ${ }^{117} \mathrm{Al}$ favor libertatis, che pareva il cuore vitale del «tipo accusatorio», i magistrati opponevano la «difesa sociale», la «funzione esemplare», i «limiti della verità», il «colpire il delitto» come valori cardine, seppur non tematizzati come alternativi al «proteggere l'innocente». A taluni giudici le Relazioni dei «professori» parevano stare alla "pratica» come il «telescopio al microscopio»; si marcava la distanza tra «evoluzione» e «rivoluzione». ${ }^{118}$ Con una precoce attenzione al nesso Law and Film, ${ }^{119}$ si irrideva all'«invincibile Perry Mason"; all'obiezione di un sostituto procuratore - «il processo alla Perry Mason non ci convince, lo conosciamo bene

114 Cordero (1997c) 205-206.

115 Nuvolone (1965) 82-88. Cfr. anche Nuvolone (1964)178 ss.

116 Criteri direttivi (1965) 225.

117 Altavista/ Tartaglione (1965) 103 ss.

118 Criteri direttivi (1965) 211 e 219.

119 Su Law and Literature e su Law and film cfr. Costa (2012) 36. 
attraverso la televisione» - Cordero interrompeva, «mi scusi, non ho la televisione in casa». ${ }^{\mathbf{1 2 0}} \mathrm{Al}$ «ritoccare qualcosa, per non mutare nulla», il processualpenalista opponeva che «quando è in gioco il rispetto della persona non ci si può accontentare di una mezza civiltà». Ripeteva il tema del «contraddittorio nella formazione di tutte le prove», e proponeva l'atto delle tre persone, il bartoliano «mito del triangolo», al posto del «mito del giudice infallibile», della «verità, che porta alla tortura». ${ }^{\mathbf{1 2 1}}$

Questi due diversi punti di vista avrebbero segnato il dibattito dottrinale e legislativo, anche nel momento in cui, dalla metà degli anni Settanta, in Italia si imponeva la realtà di un "processo penale contro» ${ }^{\mathbf{1 2 2}}$ - il terrorismo, la mafia, la camorra, i contro poteri occulti, come la P2, e poi la corruzione politica - un modello impensabile entro l'ordine liberale, ma che poggiava sul consenso della società, alle prese con la "perenne emergenza». ${ }^{\mathbf{1 2 3}}$ Essa poneva una ipoteca sul varo di quello che il Parlamento da anni andava discutendo come «nuovo modello accusatorio»; ${ }^{124}$ i maxiprocessi - dal caso Tortora alla Norimberga della mafia, celebrata nel 1985 a Palermo, ${ }^{125}$ che avrebbe poi visto Andreotti imputato del secolo nella «vera storia d'Italia» ${ }^{\mathbf{1 2 6}}$ - davano il senso della procedura come affermazione di legalità davanti ad ogni ordine di poteri, ed imponevano la rinunzia ad un idealtipo "puro».

In vista della legge delega per il codice Leone contestava la discriminazione tra prove ammesse e non ammesse in dibattimento, in quanto contraria al principio del libero convincimento del giudice e poco compatibile con una realtà italiana di «testimoni con la pistola alla nuca». ${ }^{\mathbf{1 2 7}}$ Non a caso Amodio, attento lettore della «prassi» di accusa e inquisizione, ${ }^{\mathbf{1 2 8}}$ insisteva nel tematizzare i caratteri di un «accusatorio all'europea», per respingere la «sbrigativa e fuorviante immagine continuamente evocata di un processo

120 Criteri direttivi (1965) 263.

121 Criteri direttivi (1965) 357.

122 Cfr. tra gli altri Baratta (1993) 120 ss.; Giostra (1995) 155 ss.

123 Moccia (2000).

124 Amodio (1984) 101 ss.; Chiavario (1984) 427 ss.; per un «modello realistico» cfr. Fortuna (1984) 2314 ss.

125 Indicazioni in Ramat (1985) 259; più di recente Alfonso (2011) 3 ss.

126 La vera storia d'Italia (1995).

127 LeONE (1990b) 149.

128 Амодіо (1988a) 20 ss.

260 Floriana Colao 
tutto calcato sull'esperienza americana». ${ }^{129}$ Cresceva la distanza tra chi vedeva nei «maxiprocessi un vizio capitale: sono inoperabili in termini garantistici, vale a dire non possono giungere a buon esito senza sacrificio per $\mathrm{i}$ diritti di difesa e libertà dell'imputato", e chi, come il guardasigilli Rognoni, riteneva che di fronte ai reati associativi la giurisdizione dovesse innanzitutto rispondere alla "grande preoccupazione della gente». ${ }^{130} \mathrm{Si}$ affidava al codice in fieri anche il compito di «combattere sul terreno giudiziario [...] i contropoteri criminali», ${ }^{\mathbf{1 3 1}}$ col rimandare il «nuovo modello processuale accusatorio nella sua integralità ad un momento migliore, quando la società sarà migliore». ${ }^{132}$ Nella "situazione italiana», un paese senza regole, pareva improbabile una «verità processuale che venga fuori da un gioco dove le regole sono rispettate». ${ }^{\mathbf{1 3 3}}$

All'accusa di Raffaele Bertoni - presidente dell'Associazione nazionale magistrati - di un codice voluto dalla politica per «comprimere in qualche misura la funzione di controllo della legalità che è propria della giurisdizione», ${ }^{134}$ Pisapia opponeva di aver tenuto conto dei «problemi di bilanciamento delle ideologie»; stemperava la «accusatorietà», che il preambolo della legge delega imponeva sì di attuare, ma in coerenza con $\mathrm{i}$ «principi e criteri che seguono»: obbligatorietà dell'azione penale, rifiuto della scissione tra giudice e giuria, spazio alla parte civile, scelte ben lontane dal «mondo anglosassone». ${ }^{135}$ Il legislatore percepiva una «coscienza sociale» avversa alla «teoria sportiva»; spiegava che la giustizia negoziale iscritta nei riti alternativi non comportava «la passività» del giudice, iscritta nell'«accusatorio puro». ${ }^{136}$

129 Амоdio (2003b) 230. Una comparazione tra il processo in America e il progetto italiano in Amodio/Bassiouni (1988).

130 Ferrua (1987) 1662 ss.

131 Neppi Modona (1984) 423 ss.

132 Un nuovo processo penale per una società migliore (1985) 192.

133 De Leo (1984) 148 ss.

134 Bertoni (2009) 154.

135 La testimonianza di un protagonista (2011) 425. Di recente sulla ricezione dei concetti e delle espressioni anglosassoni nel nuovo codice, anche per indicazioni, cfr. RugGierI (2012) 144 ss.

136 Pisapia (1989b); Conso (1989); Pisani (2002a). 


\section{Il primo codice della «Repubblica penale»}

Il codice di procedura penale, entrato in vigore nel $1989,{ }^{137}$ era presentato dal guardasigilli Vassalli in una densa premessa alla traduzione in inglese, che tematizzava un "misto accusatorio», laddove il pm, magistrato, non aveva potere di archiviazione, indagava in funzione di fatti anche favorevoli all'imputato, poteva indicare alle parti temi nuovi, «utili alla ricerca della verità». ${ }^{138}$ Chiavario, tra protagonisti della codificazione, assicurava che il modello accusatorio nazionale non aveva voluto realizzare una «teoria sportiva della giustizia, che premia i giochi di abilità delle parti, per una verità formale». ${ }^{139}$ Amodio argomentava che «la svolta» investiva la «tecnica» del processo, e non la «tutela giurisdizionale», che le parti non disponevano dell'«oggetto del processo», su cui era chiamato a deliberare un giudice non vincolato dalle parti. ${ }^{\mathbf{1 4 0}}$ Anche Cordero coglieva che nel codice «l'idea accusatoria risulta elaborata in una sintassi ignota alle pratiche anglosassoni», e che «l'assetto attuale conserva qualcosa del vecchio». La scelta pareva opportuna «nel quadro organico italiano (codice genetico, culture e subculture, apparati, corporazioni, poteri palesi e occulti)», al punto di considerare «probabilmente nefasta una riforma spinta all'estremo purismo accusatorio». $\mathrm{La}$ «fedeltà dogmatica a tale modello, sotto qualche aspetto povero», pareva impossibile, dal momento che il nuovo «testo» non poteva non scontare il peso dell'eredità della storia della giustizia nazionale, «l'inquisizione forma perversa d'una ricca cultura; amputare tutto sarebbe terapia vandalica». Se, uscendo dal «riformismo sterile», il legislatore aveva varato un «codice nuovo nel repertorio italiano, anzi europeo», di «altissimo significato», a Cordero non sfuggiva che la magistratura avrebbe ostacolato la scelta di fondo del codice, i «procedimenti speciali», il «negozio giuridico-penale», per cui, «correlativamente regredisce l'oracolo giusdicente». ${ }^{\mathbf{1 4 1}}$

137 Sul codice del 1988, che condivideva la sorte di quelli «liberali» del 1889 e del 1913, preparati da lunga gestazione, voluti soprattutto da avanguardie della processualpenalistica «civile», con una "fuga in avanti»; da qui i «ritorni all'indietro», imposti da «settori importanti della giurisprudenza», anche perché il legislatore non era stato capace di fissare «equilibri normativi appena convincenti, in grado di resistere alla prova dell'ultima emergenza» cfr. Lacchè (2011) 96; sul codice Vassalli, che aveva congegnato «sistemi forse più avanzati della nazione e dei suoi giudici» cfr. Alessi (2011) 349.

138 VASSALli (1997) 805.

139 Chiavario (1991a) 9.

140 Амоdio (1988b) XXII ss.

141 Cordero (1997d) 77. 
Alla luce della comparazione per Neppi Modona risaltava la «contraddizione» del codice con la struttura «gerarchico burocratica» della magistratura italiana, inadatta alla "riforma in senso accusatorio», dal momento che il pm, e le forze dell'ordine, oltre che come parti erano concepiti come istituzioni pubbliche, in grado di portare un contributo all'accertamento della verità processuale. L'effetto deflattivo pareva poi vanificato dal limitare la riforma al primo grado; il codice scontava un eccesso di impugnazioni, in alcuni casi sproporzionato alla posta in gioco, nella tensione per un ripetuto esame di merito. In un processo, come quello nazionale, fondato sui controlli giurisdizionali, le logiche dell'accusatorio erano destinate a scontrarsi con la cultura, profonda e radicata, che quella emessa dai giudici di vertice fosse da ritenersi la «sentenza giusta». ${ }^{\mathbf{1 4 2}}$ Anche grazie alla prescrizione, a dieci anni dall'entrata in vigore del codice sarebbe parso evidente il «fallimento della funzione deflattiva», mentre in America il plea bargaing concludeva il $90 \%$ dei processi. ${ }^{143}$ Già nel Settembre 1993 un titolo de Il Sole 24 ore faceva dire a Pisapia «Perry Mason non abitò mai qui»; ${ }^{\mathbf{1 4 4}}$ una più recente analisi comparata ha definito la giustizia italiana «a cross-road between the adversarial and inquisitorial systems». ${ }^{\mathbf{1 4 5}}$

Anche Damaska coglieva che le innovazioni più salienti del codice riguardavano l'ordine giudiziario; affermava che «in accordo con la sua recente apostasia della tradizione europea il codice di procedura penale italiano richiede ora che il fascicolo del giudice del dibattimento contenga solo un minimo di informazioni sui risultati delle indagini preliminari»; da qui i problemi applicativi, per altre diverse «abitudini profondamente radicate». ${ }^{146}$ Il codice aboliva infatti l'ufficio del giudice istruttore, protagonista della giustizia italiana; le indagini - fino a 24 mesi per i casi di criminalità organizzata - erano affidate al pm, parte processuale, privo di poteri di coercizione, ed al tempo stesso organo di giustizia; oltre a «prendere notizia dei reati di propria iniziativa e ricevere notizie di reato", era tenuto a ricercare anche fatti e circostanze a favore dell'indagato, come magistrato della Procura dalla «marcata autonomia». ${ }^{147}$ Non a torto si avanzavano dunque

142 Neppi Modona (1991) 263 ss.; analogamente Orlandi (2012a) 83-84.

143 Neppi Modona (2000) 450 ss.

144 Pisapia (1993) 23.

145 LANGer (2004) 1 ss.

146 Damaska (2003) 107.

147 ZAGREBELSKY (1998) 737. 
critiche garantiste del codice: $\mathrm{i}$ «poteri e funzioni inusitate di politica criminale», assegnati al pm, non parevano bilanciati da quelli del giudice delle indagini preliminari, lontano dal modello della «scuola di Irnerio», «in medio cognoscens», dal momento che il gip non poteva «conoscere», e dunque «non garantire né controllare», col passaggio dal «garantismo inquisitorio» all'«accusatorio non garantito». ${ }^{\mathbf{1 4 8}} \mathrm{La}$ «funzione accusatoria del pm e, con essa, l'ipoteca della fase preliminare sugli esiti del giudizio", disegnata dal codice, non a torto pareva offrire una grande «agevolazione» nei «provvedimenti di Tangentopoli», ${ }^{149}$ epopea non solo giudiziaria sulla corruzione, «unica nel contesto delle democrazie liberali». ${ }^{\mathbf{5 0}}$

«Tutto in Italia sta diventando giustizia penale [...] tutta la magistratura cammina con una gamba sola, quella requirente». ${ }^{151} \mathrm{Nel} 1994$ un magistrato da anni protagonista della scena giudiziaria, Adolfo Beria D’Argentine, fotografava ai Lincei lo stato delle cose al tempo di vicende giudiziarie che parevano dare senso ad una «Repubblica penale». ${ }^{152}$ A proposito del «sistema processuale riformato» il guardasigilli Conso lamentava l'«anomalia di un accusatorio", ove il dibattimento distava anni dalla chiusura delle indagini, ove le parti non ricorrevano ai riti alternativi, contando sulla prescrizione, e la custodia cautelare funzionava da unica pena possibile. ${ }^{153}$ In un importante Convegno, promosso dalla Camera dei Deputati, la realtà processuale repubblicana si rivelava terreno di conflitto di culture, tra chi guardava al processo come messa in scena liberale di una lotta tra uguali, accusa e difesa, e chi lo voleva strumento di applicazione della legge penale e sede di controllo generale di legalità di fronte ad ogni ordine di poteri. ${ }^{154}$ Soprattutto la magistratura offriva questo senso al processo, fin dal tempo in cui «combatteva sul terreno giudiziario» terrorismo, camorra, mafia, poteri occulti, cor-

148 Perchiunno (1996).

149 Orlandi (2011) 75; analogamente Pisani (1995) 167.

150 Anche per indicazioni da ultimi cfr. Galli (2009) 233 ss.; Manes (2009) 243 ss.; Guarnieri (2011) 119 ss.

151 Beria D’Argentine (1994) 441.

152 Garapon/Salas (1998).

153 Conso (1993) 408.

154 Raccoglie gli interventi di protagonisti quali il presidente Scalfaro, gli allora presidenti dell'Associazione nazionale magistrati e Unione Camere penali, oltre a studiosi e giudici Giurisdizione e cultura della legalità (1993); Cotrıno/SARzotTi (1995). 
ruzione, "contropoteri criminali», ${ }^{\mathbf{1 5 5}}$ in nome dell'interesse generale alla «verità». Se quel denso termine pareva assente nel codice, era recuperato dalla Corte costituzionale come cuore vitale dell'accertamento giudiziale: la sentenza 255/1992 scolpiva: «fine primario e ineludibile del processo penale non può che rimanere quello della ricerca della verità». ${ }^{156}$

La n. 111/93, relatore Natoli, era esplicita nel rifiuto di un modello come «tecnica di risoluzione dei conflitti nel cui ambito al giudice sarebbe riservato essenzialmente un ruolo di garante dell'osservanza delle regole di una contesa tra parti contrapposte, ed il giudizio avrebbe la funzione non di accertare i fatti reali onde pervenire ad una decisione il più possibile corrispondente al risultato voluto dal diritto sostanziale, ma di attingere - nel presupposto di un'accentuata autonomia finalistica del processo - quella sola "verità" processuale che sia possibile conseguire attraverso la logica dialettica del contraddittorio e nel rispetto di rigorose regole metodologiche e processuali coerenti al modello». La Corte sottolineava che «a prescindere da astratte modellistiche», in un «ordinamento improntato al principio di legalità (art.25, secondo comma, Cost.) [...] fine primario ed ineludibile del processo penale non può che rimanere quello della ricerca della verità (sentenza n. 255 del 1992)», dato che «è, per la verità, incontroverso che sarebbe contrario ai principi costituzionali di legalità e di obbligatorietà dell'azione concepire come disponibile la tutela giurisdizionale assicurata dal processo penale $[\ldots]$ ne sarebbe tradita la funzione conoscitiva del processo, che discende dal principio di legalità e da quel suo particolare aspetto costituito dal principio di obbligatorietà dell'azione penale». ${ }^{157}$

Nell'ultimo decennio del Novecento ed oltre il processo pareva smarrirsi in un «dedalo delle riforme», ${ }^{\mathbf{1 5 8}}$ col porre alla dottrina il compito di un «recupero del sistema», ${ }^{\mathbf{1 5 9}}$ arduo nel momento in cui il cardine dello Stato nazionale di matrice ottocentesca, la codificazione, nelle parole di Sbriccoli pareva «giunta da tempo al suo punto di dinosauro», ${ }^{160}$ fino a rendere pensabile, per taluni, un «diritto senza codice». ${ }^{161}$ Culmine di conflitti isti-

155 Neppi Modona (1984) 423 ss.

156 Giarda (1993) 61.

157 Su questa «summa del processo penale costituzionale» cfr. Giarda (1993) 61.

158 PisAni (1999) 545 ss.

159 Amodio (2003c) 27 ss.

160 Sbriccoli (2009d) 670.

161 Sotis (2007). 
tuzionali e politici tra Parlamento e Consulta, anche la «costituzionalizzazione del giusto processo» rilevava criticità da «italian style»; ${ }^{162}$ i principi che aumentavano la ispirazione accusatoria del sistema si sommavano ai correttivi all'inquisitorio voluti dalla Costituzione, con aggravare le condizioni dell'eterno grande malato, il «processo all'italiana». ${ }^{\mathbf{1 6 3}}$

\section{Bibliografia}

Alessi, Giorgia (2001), Il processo penale. Profilo storico, Roma, Bari: Laterza Alessi, Giorgia (2003), Le contraddizioni del processo misto, in: Marmo, Marcella, Luigi Musella (a cura di), La costruzione della verità giudiziaria, Napoli: Clio press, $13-51$

Alessi, Grorgia (2011), Populismo, legalità, riforma della giustizia, in: Negri/ PIfferi (2011), Diritti individuali e processo penale nell'Italia repubblicana, Milano: Giuffrè, 347-362

Alfonso, Roberto (2011), Introduzione. Il fenomeno del pentitismo e del maxiprocesso, in: Tinebra, Giovanni et al., Fenomenologia del maxiprocesso: venti anni di esperienze, Milano: Giuffrè, $1-13$

Alpa, Guido et al. (2008) (a cura di), Atti del primo Congresso nazionale giuridico forense del secondo dopoguerra (settembre-novembre 1947), Bologna: Il Mulino

Altavista, Giuseppe, Tartaglione, Girolamo (1965), Considerazioni generali sulla riforma del processo penale, in: Criteri direttivi per una riforma del processo penale, Milano: Giuffrè, 103-118

Alvazzi Del Frate, Paolo (2011), Giustizia e garanzie giurisdizionali. Appunti di storia degli ordinamenti giudiziari, Torino: Giappichelli

Amodio, EnNio (1967), La tutela della libertà personale dell'imputato nella Convenzione dei diritti dell'uomo, in: Rivista italiana di diritto e procedura penale, $841-891$

Amodio, Ennio (1979a), Esperienze e prospettive della partecipazione popolare ai giudizi penali, Milano: Giuffrè

Amodio, Ennio (1979b), Giustizia popolare, garantismo e partecipazione, in: Amodio, EnNio (a cura di), I giudici senza toga, Milano: Giuffrè

162 Sul giusto processo cfr. fra gli altri Alessi (2001) 1 ss.; Amodio (2003d) 141 ss.; Ferrua (2000) 49 ss.; Ubertis (2002) 173 ss.; Illuminati (2008) 521 ss.; Pisani (2009); Riccio (2010) 268 ss.; ORLANDi (2011) 77.

163 Cfr. tra gli altri Kostoris (2011) 328 ss.; Davigo/Sisti (2012); Mura/Patrono (2011); OrLandi (2012c) $22 \mathrm{ss.}$ 
Amodio, Ennio (1984), Un nuovo modello accusatorio per la giustizia penale, in: Questione giustizia, 101-108

Amodio, Ennio (1988a), Clima inquisitorio e clima accusatorio, due prassi a confronto, in: La difesa penale, 20-29

Amodio, Ennio (1988b), Il processo penale statunitense e il nuovo processo penale italiano. Miti e realtà della giustizia americana, in: Amodio/Cherif BAssiouni (1988) 32-48

Amodio, Ennio (2003a), Processo penale, diritto europeo e common law. Dal rito inquisitorio al giusto processo, Milano: Giuffrè

Amodio, Ennio (2003b), Miti e realtà della giustizia nord americana. Il modello accusatorio statunitense e il codice di procedura penale del 1989, in: Amodio (2003a), 141-151

Amodio, EnNio (2003c), Disgregazione e recupero del sistema, in: Verso la riscoperta di un modello processuale: convegno in memoria di Antonino Galati. Atti del Convegno Caserta, 12-14 ottobre 2001, Milano: Giuffrè, 27-43

Amodio, Ennio (2003d), Giusto processo, procès equitable, e fair trial: la riscoperta del giusnaturalismo processuale, in: Amodio (2003a), 131-151

Amodio, Ennio, M. Cherif Bassiouni (1988) (a cura di), Il processo penale negli Stati uniti d'America, Milano: Giuffrè

Andrioli, Virgilio (1965), Appunti di procedura penale, Napoli: Morano

Baratta, Alessandro (1993), La violenza e la forza, Alcune riflessioni su mafia, corruzione, e il concetto di politica, in: Dei delitti e delle pene, 120-131

Battaglia, Achille (1954), Processo alla giustizia, Roma, Bari: Laterza

Battaglia, Achille (1988), Diritto e libertà Scritti e discorsi di un giurista militante. Editi e inediti 1944-1960, a cura di Armani, Giuseppe, Roma: Edizioni Archivio trimestrale

Bellavista, Girolamo (1956), La difesa nella istruzione penale, in: Studi sul processo penale II, 82-98

Beria D’Argentine, Adolfo (1994), La magistratura, in: Lo stato delle istituzioni italiane: problemi e prospettive. Atti del Convegno Roma, 30 giugno - 2 luglio 1993, Milano: Il Saggiatore, 442-445

Bertoni, Raffaele (2009), XX Congresso Perugia 1989, in: Cento anni di Associazione magistrati, Milano: IPSOA, 191-195

Bettiol, Giuseppe (1950), Su alcune caratteristiche giuridiche e politiche del processo penale, in: Scritti giuridici in onore di Francesco Carnelutti IV, Padova: Cedam, 121-134

Bettiol, Giuseppe (1966), Istituzioni di diritto e procedura penale, Milano: Giuffrè Calamandrei, Piero (1953), La crisi della giustizia, in: Calamandrei, Piero et al., La crisi del diritto, Padova: Cedam, 157-176

Capograssi, Giuseppe (1950), Giudizio, processo, scienza, verità, in: Opere, V, 57-68

Cappellini, Paolo (2010), "Inconscio inquisitorio" e regimi autoritari: un collegamento necessario?, in: Garlati (2010b), 23-42 
Cappellini, Paolo (2011), Un dialogo finalmente maturo, Prospettive storiche e ricostruttive sul processo penale come «diritto costituzionale applicato» nell'Italia repubblicana, in: Negri / Pifferi (2011), 5-11

Cappellini, Paolo et al. (a cura di) (2012), Enciclopedia italiana di scienze, lettere ed arti, ottava appendice, Il contributo italiano alla storia del pensiero, Diritto, Roma: Istituto della Enciclopedia Italiana Treccani

Caprioli, Francesco (2011), La disciplina delle prove penali nel codice del 1913: un modello «empiricamente reazionario», in: Corte d'assise 2, 507-530

Carnelutti, Francesco (1950a), Torniamo al giudizio, in: Carnelutti (1950b), 34-46

Carnelutti, Francesco (1950b), Questioni sul processo penale, Bologna: Zuffi

Carnelutti, Francesco (1962a), Premessa, in: De Luca, Giuseppe (a cura di), Primi problemi della riforma del processo penale, Milano: Giuffrè, 1-18

Carnelutti, Francesco (1962b), La malattia del processo penale, in: Rivista di diritto processuale civile, $1-8$

Carnelutti, Francesco (1964a), Introduzione, in: Carnelutti (1964b), 1-6

Carnelutti, Francesco (1964b), Verso la riforma del processo penale, Milano: Giuffrè

Carrara, Francesco (1878), Il diritto penale e la procedura penale, in: Opuscoli di diritto criminale, Prato: Canovetti, 17-33

Carrara, Francesco (1883), Pensieri sulla giuria, in: Carrara, Francesco, Reminescenze di cattedra e foro, Lucca: Canovetti, 357-442

Carrara, Francesco (2005), Programma del corso di diritto criminale. Del giudizio criminale, Bologna: Il Mulino

Cavina, Marco (2012a), Il mito del processo breve. Fra cultura giuridica dei giuristi e cultura giuridica dei non giuristi, in: Cavina (2012b), 7-10

Cavina, Marco (a cura di) (2012b), Il processo breve. L'aspirazione alla brevità del processo penale fra storia e attualità, Bologna: Patron

Chiavario, Mario (1984), Nuovo modello di giustizia penale e libertà personale dell'imputato, in: Politica del diritto, 427-446

Chiavario, Mario (1991a), Qualche sollecitazione per un confronto, in: Chiavario (1991b), 3-20

Chiavario, Mario (1991b) (a cura di), Il nuovo codice di procedura penale visto dall'estero, Milano: Giuffrè

Chiavario, Mario (1991c), «Cultura» italiana del processo penale, in: Studi in onore di Giuliano Vassalli. Evoluzione del diritto e della procedura penale (1945-1990), II, Milano: Giuffrè, 525-573

Chiavario, Mario (1995), L'azione penale tra diritto e politica, Padova: Cedam

Chiavario, Mario (1998), Garanzie individuali ed efficienza del processo, in: Il giusto processo, Milano: Giuffrè, 51-77

Chiodi, Giovanni (2011), Custodia preventiva e garanzie della libertà personale nel primo decennio repubblicano: il caso della scarcerazione automatica, in: Negri / Pifferi (2011), 233-269 
Cianferotti, Giulio (2008), Introduzione. Logica del processo, logica del giudizio ed opinione pubblica, in: Colao (2008), 15-27

Colao, Floriana et al. (2008), Processo penale e opinione pubblica in Italia tra Otto e Novecento, Bologna: Il Mulino

Colao, Floriana (2013a), Carrara, Francesco, in: Cortese, Ennio et al. (a cura di), Dizionario biografico dei giuristi italiani (XII-XX secolo), Bologna: Il Mulino, 463-466

Colao, Floriana (2013b), Giustizia e politica. Il processo penale nell'Italia repubblicana, Milano: Giuffrè

Conso, Giovanni (1969a), Nel XX anniversario dei Diritti dell'uomo: un bilancio sconfortante, in: Conso (1969b)

Conso, Giovanni (1969b), Costituzione e processo penale. Dodici anni di pagine sparse, 1956-1968, Milano: Giuffrè

Conso, Giovanni (1980), La libertà provvisoria a confronto con le esigenze di tutela della collettività, ovvero la 'legge Reale' tra politica e diritto, in: Giurisprudenza costituzionale, $470-478$

Conso, Giovanni (1993), L'ordinamento della giurisdizione nei suoi profili di maggiore attualità, in: Giurisdizione e cultura della legalità. Le regole del processo penale alla prova. Atti del Convegno promosso dalla Commissione giustizia della Camera dei Deputati, Roma: Camera de Deputati, 203-310

Conso, Giovanni et al. (1989), Il nuovo codice di procedura penale dalle leggi delega ai decreti delegati, Padova: Cedam

Conso, Giovanni, Giuseppe Riccio (1998) (a cura di), Codice di procedura penale, Torino: Utet

Cordero, Franco (1963), Tre studi sulle prove penali, Milano: Giuffrè

Cordero, Franco (1986), Guida alla procedura penale, Torino: Utet

Cordero, Franco (1997a), L'istruzione sommaria nel conflitto tra le due corti, in: Cordero (1997f), 11-33

Cordero, Franco (1997b), Stilus curiae (Analisi della sentenza penale), in: Cordero (1997f), 225-245

Cordero, Franco (1997c), Diatribe sul processo accusatorio, in: Cordero (1997f), $189-215$

Cordero, Franco (1997d), Strutture d'un codice, in: Cordero (1997f), 247-254

Cordero, Franco (1997e), Linee di un processo di parti, in: Cordero (1997f), 159-187

Cordero, Franco (1997f), Ideologie del processo penale con un'appendice, Roma: Università degli Studi La Sapienza

Costa, Pietro (1993), Un modello per un'analisi: la teoria del "garantismo" e la comprensione storico giuridica della "modernità" penalistica, in: Gianformaggio, Letizia (a cura di), Le ragioni del garantismo, discutendo con Luigi Ferrajoli, Torino: Giappichelli, 11-21

Costa, Pietro (2011), Il modello accusatorio in Italia tra attuazione della Costituzione e mutamento di paradigma, in: Negri / PIfFerI (2011), 151-160 
Costa, Pietro (2012), Di cosa fa storia la storia della giustizia? Qualche considerazione di metodo, in: Lacchè / Meccarelli (2012), 17-43

Cottino, Amedeo, Claudio Sarzotti (a cura di) (1995), Diritto, uguaglianza e giustizia penale, Milano: L'Harmattan

Criteri direttivi per una riforma del processo penale (1965), Milano: Giuffrè

Da Passano, Mario (1989), Il giurì, «compagno indispensabile, necessario, fatale, della libertà", in: Movimento operaio e socialista, 257-273

Damaska, Mirjan R. (1991), I volti della giustizia e del potere. Analisi comparatistica del processo, Bologna: Il Mulino

Damaska, Mirjan R. (2003), Il diritto delle prove alla deriva, Bologna: Il Mulino

D'Aniello, Mario (a cura di) (1955), Innovazioni al codice di procedura penale con tutte le norme di attuazione ..., Milano: Giuffrè

Davigo, Piercamillo, Leo Sisti (2012), Processo all'italiana, Roma, Bari: Laterza

De Francesco, Giovannangelo (2012), Francesco Carrara, in: Cappellini (2012), 357-361

De Leo, Francesco (1984), Quale processo per quale giurisdizione, in: Questione giustizia, 148-163

Dezza, Ettore (2010), «Justice n'est proprement autre chose que formalité», in: Garlati (2010b), 167-186

Dezza, Ettore (2011), Accusa e inquisizione nell'esperienza italiana contemporanea, in: Negri / Pifferi (2011), 101-116

Discorso del procuratore generale Silvio Longhi per inaugurazione dell'anno giudiziario alla Corte di Cassazione (1931), in: Rivista penale, 1-23

Discorso di S. E. Grandi (1939), in: La riforma fascista dei codici, Roma: Tipografia delle Mantellate

Ferrajoli, Luigi (1989), Diritto e ragione. Teoria del garantismo penale, Roma, Bari: Laterza

Ferri, E. (a cura di) (1985), Un nuovo processo penale per una società migliore, in: Il nuovo contratto sociale. Dieci anni di attività del centro lunigianese di studi giuridici. Presentazione di S. Pertini, Milano: Sugarco

Ferri, Enrico (1883), La scuola positiva di diritto criminale, Siena: Torrini

Ferrua, Paolo (1987), I maxiprocessi e la legge 17 Febbraio 1987, in: Cassazione penale, $1662-1179$

Ferrua, Paolo (1993), Contraddittorio e verità nel processo penale, in: Gianformaggio, Letizia (a cura di), Le ragioni del garantismo, discutendo con Luigi Ferrajoli, Torino: Giappichelli, 212-262

Ferrua, Paolo (2000), Il processo penale dopo la riforma dell'art. 111 della Costituzione, in: Questione giustizia, 49-76

Fisher, George (2003), Plea Bargaing's Triumph. A History of Plea Bargaing in America, Standford: Standford University Press

Fortuna, Ennio (1984), Modello ideale e modello reale nel sistema processuale penale, in: Cassazione penale, 2322-2342

Foucault, Michel (1994), La verità e le forme guridiche, Napoli: Esi

Galli, Carlo (2009), Tangentopoli, crisi di legalità, crisi di legittimità, in: Ius 17, 1 
Gambini Musso, Rosanna (1985), Il plea bargaing tra common law e civil law, Milano: Giuffrè

Garapon, Antoine (2007), Del giudicare. Saggio sul rituale giudiziario, Milano: Cortina Raffaello

Garapon, Antoine, Denis Salas (1998), La Repubblica penale, Milano: Oche del Campidoglio

Garlati, Loredana (2010a), Le ragioni di un incontro, in: Garlati (2010b), 1-5

GARLATI, LOREDANA (a cura di) (2010b), L'inconscio inquisitorio. L'eredità del codice Rocco nella cultura processualpenalistica italiana, Milano: Giuffrè

Garofalo, Raffaele (1881), Criminologia. Studio sul delitto e sulla teoria della repressione, Torino: Utet

Garofalo, Raffaele (1892), La detenzione preventiva, in: La scuola positiva, 199-206

Giarda, Angelo (1993), Intervento, in: Giurisdizione e cultura della legalità. Le regole del processo penale alla prova. Atti del Convegno promosso dalla Commissione giustizia della Camera dei Deputati, Roma: Camera de Deputati, 60-66

Giostra, Glauco (1995), Il processo penale «contro» la criminalità organizzata: rischi di strumentalizzazione, in: Giostra, Glauco, Gaetano Insolera (a cura di), Lotta alla criminalità organizzata: gli strumenti normativi, Milano: Giuffrè, $155-163$

Gismondi, Antonio (1914), Codice di procedura penale annotato in base alle Relazioni ministeriali e parlamentari e ai risultati della dottrina e della giurisprudenza, Roma: Atheneum

Giurisdizione e cultura della legalità: le regole del processo penale alla prova (1993), Roma: Camera dei Deputati

Graziano, Silvestro (1912), L'intervento della difesa nell'istruttoria penale, Bologna: Zanichelli

Grevi, Vittorio (1997), Garanzie individuali ed esigenze di difesa sociale nel processo penale, in: Lanfranchi, Lucio (a cura di), Garanzie costituzionali e diritti fondamentali, Roma: Istituto dell'Enciclopedia italiana, 255-286

Grevi, Vittorio (2003), Alla ricerca di un processo penale "giusto", Milano: Giuffrè

Grossi, Paolo (2012), Introduzione al Novecento giuridico, Roma, Bari: Laterza

Guarnieri, Carlo (2011), La giustizia in Italia, Bologna: Il Mulino

Illuminati, Giulio (2008), Costituzione e processo penale, in: Giurisprudenza italiana, 521-528

Incampo, Antonio, Vincenzo Garofoli (a cura di) (2012), Verità e processo penale, Milano: Giuffrè

Intervento (1965), in: Criteri direttivi per una riforma del processo penale, Milano: Giuffrè, 186-193

Kagan, Robert A. (2001), Adversarial legalism. The american way of Law, Cambridge, London: Harvard University Press

Kagan, Robert A. (2007), American and European Ways of Law: six entrenched differences, in: European Ways of Law, Oxford: Hart, 41-70 
Kagan, Robert A. (2009), La giustizia americana. Come il contraddittorio si fa diritto, Bologna: Il Mulino

Kirchheimer, Otto (2002), Giustizia politica, a cura di Racinaro, Roberto, Macerata: Liberi libri

Kostoris, Roberto E. (2011), Modello accusatorio, cultura inquisitoria, scenari europei tra presente e futuro del processo penale, in: NeGRI/PIfFERI (2011), 323-329

La Costituzione della Repubblica italiana nei lavori preparatori dell'Assemblea Costituente, VI (1970), Roma: Camera dei Deputati

La testimonianza di un protagonista. Intervista a Mario Chiavario (2011), in: Negri / Pifferi (2011), 421-437

La vera storia d'Italia. Interrogatori, testimonianze, riscontri, analisi. Giancarlo Caselli e i suoi sostituti ricostruiscono gli ultimi vent'anni di storia italiana (1995), Napoli: Pironti

LACCHÈ, Luigi (1990), La giustizia per i galantuomini. Ordine e libertà nell'Italia liberale: il dibattito sul carcere preventivo (1863-1913), Milano: Giuffrè

LAcChè, Luigi (2006), Una letteratura alla moda, Opinione pubblica, "processi infiniti” e pubblicità in Italia tra Otto e Novecento, in: Miletti, Marco Nicola (a cura di), Riti tecniche interessi. Il processo penale tra Otto e Novecento, Milano: Giuffrè, 459-513

LAcChè, Luigi (2007a), La penalistica costituzionale e il "liberalismo giuridico". Problemi e immagini della legalità nella riflessione di Francesco Carrara, in: Quaderni fiorentini per la storia del pensiero giuridico moderno, 36, 661-695

LACCHÈ, Luigi (2007b), L'«opinione pubblica saggiamente rappresentata». Giurie e Corti d'assise nei processi celebri tra Otto e Novecento, in: Marchetti, Paolo (a cura di), Inchiesta penale e pregiudizio. Una riflessione interdisciplinare, Napoli: Esi, 89-147

LACChè, Luigi (2008), Un luogo «costituzionale» dell'identità giudiziaria nazionale: la Corte d'assise e l'opinione pubblica, in: Colao (2008), 77-120

LACchÈ, Luigi (2010), «Sistemare il terreno e sgombrare le macerie». Gli anni della «Costituzione provvisoria»: alle origini del discorso sulla riforma della legislazione e del codice di procedura penale (1943-1947), in: Garlati (2010b), 271-304

LAcchè, Luigi (2011), I diritti della storia e la storia dei diritti. Riflettendo sul processo nell'Italia repubblicana, in: Negri / Pifferi (2011), 87-100

Lacchè, Luigi, Massimo Meccarelli (a cura di) (2012), Storia della giustizia e storia del diritto, Macerata: Eum

Lacchè, Luigi, Massimo Meccarelli (2012a), Introduzione. Storia del diritto come storia della giustizia: materiali per una riflessione metodologica, in: LACCHè / MecCARELli (2012), 7-16

Lacchè, Luigi, Monica Stronati (a cura di) (2012), Una tribuna per le scienze criminali. La "cultura” delle Riviste nel dibattito penalistico tra Otto e Novecento, Macerata: Eum 
Langer, Máximo (2004), From Legal Transplants to Legal Translations: The Globalization of Plea Bargaing and the Americanization Thesis in Criminal Procedure, in: Harvard International Law Journal, 1-63

Latini, Carlotta (2012), Luigi Lucchini, in: Cappellini (2012), 362-365

Leone, Giovanni (1955), Lineamenti di diritto processuale penale, Napoli: Jovene

Leone, Giovanni (1964a), Linee generali di una riforma nel processo penale, in: LEONE (1964b), 33-62

Leone, Giovanni (1964b), Intorno alla riforma del codice di procedura penale. Raccolta di scritti. Milano: Giuffrè

Leone, Giovanni (1964c), Problemi contingenti di riforma, in: Leone (1964b), 113-161

Leone, Giovanni (1990a), Interventi e studi sul processo penale, Napoli: Jovene

Leone, Giovanni (1990b), Rilievi critici sulla legge delega del codice di procedura penale, in: LeONE (1990a), 141-149

Lombardi, Giovanni (1914), Pregi e difetti della nuova procedura, in: Rivista di diritto e procedura penale, 321-332

Lombroso, Cesare (1876), L'uomo delinquente, Torino: Utet

Longhi, Silvio (1925), La nuova disciplina sulla stampa, in: Dizionario penale, 200-209

Lucchini, Luigi (1873), Pubblicità, oralità e contraddittorio nella istruttoria del processo penale. Appunti critici, Verona: Tip. M. Dal Ben

Lucchini, Luigi (1913), Il nuovo codice di procedura penale. Impressioni e appunti, in: Rivista penale, 681-689

Malinverni, Alessandro (1972), Lineamenti di storia del processo penale, Torino: Giappichelli

Manes, Vittorio (2009), Il fenomeno Tangentopoli, la legalità della politica e la legittimità dell'intervento penale, in: Ius 17,1, 243-254

Manzini, Vincenzo (1931a), Trattato di diritto processuale penale secondo il nuovo codice, Torino: Utet

Manzini, Vincenzo (1931b), Istituzioni di diritto processuale penale, Padova: Cedam

Manzini, Vincenzo (1956), Trattato di diritto penale processuale, Torino: Utet

Marchetti, Paolo (2009), Le "sentinelle del male". L'invenzione ottocentesca del criminale nemico della società tra naturalismo giuridico e normativismo psichiatrico, in: Quaderni fiorentini per la storia del pensiero giuridico moderno 38, II, 1009-1080

Marchetti, Paolo (2012), Cesare Lombroso e l'«Archivio di psichiatria», in: Lacchè / Stronati (2012), 69-96

Marcolini, Stefano (2005), Il patteggiamento nel sistema della giustizia penale negoziata: l'accertamento della responsabilità nell'applicazione della pena su richiesta delle parti tra ricerca di efficienza ed esigenze di giustizia, Milano: Giuffrè

Marmo, Marcella (2003), "Processi indiziari non se ne dovrebbero mai fare», in: Marmo, Marcella, Luigi Musella, La costruzione della verità giudiziaria, Napoli: Clio press, 101-117 
Meccarelli, Massimo (2008), «Due lati di una stessa figura». Questioni di fatto e di diritto tra corte di assise e Cassazione nel dibattito dottrinale verso il codice di procedura penale del 1913, in: Colao (2008), 163-194

Meccarelli, Massimo (2012), Ludovico Mortara, in: Cappellini (2012), 459-462

Meniconi, Antonella (2006), La maschia avvocatura. Istituzioni e professione forense in epoca fascista (1922-1943), Bologna: Il Mulino

Meniconi, Antonella (2012), Storia della magistratura italiana, Bologna: Il Mulino

Miletti, Marco Nicola (2003), Un processo per la terza Italia. Il codice di procedura penale del 1913, I, L'attesa, Milano: Giuffrè

Miletti, Marco Nicola (2007), Ombre d'inquisizione. L'intervento della difesa nell'istruttoria penale italiana, in: Quaderni fiorentini per la storia del pensiero giuridico moderno XXXVI, 901-955

Miletti, Marco Nicola (2008), «Uno zelo invadente». Il rifiuto della pubblicità istruttoria nel codice di procedura penale del 1930, in: Colao (2008), 227-262

Miletti, Marco Nicola (2010), La scienza nel codice. Il diritto processuale penale nell'Italia fascista, in: Garlati (2010b), 57-107

Miletti, Marco Nicola (2011), Un vestito per Cenerentola: l'identità del diritto processuale penale all'alba della Repubblica, in: Negri/Pifferi (2011), 363-393

Miletti, Marco Nicola (2012), La giustizia penale, in: Cappellini (2012), 467-474

Miletti, Marco Nicola (2013a), Lucchini, Luigi, in: Cortese, Ennio et al. (a cura di), Dizionario biografico dei giuristi italiani (XII-XX secolo), Bologna: Il Mulino, 1207-1211

Miletti, Marco Nicola (2013b), Pessina, Enrico, in: Cortese, Ennio et al. (a cura di), Dizionario biografico dei giuristi italiani (XII-XX secolo), Bologna: Il Mulino, 1554-1558

Miletti, Marco Nicola (2013c), Longhi, Silvio, in: Cortese, Ennio et al. (a cura di), Dizionario biografico dei giuristi italiani (XII-XX secolo), Bologna: Il Mulino, 1193-1195

Moccia, Sergio (2000), La perenne emergenza. Tendenze autoritarie nel sistema penale, Napoli: Esi

Mortara, Lodovico (1914), I delitti di sangue in Italia, Roma

Mortara, Lodovico (1992), La giustizia nello Stato democratico (1912), in: Mortara, Lodovico, Lo Stato moderno e la giustizia, Napoli: Esi, 188-199

Mura, Antonello, Antonio Patrono (2011), La giustizia penale in Italia. Un processo da sbloccare. La lezione americana, Padova: Cedam

Natale, Michele (2013), Una breve riflessione sul codice del 1913. Azione penale, pubblico ministero e giudice istruttore tra modello misto e suggestioni accusatorie, in: Historia et Ius 3 (available at: http://www.historiaetius.eu/uploads/ 5/9/4/8/5948821/natale_1913.pdf)

Negri, Daniele, Michele Pifferi (a cura di) (2011), Diritti individuali e processo penale nell'Italia repubblicana, Milano: Giuffrè

Neppi Modona, Guido (1984), I meccanismi processuali differenziati, in: Cassazione penale, $423-448$ 
Neppi Modona, Guido (1987), Commento all'art. 112, in: Branca, Giuseppe (a cura di), Commentario della Costituzione. La magistratura, IV, Bologna, Roma: Il Mulino, 52-68

Neppi Modona, Guido (1991), Processo accusatorio e tradizione giuridica continentale, in: Chiavario, Mario (1991b)

Neppi Modona, Guido (2000), La parabola dei riti alternativi tra interventi della Corte costituzionale e modifiche legislative, in: Studi in onore di Gian Domenico Pisapia, II, Milano: Giuffrè, 447-475

Neppi Modona, Guido (2007), Diritto e giustizia nel periodo fascista, in: Lacchè, Luigi et al. (a cura di), Penale, giustizia, potere. Per ricordare Mario Sbriccoli, Macerata: Eum, 341-378

Neppi Modona, Guido (2010), Quali giudici per quale giustizia nel ventennio fascista, in: Garlati (2010b), 209-224

Nobili, Massimo (1998), Scenari e trasformazioni del processo penale, Padova: Cedam

Nobılı, Massımo (2009), L'immoralità necessaria. Citazioni e percorsi nei mondi della giustizia, Bologna: Il Mulino

Nuvolone, Pietro (1946), Il momento penale, in: Archivio penale, 263-278

Nuvolone, Pietro (1964), Legalità, giustizia e difesa sociale, in: Rivista italiana di diritto e procedura penale, $17-38$

Nuvolone, Pietro (1965), L'istruzione penale, in: Criteri direttivi per una riforma del processo penale, Milano: Giuffrè, 84-96

Orlandi, Renzo (2006), Rito penale e salvaguardia dei galantuomini, in: Criminalia, 293-309

Orlandi, Renzo (2010), La riforma fascista delle corti d'assise, in: Garlati (2010b), 225-240

Orlandi, Renzo (2011), Diritti individuali e processo penale nell'Italia repubblicana, in: Negri / Pifferi (2011), Milano: Giuffrè, 51-83

Orlandi, Renzo (2012a), L'utilità dello sguardo storico in epoca di grandi svolte, in: Tavilla, Elio (a cura di), La giustizia criminale: premesse per un terreno di indagine comune, Bologna: Patron, 79-84

Orlandi, Renzo (2012b), La giustizia penale, in: Cappellini (2012), 675-682

Orlandi, Renzo (2012c), L'insostenibile lunghezza del processo penale e le sorti progressive dei riti speciali, in: Rivista di diritto processuale, 22-37

Perchiunno, Vincenzo (1996), Percorsi di procedura penale. Dal garantismo inquisitorio all'accusatorio non garantito, Milano: Giuffrè

Pessina, Enrico (1904), La difesa dell'imputato nella istruzione del processo, Napoli: Eugenio Marghieri

Picardi, Nicola (2013), Mortara, Lodovico, in: Cortese Ennio et al. (a cura di), Dizionario biografico dei giuristi italiani (XII-XX secolo), Bologna: Il Mulino, 1383-1386

Pifferi, Michele (2013a), Alla ricerca del «genio italico». Tradizione e progetti nella penalistica post-unitaria, in: Cazzetta, Giovanni (a cura di), Retoriche dei giuristi e costruzione dell'identità nazionale, Bologna: Il Mulino, 257-295 
Pifferi, Michele (2013b), L'individualizzazione della pena. Difesa sociale e crisi della legalità penale tra Otto e Novecento, Milano: Giuffrè

Pisani, Mario (1995), La stagione delle «mani pulite». Notizie dall'Italia, in: L'Indice penale, 167-168

Pisani, Mario (1998), Italian style. Figure e forme del nuovo processo penale, Padova: Cedam

Pisani, Mario (1999), Il processo penale nel dedalo delle riforme, in: L'Indice Penale, 545-562

Pisani, Mario (2002a), Il lungo cammino del codice del 1988, in: Pisani, Mario (2002b), Manuale di procedura penale, Bologna: Monduzzi, 1-37

Pisani, Mario (2009), Riflessioni sul «giusto processo penale», in: Istituzioni pubbliche e garanzie del cittadino, Milano: Giuffrè, 139-147

Pisapia, Gian Domenico (1989a), Un nuovo modello di processo, in: Legislazione penale, $77-88$

Pisapia, Gian Domenico (a cura di) (1989b), Il nuovo codice di procedura penale dalle indagini preliminari al dibattimento, Milano: Giuffrè

Pisapia, Gian Domenico (1993), Ma Perry Mason non abitò mai qui. Finzione e realtà del processo accusatorio, «Il Sole 24 ore», 15 Settembre 1993

Ramat, Marco (1985), Il «maxiprocesso», in: Questione giustizia, 259-273

Relazione del Guardasigilli al progetto preliminare, in Codice di procedura penale illustrato con i lavori preparatori (1930), Roma: Tip. Le Mantellate

Resta, Eligio (2004), La verità e il processo, in: Politica del diritto, 369-381

Riccio, Giuseppe (2010), La procedura penale tra storia e politica, Napoli: Esi

Rocco, Alfredo (1925), Riforma del codice penale, del codice di procedura penale e delle leggi sull'ordinamento giudiziario, Roma: Tip. del Senato

Rocco, Alfredo (1930), Relazione al progetto definitivo, in: Lavori preparatori del codice penale e di procedura penale, X, Roma: Tip. Le Mantellate, 7-31

Rocco, Alfredo (1931a), La nuova corte d'Assise, in: Rivista penale, 501-510

Rocco, Alfredo (1931b), Prefazione, in: Manzini, Vincenzo (1931a), IX-X

Ruggieri, Francesca (2012), Il caso della procedura penale, in: Cassazione penale, 1131-1158

Satta, Salvatore (1994), Il mistero del processo, Milano: Adelphi

Sbriccoli, Mario (2009a), La "Rivista Penale" di Luigi Lucchini (1874-1900), in: SBricCOLI (2009c), 903-980

Sbriccoli, Mario (2009b), Le mani nella pasta e gli occhi al cielo. La penalistica italiana negli anni del fascismo, in: SBriccoli (2009c), 1001-1134

Sbriccoli, Mario (2009c), Storia del diritto penale Storia del diritto penale e della giustizia. Scritti editi e inediti (1972-2007), Milano: Giuffrè

Sbriccoli, Mario (2009d), Caratteri originari e tratti permanenti nel sistema penale italiano (1860-1950), in: SBriccoli (2009c), 591-670

Sbriccoli, Mario (2009e), Codificazione civile e penale, in: Sbriccoli (2009c), 981-991 
Seminara, Sergio (2012), Necessità della storia, in: Tavilla, Elio (a cura di), La giustizia criminale: premesse per un terreno di indagine comune, Bologna: Patron, $85-88$

Sotis, Carlo (2007), Il diritto senza codice. Uno studio sul sistema penale europeo vigente, Milano: Giuffrè

Storti, Claudia (1998), Giuria penale ed errore giudiziario. Questioni e proposte di riforma alle soglie della promulgazione del codice del 1913, in: Gouron, ANDré et al. (a cura di), Error iudicis. Juristische Wahrheit und justizieller Irrtum, Frankfurt am Main: Klostermann

Storti, Claudia (2008), Incredulità e «malsana curiosità» dell'opinione pubblica: la logica dell'istruttoria tra politica legislativa e giurisprudenza di Cassazione (1898-1930), in: Colao (2008), 121-173

Storti, Claudia (2011a), "Un mezzo artificiosissimo di governo per ottenere con inganno e per vie coperte ciò che apertamente non si potrebbe ordinare”. Le circolari dei ministri di giustizia sul processo penale tra unificazione e fascismo, in: Colao, Floriana et al. (a cura di), Perpetue appendici e codicilli alle leggi italiane. Le circolari ministeriali, il potere regolamentare e la politica del diritto in Italia tra Otto e Novecento, Macerata: Eum, 577-627

Storti, Claudia (2011b), Magistratura e il diritto di difesa nell'istruzione penale, in: Negri / Pifferi (2011), 179-198

Stronati, Monica (2012a), «Un'oncia di pratica»: Enrico Ferri e gli “esordi” della rivista «La Scuola Positiva», in: Lacchè / Stronati (2012), 97-117

Stronati, Monica (2012b), Enrico Ferri, in: Cappellini (2012), 371-375

Sulle attribuzioni degli organi del Pubblico Ministero (Circolare 6 Luglio 1931 del Ministro della Giustizia) (1931), in: La scuola penale unitaria

Taruffo, Michele (2009), La semplice verità. Il giudice e la costruzione dei fatti, Roma, Bari: Laterza

Ubertis, Giulio (2002), Diritti fondamentali e processo penale, in: Mazzarese, Tecla (a cura di) Neocostituzionalismo e tutela (sovra)nazionale dei diritti fondamentali, Torino: Giappichelli, 173-188

Vacca, Guglielmo (1912), Commento al codice di procedura penale, I, Torino: Utet

Vassalli, Giuliano (1997), Il codice di procedura penale del 1988: suoi contenuti e successive vicende, in: Vassalli, Giuliano, Scritti giuridici, III, Milano: Giuffrè, 805-821

Vassalli, Giuliano (2010), Introduzione, in: Garlati, Loredana (a cura di), L'inconscio inquisitorio. L'eredità del codice Rocco nella cultura processualpenalistica italiana, Milano: Giuffrè, 9-22

Zagrebelsky, Vladimiro (1998), La magistratura ordinaria dalla Costituzione ad oggi, in: Violante, Luciano (a cura di), Storia d'Italia, Annali 14, Diritto, legge, giustizia, Torino: Einaudi, 714-790 



\section{Beyond the Lighthouse. Sicily and the 'Sicilies': Institutional Readings of a Borderland}

to Vincenzo Consolo

\section{The trident of Poseidon}

Originally there was Poseidon enosichton, shaker of the Earth: the trident of the sea god crush the continuity, the telluric gash that separates Sicily from the continent, the waters that flow in the Strait which will be called from Messina separate the island forever from the mainland. Thus the Trinacria borns, the three-cornered island, separated from the Italian peninsula by a border-not border, the sea of Homer, legendary rule of the monsters Scylla and Charybdis. ${ }^{1}$

An island, as they believed, has definite borders; its coasts marke the exact boundary; the sea demarcates its extent.

But Sicily escapes this cliché: for centuries the name 'Sicily' indicated a kingdom that stretched far beyond of the limit assigned by the trident of Poseidon. The events of the Middle Ages saw the creation of a Tema Byzantine of Sicily which also included the southern area of Calabria, the Brutium; then the Arab invasion broke this continuity; and the creation of the Norman kingdom identified as Kingdom of Sicily also the southern part of Italy, to the ends of Patrimonium Sancti Petri.

Subsequent events of the Sicilian Vespers know again a kingdom of Sicily coincident with the island, while also the part of the kingdom remained in the hands of Anjou was titled in the same way, to claim the legitimacy of the government and the will to recover the island (the peace of Caltabellotta sanctioned, moreover, a new name for the island, that of the kingdom of Trinacria). Stories of a war that lasted a century ended with the Aragonese conquest of southern Italy: the kingdom of Sicily joined himself in the two

1 This striking image in ArIcò (1999). 
integral parts, continental and insular, under the sceptre of Alfonso V of Aragon.

In these circumstances, the two areas of 'Sicily' are distinguished taking like reference point the ancient lighthouse of Messina, which rose on Pelorus, where once rose up the temple of Poseidon enosichton and the pharos. ${ }^{2}$ Since the triumph of Alfonso, celebrated over the Maschio angioino, you can distinguish a Sicily on this side of the Lighthouse - the kingdom of Naples - from a Sicily beyond the Lighthouse - Sicily itself. The strange thing is that the boundary between the two kingdoms, in a vision evidently from Neaples, has been identified in a symbolic building that stood in Sicily, almost by ascribing the Strait to the continental part of the kingdom.

Two Sicilies, separated / united by Scylla and Charybdis, with two capitals, then two viceroy in Palermo and Naples, different institutions, for example a parliament in the island who don't exist in the continent. Two kingdoms that will be reunited by Bourbon's reformism with the Legge fondamentale of 1816, which created truely the Kingdom of the Two Sicilies, with its capital Naples, and only a faint appearance of autonomy for the island. Here begins a phase of endemic revolution, which saws the Sicilians who did not accept these innovations and fought in the name of the independence of the ancient kingdom, the defense of the island's Constitution of 1812 , and the restoration of parliamentary prerogatives. The government responded with an hard repression, which eliminates the residual aspirations for autonomy. Even the Neapolitan 'revolutionary' parliament of 1820 came to alter the geography, denying the existence of the Strait of Messina: the meeting between the Ionian and the Tyrrhenian Sea was indicated with the definition of 'River Lighthouse' to avoid 'naturalistic' pretexts to the division of the two kingdoms, ${ }^{3}$ nearly to put down the Sicilian separatism with a mere terminological operation, which degraded Scylla to a river nymph.

Another unique aspect of the border terraqueous of Trinacria is that at least since the time of the Byzantine Tema, Sicily has extended its jurisdiction to include the so-called smaller islands. The kingdom of the islands added the Aeolian Islands, the Egadi Islands, the Pelagie, the Maltese Islands, Usti$\mathrm{ca}$, and Pantelleria. Lands of restless volcanoes, from the giant Etna to Vulcano and Stromboli, to include in a sort of institutional farce the Ferdinandea

2 On the temple and the lighthouse Aricò (1999).

3 Palmieri (1847) 407. 
island, the mouth of a submerged volcano, appeared thanks to an eruption in 1831 and swallowed up by the waves the following year, and that was its very short existence. This island was contested by England, France and Two Sicilies, from time to time then was called Graham, Julia, Ferdinandea, Corrao (from the captain who discovered it). ${ }^{4}$ Still faint borders, land and sea which are merged in the islands of the fire.

\section{Valli, districts, comarche}

Sicily, an island: she is demarcated by definite boundary by antonomasia: its borders are recognizable without fail. In this way the idea of the possibility of a moving border, of a strip of uncertainty, the need innate in man to expand his sphere of rule and power, finding no outlet over the cliffs and beaches, it is retreated inward towards the interior of Sicily. Through its long history, there has been a continuous definition and re-definition of the administrative subdivisions of the island, particularly manifest since the Middle Ages. If you talk in Sicily of colonization, it has indicated the foundation of rural villages in the feudal lands which occurred especially among sixteenth and seventeenth centuries.

The best known is the division into Valli, which according to tradition there were three. These took their names from the main cities of the area at the time of the Arabian conquest: the Val di Noto, Val Demone (which remind the name of the ancient and lost town of Demenna), and the Val di Mazara. The term Val - which is a masculine - derives from Walayah (district, prefecture) or Wilayah (district government, the jurisdiction of a judge, the Wali). The Norman chancery adapted to Latin the Arabic word, which became the term Val. $^{5}$

In Norman time the valli were probably four, coinciding with the provincial justicers districts: Demenna, Noto, Castrogiovanni, Mazara. Frederick II reduced them to two (citra and ultra Salso river), then dividing them into six smaller districts: Noto, Demone, Mazara, Agrigento, Castrogiovanni, Milazzo. In fact the ancient records confirm the existence of the six valli

4 Today, the top of the volcano is located about 8 meters below sea level. On the Island Ferdinandea cf. Mazzarella (1984); Mirabella (2015).

5 Kislinger (2005) 9-20. 
which coincide with the districts of justicers: during the fourteenth century these areas suffered momentous changes, related to the upheaval of the era of the four vicars. The fifteenth century saw again zoning into three valli, but the justicers of the provinces had much reduced their role. ${ }^{6}$

Also inside the valli there were further boundaries: first, those of the dioceses, not always and not necessarily coinciding with the institutional limits; then the feuds and other subdivisions. A striking example is that of the Val di Noto, which corresponds to the south-eastern Sicily. In the Aragonese age, a major part of the Vallo was the dotarium of queens of Sicily, the Chamber of the queens, with Syracuse as its capital, and that included other major towns like Lentini, Vizzini, Mineo. In the Val di Noto was also located the largest and most conspicuous feud of the island, the County of Modica, which was arrogantly defined in the modern age as one 'state into the state', including, in addition to the eponymous capital, the town of Ragusa and Scicli as well as numerous smaller towns. Most of the Val di Noto was placed under the jurisdiction of the bishop of Syracuse, while the northern part fell into that of the bishop of Catania. As you can see an impressive mix of powers, forces, courts, the cause of frequent and interminable disputes.

The division into three Valli persisted across all the 'long Middle Ages', to the important innovations sanctioned in the Constitution of Sicily of 1812, which divided the island into 23 districts or comarche: Alcamo, Bivona, Caltagirone, Caltanissetta, Castroreale, Catania, Cefalù, Corleone, Girgenti, Mazara, Messina, Mistretta, Modica, Nicosia, Noto, Palermo, Patti, Piazza, Sciacca, Siracusa, Termini, Terranova and Trapani. The 'rational' criteria identified by the parliament in order to delimit the boundaries of the new institutions were 'natural' (rivers, mountains and valleys), combined with the possibility of effective control of the land by the companies of 'capitani d'armi' (once again 'geographical' and 'legal' criteria are closely intertwined); finally, the capitals of the comarche had to be identified in their main towns.

After, the reforms of the administrative monarchy in 1817 created seven valli, corresponding to the new provinces, the Intendenze: Palermo, Messina, Catania, Siracusa, Girgenti, Caltanissetta, Trapani. This was to reduce the

6 Marrone (2011). 
role of the ancient capital of the lost kingdom, Palermo; the government wanted to create close agreements with local authorities to 'punish' the parliamentary aristocracy that had forced the king to swear to the Constitution of 1812. The 'War of Sicily' of 1820-21 did not succeed in restoring the independence of the island; the place of the three ancient Valli was token over definitively by the seven minor Valli, this time inflected at feminine and not at masculine, further subdivided into sottintendenze.

The unity of Italy saw the elimination of the intendenze, replaced by the provinces governed by the prefects. But nothing changed, because the seven Valli were unchanged: they only changed in the name; the only remarkable novelty was the return of the provincial administration in Siracusa, which had been moved to Noto to punish Siracusa after the bloody revolt of 1837 . Also the interior articulations only changed the name from sottointendenze to sottoprefetture.

This division remained unchanged until 1927, when the fascist government created two new provinces: Enna, the ancient Castrogiovanni, who supplanted the districts of Nicosia (dismembered from Catania) and Piazza (removed from Caltanissetta), where, however, remained hinged dioceses; and Ragusa, which replaced the ancient capital of the County, Modica, in the leadership of the iblea land (and where, instead, in 1950, a new bishop was located). The same year the sottoprefetture were suppressed, with the memory of districts of comarche of 1812.

\section{Sicily/Sicilies}

Di qua del faro. This side of the Lighthouse. Now we turn over to the perspective with which Sicily has become an area beyond the mainland, as in the title of this paper, and we reverse the usual view, following the provocative title of the homonymous book by Vincenzo Consolo, aimed at recovering the centrality of the Mediterranean island. ${ }^{7}$

The Trinacria, the three-cornered island. According to an ancient tale each corner is based on a column: one of these, deeply damaged, since the time of the emperor Frederick II would be supported by the legendary Colapesce -

7 Consolo (1999). 
for the people of Messina the column is that under the Cape Pelorus; for the inhabitants of Catania would be under Etna, and it is worn-out by the flames of the volcano; for still others would be under Cape Passero, and its failure would have caused the terrible earthquake that devastated the Val di Noto in 1693. Three corners, three columns, three valli. Already in its mythic dimension Sicily includes several Sicilies.

This perception finds one of the earliest and most profound expressions in Il Gattopardo by Tomasi di Lampedusa, in the famous dialogue between Don Fabrizio Salina and Chevalley, when the prince says:

«ho detto i Siciliani, avrei dovuto aggiungere la Sicilia, l'ambiente, il clima, il paesaggio. Queste sono le forze che insieme e forse più che le dominazioni estranee e gl'incongrui stupri hanno formato l'animo: questo paesaggio che ignora le vie di mezzo fra la mollezza lasciva e l'asprezza dannata; che non è mai meschino, terra terra, distensivo, umano, come dovrebbe essere un paese fatto per la dimora di esseri razionali; questo paese che a poche miglia di distanza ha l'inferno attorno a Randazzo e la bellezza della baia di Taormina, ambedue fuor di misura, quindi pericolosi». ${ }^{8}$

This topic finds theorizing in the works of Leonardo Sciascia, Gesualdo Bufalino, Vincenzo Consolo, who have at lenght discussed, they offer a very high contribution of reflection on the human condition and social development of the inhabitants of the island. Perhaps the most condensed expression of this vision of a land that includes different worlds is that of Bufalino in Isola plurale (now in Cere perse):

«[...] Vi è una Sicilia 'babba', cioè mite, fino a sembrare stupida; una Sicilia 'sperta', cioè furba, dedita alle più utilitarie pratiche della violenza e della frode. Vi è una Sicilia pigra, una frenetica; una che si estenua nell'angoscia della roba, una che recita la vita come un copione di carnevale; una, infine, che si sporge da un crinale di vento in un accesso di abbagliato delirio [...] Tante Sicilie, perché? Perché la Sicilia ha avuto la sorte di ritrovarsi a far da cerniera nei secoli fra la grande cultura occidentale e le tentazioni del deserto e del sole, tra la ragione e la magia, le temperie del sentimento e le canicole della passione. [...] Non è tutto, vi sono altre Sicilie, non si finirà mai di contarle».?

In the words of the great author of Comiso it's possible to perceive the role of a mythical space and time who rise and sentence at the same time the islander at a distinctive dimension, the one that Leonardo Sciascia had to

8 Tomasi di Lampedusa (1958).

9 Bufalino (1985). 
define 'sicilitudine', ${ }^{\mathbf{1 0}}$ often warped to sicilianist purpose who were certainly far from the intention of the renowned writer of Racalmuto.

But the perception of the different Sicilies is not only literary: who travel over the Island is really surprised by the strong contrasts, from Sicily of fire and 'sciare' of lava looking at themselves in a too much blue sea, to the expanses of wheat of the inland, moved in the summer by the wind like waves; from the white quarries of Siracusa to the frightening cliffs of the hinterland of Palermo; by the green forests of the Val Demone to the parched feuds of Caltanissetta. Each Sicily has generated people perceived in different ways, almost to acknowledge the existence of these internal boundaries understood even as anthropological barriers. This retiring himself inside of own land searching deep fractures is the cause and the result of atavics hatreds between towns, between villages and inwardly between quarters, to the so-called ecclesiastical competitions that impassion the people of many towns, torn apart between the observance of the cults of various patron Saints.

Events which hide social struggles, 'political', as grudges between towns of the crown and feudal villages, between towns of recent fortune and ancient metropolis decayed, resentments sometimes reached to bloody endings, as the sacking of Caltanissetta in the revolution of 1820; or symbolic, like the dispossession of Siracusa by the role of capovalle after the revolt of 1837. Law and institutions are deeply intertwined in these events, bringing to the design of judicial geography, ${ }^{11}$ which has seen between the nineteenth and the twentieth century the change of the headquarters of tribunals and courts with reasons sometimes surprising, as happened under fascism for the court of Mistretta, deleted because in the Council of Ministers «had prevailed the criteria of leaving courts in the towns of the plain in preference to those places in the mountains». Judicial districts which see also the rise of Councils of the Bar Association: as I already have had the opportunity to assert, «the historical connotations, social, economic, and even the state of the roads have formed ranks of lawyers with their own characteristics in each reality». ${ }^{12}$

10 SCIASCia (1970).

11 Cappuccio (2013).

12 Pace Gravina (2013). 
The penological science of Positivist school - and harangues of the lawyers engaged in the defence of their clients - they even drew highly imaginative genealogies of criminals, fancifully acknowledging the ancient fracture marked by the river Salso between eastern and western Sicily as a boundary between distinct criminals inclinations. So the followers of Lombroso distinguished in the first, corresponding to the portion of the Island most involved by the early Greek colonization, the dominance of the Levantine element. Few crimes of blood, prevalence of less serious offences, and that require qualities of intelligence and shrewdness: swindlers, forgers, pickpockets, treated almost with lightness and regard for the spent talent in the art of crime. ${ }^{13}$ Over the Salso river instead still operate the dark drives of the Phoenicians, Saracens and Albanians: criminals generated by these lands were perpetrators of violent crimes, heinous, vicious, with profusion of blood, which certainly denoted the fierceness of races born from the desert, whose mentality neither the slow elapsing of the centuries had failed to extirpate. Emblematic is what was written on this subject by Enrico Ferri, in his famous essay about L'Omicidio nell'antropologia criminale:

«la Sicilia offre pure un esempio evidente dell'influenza etnica sull'omicidio. Le provincie orientali di Messina, Catania e Siracusa hanno una intensità di omicidii semplici e qualificati [...] molto inferiore a quella delle provincie di Caltanissetta, Girgenti, Trapani e Palermo. Ora è noto che la Sicilia [...] presenta nelle sue coste orientali una prevalenza di elementi ellenici, dai Magno-greci in poi, che è impossibile non mettere in relazione colla minore intensità di omicidii di quel versante $[\ldots]$ ed una prevalenza invece di elementi saraceni ed albanesi nella parte meridionale e settentrionale, che certamente concorrono a determinare maggiore intensità di omicidii in quelle provincie».

Ferri quoted in support of these theories, as well as the anthropologist Giustiniano Nicolucci, even Élisée Reclus, the most famous geographer of the late nineteenth century. ${ }^{14}$

The next step was played in the perception of the Mafia: still Salso river demarcated a Sicily babba (stupid), the East (especially the province of Siracusa, including the two current of Siracusa and Ragusa), rich of towns more or less large, often demesne of the crown, from Western Sicily, rural and feudal dominated by the Mafia. Of course this to being unhooked of Eastern Sicily from the criminal shoves of the Western is not reassuring: the

13 Emblematic the case of the forger Paolo Ciulla: Attanasio (2007).

14 See e.g. Ferri (1895) 266-270. 
rhetoric of politicians and jurists reassured the public opinion, causing a serious delay in realizing of the dangers of organized crime; moreoever, this was often right in Sicily 'Babba' a free zone to go into hiding, less exposed to strict controls by the police.

In the words of the great constitutionalist Giogio Arcoleo, Sicily regains its insular intergrity in the spirit of the people:

«Nessun popolo come il siciliano, ha mostrato tanto impeto di ribellione e tanta saviezza politica. È virtù etnica che tempera effetti e difetti. Ma fulmineo lo scettro, tenace il proposito: - rispetta la gerarchia, non tollera la prepotenza: ambisce la gloria, ma affronta il sacrificio: - ama la indipendenza, ma vuole un governo: - è religioso, non clericale: pronto alla rivoluzione, vuole una costituzione megalomane, sia pure, perché l'esperienza degli orizzonti e del mare che ne circonda, impedisce le minute visioni e suscita l'istinto della grandezza e l'ideale di una patria che prevalga oltre i limiti del territorio». ${ }^{15}$

At the end of these pages reappears a Sicily by different facets: the one that still offers us the pen of Vincenzo Consolo in one of his last masterpieces, L'olivo e l'olivastro. ${ }^{\mathbf{1 6}}$ The way that the modern Ulisses faces across the Island is not at all reassuring, the interlacement of light and shadow don't vanishes in favour of the first, but reveal itself inextricable, it becomes impossible to discern the branches of two trees in an embrace that shows itself lethal. The internal boundaries are dissolved in the lucid testimony of those who observe towns and lands, as well identified in different Sicilies, where almost everywhere, inexorably, the oleaster is choking the olive tree.

\section{Bibliography}

Arcoleo, Giorgio (1932), L'anima della rivoluzione, in: Paulucci di Calboli Barone, Giacomo, Antonio Casulli (a cura di) Milano: Mondadori, II.

Aricò, Nicola (1999), Illimite Peloro, Messina: Mesogea

Attanasio, Maria (2007), Il falsario di Caltagirone. Notizie e ragguagli sul curioso caso di Paolo Ciulla, Palermo: Sellerio

Bufalino, Gesualdo (1985), Cere perse, Palermo: Sellerio

Cappuccio, Antonio (2013), La geografia giudiziaria: luoghi e interpreti della giustizia in Sicilia, in: Migliorino, Francesco, Pace Gravina, Giacomo (a cura 
di), Cultura e tecnica forense tra dimensione siciliana e vocazione europea, Bologna: Il Mulino, 411-464

Consolo, Vincenzo (1994), L'olivo e l'olivastro, Milano: Mondadori

Consolo, Vincenzo (1999), Di qua dal faro, Milano: Mondadori

Ferri, EnRICo (1895), L'omicidio nell'antropologia criminale (omicida nato e omicida pazzo), Torino: Fratelli Bocca Ed.

Kislinger, Ewald (2005), Demenna. Città e territorio, storia e archeologia, in: Nuove ricerche sul Vademone medievale, Atti del convegno S. Marco d'Alunzio, 11 settembre 2004, S. Agata di Militello: Ed. del Rotary Club, 7-35

Marrone, Antonino (2011), Circoscrizioni amministrative, compiti e reclutamento dei giustizieri siciliani dal 1282 al 1377, in: Mediterranea. Ricerche storiche $21,17-50$

Mazzarella, Salvatore (1984), Dell'isola Ferdinandea e di altre cose, Palermo: Sellerio

Mirabella, Santino (2015), L'isola passeggera, Roma: Vertigo

Pace Gravina, Giacomo (2013), Per una antropologia dell'avvocato siciliano dell'Ottocento, in: Migliorino, Francesco, Pace Gravina, Giacomo (a cura di), Cultura e tecnica forense tra dimensione siciliana e vocazione europea, Bologna: Il Mulino, 15-63

Palmieri, Niccolò (1847), Saggio storico e politico sulla Costituzione del regno di Sicilia infino al 1816, con un'appendice sulla Rivoluzione del 1820, Losanna: S. Bonamici e Compagni

Sciascia, Leonardo (1970), La corda pazza. Scrittori e cose della Sicilia, Torino: Giulio Einaudi

Tomasi di Lampedusa, Giuseppe (1958), Il Gattopardo, Milano: Feltrinelli 


\section{Contributors}

Alejandro Agüero (aleaguero@hotmail.com) is Professor of Legal History at the National University of Córdoba (Argentina), researcher of the Argentinian National Research Council (CONICET), member of the Institute for Research in Legal History (INHIDE - Buenos Aires) and member of the group HICOES (HIstoria COnstitucional de ESpaña). His research has focused on history of Spanish criminal justice in colonial context. His current research is concerned on the transition from colonial institutional order to the republican era in Argentina (19 ${ }^{\text {th }}$ century). Among his works: Castigar y perdonar cuando conviene a la República: la justicia penal de Córdoba del Tucumán, siglos 17. y 18., Madrid, 2008.

Javier Barrientos Grandon (javier.barrientos@uam.es) is Academic of the Chilean Academy of History and correspondent of the Spanish Royal Academy of History. After having taught at the Diego Portales University of Santiago (Chile), he is now researcher »Ramón y Cajal« of the Universidad Autónoma de Madrid (Spain). He has devoted his research to legal history of Hispanic America, and currently focuses its work on the history of codification. He is the author, among others books, of Historia del Derecho Indiano. Del descubrimiento colombino a la codificación (Roma, 2000), El gobierno de las Indias (Madrid, 2004), and Historia del Código de Comercio de la República de Chile (Santiago de Chile, 2015).

Laura Beck Varela (laura.beck@uam.es) is Associate Professor of Legal History at the Universidad Autónoma de Madrid (Spain). She accomplished her $\mathrm{PhD}$ studies in the European Doctoral Programme of the University of Seville (Spain) and the Goethe University of Frankfurt am Main (Germany) (2008). She is a member of the international research group HICOES (HIstoria COnstitucional de ESpaña). Her main research fields include history of private law (property rights, family law), history of legal education, book history, censorship and diffusion of legal literature between the early modern 
and the nineteenth century. Among her works: Literatura jurídica y censura: fortuna de Vinnius en Espaňa, Valencia, 2013.

Paolo Cappellini (paolo.cappellini@unifi.it) is Full Professor for Legal History, President of the School of Law, and Director of the Centro di studi per la storia del pensiero giuridico moderno at the Università di Firenze (Italy). His studies are devoted to the history of codifications, and history of juridical thought with reference to topics of private law, legal theory, criminal law. Among his works: Systema iuris, 1. Genesi del sistema e nascita della scienza delle Pandette, 2. Dal sistema alla teoria generale, Milano, 1984-85, vol. 2; Storie di concetti giuridici, Torino, 2010.

Floriana Colao (floriana.colao@unisi.it) is Full Professor of Legal History at the Faculty of Law of the Università di Siena (Italy). She is a member of the Italian Society for Legal History and of the Accademia degli Intronati in Siena. Her main research fields include history of criminal justice (XVIII-XX centuries); history of Italian university; and history of legal professions in Italy (XIX-XX centuries). Among her works: Il delitto politico tra Ottocento e Novecento. Da delitto fittizio a nemico dello Stato, Milano, 1986; Avvocati del Risorgimento nella Toscana della restaurazione, Bologna, 2006; Giustizia e politica. Il processo penale nell'Italia repubblicana, Milano, 2013.

Pietro Costa (pietro.costa@unifi.it) is Professor Emeritus at the Università di Firenze (Italy), where he taught legal history (previously at the Italian Universities of Macerata and Salerno). He was the Director of "Quaderni Fiorentini» from 2001 to 2011. Among his works: Civitas. Storia della cittadinanza in Europa, 4 vol., Roma-Bari, 1999-2001; Iurisdictio. Semantica del potere politico nella pubblicistica medievale, Milano, 2002 ${ }^{2}$; Democrazia politica e Stato costituzionale, Napoli, 2006; Il diritto di uccidere. L'enigma della pena di morte, Feltrinelli, Milano, 2010; Poucos, muitos, todos. Lições de história da democracia, Curitiba, 2012.

Marta Lorente Sariñena (marta.lorente@uam.es) is Full Professor of Legal History at the Universidad Autónoma de Madrid (Spain). Disciple of Francisco Tomás y Valiente, since its foundation she belongs to the research group HICOES (HIstoria COnstitucional de ESpaña). Her studies are devoted to the history of the Hispanic world, particularly on justice, constitutionalism 
and juridical culture. Her recent book, M. Lorente, J. M. Portillo (dirs.), El momento gaditano. La Constitución en el orbe hispánico, 1808-1826, Madrid, 2012, has been awarded the National Prize of the Spanish Parliament. Among her works La Nación y las Españas. Representación y territorio en el constitucionalismo gaditano, Madrid, 2010.

Massimo Meccarelli (massimo.meccarelli@unimc.it) is Full Professor of Legal History at the Università di Macerata (Italy) and affiliate researcher at the Max Planck Institute for European Legal History. His studies are devoted to the history of juridical thought and history of justice, on topics concerning legal theory, public law, criminal law, legal spaces. Among his works: Le Corti di cassazione nell'Italia unita. Profili sistematici e costituzionali della giurisdizione in una prospettiva comparata (1865-1923), Milano, 2005; The Assumed Space: Pre-reflective Spatiality and Doctrinal Configurations in Juridical Experience, in: Rechtsgeschichte 23 (2015), 241-252 (available at: http://dx.doi.org/10.12946/rg23/241-252).

Giacomo Pace Gravina (gpace@unime.it) is Full Professor of Legal History at the Università di Messina (Italy). His main research interests are: history of codification; legal professions; imputability of minors; law-literature; history of university. Among his works: Il discernimento dei fanciulli. Ricerche sulla imputabilità dei minori nella cultura giuridica moderna, Torino, 2000; Contrainte par corps. L'arresto personale per debiti nell'Italia liberale, Torino, 2004; Il Codice e la sciabola. La giustizia militare nella Sicilia dei Borbone tra repressione del dissenso politico ed emergenza penale (1819-1860), Acireale-Roma, 2015.

María Julia Solla Sastre (mjulia.solla@uam.es) is associate professor in legal history at the Universidad Autónoma de Madrid (Spain) and belongs to the study group HICOES (HIstoria COnstitucional de ESpaña). Her research has focused on history of Spanish judiciary. Her present research interests deal with administrative law traditions and with Spanish colonial law. Among her works, a particular stress must be laid on La discreta práctica de la disciplina. La construcción de las categorías de la responsabilidad judicial en España, 1834-1870, Madrid, 2011; also, Ultramar excepcional. La construcción de un espacio jurídico para España y sus colonias, 1837-1898, in: Rechtsgeschichte 23 (2015), 222-238 (available at: http://dx.doi.org/10.12946/rg23/222-238). 



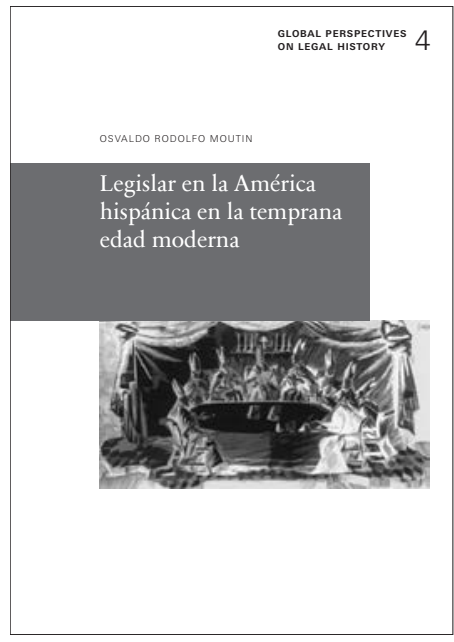

Osvaldo Rodolfo Moutin

Legislar en la América hispánica en la temprana edad moderna

Procesos y características de la producción de los Decretos del Tercer Concilio

Provincial Mexicano (1585)

Global Perspectives on Legal History 4

Frankfurt am Main: Max Planck Institute for European Legal History 2016.

X, 206 S., € 11,64 D

ISBN: 978-3-944773-03-2

Open Access Online Edition:

http://dx.doi.org/10.12946/gplh4

The decrees of the Third Mexican Provincial Council, celebrated in 1585, had an important impact on the organization of the colonial Church and SpanishAmerican society, from the time they were published in 1622 until the nineteenth century. This research focuses on how the decrees were drafted, taking into consideration the working materials used in the conciliar meeting room by the bishops and their assistants, and thus highlighting the intentions of the conciliar fathers in their new and original elaboration of both Canon Law and Derecho Indiano. 
Global Perspectives on Legal History is a book series edited and published by the Max Planck Institute for European Legal History, Frankfurt am Main, Germany. As its title suggests, the series is designed to advance the scholarly research of legal historians worldwide who seek to transcend the established boundaries of national legal scholarship that typically sets the focus on a single, dominant modus of normativity and law.

The series aims to privilege studies dedicated to reconstructing the historical evolution of normativity from a global perspective.

It includes monographs, editions of sources, and collaborative works. All titles in the series are available both as premium print-on-demand and in the open-access format.

\section{Released volumes}

4 | Osvaldo Rodolfo Moutin

Legislar en la América hispánica en la temprana edad moderna.

Procesos y características de la producción de los Decretos del Tercer Concilio

Provincial Mexicano (1585)

3 | Thomas Duve, Heikki Pihlajamäki (eds.)

New Horizons in Spanish Colonial Law.

Contributions to Transnational Early Modern Legal History

2 María Rosario Polotto, Thorsten Keiser, Thomas Duve (eds.)

Derecho privado y modernización.

América Latina y Europa en la primera mitad del siglo XX

1 | Thomas Duve (ed.)

Entanglements in Legal History: Conceptual Approaches

More information on the series and forthcoming volumes: http://global.rg.mpg.de 



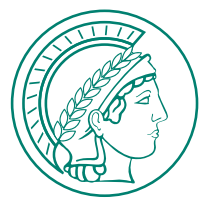

The spatiotemporal conjunction is a fundamental aspect of the juridical reflection on the historicity of law. Despite the fact that it seems to represent an issue directly connected with the question of where legal history is heading today, it has still not been the object of a focused inquiry. Against this background, the book's proposal consists in rethinking key confluences related to this problem in order to provide coordinates for a collective understanding and dialogue.

$$
\text { http://global.rg.mpg.de }
$$

ISBN 978-3-944773-05-6 